Prepared in cooperation with the U.S. Army Fort Irwin National Training Center

\title{
Evaluation of Land Subsidence and Ground Failures at Bicycle Basin, Fort Irwin National Training Center, California, 1992-2017
}

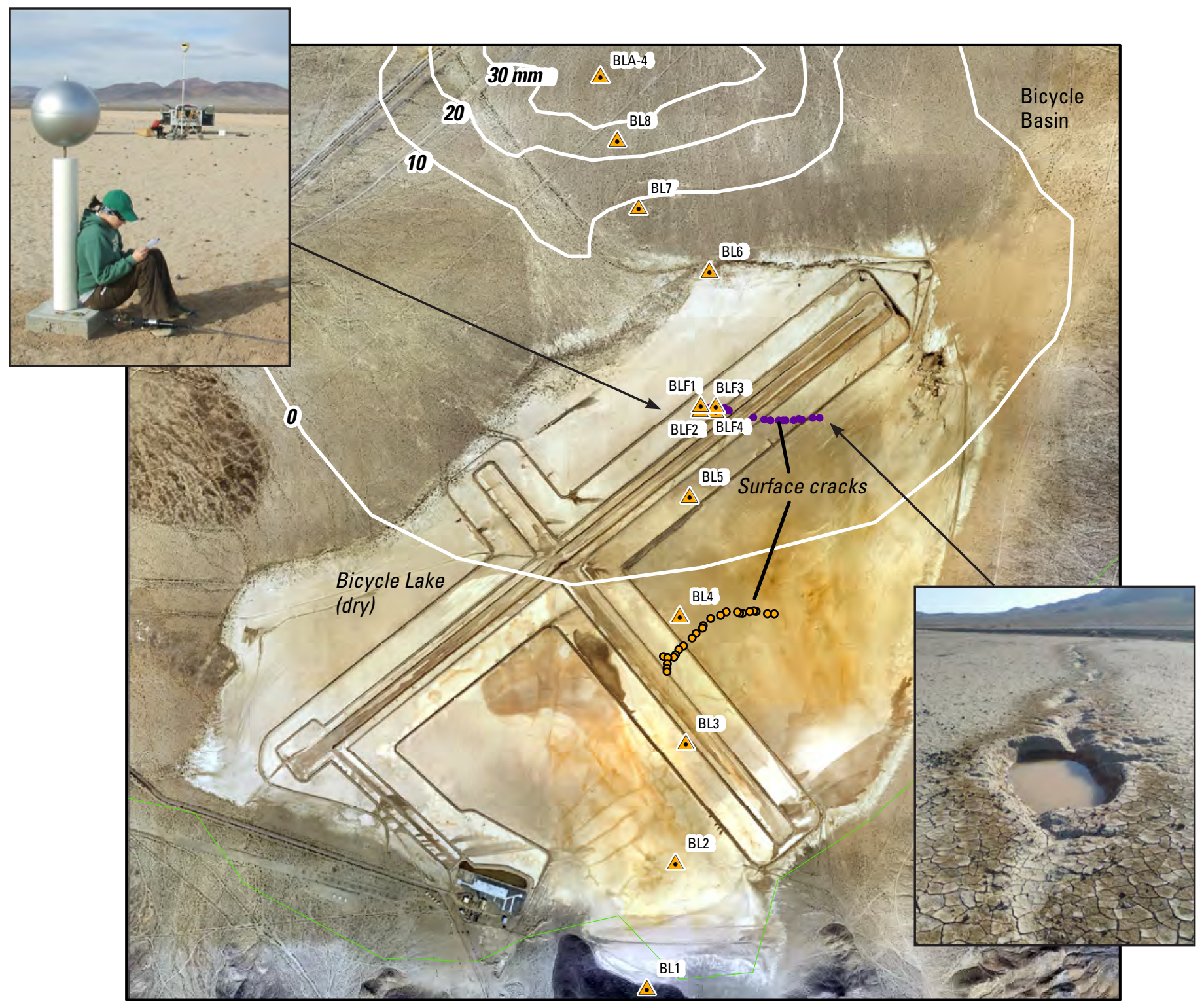

Scientific Investigations Report 2019-5015 


\section{Cover photographs}

Front cover: Aerial view of the Bicycle Basin and Bicycle Lake (dry) playa (image from Fort Irwin National Training Center, 2014) showing subsidence contours for November 2003-January 2005, and photographs of crack that formed in 2005-06, and lidar monument. Photographs taken in 2011 and 2009, respectively, by J.N. Densmore, U.S. Geological Survey.

Back cover: Photographs of 2005-06 crack at the surface and in trench dug across the crack. Photographs taken during April 2007, provided by Justine Dishart, U.S. Army. 


\section{Evaluation of Land Subsidence and Ground Failures at Bicycle Basin, Fort Irwin National Training Center, California, 1992-2017}

By Jill N. Densmore, Kevin M. Ellett, Michelle Sneed, Justin T. Brandt, James F. Howle, Andrew Y. Morita, Rodrigo Borela, Antonio Bobet, and Drew C. Thayer

Prepared in cooperation with the U.S. Army Fort Irwin National Training Center

Scientific Investigations Report 2019-5015 


\title{
U.S. Department of the Interior \\ DAVID BERNHARDT, Secretary
}

\author{
U.S. Geological Survey \\ James F. Reilly II, Director
}

U.S. Geological Survey, Reston, Virginia: 2019

For more information on the USGS - the Federal source for science about the Earth, its natural and living resources, natural hazards, and the environment-visit https://www.usgs.gov or call 1-888-ASK-USGS.

For an overview of USGS information products, including maps, imagery, and publications,

visit https://store.usgs.gov.

Any use of trade, firm, or product names is for descriptive purposes only and does not imply endorsement by the U.S. Government.

Although this information product, for the most part, is in the public domain, it also may contain copyrighted materials as noted in the text. Permission to reproduce copyrighted items must be secured from the copyright owner.

Suggested citation:

Densmore, J.N., Ellett, K.M., Sneed, M., Brandt, J.T., Howle, J.F., Morita, A.Y., Borela, R., Bobet, A., and Thayer, D.C., 2019, Evaluation of land subsidence and ground failures at Bicycle Basin, Fort Irwin National Training Center, California, 1992-2017: U.S. Geological Survey Scientific Investigations Report 2019-5015, 93 p., https://doi.org/10.3133/sir20195015. 


\section{Acknowledgments}

The authors thank the following personnel at Fort Irwin National Training Center, Justine Dishart, Muhammed Bari, and Chris Woodruff for providing funding and guidance on this work; Miles Hubbard, Gerald Espinosa, and Elisa Sanchez for assisting with data

collection; and Jennifer Fitzpatrick and Martin Fish for access to Bicycle Lake Airfield. 


\section{Contents}

Abstract

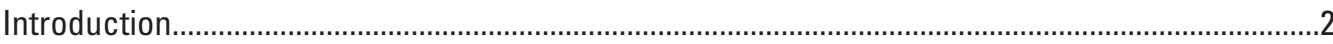

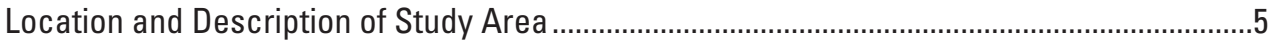

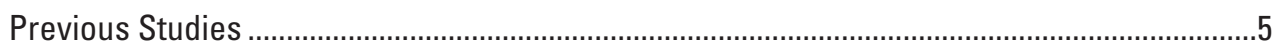

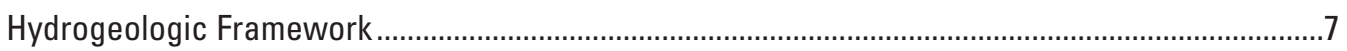

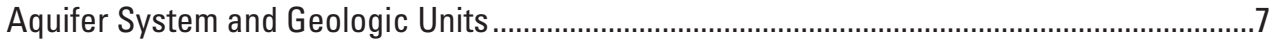

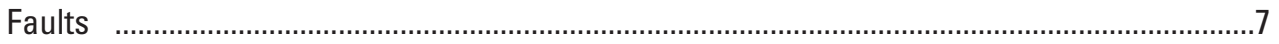

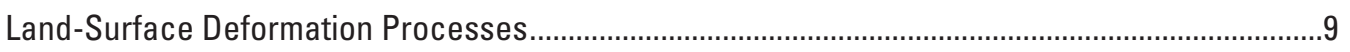

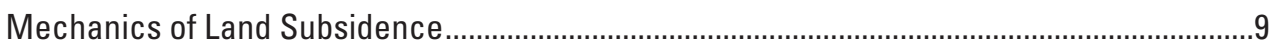

Surface Cracking - Earth Fissures, Surface Faults, and Giant Desiccation Cracks of

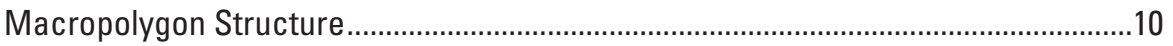

Measurements and Methods.................................................................................................13

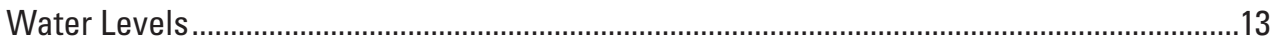

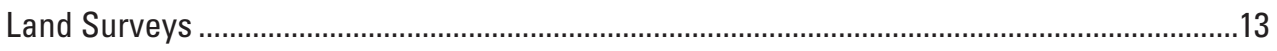

Monument Network Establishment ............................................................................13

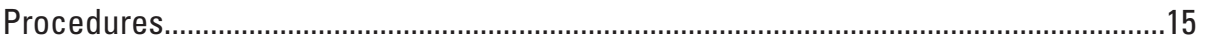

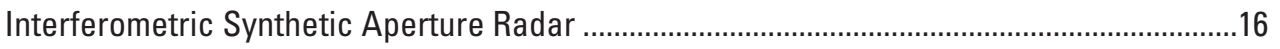

Surface Geophysical Surveys ......................................................................................17

Numerical Experiments of Soil Mechanics and the Cracking Process..................................23

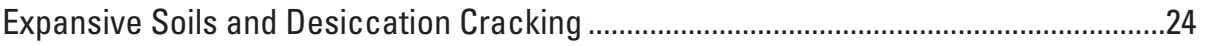

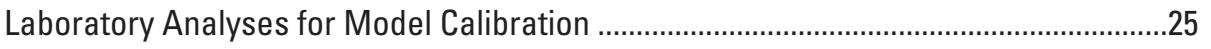

Discrete Element Method and Model Calibration ..............................................................26

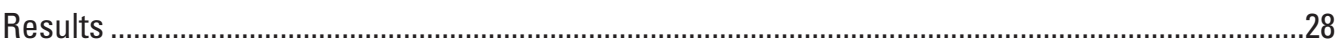

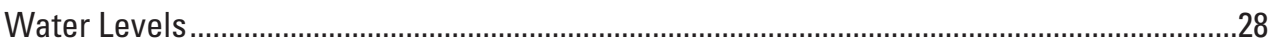

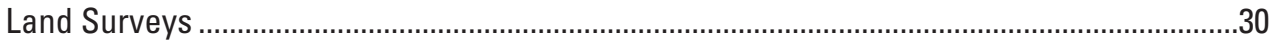

Vertical Land-Surface Change Along Leveling Transect................................................31

Ground Movement Across the 2005-06 Main-Runway Crack ........................................33

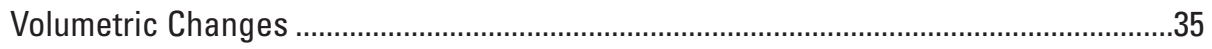

Interferometric Synthetic Aperture Radar ........................................................................42

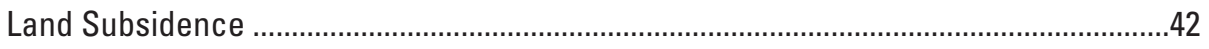

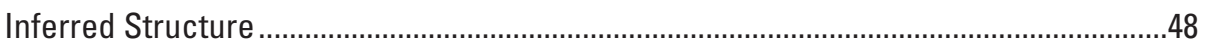

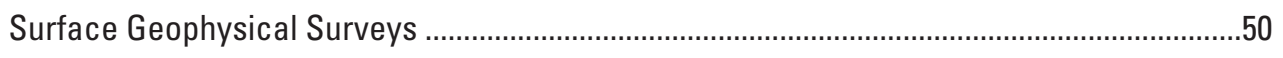

Subsurface Imaging Near the Main-Runway Crack......................................................50

Electromagnetic Induction Mapping for Ground Failures Assessment and

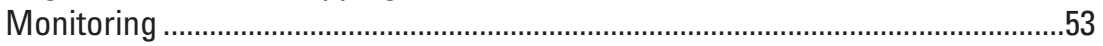

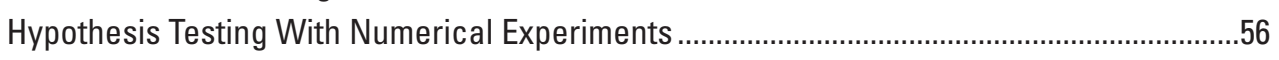

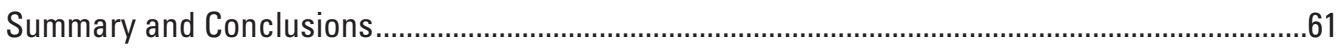

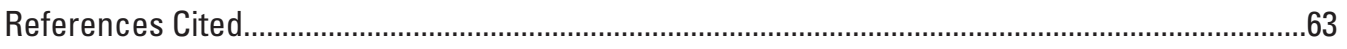

Appendix 1. Summary of Construction Data for Production and Monitoring Wells in the Bicycle Basin, Fort Irwin National Training Center, California ............................................68

Appendix 2. Water-Level Data for Selected Wells in Bicycle Basin, Fort Irwin National Training Center, California, 1955-2017 .........................................................................70 


\section{Figures}

1. Map showing the location of study area, Fort Irwin National Training Center, California .....

2. Photographs of Bicycle Lake playa, Fort Irwin National Training Center, California, showing the location of macropolygons and large surface cracks that formed in 2006 and 2013.......

3. Map showing the generalized surficial geology, major faults, location of groundwater monitoring sites and production wells, and geologic section lines in Bicycle Basin, Fort Irwin National Training Center, California.

4. Generalized geologic sections across the Bicycle Basin, Fort Irwin National Training Center, California.....

5. Cross-sections illustrating hypotheses for the formation of Earth fissures and surface faults driven by differential compaction associated with water-table declines from groundwater pumping

6. Cross-sections illustrating previous hypotheses for the formation of macropolygon-patterned ground structure found on dry playas throughout the southwest United States ....

7. Map showing monument network for geodetic control in Bicycle Basin, Fort Irwin National Training Center, California.

8. Photographs of geophysical survey data-collection surveys.

9. Graph showing results from the test of repeatability along a 3-kilometer survey line of the main runway, December 2014, Fort Irwin National Training Center, California, indicating average variability for repeat survey lines is around 7 millisiemens per meter.

10. Images showing results of playa soil desiccation according to the gravimetric water content, in percent, for discrete element method model parameterization and calibration

11. Illustration showing numerical experiment to evaluate the conventional hypothesis of macropolygon formation as giant desiccation cracks from a declining water table at depth.

12. Illustration showing the numerical experiment to evaluate the potential role of regional tectonic stress in propagating extensional cracks upward to the land surface in conjunction with the desiccation process.

13. Hydrographs showing water-level changes in selected wells during 1955-2017 in Bicycle Basin, Fort Irwin National Training Center, California

14. Map showing water-table contours approximated from water levels measured during 2017 in Bicycle Basin, Fort Irwin National Training Center, California

15. Hydrographs showing continuous water-level changes, Bicycle Basin, Fort Irwin National Training Center, California.

16. Graph showing vertical change along transect for repeat leveling surveys relative to 2009 baseline survey in Bicycle Basin, Fort Irwin National Training Center, California

17. Graph showing change in distance between monuments in Bicycle Basin, Fort Irwin National Training Center, California.

18. Images showing vertical land-surface change for 2005-06 main-runway crack, Fort Irwin National Training Center, California.

19. Time-series shaded-relief images for 2005-06 main-runway crack from January 2009 through December 2016, Fort Irwin National Training Center, California 
20. Images showing progression of volumetric calculation along the main-runway crack on Bicycle Lake playa, Fort Irwin National Training Center, California..

21. Interferogram showing subsidence for Bicycle Basin, Fort Irwin National Training Center, California .

22. Graphs showing relationship between water levels near the area of maximum subsidence and monthly pumpage for wells in Bicycle Basin, Fort Irwin National Training Center, California .

23. Graph showing interferogram-derived subsidence time-series data for five selected locations, 1992 to 2014, Bicycle Basin, Fort Irwin National Training Center, California

24. Illustration showing electrical resistivity tomography survey results for the area around the main-runway crack, by year, and electrode spacing, Bicycle Basin, Fort Irwin National Training Center, California.

25. Map showing apparent electrical conductivity across the Bicycle Lake airstrip region, with known ground failures of two surface cracks that have formed since 2005 outlined in black, based on results from electromagnetic induction surveys in Bicycle Basin, Fort Irwin National Training Center, California, at 18 kilohertz operating frequency.

26. Graph showing electromagnetic induction surveys for the main runway from 2008, 2014, and 2015, Bicycle Basin, Fort Irwin National Training Center, California ......56

27. Difference map showing electromagnetic induction time-lapse surveys of Bicycle Basin, Fort Irwin National Training Center, California, for 2014-15..

28. Maps showing electromagnetic induction time-lapse surveys in Bicycle Basin, Fort Irwin National Training Center, California, indicate the main-runway crack was stable during the 2014-15 period.

29. Images showing discrete element method model results for particle-stress accumulation from simulating shallow water-table drawdown

30. Images showing discrete Element Method model results for particle-stress accumulation from simulating deep water-table drawdown.

31. Images showing discrete Element Method model results for crack-propagation simulation indicates cracking induced from desiccation volumetric shrinkage in the capillary-fringe zone above a declining water table is unable to propagate upward to the shallow, overlying soil.

32. Images showing discrete element method model results for crack formation from the desiccation process alone compared to the combined processes of desiccation and tensile strain arising from a large, regional tectonic-stress field

\section{Tables}

1. Acquisition dates of synthetic aperture radar data, interferogram timelines, and subsidence magnitudes and rates for 103 interferograms analyzed for Bicycle Basin, Fort Irwin National Training Center, California, 1992-2015.

2. Summary of the change in volume of the crack calculated between sequential surveys on Bicycle Lake playa, Fort Irwin National Training Center, California 


\section{Conversion Factors}

International System of Units to U.S. customary units

\begin{tabular}{lcl}
\hline \multicolumn{1}{c}{ Multiply } & By & \multicolumn{1}{c}{ To obtain } \\
\hline centimeter $(\mathrm{cm})$ & Length & \\
millimeter $(\mathrm{mm})$ & 0.3937 & inch (in.) \\
meter $(\mathrm{m})$ & 0.03937 & inch (in.) \\
kilometer $(\mathrm{km})$ & 3.281 & foot $(\mathrm{ft})$ \\
\hline & 0.6214 & mile $(\mathrm{mi})$ \\
\hline square meter $\left(\mathrm{m}^{2}\right)$ & Area & \\
square meter $\left(\mathrm{m}^{2}\right)$ & 0.0002471 & acre \\
square kilometer $\left(\mathrm{km}^{2}\right)$ & 10.76 & square foot $\left(\mathrm{ft}^{2}\right)$ \\
\hline & 0.3861 & square mile $\left(\mathrm{mi}^{2}\right)$ \\
\hline liter $(\mathrm{L})$ & Volume & \\
million liters & 0.2642 & gallon (gal) \\
cubic meter $\left(\mathrm{m}^{3}\right)$ & $264,172.052$ & million gallons $(\mathrm{Mgal})$ \\
cubic meter $\left(\mathrm{m}^{3}\right)$ & 35.31 & cubic foot $\left(\mathrm{ft}^{3}\right)$ \\
\hline
\end{tabular}

Temperature in degrees Celsius $\left({ }^{\circ} \mathrm{C}\right)$ may be converted to degrees Fahrenheit $\left({ }^{\circ} \mathrm{F}\right)$ as

$$
{ }^{\circ} \mathrm{F}=\left(1.8 \times{ }^{\circ} \mathrm{C}\right)+32 .
$$

Temperature in degrees Fahrenheit $\left({ }^{\circ} \mathrm{F}\right)$ may be converted to degrees Celsius $\left({ }^{\circ} \mathrm{C}\right)$ as

$$
{ }^{\circ} \mathrm{C}=\left({ }^{\circ} \mathrm{F}-32\right) / 1.8 \text {. }
$$

\section{Datum}

Vertical coordinate information is referenced to the North American Vertical Datum of 1988 (NAVD 88).

Horizontal coordinate information is referenced to the North American Datum of 1983 (NAD 83).

Altitude, as used in this report, refers to distance above the vertical datum.

\section{Abbreviations}

$\begin{array}{ll}\text { 2-D } & \text { two-dimensional } \\ 3-D & \text { three-dimensional } \\ \text { DEM } & \text { digital elevation model } \\ \text { ECa } & \text { apparent electrical conductivity } \\ \text { EDM } & \text { electronic distance measurement } \\ \text { EMI } & \text { electromagnetic induction } \\ \text { ERS-1 } & \text { European Earth Remote Sensing I } \\ \text { ERS-2 } & \text { European Earth Remote Sensing II }\end{array}$




$\begin{array}{ll}\text { ERT } & \text { electrical resistivity tomography } \\ \text { FEM } & \text { finite-element method } \\ \text { InSAR } & \text { interferometric synthetic aperture radar } \\ \text { Khz } & \text { kilohertz } \\ \text { lidar } & \text { light detection and ranging } \\ \text { mS/m } & \text { millisiemens per meter } \\ \text { NTC } & \text { National Training Center } \\ \text { PVC } & \text { polyvinyl chloride } \\ \text { SAR } & \text { synthetic aperture radar } \\ \text { USGS } & \text { U.S. Geological Survey }\end{array}$

\section{Well-Numbering System}

Wells are assigned a state well number (station name) by the California Department of Water Resources according to the location in the rectangular township and range grid system for the subdivision of public lands. Station names consist of the township number, north or south; the range number, east or west; and the section number. Each section is divided into sixteen 40 -acre tracts lettered consecutively (except " $I$ " and " 0 "), beginning with " $A$ " in the northeast corner of the section and progressing in a sinusoidal manner to " $\mathrm{R}$ " in the southeast corner. Within the 40-acre tract, numbers are assigned sequentially in the order the wells are inventoried. The next letter within the station name refers to the base line and meridian. California has three base lines and meridians-Humboldt (H), Mount Diablo (M), and San Bernardino (S). Wells in the study area are referenced to the San Bernardino and Mount Diablo base line and meridian (S and $\mathrm{M}$ ). Well numbers consist of 15 characters and follow the format 012N003E01M001S. In this report, wells are abbreviated and written as 12N/03E-01M1S. Wells are abbreviated in figures by their section number, tract letter, and sequence number (for example, 1M1). In addition to a station name assigned by the California Department of Water Resources, wells were assigned a common name derived from the basin in which they were installed and a sequence number. Wells were also assigned a 15-digit site identification number in the U.S. Geological Survey National Water Information System database.

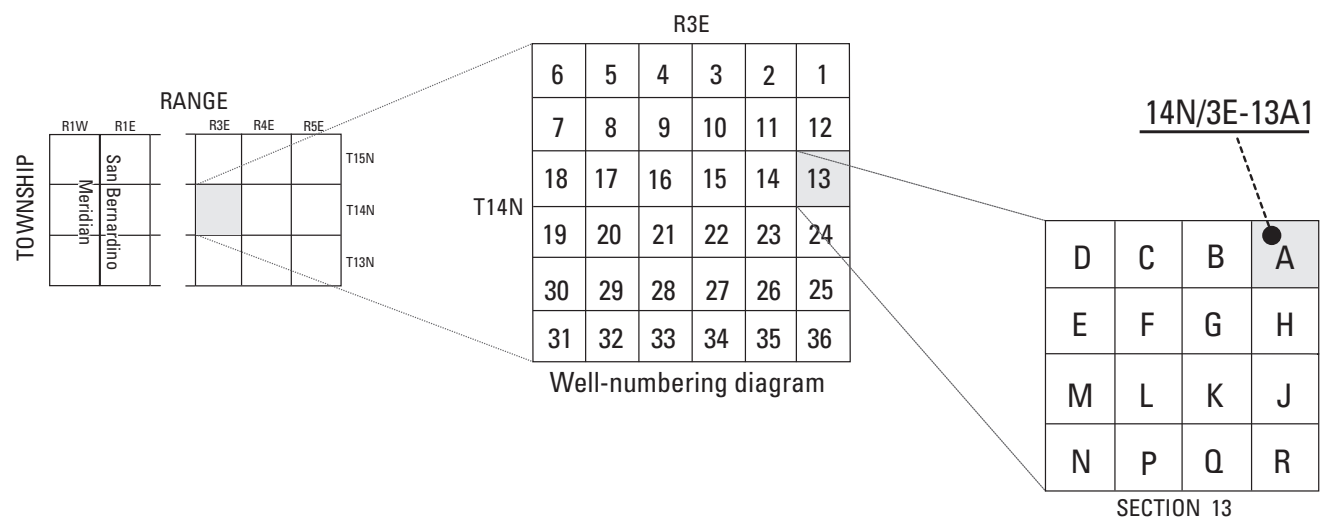




\title{
Evaluation of Land Subsidence and Ground Failures at Bicycle Basin, Fort Irwin National Training Center, California, 1992-2017
}

\author{
By Jill N. Densmore, Kevin M. Ellett, Michelle Sneed, Justin T. Brandt, James F. Howle, Andrew Y. Morita, \\ Rodrigo Borela, Antonio Bobet, and Drew C. Thayer
}

\section{Abstract}

Groundwater has been pumped in the Bicycle Basin at Fort Irwin National Training Center since the 1960s, and the amount pumped has generally increased since the 1990s. After a large crack (approximately 0.5-kilometer long) formed at the surface of Bicycle Lake playa during 2005-06 in the area used as an aircraft runway, a monitoring study was initiated by the U.S. Geological Survey, in cooperation with the U.S. Army Fort Irwin National Training Center, to help determine the cause. The extent and effect of groundwaterlevel declines and land-surface deformation in Bicycle Basin were evaluated using a number of approaches, including water-level measurements from December 2007 to June 2017, land surveys across the playa area, interferometric synthetic aperture radar (InSAR) analyses, geophysical surveys of the playa area, and numerical experiments to test hypotheses about soil mechanical processes. A specific objective of this study was to evaluate the recent development of ground failures in the form of large, surface cracks that pose a hazard to aircraft operations on the Bicycle Lake playa airstrip.

Another large crack and set of interconnected cracks formed at the surface of Bicycle Lake playa in 2013 following a period of inundation similar to that of 2005-06; however, the 2013 cracks formed a network rather than a single feature. Groundwater pumping resulted in more than 27 meters (90 feet) of water-level declines in wells north of the Bicycle Lake playa from 1990 to 2017, and InSAR interferograms indicated more than 400 millimeters (16 inches) of subsidence during 1993-2015. Subsidence rates calculated from InSAR interferograms were variable, temporally and spatially. Results of leveling surveys indicated differential subsidence between 2009 and 2016 along a transect across the playa to the area of maximum subsidence; there was less subsidence south of the 2005-06 crack than north of it. The steepest subsidence gradient for this time was in the area of maximum subsidence. Repeat tape-extensometer measurements from April 2009 to November 2017 across the main-runway crack indicated slight opening along part of the crack. A baseline lidar (light detection and ranging) survey of the main-runway crack was done in January 2009 to track the development of this feature. During the 95-months from January 2009 to December 2016, 0.5 meters (19.7 inches) of subsidence was observed next to the subsidence pit in the crack along the western end of the scanned area, presumably due to erosion and backfilling when the lake was inundated. Standing water in the crack along the eastern end of the scanned area prevented determining change for that part of the crack. Time-series, shaded-relief images show the progression of change in the crack from January 2009 through December 2016, with the crack closing and opening, depending on the time of most recent inundation relative to when the scans were taken. Volumetric changes in the size of the crack, calculated between the sequential lidar surveys, show that from 2009 to 2016, the cumulative volumetric change was 1.5 cubic meters ( 54 cubic feet). This volume is a minimum estimate because the crack volume below the water surface in the pits was not included in the calculations. Subsurface imaging of the 2005-06 main-runway crack by a series of electrical resistivity tomography surveys in 2008 and 2017 indicated that the crack could extend 5 meters (16 feet) in the subsurface.

Electromagnetic induction surveys in 2008, 2014, and 2015 evaluated the technique for ground-failure monitoring and showed that the technique was effective at revealing anomalies correlated with the features of concern in the playa area, such as the 2005-06 crack, numerous "healed" macropolygon features, and scattered sink-like depressions.

Results from numerical experiments simulating watertable decline at depth indicated that the material deposits in the desaturating capillary fringe zone might not be able to transmit large enough stresses up through the overlying soil to cause cracks at the land surface. Results from simulations of desiccation in the presence of a regional tectonically induced stress field, however, tended to support the hypothesis that the combined processes could control the formation of giant desiccation macropolygons in certain areas, such as Bicycle Lake playa. 


\section{Introduction}

The U.S. Army's Fort Irwin National Training Center (NTC), in the Mojave Desert, southern California, obtains potable water from groundwater aquifers in the Irwin, Bicycle, and Langford Basins (fig. 1). After a large crack formed in 2005-06 on Bicycle Lake playa, which is used as an aircraft runway to transport troops and supplies, a monitoring study was initiated by the U.S. Geological Survey (USGS) to help determine the cause. Groundwater has been pumped in the Bicycle Basin since the 1960s and the amount pumped has increased since the 1990s with the expansion of NTC activities and a reduction of pumping in the neighboring Irwin Basin in response to water-quality concerns related to wastewater disposal practices (Densmore and Londquist, 1997). Increased pumping in Bicycle Basin has resulted in more than 27 meters ( $\mathrm{m}$; $90 \mathrm{feet}, \mathrm{ft})$ of water-level decline in wells north of the Bicycle Lake playa from 1990 to 2017. Interferometric synthetic aperture radar (InSAR) interferograms show more than 400 millimeters (mm; 16 inches, in.) of subsidence north of Bicycle Lake playa during 1993-2015.

Bicycle Lake playa is predominantly dry (fig. 2). Landsat imagery was reviewed for this study and shows that Bicycle Lake playa only has rare periods of flooded, lake-like conditions persisting beyond a month. The typically hard crust of the playa surface allows it to be used as an aircraft runway to transport troops and supplies to the NTC. Concern over ground failures at the playa airfield, and the potential effect on aircraft operations, began in 2005-06, following a long period of lake-like conditions (roughly 140 days). After the lake evaporated and the playa sediments dried, a large crack appeared on the northeastern part of the playa and extended across the main runway of the airstrip (figs. $2 A, B$ ). This large, single crack approximately $500 \mathrm{~m}(1,640 \mathrm{ft})$ long was hypothesized to be an Earth fissure. Earth fissures are found in semi-arid to arid basins, where such tensile failures can form as a result of differential compaction in the subsurface caused by aquifer depletion (Burbey, 2010; Holzer, 2010).

Numerous sink-like depressions up to $3 \mathrm{~m}$ (10 ft) long and $1 \mathrm{~m}(3.3 \mathrm{ft})$ deep were also observed at the playa surface at this time. In some cases, these depression features coalesced in a macropolygon morphology indicative of giant desiccation cracks. Aerial photography showed that macropolygon structure (hundreds of meters in diameter), associated with giant desiccation cracks, is a persistent feature of Bicycle Lake playa, primarily in the southern part of the playa (fig. 2), and is reported to date back to at least the 1940s (Neal and others, 1968).
Following another period of lake-like conditions in 2013, a second large crack appeared at the playa surface, crossing the short northwest-southeast trending runway that is perpendicular to the main runway (fig. 2C). The general shape and depth of this large crack was very similar to the 2005-06 crack, with the notable exception of clear bifurcation into a set of interconnected cracks in what appears to be macropolygonal morphology. The 2013 crack is also somewhat perpendicular to the nearly east-west trending 2005-06 crack, and it evolved roughly a kilometer away from the 2005-06 crack in an area of the playa that has a notable macropolygon structure on its surface.

The U.S. Geological Survey completed a study, in cooperation with the U.S. Army Fort Irwin National Training Center, to address three objectives: (1) to assess the extent of land-surface deformation in Bicycle Basin and ground failures at Bicycle Lake playa, (2) to evaluate the potential mechanisms driving ground failures here and in similar locations, and (3) to provide a method for longterm monitoring and assessment of such failures. The clear presence of numerous macropolygon features on Bicycle Lake playa, both in a relict or "healed" state, as well as in an apparently active phase, complicate the interpretation of which physical processes are likely driving the development of these cracks at the playa surface. This study is intended to help the NTC develop an effective strategy to evaluate the long-term viability of the Bicycle Lake playa for aircraft operations.

This work was done in conjunction with a basin-wide study that describes the geohydrologic and geochemical framework of the Bicycle Basin (Densmore and others, 2018). The companion study included a groundwater-flow model of the Bicycle Basin region to help evaluate the longterm availability of groundwater for the NTC. The findings presented in this report were incorporated in the calibration process for the subsidence component of the groundwater-flow model. The calibrated groundwater-flow model was then used to help evaluate the relation between groundwater pumping and land-surface deformation, including subsidence and the formation of ground failures at Bicycle Lake playa. Work in the companion report also included documentation of a gravity survey to estimate the depth of bedrock (or basement complex) in Bicycle Basin (Robert Jachens, U.S. Geological Survey, written commun., 2007), seismic-refraction surveys at three locations to determine the depth of Quaternary and Tertiary sediment layers in the basin (David Berger, U.S. Geological Survey, written commun., 1996), and installation of six multiple-well monitoring sites during 1993-2011 to provide depth-dependent geohydrologic and geochemical data. Together, the reports provide a comprehensive understanding of the Bicycle Basin region. 


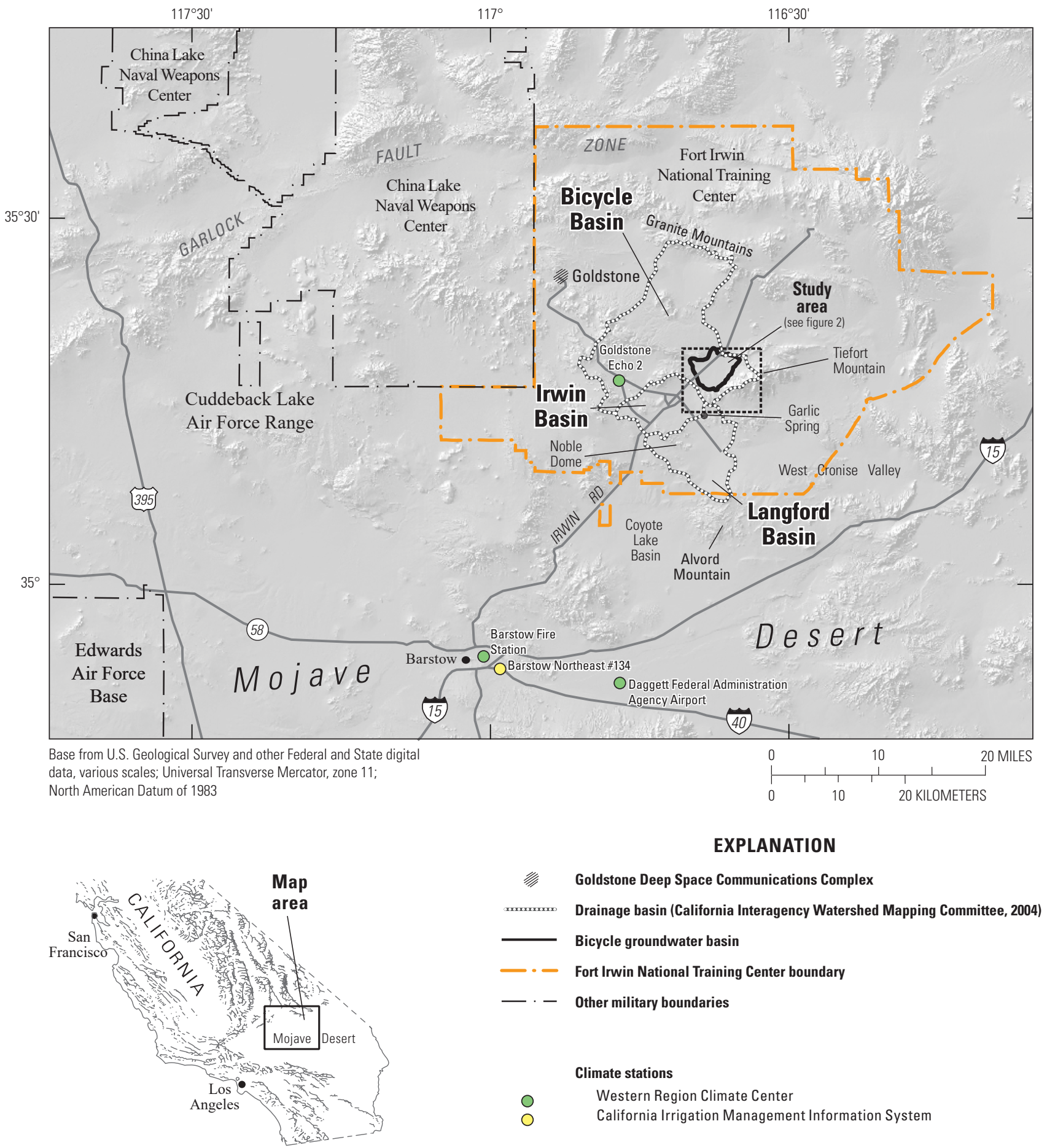

Figure 1. Location of study area, Fort Irwin National Training Center, California. 


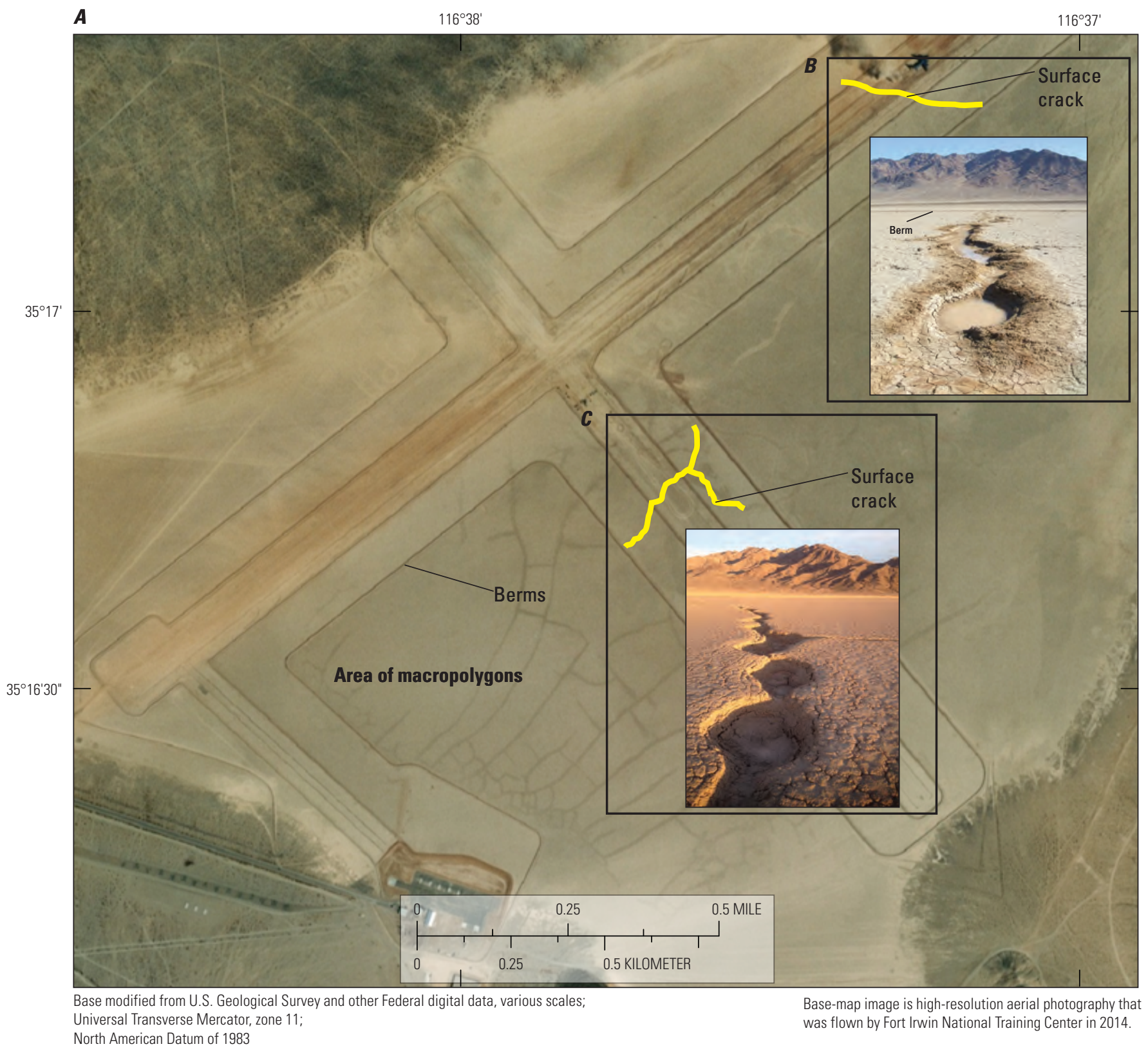

Figure 2. Bicycle Lake playa, Fort Irwin National Training Center, California, showing the location of macropolygons and large surface cracks that formed in 2006 and 2013. 


\section{Location and Description of Study Area}

Fort Irwin NTC is about 210 kilometers (km; 130 miles, mi) northeast of Los Angeles in the Mojave Desert region of southern California (fig. 1) and about $56 \mathrm{~km}$ (35 mi) northeast of Barstow, California. The NTC covers an area of about 3,048 square kilometers $\left(\mathrm{km}^{2} ; 1,177\right.$ square miles, $\left.\mathrm{mi}^{2}\right)$ that contains several surface-water drainage basins, including Irwin, Bicycle, and Langford Basins.

Bicycle Groundwater Basin (fig. 1), referred to as Bicycle Basin in this report, lies in the southeastern part of the much larger Bicycle Valley drainage basin (about $360 \mathrm{~km}^{2}$ or $140 \mathrm{mi}^{2}$ ); the Bicycle Basin covers an area of about $27 \mathrm{~km}^{2}$ $\left(10.5 \mathrm{mi}^{2}\right)$. Bicycle Basin, typical of desert basins in the Mojave Desert, is a closed basin with a relatively flat floor surrounded by generally rugged mountains or low-lying hills. Bicycle Valley drainage basin is bounded to the north by the Granite Mountains, to the east by Tiefort Mountain, to the south by low-lying hills that separate Bicycle Basin from Irwin Basin, and to the west by low-lying hills that separate Bicycle Basin from the highlands near Goldstone (fig. 1). Bicycle Lake, a usually dry playa lake, lies in the southern part of Bicycle Basin (fig. 3). The floor of Bicycle Basin ranges in altitude from about $716 \mathrm{~m}(2,350 \mathrm{ft})$ above North American Vertical Datum of 1988 (NAVD 88) on the playa to about $792 \mathrm{~m}(2,600 \mathrm{ft})$ above NAVD88 at the base of Tiefort Mountain. No perennial streams are in the basin, but numerous washes have streamflow for several days after large storms and can flood the playa in the lowest part of the basin. Little vegetation grows on or at the margins of Bicycle Lake playa; a small vegetated area that might be supported by a localized, shallow perched zone, or a buried well described in Mendenhall (1909, p. 54), is on the northeastern part of the playa.

The climate of Bicycle Basin and the NTC, typical of the Mojave Desert region, is characterized by low precipitation, hot summers, and cool winters. Only sporadic meteorological records were available for the Bicycle Basin area, primarily for the years of 2003-08. Long-term meteorological records (1950 onward) were available for the nearby Goldstone
Echo 2 station about $18 \mathrm{~km}$ (11 mi) west of the basin (fig. 1), indicating that mean annual precipitation was about $170 \mathrm{~mm}$ (7 in.) and ranged between $51 \mathrm{~mm}$ ( 2 in.) and $305 \mathrm{~mm}$ (12 in.; National Oceanic and Atmospheric Administration, 1994, 2010, 2014, http://www.ncdc.noaa.gov/, accessed September 2, 2015). Most precipitation falls during the winter, but some additional precipitation from isolated thunderstorms falls during the summer.

The average annual temperature at the nearby Barstow station (fig. 1) was about 18 degrees Celsius $\left({ }^{\circ} \mathrm{C}\right.$; 64 degrees Fahrenheit, ${ }^{\circ} \mathrm{F}$ ) for the period of record spanning 1940 2013 and ranged between $-16\left(3^{\circ} \mathrm{F}\right)$ and $49^{\circ} \mathrm{C}\left(121^{\circ} \mathrm{F}\right.$; EarthInfo, Inc., 1995, 2000; California Irrigation Management Information System, accessed on September 2, 2015, at URL http://wwwcimis.water.ca.gov/WSNReportCriteria.aspx\#). The average annual potential evapotranspiration greatly exceeded precipitation in the region. Potential evapotranspiration was reported to be 3,760 mm (148 in.) annually in Death Valley, about $56 \mathrm{~km}(35 \mathrm{mi})$ northeast of the basin (Densmore and Londquist, 1997), and about 1,930 mm (76 in.) at Newberry Springs, about $40 \mathrm{~km}(25 \mathrm{mi})$ to the south of the basin (David Inouye, California Department of Water Resources, written commun., 1996). More recent studies by Bedinger and Harrill (2012) indicated that potential evapotranspiration was much less that the $3,760 \mathrm{~mm}$ (148 in.) reported in Death Valley. As of 2018, there were no evapotranspiration estimates for Fort Irwin.

\section{Previous Studies}

Previous studies of the Bicycle Basin include those by the USGS and several consulting and engineering companies. Kunkel and Riley (1959) reported on a hydrogeological reconnaissance of the basin, and Yount and others (1994) published a report on detailed geologic mapping. Groundwater-availability studies were published by C.F. Hostrup and Associates (1955), James M. Montgomery and Associates (1981), Wilson F. So and Associates (1989), and most recently, Densmore and others (2018). 

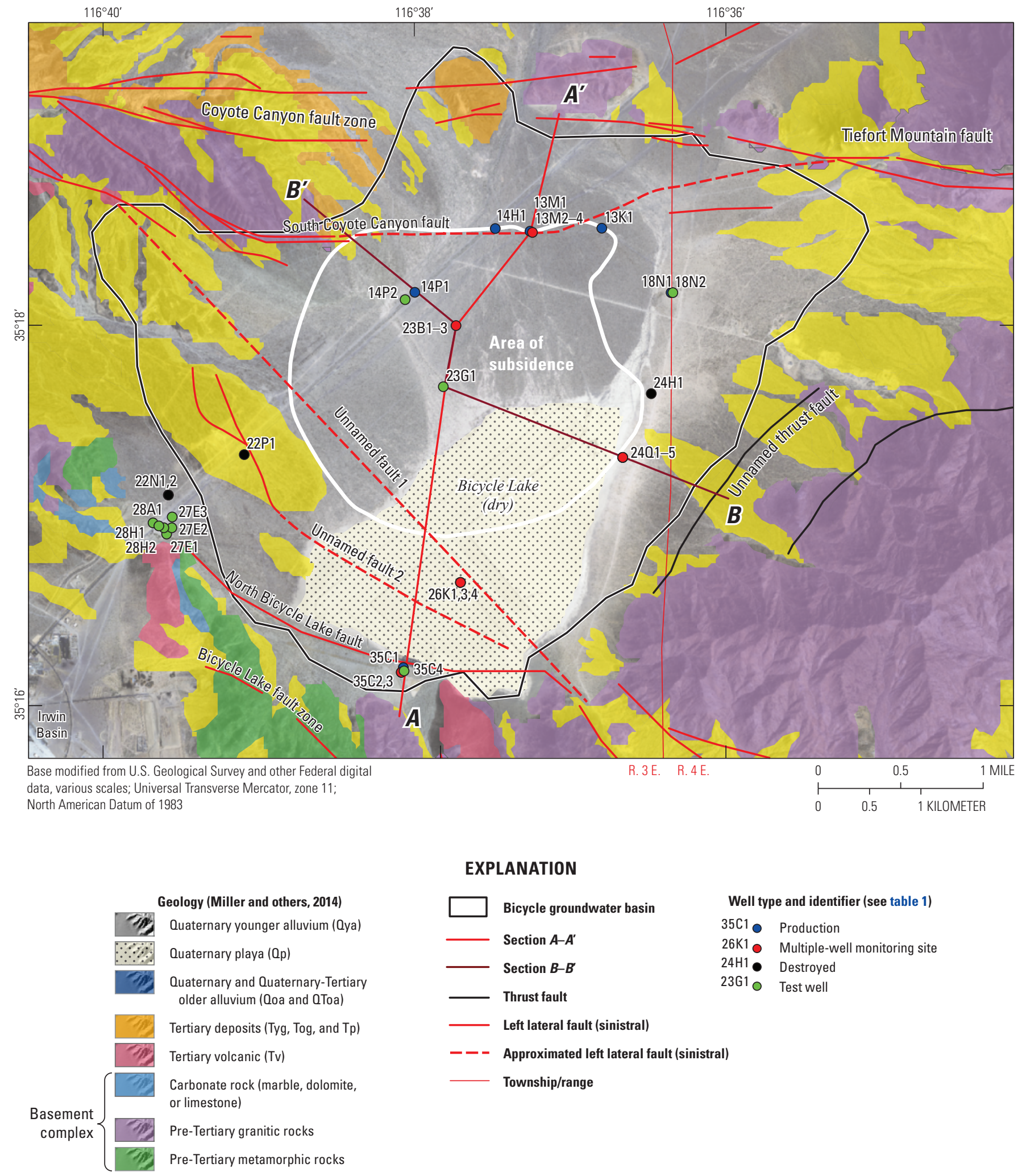

Figure 3. Generalized surficial geology, major faults, location of groundwater monitoring sites and production wells, and geologic section lines in Bicycle Basin, Fort Irwin National Training Center, California. 


\section{Hydrogeologic Framework}

The Bicycle Basin aquifer system was defined from previous studies (Densmore and others, 2018). Information about the aquifer system was supplemented by geophysical investigations completed during this study, and geohydrologic data collected from existing and newly installed wells in the basin (fig. 3). Figure 3 shows the geologic units that form the hydrogeologic framework and make up the aquifer system.

\section{Aquifer System and Geologic Units}

The Bicycle Basin aquifer system consists of an upper aquifer and lower aquifer. The upper aquifer is composed of the saturated part of the Quaternary younger alluvium and playa deposits ( $Q y a$ and $Q p$ ), the Quaternary older alluvium (Qoa), and Quaternary-Tertiary older alluvium and lacustrine deposits ( $Q T o a$ and $Q T o l)$ and generally is unconfined (fig. 4). The lower aquifer is composed of Tertiary younger and older sedimentary deposits (Tyg and $T o g$ ) and generally is confined or partly confined. The base of the aquifer system is considered to be the top of the basement complex $(B C)$, which is assumed to be impermeable. It is believed that the deepest part of the Tertiary older sedimentary deposits also do not produce much water.

The basement complex $(B c)$ consists of pre-Tertiary gneiss, metasedimentary rocks and granitics, as well as metavolcanic and carbonate rocks (Yount and others, 1994; Schermer and others, 1996; Miller and Yount, 2002). Structural and stratigraphic relationships of the Bicycle Basin are presented in figures 3 and 4 . The deepest part of the structural basin is north of Bicycle Lake playa at an altitude of about $0 \mathrm{~m}(0 \mathrm{ft})$ NAVD88, or about $701 \mathrm{~m}(2,300 \mathrm{ft})$ below land surface (bls; Densmore and others, 2018). To the north, the basement complex becomes shallower, indicating the existence of one or more east-west trending faults of the Coyote Canyon fault zone. To the south, the basement complex becomes shallower more gradually than to the north.

Tertiary sedimentary deposits include older gravels (Tog) and younger gravels (Tyg; Yount and others, 1994). These deposits are semi-consolidated. Quaternary-Tertiary deposits, divided into Quaternary-Tertiary older alluvium and lacustrine deposits ( $Q T o a$ and $Q T o l$, respectively), overlie the Tertiary sedimentary deposits and underlie Quaternary older and younger deposits. The Quaternary-Tertiary deposits represent a transitional unit from the Pliocene to Pleistocene (Miller and others, 2014); the Quaternary deposits roughly represent the Pleistocene and Holocene (Yount and others, 1994; Schermer and others, 1996). Quaternary-Tertiary older alluvium deposits (QToa) are generally coarse-grained interbedded sands and gravels, whereas Quaternary-Tertiary older lacustrine $(Q T o l)$ are fine-grained sandy clays and silts.
The Quaternary-Tertiary older alluvium (QToa) grades into lakebed clays $(Q T o l)$ north and northwest of Bicycle Lake (dry) playa. Considerable clay, interbedded with sands and gravels, was described for depths of 60-128 m (200-420 ft) bls in geologic logs from wells in this area (14N/3E-23B1-3, $-14 \mathrm{P} 1,-14 \mathrm{P} 2$, and $-23 \mathrm{G} 1)$. These wells are next to and along a dry wash where storm runoff drains to the playa. The presence of extensive clay layers could indicate the wash and lakebed clays most likely were north and northwest of the present playa historically.

Quaternary sedimentary deposits overlie the QuaternaryTertiary deposits and are divided into Quaternary older and younger deposits. The Quaternary younger alluvium ( $Q y a)$ and playa deposits $(Q p)$ (figs. 3, 4), as a group, are composed of unconsolidated sand and gravel with some pedogenic silt and clay. The younger alluvium generally is less than $6 \mathrm{~m}$ (20 ft) thick near the margins of the valley and is generally thicker in the alluvial fans at the foot of Tiefort Mountain, but it could be as thick as $40 \mathrm{~m}(130 \mathrm{ft})$ in the central part of the basin (figs. $4 A, B$ ). The younger alluvium generally lies above the water table; however, it is more permeable than the underlying deposits and, where saturated, is capable of yielding large quantities of water to wells. Quaternary playa deposits (fig. 3; $Q p$ ) underlie the surface of Bicycle Lake (dry) in the southeastern part of the basin. These deposits consist of moderately sorted clay, silt, and fine sand and are as much

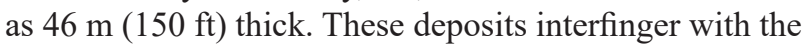
surrounding younger alluvium but generally are above the water table. Because these deposits are fine grained and much less permeable than the younger alluvium, they tend to impede infiltration of surface water, which can pond on the playa after an occasional storm.

\section{Faults}

Several faults have been mapped in the bedrock hills around the Bicycle Basin; the most prominent ones are the Bicycle Lake and Coyote Canyon fault zones (Yount and others, 1994; Schermer and others, 1996; Miller and Yount, 2002; fig. 3). The precise locations of these faults or their splays, where they cross the Bicycle Basin and are buried by sediment, are uncertain. Locations were approximated by projecting mapped faults into the basin and were refined using data collected for this study (including geophysical, InSAR, and gravity surveys and water-level measurements) and inferences from groundwater-flow model calibration. The Bicycle Lake fault zone comprises east-west trending left-lateral faults that cross the southern part of the basin and form the southern boundary of the basin. The Bicycle Lake fault zone uplifts and offsets rocks of the basement complex between Bicycle Basin and Irwin Basin, to the south, and impedes groundwater flow between Bicycle and Irwin Basins. 
A SECTION $A-A^{\prime}$

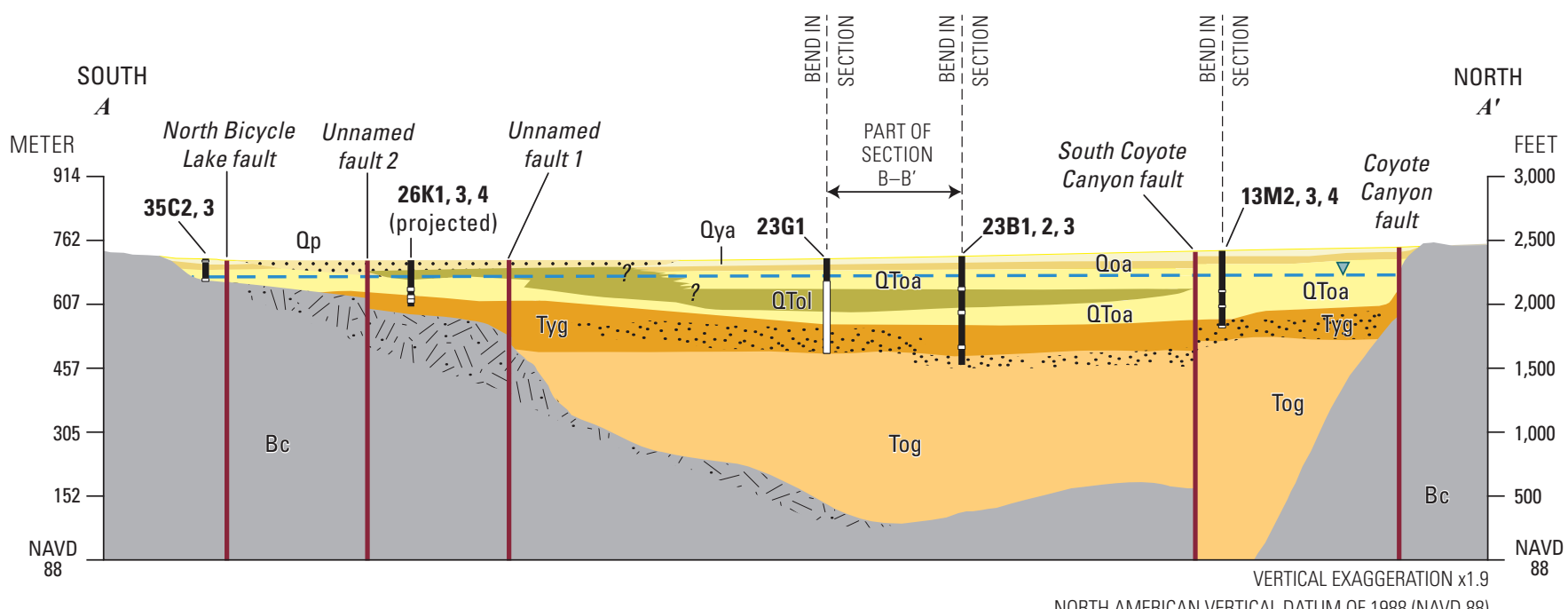
B
SECTION $B-B^{\prime}$

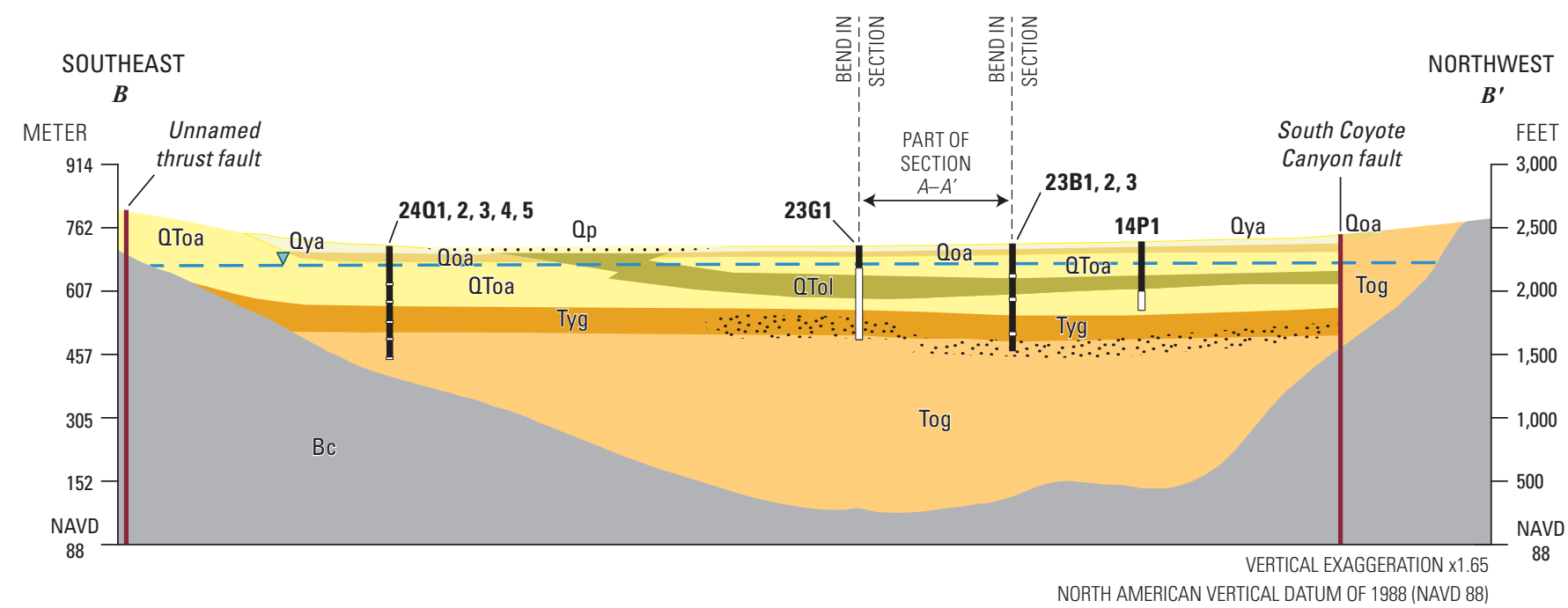

\footnotetext{
Geologic unit/age

$\because$ Playa/Quaternary

Qya Younger alluvium/Quaternary

Qoa Older alluvium/Quaternary

QToa Older alluvium/Quaternary-Tertiary

QTol Older lacustrine/ Quaternary-Tertiary
}

EXPLANATION

Figure 4. Generalized geologic sections across the Bicycle Basin, Fort Irwin National Training Center, California: $A$. A-A', and B. B-B'. (locations of sections are shown on fig. 3). 
One fault splay borders the southern edge of Bicycle Lake (dry) playa and lies just north of wells 14N/3E-35C1-3 (fig. 3). This fault is referred to as the North Bicycle Lake fault by Yount and others (1994). The Coyote Canyon fault zone comprises east-west trending left-lateral faults that cross the northern part of the basin and form the northern boundary of the basin. The Coyote Canyon fault zone appears to uplift Tertiary sedimentary deposits and basement complex on the north end of Bicycle Basin. Either this uplift or the fault zone itself impedes groundwater flow from the Granite Mountains in the north toward Bicycle Basin to the south. On the basis of data collected for this study, an east-west trending fault splay of the Coyote Canyon fault zone was projected into Bicycle Basin, just south of wells 14N/3E-14H1 and 14N/3E-13M1-4, which is referred to as the South Coyote Canyon fault in this report.

Two additional faults were identified in Bicycle Basin on the basis of water-level, geophysical- and gravitysurvey data, and calibration of the groundwater-flow model. Unnamed fault 1 (fig. 3) trends northwest-southeast and is a projected continuation of mapped faults in bedrock areas to the southeast and northwest. Unnamed fault 1 is projected to cross Bicycle Lake playa approximately parallel to the shorter cross runway, which bisects the main runway, and it connects to the southernmost of two northwest-southeast trending parallel faults mapped near the southeastern edge of playa. It is unknown if the northern most of these parallel faults crosses the basin. One multiple-well monitoring site (14N/3E-26K1, 3,4 ) was drilled south of the unnamed fault 1 projection. Water-level altitudes measured in three wells at this site were about 2-6 m (6-20 ft) higher than in wells (14N/3E-24Q5 and $-23 \mathrm{~B} 3$, respectively) north of unnamed fault 1 . Unnamed fault 2 trends west northwest-east southeast and is a projected continuation of mapped faults in Tertiary older alluvium along the west side of the basin.

\section{Land-Surface Deformation Processes}

Land-surface deformation can be found worldwide and is driven by a number of processes, including subsidence due to groundwater withdrawals, as is the case in the San Joaquin Valley of California (for example, Galloway and others, 1999), or land surface uplift, as is the case for aquifer-system recharge (natural or artificial) or clay sediments of a playa swelling after saturation due to inundation. Common forms of land-surface deformation include differential subsidence of compressible deposits resulting from subsurface fluid extraction (for example, water, oil, or natural gas) and rotational stresses caused by and related to movements along faults. A less common form of land-surface deformation involves the formation of giant desiccation cracks (also known as macropolygons) driven by soil desiccation. Surface deformation related to groundwater withdrawal includes land subsidence and localized ground failure due to aquifer-system compaction (for example, Holzer, 2000). Ground failures known as Earth fissures are large tension cracks that form in association with subsidence. Land-surface deformation processes described in this report focus on the mechanics of land subsidence and the development of large surface cracks.

\section{Mechanics of Land Subsidence}

Land subsidence attributed to groundwater pumping is apparent in many aquifer systems that are, at least in part, made up of unconsolidated fine-grained sediments and that have undergone extensive groundwater development (Poland, 1984). The relation between changes in pore-fluid pressure and compression of the aquifer system is based on the principle of effective stress (Terzaghi, 1925):

$$
\sigma_{e}=\sigma_{T}-p
$$

Effective or intergranular stress $\left(\sigma_{e}\right)$ is the difference between total stress or geostatic load $\left(\sigma_{T}\right)$ and the pore-fluid pressure $(p)$. The pore structure of a sedimentary aquifer system is supported by the granular skeleton of the aquifer system and the pore-fluid pressure of the groundwater that fills the intergranular pore space (Meinzer, 1928). If total stress remains constant and groundwater is withdrawn in quantities that result in reduced pore-fluid pressures and water-level declines, the reduction of the pore-fluid pressure increases the intergranular stress, or effective stress, on the aquifer-system skeleton. A change in effective stress deforms the aquifer-system skeleton - that is, an increase in effective stress compresses it, and a decrease in effective stress can cause it to expand. The vertical component of this deformation sometimes results in non-recoverable compaction of the aquifer system and land subsidence. An aquifer-system skeleton that primarily consists of fine-grained sediments, such as silt and clay, is generally much more compressible than one that primarily consists of coarse-grained sediments, such as sand and gravel; however, some of that compression in clay sediments might not be recoverable because of a reorganization of the grain orientations during compaction.

Aquifer-system deformation is small and typically recoverable if the effective stress imposed on the skeleton is less than any previous effective stress (Leake and Prudic, 1991). The greatest historical effective stress imposed on the aquifer system - sometimes the result of the lowest groundwater level - is termed "preconsolidation stress." If the effective stress is greater than the preconsolidation stress, the pore structure of the fine-grained sediments is rearranged; this new configuration results in a reduction of pore volume and, thus, inelastic (largely irreversible) compaction of the aquifer system. Furthermore, the compressibility of the finegrained sediments constituting the aquitards, and any resulting compaction under stresses greater than the preconsolidation stress, is one to two orders of magnitude greater than under stresses less than the preconsolidation stress (Riley, 1998). 
For a developed aquifer-system skeleton that has an appreciable thickness of fine-grained sediments, a large part of the total compaction can be residual compaction (compaction in thick interbedded aquitards and confining units during the typically slow process of fluid-pressure equilibration with the adjacent aquifers; Terzaghi, 1925). Depending on the thickness and the vertical hydraulic diffusivity of a thick interbedded aquitard or confining unit, fluid-pressure equilibration - and thus compaction-lags behind declines in pressure (hydraulic head) in the adjacent aquifers; ultimate compaction could require decades or centuries to approach completion for a given decline in hydraulic head. The time constant, $\tau$, is the time required for about 93 percent of the excess pore pressure to dissipate, and therefore for about 93 percent of the ultimate compaction, following an instantaneous step load, and it is inversely proportional to the vertical hydraulic diffusivity, $K_{v}^{\prime} / S_{s}^{\prime}$, where $K_{v}^{\prime}$ is hydraulic conductivity and $S_{s}^{\prime}$ is specific storage. For a doubly draining aquitard, $\tau$ is also proportional to the square of the half-thickness of the aquitard (Riley, 1969; Riley, 1998):

$$
\tau=S_{s}^{\prime}\left(b^{\prime} / 2\right)^{2} / K_{v}^{\prime}
$$

where

$$
b^{\prime} \quad \text { is the aquitard thickness. }
$$

Ireland and others (1984) estimated that the time constants for aquifer systems at 15 sites in the San Joaquin Valley ranged from 5 to 1,350 years. Numerical modeling based on Terzaghi's theory (1925), which accounts for this time delay, has successfully simulated complex histories of compaction caused by known water-level fluctuations (for example, Helm, 1978; Hanson, 1989; Sneed and Galloway, 2000).

Collectively, these concepts form the aquitard-drainage model (Poland, 1984; Holzer, 1998), which provides the theoretical basis of many successful subsidence studies related to the production of subsurface fluids, including groundwater, oil, and gas. Selected case studies of land subsidence caused by aquifer-system compaction in the United States are presented by Galloway and others (1999). Galloway and Burbey (2011) reviewed measurement, analysis, and modeling of regional land subsidence accompanying groundwater extraction.

\section{Surface Cracking-Earth Fissures, Surface Faults, and Giant Desiccation Cracks of Macropolygon Structure}

Prior research on Earth fissures, surface faults, and giant desiccation cracks indicates several plausible mechanisms for their formation. Common hypotheses include differential compaction of compressible sediments due to subsurface fluid extraction, stresses caused or related to movements along faults (Carpenter, 1993; Burbey, 2010; Holzer, 2010; Galloway and Burbey, 2011), and soil macropolygon formation caused by soil-water desiccation and volumetric contraction of shrink-swell soils (Neal and others, 1968). Given the active and historical groundwater pumping at several wells in Bicycle Basin, along with the observation of macropolygon-patterned ground structure on the southern half of the Bicycle Lake playa, localized differential compaction and soil desiccation are both plausible mechanisms for surface cracking. The implications for mitigation of such ground failures could be quite different because localized differential compaction involves a long-term, deep-subsurface process, whereas soil desiccation can entail a shorter term, near-surface process. Thus, a key objective of this study was to integrate multiple approaches to determine which mechanism is most likely driving the surface cracking at Bicycle Lake playa.

Localized differential compaction, originally proposed by Feth (1951), is described as bending caused by tensile strains generated by locally varying subsidence, and it is often attributed to local variations in aquifer thickness (for example, Holzer, 2010). Jachens and Holzer (1982) corroborated this mechanism for Earth fissure formation on a complex fissure system in alluvium on the east side of the Casa Grande Mountains in south-central Arizona. Their study confirmed that fissures formed where the aquifer system thinned over buried topographic highs of the crystalline bedrock surface. Horizontal strains are at maximum tension above the points of maximum convex-upward curvature on the bedrock surface (Holzer, 2010). Localized differential compaction has been identified in other areas that have arid to semi-arid climates, such as the Las Vegas Valley in Nevada, where Earth fissures are the dominant and most striking type of ground failure associated with groundwater withdrawal (Galloway and others, 1999). Earth fissures, observed in Las Vegas Valley as early as 1925 (Bell and Price, 1991), were not linked directly to subsidence until the late 1950s (Bell, 1981). Most of these Earth fissures are spatially and temporally correlated with groundwater-level declines. These fissures often form preferentially along pre-existing surface features. In the Las Vegas Valley case, these features appear as surface faults in the unconsolidated alluvium (Galloway and others, 1999). The fissures tend to form from the warping of the land surface that results when subsidence is greater on one side of the surface fault than on the other. This differential land subsidence creates tensile stresses that ultimately result in fissuring near zones of maximum warping. Recent work such as Pacheco and others (2006) and Hernandez-Marin and Burbey (2010) provide additional details on these hypotheses for Earth fissure development (fig. 5).

Hypotheses for how macropolygon-patterned ground structures develop as a result of desiccation (fig. 6) date to Neal and others (1968), and more recent work includes Messina and others (2005) and Antrett and others (2012). 
$\boldsymbol{A}$
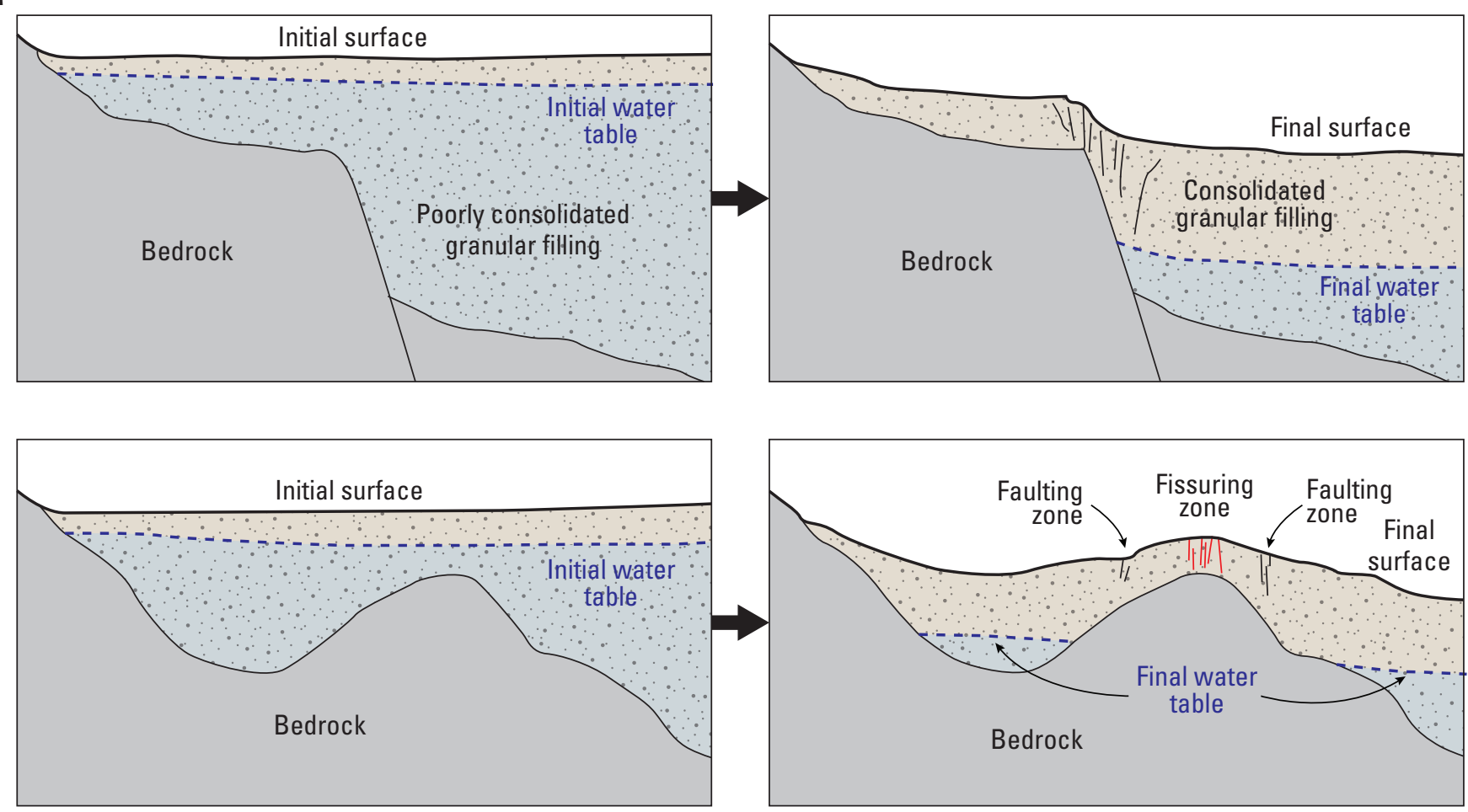

$\boldsymbol{B}$
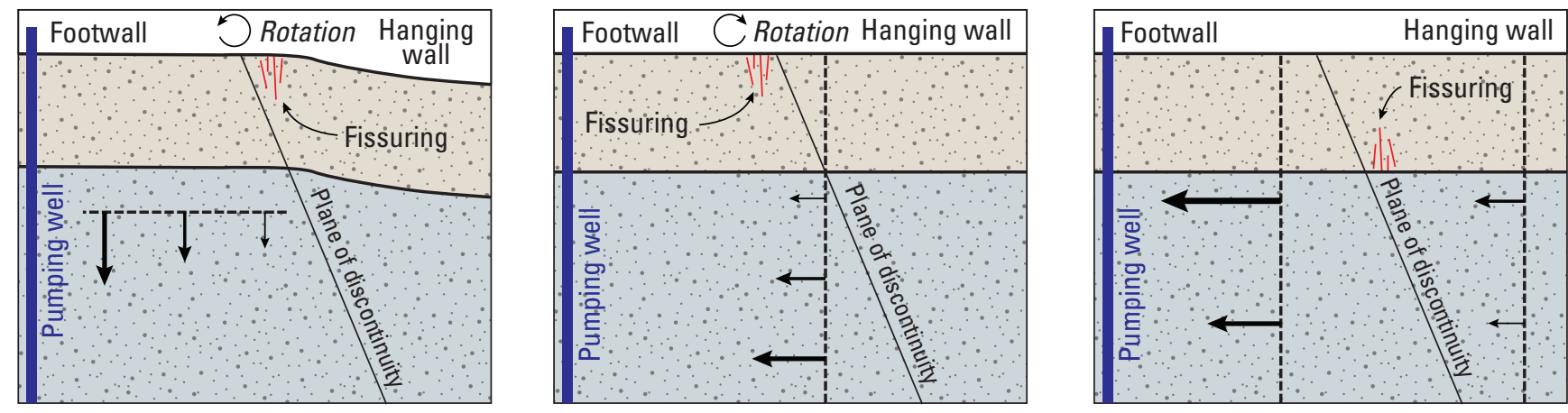

Figure 5. Hypotheses for the formation of Earth fissures and surface faults driven by differential compaction associated with watertable declines from groundwater pumping: $A$, Differential compaction (Pacheco and others, 2006); and $B$, Bending beam, helm, and horizontal discontinuity models (Hernandez-Marin and Burbey, 2010).

Neal and others (1968) studied giant polygonal patterns on 39 playas in Oregon, Nevada, California, Arizona, and New Mexico. They noted that surface cracks were often $5 \mathrm{~m}(16 \mathrm{ft})$ deep and that the coalescing sets of cracks created patterned ground morphology where individual macropolygons attained diameters as large as $300 \mathrm{~m}$ (980 ft). These macropolygons were noted to develop in clay-rich playa settings and were attributed to soil-water desiccation and volumetric change in shrink-swell soils. Because a declining water table leads to decreasing soil moisture in the capillary fringe zone above the water table, volumetric shrinkage results if expansive clay minerals (for example, smectite group) are present in the soil. It is hypothesized that this volumetric contraction in the subsurface ultimately results in soil cracking and the propagation of large cracks up to the surface.

Although brief periods of flooding followed by long periods of evaporation result in wetting and subsequent desiccation of shallow playa soils, the evidence that voids have formed deeper in the soil before a crack is observed at land surface tends to support the hypothesis of volumetric contraction in the subsurface ultimately resulting in soil cracking and propagation of cracks up to the surface. Recent work by El Maarry and others (2012) also supports this hypothesis by indicating that a relatively thick column of desiccating shrink-swell soil is required to build up enough stress to cause the kind of large-scale cracking observed in macropolygon formation at land surface. 
Giant desiccation cracks are commonly distinguished from Earth fissures caused by groundwater pumping by their pattern of complex branching (Harris, 2004; Harris and Allison, 2006). Giant desiccation cracks can be hundreds of meters long and large enough to resemble Earth fissures. Earth fissures generally tend to be longer, straighter, and deeper than giant desiccation cracks. Isolated sink-like depressions at land surface are often the first signs of a giant desiccation crack forming in the subsurface. These sink-like depressions can be partially connected by tunnels at depth, with no crack or depressions apparent at the surface between the features until further subsurface shrinkage and cracking lead to bridging of the features.
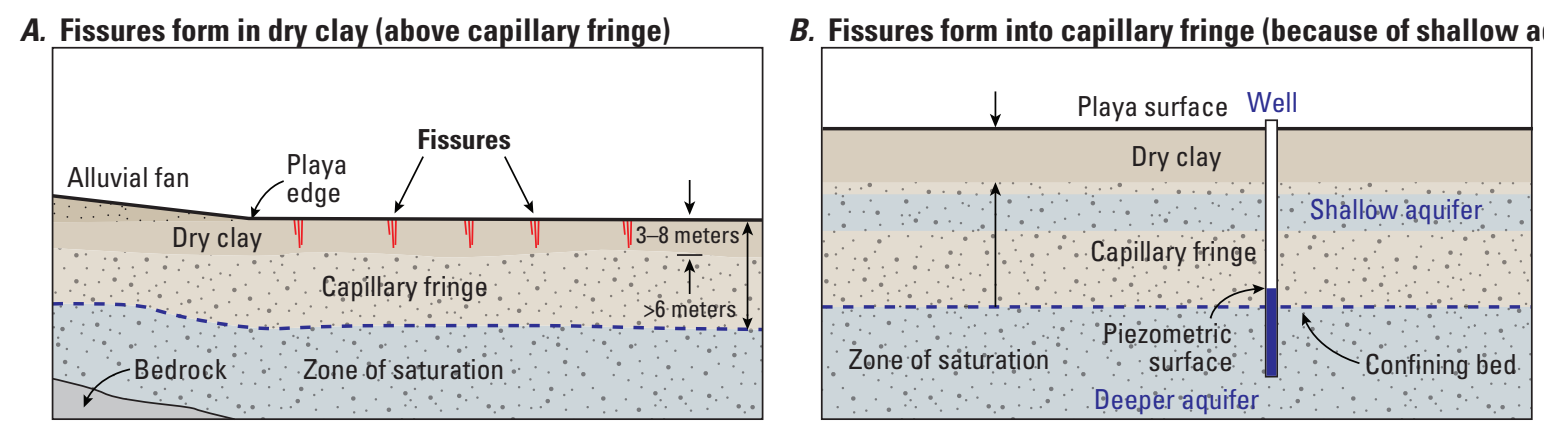

\section{C}

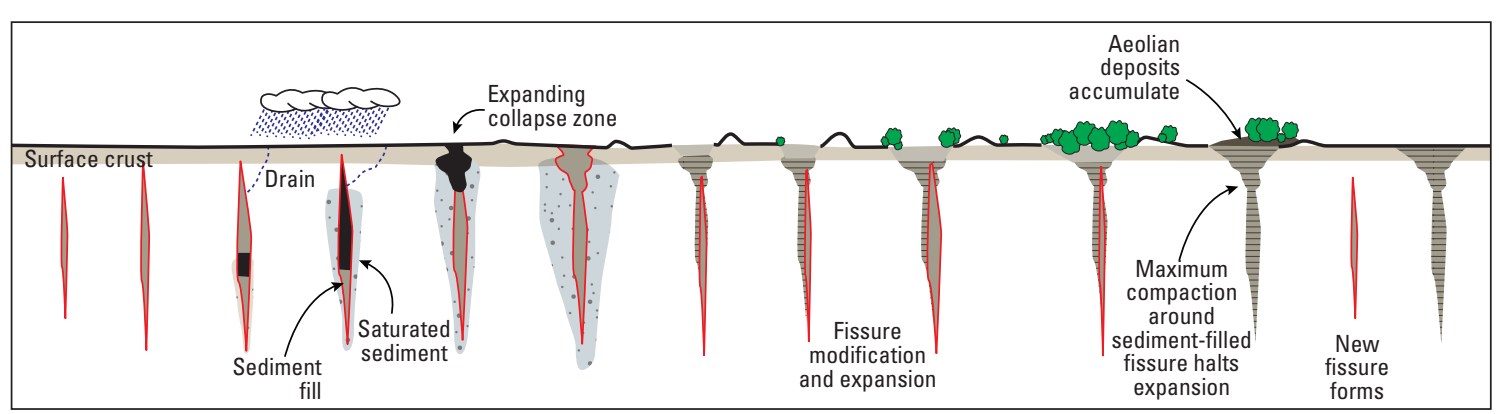

D

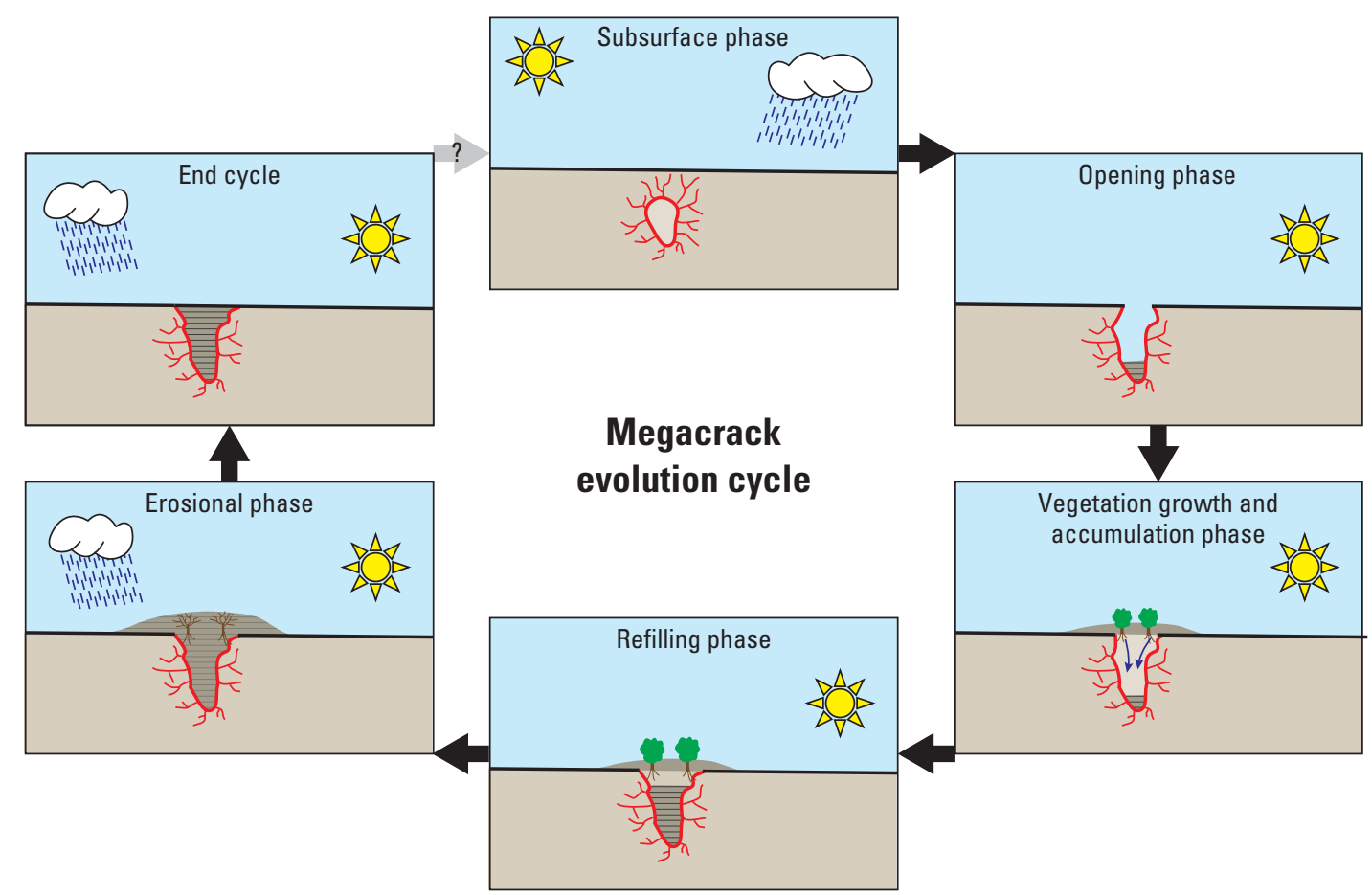

Figure 6. Previous hypotheses for the formation of macropolygon-patterned ground structure found on dry playas throughout the southwest United States: $A$. fissure formation in dry clay (above capillary fringe), from Neal and others (1968); $B$. fissures can form into capillary fringe (because of shallow or perched water table), from Neal and others (1968); C. from Messina and others (2005); and $D$. from Antrett and others (2012). 


\section{Measurements and Methods}

The extent and effects of groundwater declines and landsurface deformation in Bicycle Basin were evaluated using multiple approaches, including (1) measurements of water levels from December 2007 to May 2017, (2) land surveys across the playa, (3) remotely sensed ground displacements detected with InSAR, (4) geophysical surveys of the playa area, and (5) numerical experiments of soil mechanical and cracking processes. These approaches are each described in this section.

\section{Water Levels}

Manual measurements of water levels have been collected sporadically in 35 wells in Bicycle Basin since 1993. Figure 3 shows the location of the monitored wells. Appendix 1 summarizes construction data for these wells. Appendix 2 lists the wells and water-level measurements. Water-level data were also collected in Bicycle Basin every half hour since 2007 using 15 electronic water-level loggers installed in 14 wells and at 1 surface site that provides barometric pressure. All of these data are in the USGS NWIS database and can be accessed at http://waterdata.usgs.gov/ca/ nwis/nwis.

The original water-level loggers contained non-vented, submersible pressure transducers and recorded the absolute pressure in each well open to the atmosphere. The logger at the surface site recorded the barometric pressure. Water levels were calculated for each absolute pressure, corrected for barometric pressure. Corrections to the water-level record include (1) applying offsets for each resetting of the transducer depth as necessary to accommodate declining water levels during the pumping season and (2) instrument-drift corrections using periodic manual water-level measurements for quality control and recalibration of the transducers.

Several gaps exist in the recorded data. Most of these gaps were due to unanticipated increases in pumping rates that resulted in water levels declining below the logger depth. Another gap was caused in one well when the logger suspension line failed. The logger had to be removed and redeployed later. Other minor gaps resulted when measurements were missed either in the well or at the surface site during the time the data were being downloaded. These gaps were generally short, usually no more than a couple hours. More recently, some data gaps have been longer because some logger batteries died between field visits, and funding was not available to replace them. To provide the best coverage in the area of subsidence, some existing loggers were moved from wells outside the area of subsidence to wells inside the area of subsidence. As of 2015, all loggers in Bicycle Basin had been replaced during the previous 2 years.

\section{Land Surveys}

Land surveys were done to monitor vertical and horizontal deformation in subsiding alluvial sediments in
Bicycle Basin. For detailed monitoring, geodetic control was needed across the basin that included a focus on Bicycle Lake (dry) playa. Because no geodetic control existed in Bicycle Basin, a network of monuments was established in 2009 for this study. After establishing the network, land surveys were carried out that included leveling, EDM (electronic distance measurement) surveying, tapex (tape extensometer) measuring, and lidar (light detection and ranging) surveying.

\section{Monument Network Establishment}

A network of monuments was established in Bicycle Basin during January 4-9, 2009. The network consisted of a total of 13 monuments (fig. 7). In an attempt to capture maximum land deformation, the monuments were oriented perpendicular to the 2005-06 crack on the main runway and extended into the area of subsidence identified by InSAR, where a well (BLA4) was drilled. The monuments enable monitoring of multiple displacement components, including vertical and horizontal movement along the transect, to ensure precision of repeat measurements. The goal was to measure sub-millimeter horizontal movement and provide a threedimensional (3-D) representation of any movement across the crack.

Nine monuments were installed along an approximately $4 \mathrm{~km}(2.5 \mathrm{mi})$ transect at a spacing of about $500 \mathrm{~m}(1,500 \mathrm{ft}$; fig. 7). The transect starts at BL1 in volcanic bedrock at the south end of the playa; extends across the playa and crack before bending at BL6 toward the west; and ends at $14 \mathrm{~N} / 3 \mathrm{E}-$ 23B1-3 (BLA4), a multiple-well monitoring site in the area of greatest subsidence (fig. 7). The crack crosses the transect between sites BL4 and BL5. Monuments BL1 and BLA4 are 3 5/8-in.-diameter brass tablets, set flush in drilled holes, and anchored with expanding mortar. Monument BL1 was installed in volcanic bedrock in a presumably stable area near the base of the hills that border the southern end of the playa. The monument at BLA4 was installed in the concrete pad surrounding the vault at multiple-well monitoring site BLA4. Monuments BL2 through BL8 were set in unconsolidated alluvial sediments and are similar to Class B rod marks used by the National Geodetic Survey (Floyd, 1978). These monuments were constructed by first digging a hole about $0.5 \mathrm{~m}(1.5 \mathrm{ft})$ deep and $0.3 \mathrm{~m}(1 \mathrm{ft})$ in diameter. Then, copperclad rods $15-\mathrm{mm}(5 / 8$-in.) in diameter and composed of $1.5-\mathrm{m}$ (4-ft) sections, coupled and then crimped together, were driven

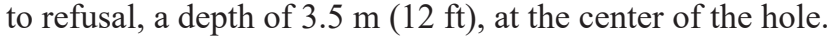
The copper rod was cut off below ground level, and a 92-mm (3 5/8 in.) diameter brass tablet was crimped to the top of the copper rod. The copper rod and brass tablet were enclosed in a 0.15-m (6-in.) diameter, 0.7-m (2-ft) long, polyvinyl chloride (PVC) casing with a lid that closes at land surface to isolate the near-surface part of the rod from thermal expansion and contraction. This PVC casing was anchored in place with about 0.1 cubic meter $\left(\mathrm{m}^{3} ; 3.5\right.$ cubic feet $\left.\left[\mathrm{ft}^{3}\right]\right)$ of concrete. To stabilize the brass tablet for horizontal measurements, the annular space in the conductor casing was filled with sand up to the base of the brass tablet. All brass tablets were labeled with the site designation, and the locations were marked with orange witness posts. 


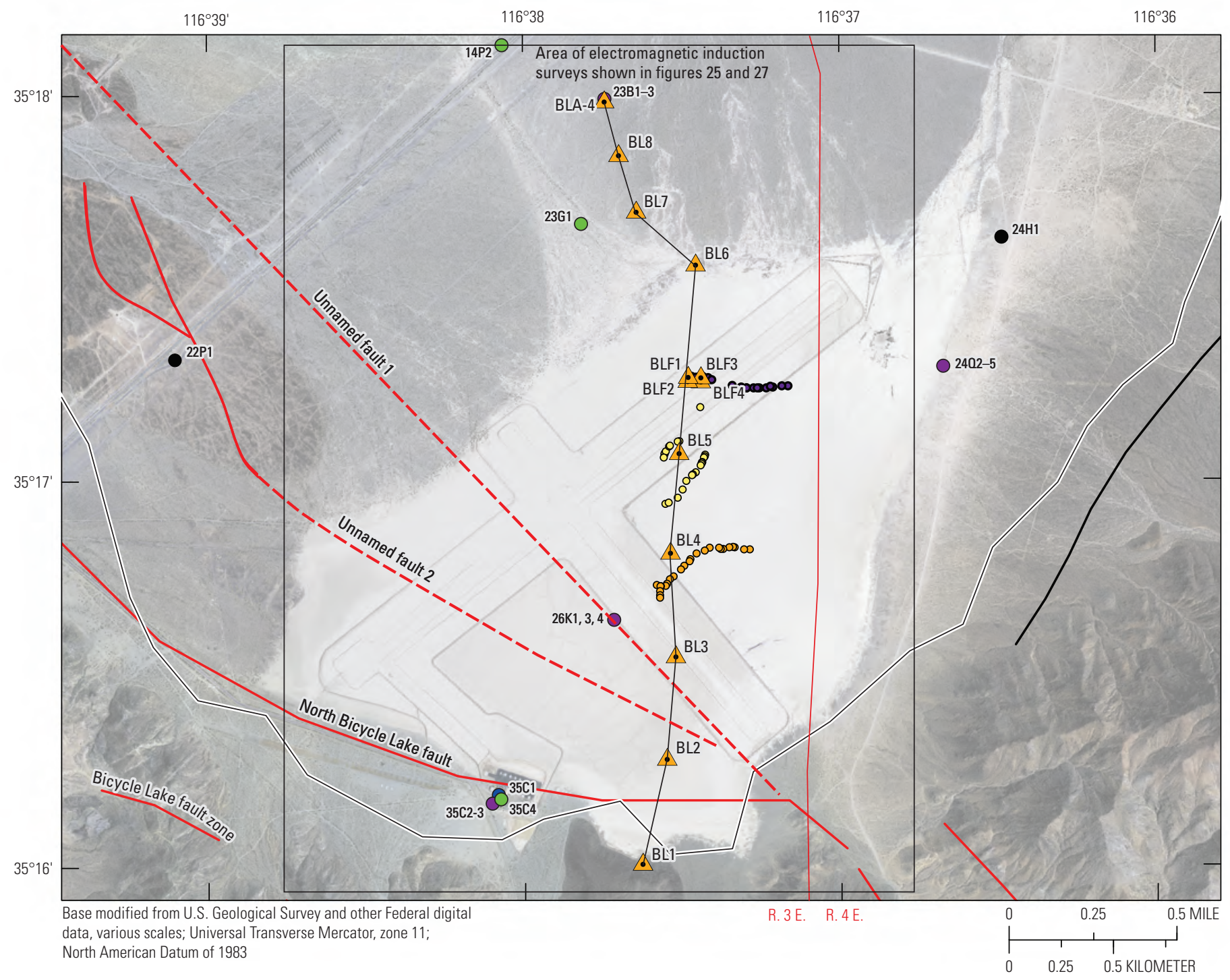

EXPLANATION

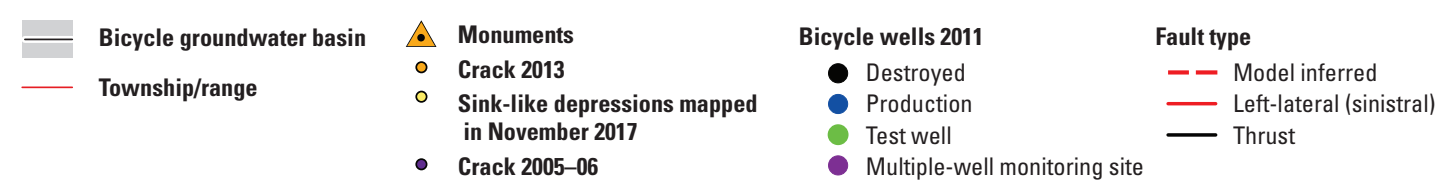

Figure 7. Monument network for geodetic control in Bicycle Basin, Fort Irwin National Training Center, California. 
Four additional monuments were installed at the crack across the main runway and were spaced more closely, at about $18.7 \mathrm{~m}$ (61 ft). Two monuments (BLF2 and BLF4) were placed on the south side of the crack, and two monuments (BLF1 and BLF3) were placed directly across the crack on the north side. Monuments BLF1 through BLF4 are 92-mm (3 5/8 in.) diameter brass tablets installed in the concrete pad of the respective lidar monument. The lidar monuments, BLF1 through BLF4, are vertically oriented PVC casing in a concrete pad above land surface. These monuments have $3.5-\mathrm{m}(12-\mathrm{ft})$ deep anchor rods and incorporate a $0.45 \mathrm{~m}^{3}$ $\left(15 \mathrm{ft}^{3}\right)$ concrete pad from which a $0.15-\mathrm{m}$ (6-in.) diameter

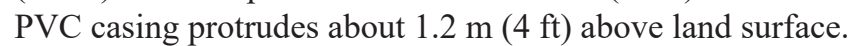
Connected to the anchor rods and passing through the PVC casing is another $15-\mathrm{mm}(5 / 8$-in.) diameter copper-clad rod that extends several centimeters ( $\mathrm{cm}$; more than an inch) above the concrete-filled PVC casing. The exposed copper rod is threaded to allow a $0.45-\mathrm{m}$ (18-in.) diameter sphere to be threaded onto the copper rod on top of the monument for the lidar scans. In addition to the leveling, EDM, and lidar elements of these four monuments, stainless-steel eyebolts were also embedded into the concrete pads as anchors for the tapex, which allows for the comparison of lidar and tapex measurements.

After the network was established, baseline surveys were completed that included second-order, class II geodetic leveling for basin-wide vertical control; EDM measurements for basin-wide horizontal control; and tapex measurements for sub-millimeter horizontal movement across the crack. In addition, terrestrial laser scanning (ground-based, tripodmounted lidar [TLS]) surveys were done to generate a highresolution, 3D image of the crack that allows for 3D modeling of vertical and horizontal components of deformation across the crack.

\section{Procedures}

Leveling surveys for this study provided precise, repeatable, millimeter-scale measurements of vertical changes between the monuments and along the transect. Verticalelevation differences were measured using a Wild NA2 optical level with an optical micrometer plate and matched onepiece 3-m-long Wild GPLE3 stadia rods. The surveys were completed to second-order, class II specifications (National Oceanic Atmospheric Administration, 1984) using methods described in Yamashita and Kaiser (1992). The leveling data were recorded with a handheld computer that does qualityassurance and quality-control processing during the survey using software developed by Yamashita and Yamashita (1992). The surveys were referenced to BL1, which is in an area near the end of the leveling line that lacks notable vertical change. Double-run leveling surveys were done, allowing for a closure error to be calculated for the length of the leveling transect (BL1 to BLA4; fig. 7). The closure error for the baseline (January 2009) survey was less than $0.4 \mathrm{~mm}$ (0.016 in.) for the entire transect length of $3.96 \mathrm{~km}$. Closure errors for subsequent surveys were $1.3 \mathrm{~mm}$ in September 2010, $0.5 \mathrm{~mm}$ in April 2011, $0.1 \mathrm{~mm}$ in October 2014, $0.4 \mathrm{~mm}$ in November 2015, and $0.1 \mathrm{~mm}$ in December 2016. Cumulative errors associated with the rod-scale calibration and the airtemperature-induced refractive indices were assumed to be small for level lines having a stadia distance of less than $5 \mathrm{~km}$ (3.1 mi). Because the transect length was approximately $4 \mathrm{~km}$ long, the cumulative leveling error was comparable to the error of a true first-order class II survey for these distances (Howle and others, 2003).

The EDM surveys provided millimeter-scale measurements of horizontal changes between the monuments along the transect. Slope distances from BL4 to all other monuments were measured using a Hewlett Packard HP3808A EDM, HP retroprisms, and Kern tripods that have centering plumb rods displaying instrument or target height. Setup inaccuracies were estimated to be less than $2 \mathrm{~mm}$ (0.08 in) based on repeated tests. End-point temperature and pressure were recorded at the heights of the instrument and the retroprism using shaded YSI 4600 thermistor thermometers and Wallace and Tiernan aneroid barometers that were calibrated against a Paroscientific 760 pressure standard. Temperature and pressure corrections were applied to the mean value of 10 replications to give a measured slope distance. Using the target height, the instrument height, and the altitudes of the stations, measured slope distances were trigonometrically reduced to horizontal distance.

The tapex measurements provided precise, submillimeterscale measurements of horizontal changes between the monuments across the main-runway crack. The tapex precision is reportedly $0.1 \mathrm{~mm}$ (plus or minus $0.004 \mathrm{in}$.) for spans of $30 \mathrm{~m}(100 \mathrm{ft})$. Slope distances between BLF1 and BLF2 and between BLF3 and BLF4 were periodically measured using a DGSI tapex. The instrument incorporates a built-in digital gage, tensioning collar, and a surveying tape, which has registration pinholes punched at $50.8-\mathrm{mm}$ ( 2 in.) intervals. The tensioning collar properly tensions the tape when the tension control marks are aligned. The digital gage measures the distance from the punched hole in which the registration pin is locked to the reference point in the instrument. The surveying tape was clipped to the eye bolt installed on the vertical face of the concrete pad facing the crack for sites BLF1 and BLF3. The surveying tape was stretched across the crack to the opposing site, in this case BLF2 and BLF4, respectively. 
Ground-based, tripod-mounted lidar (terrestrial laser scanning, or TLS) surveys were used to create high resolution, $3 \mathrm{D}$ laser images of the crack and lidar monuments. TLS is a remote-sensing technology that can collect high-resolution (sub-centimeter), 3-D measurements of the land surface that cannot be achieved by traditional techniques. A laser scanner emits pulses of near-infrared laser light which are timed to measure the distance (range) from the laser scanner to the reflecting surface. Laser ranges are combined with angularorientation data to generate a dense and detailed set of points ( $\mathrm{x}, \mathrm{y}$, and $\mathrm{z}$ locations of individual laser returns) referred to as a point cloud. TLS improves EDM techniques by being able to rapidly generate, transmit, and measure the returning signal in a highly efficient manner, and it is especially useful in low-elevation and low-relief areas because of its ability to detect small changes in land elevation. The sub-centimeter resolution of the point cloud allows for a spatially detailed assessment of topographic change, a quantitative measurement of volumetric changes (erosion or deposition) between datacollection dates, and provides elevation data for modeling of inundation from precipitation runoff. For this study, an Optech 36D laser scanner was mounted on raised and conventional tripods to image a 7,500-square meter $\left(\mathrm{m}^{2} ; 80,700\right.$-square feet, $\mathrm{ft}^{2}$ ) area around the crack. The look-down vantage from the elevated tripod minimized data shadowing in the main-runway crack and small-scale desiccation cracks on the playa as well as shadows cast by the lidar monuments. Multiple scans were collected from different azimuths and look angles to increase the data density to about 10,000 points (lidar returns) per square meter and to cover the entire area of interest. Pointcloud data from the scans were used to produce a composite 3-D image of the study site. This image can be compared to repeat surveys to detect subtle spatial changes in the elevation of the playa surface on either side of the crack and changes in the width, depth, and length of the crack through time. In addition, the spheres of the lidar monuments can be used to assess deformation in the horizontal and vertical planes by mathematically fitting a perfect sphere of a known diameter to the point clouds of the spheres. The fitted spheres also were used to register images. After post-processing, the data points were converted to surface models. The points were then registered using fixed control points in the scan. After processing sequential scanned surfaces of the same area at different times, linear and volumetric changes between the surfaces were measured. Repeat scans were made to see the location of changes and to calculate volumetric and vector changes.

\section{Interferometric Synthetic Aperture Radar}

Use of InSAR is an effective way to measure vertical changes of land surface. InSAR is a satellite-based remotesensing technique that can detect sub-centimeter-level, ground-surface displacements over large areas with a spatial resolution of $90 \mathrm{~m}(270 \mathrm{ft})$ or less. This technique has been used to investigate deformation resulting from earthquakes (Massonnet and others, 1993), volcanoes (Massonnet and others, 1995), and land subsidence (Massonnet and others, 1997; Fielding and others, 1998; Galloway and others, 1998; Amelung and others, 1999, Hoffmann and others, 2001; Galloway and Hoffmann, 2007; Sneed and others, 2013). Synthetic-aperture radar (SAR) imagery is produced by reflecting radar signals off a target area and measuring the twoway travel time back to the satellite. The SAR interferometry technique uses two SAR images of the same area from different times and "interferes" (differences) them, resulting in maps called interferograms that show line-of-sight groundsurface displacement (range change) between the two times. The generation of an interferogram produces two components: amplitude and phase. The amplitude is the measure of the radar signal intensity returned to the satellite and shows buildings, roads, mountains, and other reflective features; the phase is the part of the wavelength reflected back to the satellite and is proportional to the line-of-sight displacement of the ground. If the ground moved away from (subsidence) or toward (uplift) the satellite between the times of the two acquisitions, a slightly different part of the wavelength is reflected back to the satellite, resulting in a measurable phase shift. A map of the phase shifts, or interferogram, can be depicted with a repeating color scale that shows relative range change, where one complete color cycle (fringe) represents one-half the radar wavelength of range change. The indicated range change is between about 83 and 92 percent of truevertical ground motion, depending on the range of typical look angles of the SAR sensor. The direction of changesubsidence or uplift-is indicated by the color progression of the fringe toward the center of a deforming feature. For the interferograms described in the "Results" section, the fringe color progression of blue-green-yellow-orange-red-purple indicates subsidence; the opposite progression indicates uplift.

The InSAR signal quality is partly dependent on satellite position, radar wavelength, atmospheric effects, ground cover, land-use practices, and time span for the interferogram. Strict orbital control is required to precisely control the look angle and position of the satellite. Successful application of the InSAR technique is contingent on looking at the same point on the ground from the same position in space, such that the horizontal distance between each satellite pass, or perpendicular baseline, is minimized. Perpendicular baselines generally greater than about $200 \mathrm{~m}$ (about $660 \mathrm{ft}$ ) produce excessive topographic effects (parallax) that can mask real signal. A digital elevation model (DEM) is used in the interferogram generation process to reduce the topographic effects caused by elevation differences (and also to georeference the image). Phase shifts also can be caused by laterally variable atmospheric conditions, such as clouds or fog, because the non-uniform distribution of water vapor differentially slows the radar signal over an image, which causes a phase shift (Zebker and others, 1997). 
Phase shifts can also be caused by variable atmospheric mass that is associated with different elevations (stratified atmosphere). Atmospheric artifacts can be identified by using several independent interferograms, which are defined as interferograms that do not share a common SAR image. When apparent ground motion is detected only in one interferogram, or a set of interferograms sharing a common SAR image, then the apparent motion likely is due to atmospheric phase delay and can be discounted. The wavelength of the radar affects the signal quality because shorter wavelengths are more sensitive to changes in topography, atmospheric moisture, and deformation. So although the short wavelength requires less deformation to enable measurement using InSAR, it is also more susceptible to errors associated with topographic change and atmospheric moisture.

The type and density of ground cover also can noticeably affect interferogram quality. Densely forested areas are prone to poor signal quality because the radar wavelength cannot effectively penetrate thick vegetation and is either absorbed or reflected back to the satellite from varying depths in the canopy, resulting in incoherent signal (shown as randomized colors on an interferogram). Sparsely vegetated areas and urban centers, however, generally have high signal quality because bare ground, roads, and buildings have high reflectivity and are relatively uniform during at least some range of InSAR timescales. Certain land-use practices, such as farming, also cause incoherent signal return. The tilling and plowing of farm fields causes large and nonuniform ground-surface change that cannot be resolved with InSAR. Signal quality also is adversely affected by longer interferogram time spans, because there is more opportunity for non-uniform change both in urban and in non-urban areas. Many of these error sources were minimized by examining independent interferograms for the target area at Fort Irwin, which is sparsely vegetated and fairly flat. The relatively flat topography of Bicycle Basin enabled the use of C-band interferogram pairs with perpendicular baselines of up to about $580 \mathrm{~m}(1,900 \mathrm{ft})$, and the use of a precise DEM generated from airborne lidar data enabled the use of some X-band interferogram pairs with perpendicular baselines of more than $1,200 \mathrm{~m}(3,900 \mathrm{ft})$ without adversely affecting the ability to interpret the interferograms.

For this study, C-band (56.6 mm, or 2.2 in., wavelength) SAR data from the European Space Agency's European Remote Sensing I and II (ERS-1 and ERS-2), ENVISAT satellite, and Sentinel 1A satellite and X-band (31 mm, or 1.2 in., wavelength) SAR data from the Italian Space Agency's COSMO-SkyMed satellite were used to measure and map range change. The satellites are side-looking, orbit the Earth at an altitude between 620 and $790 \mathrm{~km}$ (380 and $490 \mathrm{mi})$, and have between 12- and 35-day repeat cycles. The angle between vertical and look angle, or the angle of incidence, of the ERS and ENVISAT satellites is about 23 degrees, such that the range change measured by these satellites represents about 92 percent of vertical ground deformation, assuming all the range change resulted from vertical displacement of the land surface. The angle of incidence for the COSMOSkyMed satellite is approximately 34 degrees, such that the measured range change for this satellite is about 83 percent of true-vertical deformation. To enable a direct comparison between the two satellite look angles, range-change values were adjusted to equivalent vertical change by the applicable angle of incidence for each satellite. For this study, 103 interferograms representing periods ranging from 24 days to about 36 months were developed using 18 SAR scenes acquired during 1992-2000 by the ERS-1 and ERS-2 satellites, 54 SAR scenes acquired during $2003-10$ by the ENVISAT satellite, 24 SAR scenes acquired during 2013-14 by the COSMO-SkyMed satellite, and 7 SAR scenes acquired during $2014-15$ by the Sentinel 1A satellite (table 1). A DEM from the USGS National Elevation Dataset was used during processing of C-band SAR data to correct for topographyrelated errors and for georeferencing, and a higher-resolution lidar-generated DEM was used during the processing of the X-band SAR data from 2013 to 2014. The lidar-generated DEM was at a higher resolution to complement the higher sensitivity of the X-band SAR data and also was collected in 2013, which reflected the topography better during the time of the X-band SAR acquisitions.

\section{Surface Geophysical Surveys}

Geophysical surveys are a common approach for imaging subsurface stratigraphy and structure and for characterizing and monitoring geological and hydrological features (Telford and others, 1990). For this study, electrical resistivity tomography (ERT) and electromagnetic induction (EMI) surveys were completed across the Bicycle Lake playa area (fig. $8 \mathrm{~A}$ ) to provide insight on ground failures and to assess the potential utility of these methods as part of a longer term land-surface-deformation monitoring program. Specifically, these two types of surveys were selected for their potential to provide data regarding the depth of the known surface cracks and macropolygon features, as well as to map areas of potential concern for deformation that was otherwise undetectable from ground surveys or remote-sensing imagery.

The use of ERT for imaging subsurface features and structure by measuring the subsurface distribution of electrical resistivity is based on the response of land-surface electrodes to direct electrical current transmitted into the subsurface by a separate set of electrodes. Geophysical imaging by ERT, also termed direct-current resistivity surveying, has been used for many decades in hydrogeological, mining, and geotechnical investigations (Loke, 2004). A variety of electrode-pair configurations can be used for the current transmission and the voltage potential measurement. In modern electrodearray surveys, electrodes are commonly installed at equal spacing in a straight line across the land surface, and a control unit is programmed for automated data collection (fig. $8 B$ ). 
Table 1. Acquisition dates of synthetic aperture radar data, interferogram timelines, and subsidence magnitudes and rates for 103 interferograms analyzed for Bicycle Basin, Fort Irwin National Training Center, California, 1992-2015.

[ERS-1 and ERS-2, European Space Agency's (ESA) European Remote Sensing satellites I and II; ENVISAT, European Space Agency's Environmental Satellite that replaced ERS-1 and ERS-2; in., inch; in. month, inch per month; m, meter; mm, millimeter; mm/dd/yyyy, month/day/year; mm/month, millimeter per month; $x$, period covered;--, not applicable]

\begin{tabular}{|c|c|c|c|c|c|c|c|c|c|c|c|c|}
\hline \multirow{2}{*}{$\begin{array}{l}\text { Interferogram } \\
\text { reference } \\
\text { number }\end{array}$} & \multicolumn{2}{|c|}{ Acquisition dates } & \multirow{2}{*}{$\begin{array}{l}\text { Perpendicular } \\
\text { baseline } \\
(\mathrm{m})\end{array}$} & \multicolumn{2}{|c|}{ Timeline } & \multicolumn{2}{|c|}{ Magnitude } & \multirow{2}{*}{\multicolumn{2}{|c|}{$\frac{\text { Rate }}{\text { (mm/month) (in./month) }}$}} & \multicolumn{3}{|c|}{ Seasonal span of interferogram } \\
\hline & $\begin{array}{c}\text { Start } \\
(\mathrm{mm} / \mathrm{dd} / \mathrm{yyyy})\end{array}$ & $\begin{array}{c}\text { End } \\
(\mathrm{mm} / \mathrm{dd} / \mathrm{yyyy})\end{array}$ & & (days) & (months) & $(\mathrm{mm})$ & (in.) & & & $\begin{array}{c}\text { Annual/ } \\
\text { multi-annual }\end{array}$ & $\begin{array}{c}\text { Winter/spring- } \\
\text { summer/fall }\end{array}$ & $\begin{array}{l}\text { Summer/fall- } \\
\text { winter/spring }\end{array}$ \\
\hline \multicolumn{13}{|c|}{ ERS-1 and ERS-2 } \\
\hline${ }^{1} 1$ & 08/07/1992 & 08/20/1995 & 157 & 1,108 & 36.3 & 26.0 & 1.02 & 0.7 & 0.03 & $\mathrm{x}$ & - & - \\
\hline 12 & $11 / 05 / 1993$ & 09/25/1995 & -172 & 689 & 22.6 & 31.2 & 1.23 & 1.4 & 0.05 & $\mathrm{x}$ & - & - \\
\hline 13 & 01/08/1996 & 07/01/1996 & 99 & 175 & 5.7 & 10.4 & 0.41 & 1.8 & 0.07 & - & $\mathrm{x}$ & - \\
\hline${ }^{14}$ & $10 / 14 / 1996$ & 07/21/1997 & -57 & 280 & 9.2 & 15.6 & 0.61 & 1.7 & 0.07 & $\mathrm{x}$ & - & - \\
\hline 5 & 09/25/1995 & 05/27/1996 & -575 & 245 & 8.0 & 10.4 & 0.41 & 1.3 & 0.05 & - & - & $\mathrm{x}$ \\
\hline${ }^{1} 6$ & 06/16/1997 & 01/12/1998 & -119 & 210 & 6.9 & 10.4 & 0.41 & 1.5 & 0.06 & - & - & $\mathrm{x}$ \\
\hline 7 & 08/05/1996 & 04/07/1997 & 438 & 245 & 8.0 & 10.4 & 0.41 & 1.3 & 0.05 & - & - & $\mathrm{x}$ \\
\hline 8 & 08/05/1996 & 08/10/1998 & 44 & 735 & 24.1 & 31.2 & 1.23 & 1.3 & 0.05 & $\mathrm{x}$ & - & - \\
\hline 9 & 03/03/1997 & 08/25/1997 & 377 & 175 & 5.7 & 15.6 & 0.61 & 2.7 & 0.11 & - & $\mathrm{x}$ & - \\
\hline 10 & 03/03/1997 & 03/23/1998 & 37 & 385 & 12.6 & 26.0 & 1.02 & 2.1 & 0.08 & $\mathrm{x}$ & - & - \\
\hline 11 & 04/07/1997 & 08/25/1997 & -115 & 140 & 4.6 & 10.4 & 0.41 & 2.3 & 0.09 & - & $\mathrm{x}$ & - \\
\hline 12 & 08/25/1997 & 06/01/1998 & -134 & 280 & 9.2 & 10.4 & 0.41 & 1.1 & 0.04 & - & - & $\mathrm{x}$ \\
\hline${ }^{1} 13$ & 03/23/1998 & 08/10/1998 & 61 & 140 & 4.6 & 10.4 & 0.41 & 2.3 & 0.09 & - & $\mathrm{x}$ & - \\
\hline 14 & 03/23/1998 & 06/21/1999 & -5 & 455 & 14.9 & 26.0 & 1.02 & 1.7 & 0.07 & - & $\mathrm{x}$ & - \\
\hline 15 & 07/06/1998 & 03/08/1999 & 87 & 245 & 8.0 & 10.4 & 0.41 & 1.3 & 0.05 & - & - & $\mathrm{x}$ \\
\hline${ }^{1} 16$ & 08/10/1998 & 06/21/1999 & -66 & 315 & 10.3 & 10.4 & 0.41 & 1.0 & 0.04 & $\mathrm{x}$ & - & - \\
\hline${ }^{1} 17$ & 07/26/1999 & $03 / 27 / 2000$ & -144 & 245 & 8.0 & 20.8 & 0.82 & 2.6 & 0.10 & - & - & $\mathrm{x}$ \\
\hline${ }^{1} 18$ & $03 / 27 / 2000$ & 09/18/2000 & 109 & 175 & 5.7 & 15.6 & 0.61 & 2.7 & 0.11 & - & $\mathrm{x}$ & - \\
\hline \multicolumn{13}{|c|}{ ENVISAT } \\
\hline${ }^{1} 19$ & $10 / 26 / 2003$ & $06 / 27 / 2004$ & 74 & 245 & 8.0 & 15.7 & 0.62 & 1.9 & 0.08 & - & - & $\mathrm{x}$ \\
\hline 20 & $10 / 26 / 2003$ & $11 / 14 / 2004$ & 253 & 385 & 12.6 & 31.3 & 1.23 & 2.4 & 0.10 & $\mathrm{x}$ & - & - \\
\hline 21 & $10 / 26 / 2003$ & $12 / 19 / 2004$ & 392 & 420 & 13.8 & 36.5 & 1.44 & 2.5 & 0.10 & $\mathrm{x}$ & - & - \\
\hline 22 & $10 / 26 / 2003$ & $02 / 27 / 2005$ & 181 & 490 & 16.1 & 36.5 & 1.44 & 2.2 & 0.09 & - & - & $\mathrm{x}$ \\
\hline 23 & $10 / 26 / 2003$ & $04 / 03 / 2005$ & -449 & 525 & 17.2 & 52.2 & 2.05 & 2.9 & 0.12 & - & - & $\mathrm{x}$ \\
\hline 24 & $10 / 26 / 2003$ & $06 / 12 / 2005$ & -32 & 595 & 19.5 & 47.0 & 1.85 & 2.3 & 0.09 & - & - & $\mathrm{x}$ \\
\hline 25 & $11 / 30 / 2003$ & $01 / 23 / 2005$ & -109 & 420 & 13.8 & 41.7 & 1.64 & 2.9 & 0.12 & - & - & $\mathrm{x}$ \\
\hline 26 & $11 / 30 / 2003$ & $09 / 25 / 2005$ & -303 & 665 & 21.8 & 62.6 & 2.46 & 2.8 & 0.11 & $\mathrm{x}$ & - & - \\
\hline 27 & $05 / 23 / 2004$ & $01 / 23 / 2005$ & 48 & 245 & 8.0 & 10.4 & 0.41 & 1.2 & 0.05 & $\mathrm{x}$ & - & - \\
\hline
\end{tabular}


Table 1. Acquisition dates of synthetic aperture radar data, interferogram timelines, and subsidence magnitudes and rates for 103 interferograms analyzed for Bicycle Basin, Fort Irwin National Training Center, California, 1992-2015.-Continued

[ERS-1 and ERS-2, European Space Agency's (ESA) European Remote Sensing satellites I and II; ENVISAT, European Space Agency's Environmental Satellite that replaced ERS-1 and ERS-2; in., inch; in./ month, inch per month; m, meter; mm, millimeter; mm/dd/yyyy, month/day/year; mm/month, millimeter per month; x, period covered;-—, not applicable]

\begin{tabular}{|c|c|c|c|c|c|c|c|c|c|c|c|c|}
\hline \multirow{2}{*}{$\begin{array}{l}\text { Interferogram } \\
\text { reference } \\
\text { number }\end{array}$} & \multicolumn{2}{|c|}{ Acquisition dates } & \multirow{2}{*}{$\begin{array}{l}\text { Perpendicular } \\
\text { baseline } \\
\text { (m) }\end{array}$} & \multicolumn{2}{|c|}{ Timeline } & \multicolumn{2}{|c|}{ Magnitude } & \multirow{2}{*}{\multicolumn{2}{|c|}{$\frac{\text { Rate }}{\text { (mm/month) (in./month) }}$}} & \multicolumn{3}{|c|}{ Seasonal span of interferogram } \\
\hline & $\begin{array}{c}\text { Start } \\
\text { (mm/dd/yyyy) }\end{array}$ & $\begin{array}{c}\text { End } \\
\text { (mm/dd/yyyy) }\end{array}$ & & (days) & (months) & $(\mathrm{mm})$ & (in.) & & & $\begin{array}{c}\text { Annual/ } \\
\text { multi-annual }\end{array}$ & $\begin{array}{c}\text { Winter/spring- } \\
\text { summer/fall }\end{array}$ & $\begin{array}{l}\text { Summer/fall- } \\
\text { winter/spring }\end{array}$ \\
\hline \multicolumn{13}{|c|}{ ENVISAT-Continued } \\
\hline 28 & $05 / 23 / 2004$ & $04 / 03 / 2005$ & 454 & 315 & 10.3 & 26.1 & 1.03 & 2.4 & 0.10 & $\mathrm{x}$ & - & - \\
\hline 29 & $05 / 23 / 2004$ & $07 / 17 / 2005$ & 11 & 420 & 13.8 & 31.3 & 1.23 & 2.2 & 0.09 & - & $\mathrm{x}$ & - \\
\hline 30 & $05 / 23 / 2004$ & $09 / 25 / 2005$ & -146 & 490 & 16.1 & 47.0 & 1.85 & 2.8 & 0.12 & - & $\mathrm{x}$ & - \\
\hline 31 & $05 / 23 / 2004$ & $04 / 23 / 2006$ & -99 & 700 & 23.0 & 62.6 & 2.46 & 2.6 & 0.11 & $\mathrm{x}$ & - & - \\
\hline 32 & $06 / 27 / 2004$ & $11 / 14 / 2004$ & 179 & 140 & 4.6 & 10.4 & 0.41 & 2.2 & 0.09 & - & - & $\mathrm{x}$ \\
\hline${ }^{1} 33$ & $06 / 27 / 2004$ & $02 / 27 / 2005$ & 106 & 245 & 8.0 & 26.1 & 1.03 & 3.1 & 0.13 & - & - & $\mathrm{x}$ \\
\hline 34 & $06 / 27 / 2004$ & 03/19/2006 & 311 & 630 & 20.7 & 67.8 & 2.67 & 3.1 & 0.13 & - & - & $\mathrm{x}$ \\
\hline 35 & $11 / 14 / 2004$ & $12 / 19 / 2004$ & 140 & 35 & 1.1 & 0.0 & 0.00 & 0.0 & 0.00 & $\mathrm{x}$ & - & - \\
\hline 36 & $11 / 14 / 2004$ & $02 / 27 / 2005$ & -72 & 105 & 3.4 & 10.4 & 0.41 & 2.9 & 0.12 & - & - & $\mathrm{x}$ \\
\hline 37 & $11 / 14 / 2004$ & $11 / 19 / 2006$ & 9 & 735 & 24.1 & 73.1 & 2.88 & 2.9 & 0.12 & $\mathrm{x}$ & - & - \\
\hline 38 & $01 / 23 / 2005$ & $07 / 17 / 2005$ & -37 & 175 & 5.7 & 15.7 & 0.62 & 2.6 & 0.11 & - & $\mathrm{x}$ & - \\
\hline 39 & $01 / 23 / 2005$ & $09 / 25 / 2005$ & -194 & 245 & 8.0 & 26.1 & 1.03 & 3.1 & 0.13 & - & $\mathrm{x}$ & - \\
\hline${ }^{1} 40$ & $02 / 27 / 2005$ & $06 / 12 / 2005$ & -214 & 105 & 3.4 & 10.4 & 0.41 & 2.9 & 0.12 & - & $\mathrm{x}$ & - \\
\hline 41 & $02 / 27 / 2005$ & 03/19/2006 & 204 & 385 & 12.6 & 47.0 & 1.85 & 3.6 & 0.15 & $\mathrm{x}$ & - & - \\
\hline 42 & $02 / 27 / 2005$ & $11 / 19 / 2006$ & 81 & 630 & 20.7 & 62.6 & 2.46 & 2.9 & 0.12 & - & $\mathrm{x}$ & - \\
\hline 43 & $02 / 27 / 2005$ & $12 / 24 / 2006$ & -180 & 665 & 21.8 & 67.8 & 2.67 & 3.0 & 0.12 & $\mathrm{x}$ & - & - \\
\hline 44 & $04 / 03 / 2005$ & $06 / 12 / 2005$ & 416 & 70 & 2.3 & 5.2 & 0.21 & 2.2 & 0.09 & $\mathrm{x}$ & - & - \\
\hline 45 & $04 / 03 / 2005$ & $10 / 15 / 2006$ & -187 & 560 & 18.4 & 52.2 & 2.05 & 2.7 & 0.11 & - & $\mathrm{x}$ & - \\
\hline 46 & $06 / 12 / 2005$ & $12 / 24 / 2006$ & 33 & 560 & 18.4 & 62.6 & 2.46 & 3.3 & 0.13 & - & - & $\mathrm{x}$ \\
\hline${ }^{1} 47$ & 07/17/2005 & $09 / 25 / 2005$ & -157 & 70 & 2.3 & 10.4 & 0.41 & 4.4 & 0.18 & $\mathrm{x}$ & - & - \\
\hline 48 & $07 / 17 / 2005$ & $04 / 23 / 2006$ & -108 & 280 & 9.2 & 36.5 & 1.44 & 3.8 & 0.16 & - & - & $\mathrm{x}$ \\
\hline${ }^{1} 49$ & $09 / 25 / 2005$ & $04 / 23 / 2006$ & 49 & 210 & 6.9 & 31.3 & 1.23 & 4.4 & 0.18 & - & - & $\mathrm{x}$ \\
\hline${ }^{1} 50$ & 03/19/2006 & $11 / 19 / 2006$ & -123 & 245 & 8.0 & 26.1 & 1.03 & 3.1 & 0.13 & - & $\mathrm{x}$ & - \\
\hline 51 & $10 / 15 / 2006$ & $01 / 28 / 2007$ & -41 & 105 & 3.4 & 15.7 & 0.62 & 4.4 & 0.18 & - & - & $\mathrm{x}$ \\
\hline 52 & $10 / 15 / 2006$ & 03/04/2007 & 199 & 140 & 4.6 & 15.7 & 0.62 & 3.3 & 0.13 & - & - & $\mathrm{x}$ \\
\hline${ }^{1} 53$ & $10 / 15 / 2006$ & $02 / 17 / 2008$ & 33 & 490 & 16.1 & 36.5 & 1.44 & 2.2 & 0.09 & - & - & $\mathrm{x}$ \\
\hline 54 & $12 / 24 / 2006$ & $01 / 13 / 2008$ & -238 & 385 & 12.6 & 31.3 & 1.23 & 2.4 & 0.10 & $\mathrm{x}$ & - & - \\
\hline 55 & $12 / 24 / 2006$ & 08/30/2009 & -218 & 980 & 32.1 & 78.3 & 3.08 & 2.3 & 0.10 & - & $\mathrm{x}$ & - \\
\hline
\end{tabular}


Table 1. Acquisition dates of synthetic aperture radar data, interferogram timelines, and subsidence magnitudes and rates for 103 interferograms analyzed for Bicycle Basin, Fort Irwin National Training Center, California, 1992-2015.-Continued

[ERS-1 and ERS-2, European Space Agency's (ESA) European Remote Sensing satellites I and II; ENVISAT, European Space Agency's Environmental Satellite that replaced ERS-1 and ERS-2; in., inch; in. month, inch per month; m, meter; mm, millimeter; mm/dd/yyyy, month/day/year; mm/month, millimeter per month; $\mathrm{x}$, period covered;-, not applicable]

\begin{tabular}{|c|c|c|c|c|c|c|c|c|c|c|c|c|}
\hline \multirow{2}{*}{$\begin{array}{l}\text { Interferogram } \\
\text { reference } \\
\text { number }\end{array}$} & \multicolumn{2}{|c|}{ Acquisition dates } & \multirow{2}{*}{$\begin{array}{l}\text { Perpendicular } \\
\text { baseline } \\
\text { (m) }\end{array}$} & \multicolumn{2}{|c|}{ Timeline } & \multicolumn{2}{|c|}{ Magnitude } & \multirow{2}{*}{\multicolumn{2}{|c|}{$\frac{\text { Rate }}{\text { (mm/month) (in./month) }}$}} & \multicolumn{3}{|c|}{ Seasonal span of interferogram } \\
\hline & $\begin{array}{c}\text { Start } \\
\text { (mm/dd/yyyy) }\end{array}$ & $\begin{array}{c}\text { End } \\
\text { (mm/dd/yyyy) }\end{array}$ & & (days) & (months) & $(\mathrm{mm})$ & (in.) & & & $\begin{array}{c}\text { Annual/ } \\
\text { multi-annual }\end{array}$ & $\begin{array}{l}\text { Winter/spring- } \\
\text { summer/fall }\end{array}$ & $\begin{array}{l}\text { Summer/fall- } \\
\text { winter/spring }\end{array}$ \\
\hline \multicolumn{13}{|c|}{ ENVISAT_Continued } \\
\hline 56 & $01 / 28 / 2007$ & $02 / 17 / 2008$ & 74 & 385 & 12.6 & 26.1 & 1.03 & 2.0 & 0.08 & $\mathrm{x}$ & - & - \\
\hline 57 & 03/04/2007 & $02 / 17 / 2008$ & -172 & 350 & 11.5 & 26.1 & 1.03 & 2.2 & 0.09 & $\mathrm{x}$ & - & - \\
\hline 58 & $08 / 26 / 2007$ & 07/26/2009 & -78 & 700 & 23.0 & 52.2 & 2.05 & 2.2 & 0.09 & $\mathrm{x}$ & - & - \\
\hline 59 & 01/13/2008 & 08/10/2008 & 15 & 210 & 6.9 & 15.7 & 0.62 & 2.2 & 0.09 & - & $\mathrm{x}$ & - \\
\hline 60 & $01 / 13 / 2008$ & 05/17/2009 & -173 & 490 & 16.1 & 26.1 & 1.03 & 1.6 & 0.06 & $\mathrm{x}$ & - & - \\
\hline 61 & $02 / 17 / 2008$ & 09/14/2008 & -43 & 210 & 6.9 & 20.9 & 0.82 & 2.9 & 0.12 & - & $\mathrm{x}$ & - \\
\hline${ }^{1} 62$ & $02 / 17 / 2008$ & 03/08/2009 & 242 & 385 & 12.6 & 26.1 & 1.03 & 2.0 & 0.08 & $\mathrm{x}$ & - & - \\
\hline 63 & $02 / 17 / 2008$ & 04/12/2009 & -6 & 420 & 13.8 & 31.3 & 1.23 & 2.2 & 0.09 & $\mathrm{x}$ & - & - \\
\hline 64 & 08/10/2008 & 05/17/2009 & -188 & 280 & 9.2 & 15.7 & 0.62 & 1.6 & 0.07 & - & - & $\mathrm{x}$ \\
\hline 65 & 09/14/2008 & 05/17/2009 & 224 & 245 & 8.0 & 10.4 & 0.41 & 1.2 & 0.05 & - & - & $\mathrm{x}$ \\
\hline 66 & 03/08/2009 & 05/02/2010 & -154 & 420 & 13.8 & 15.7 & 0.62 & 1.1 & 0.04 & $\mathrm{x}$ & - & - \\
\hline${ }^{1} 67$ & 03/08/2009 & 08/15/2010 & 94 & 525 & 17.2 & 20.9 & 0.82 & 1.2 & 0.05 & - & $\mathrm{x}$ & - \\
\hline 68 & 04/12/2009 & 05/02/2010 & 94 & 385 & 12.6 & 15.7 & 0.62 & 1.2 & 0.05 & $\mathrm{x}$ & - & - \\
\hline 69 & $05 / 17 / 2009$ & 05/02/2010 & -93 & 350 & 11.5 & 15.7 & 0.62 & 1.3 & 0.05 & $\mathrm{x}$ & - & - \\
\hline 70 & 05/17/2009 & 08/15/2010 & 155 & 455 & 14.9 & 26.1 & 1.03 & 1.7 & 0.07 & - & $\mathrm{x}$ & - \\
\hline 71 & 08/30/2009 & 08/15/2010 & -50 & 350 & 11.5 & 15.7 & 0.62 & 1.3 & 0.05 & $\mathrm{x}$ & - & - \\
\hline 72 & 05/02/2010 & 08/15/2010 & 248 & 105 & 3.4 & 10.4 & 0.41 & 2.9 & 0.12 & - & $\mathrm{x}$ & - \\
\hline \multicolumn{13}{|c|}{ COSMO-SkyMed } \\
\hline 73 & $06 / 20 / 2013$ & 08/07/2013 & -347 & 48 & 1.6 & 0.0 & 0.00 & 0.0 & 0.00 & - & $\mathrm{x}$ & - \\
\hline 74 & $06 / 20 / 2013$ & $08 / 23 / 2013$ & -392 & 64 & 2.1 & 0.0 & 0.00 & 0.0 & 0.00 & - & $\mathrm{x}$ & - \\
\hline 75 & $06 / 20 / 2013$ & $12 / 13 / 2013$ & 596 & 176 & 5.8 & 0.0 & 0.00 & 0.0 & 0.00 & - & - & $\mathrm{x}$ \\
\hline 76 & $06 / 20 / 2013$ & $04 / 20 / 2014$ & $-1,132$ & 304 & 10.0 & 7.7 & 0.30 & 0.8 & 0.03 & - & - & $\mathrm{x}$ \\
\hline 177 & $06 / 20 / 2013$ & $06 / 23 / 2014$ & -149 & 368 & 12.1 & 10.3 & 0.39 & 0.8 & 0.03 & $\mathrm{x}$ & - & - \\
\hline 78 & 08/07/2013 & 09/24/2013 & -271 & 48 & 1.6 & 0.0 & 0.00 & 0.0 & 0.00 & - & $\mathrm{x}$ & - \\
\hline 79 & 08/07/2013 & $10 / 10 / 2013$ & -280 & 64 & 2.1 & 0.0 & 0.00 & 0.0 & 0.00 & - & - & $\mathrm{x}$ \\
\hline 80 & 08/07/2013 & $12 / 13 / 2013$ & 947 & 128 & 4.2 & 0.0 & 0.00 & 0.0 & 0.00 & - & - & $\mathrm{x}$ \\
\hline 81 & 08/07/2013 & $01 / 14 / 2014$ & 298 & 160 & 5.2 & 0.0 & 0.00 & 0.0 & 0.00 & - & - & $\mathrm{x}$ \\
\hline 82 & 08/07/2013 & $06 / 23 / 2014$ & 197 & 320 & 10.5 & 10.3 & 0.39 & 1.0 & 0.04 & $\mathrm{x}$ & - & - \\
\hline
\end{tabular}


Table 1. Acquisition dates of synthetic aperture radar data, interferogram timelines, and subsidence magnitudes and rates for 103 interferograms analyzed for Bicycle Basin, Fort Irwin National Training Center, California, 1992-2015.-Continued

[ERS-1 and ERS-2, European Space Agency's (ESA) European Remote Sensing satellites I and II; ENVISAT, European Space Agency's Environmental Satellite that replaced ERS-1 and ERS-2; in., inch; in./ month, inch per month; m, meter; mm, millimeter; mm/dd/yyyy, month/day/year; mm/month, millimeter per month; x, period covered;-—, not applicable]

\begin{tabular}{|c|c|c|c|c|c|c|c|c|c|c|c|c|}
\hline \multirow{2}{*}{$\begin{array}{l}\text { Interferogram } \\
\text { reference } \\
\text { number }\end{array}$} & \multicolumn{2}{|c|}{ Acquisition dates } & \multirow{2}{*}{$\begin{array}{l}\text { Perpendicular } \\
\text { baseline } \\
\text { (m) }\end{array}$} & \multicolumn{2}{|c|}{ Timeline } & \multicolumn{2}{|c|}{ Magnitude } & \multicolumn{2}{|c|}{ Rate } & \multicolumn{3}{|c|}{ Seasonal span of interferogram } \\
\hline & $\begin{array}{c}\text { Start } \\
\text { (mm/dd/yyyy) }\end{array}$ & $\begin{array}{c}\text { End } \\
\text { (mm/dd/yyyy) }\end{array}$ & & (days) & (months) & (mm) & (in.) & (mm/month & n./month) & $\begin{array}{c}\text { Annual/ } \\
\text { multi-annual }\end{array}$ & $\begin{array}{c}\text { Winter/spring- } \\
\text { summer/fall }\end{array}$ & $\begin{array}{l}\text { Summer/fall- } \\
\text { winter/spring }\end{array}$ \\
\hline \multicolumn{13}{|c|}{ COSMO-SkyMed-Continued } \\
\hline 83 & $08 / 23 / 2013$ & $12 / 13 / 2013$ & 987 & 112 & 3.7 & 0.0 & 0.00 & 0.0 & 0.00 & - & - & $\mathrm{x}$ \\
\hline 84 & $08 / 23 / 2013$ & $04 / 20 / 2014$ & -741 & 240 & 7.9 & 7.7 & 0.30 & 1.0 & 0.04 & - & - & $\mathrm{x}$ \\
\hline 85 & $08 / 23 / 2013$ & $06 / 23 / 2014$ & 242 & 304 & 10.0 & 10.3 & 0.39 & 1.0 & 0.04 & $\mathrm{x}$ & - & - \\
\hline 86 & $09 / 24 / 2013$ & $04 / 20 / 2014$ & -514 & 208 & 6.8 & 2.6 & 0.10 & 0.4 & 0.01 & - & - & $\mathrm{x}$ \\
\hline 87 & $09 / 24 / 2013$ & $06 / 23 / 2014$ & 472 & 272 & 8.9 & 7.7 & 0.30 & 0.8 & 0.03 & $\mathrm{x}$ & - & - \\
\hline 88 & $10 / 10 / 2013$ & $12 / 13 / 2013$ & 1,223 & 64 & 2.1 & 0.0 & 0.00 & 0.0 & 0.00 & - & - & $\mathrm{x}$ \\
\hline 89 & $10 / 10 / 2013$ & 03/19/2014 & 831 & 160 & 5.2 & 0.0 & 0.00 & 0.0 & 0.00 & - & - & $\mathrm{x}$ \\
\hline 90 & $10 / 10 / 2013$ & $04 / 20 / 2014$ & -505 & 192 & 6.3 & 5.1 & 0.20 & 0.8 & 0.03 & - & - & $\mathrm{x}$ \\
\hline 91 & $10 / 10 / 2013$ & $06 / 23 / 2014$ & 478 & 256 & 8.4 & 10.3 & 0.39 & 1.2 & 0.05 & $\mathrm{x}$ & - & - \\
\hline 92 & $12 / 13 / 2013$ & $06 / 23 / 2014$ & -746 & 192 & 6.3 & 7.7 & 0.30 & 1.2 & 0.05 & - & $\mathrm{x}$ & - \\
\hline 93 & $01 / 14 / 2014$ & 03/19/2014 & 253 & 64 & 2.1 & 0.0 & 0.00 & 0.0 & 0.00 & $\mathrm{x}$ & - & - \\
\hline 94 & $01 / 14 / 2014$ & $06 / 23 / 2014$ & -100 & 160 & 5.2 & 10.3 & 0.39 & 1.9 & 0.08 & - & $\mathrm{x}$ & - \\
\hline 95 & 03/19/2014 & $06 / 23 / 2014$ & -354 & 96 & 3.1 & 5.1 & 0.20 & 1.6 & 0.06 & - & $\mathrm{x}$ & - \\
\hline 96 & $04 / 20 / 2014$ & $06 / 23 / 2014$ & 982 & 64 & 2.1 & 5.1 & 0.20 & 2.4 & 0.09 & - & $\mathrm{x}$ & - \\
\hline \multicolumn{13}{|c|}{ Sentinel $1 \mathrm{~A}$} \\
\hline 97 & $10 / 29 / 2014$ & $11 / 22 / 2014$ & 26 & 24 & 0.8 & 0.0 & 0.00 & 0.0 & 0.00 & - & - & $\mathrm{x}$ \\
\hline 98 & $10 / 29 / 2014$ & $12 / 16 / 2014$ & -95 & 48 & 1.6 & 4.7 & 0.19 & 3.0 & 0.12 & - & - & $\mathrm{x}$ \\
\hline 99 & $10 / 29 / 2014$ & $05 / 09 / 2015$ & -8 & 192 & 6.3 & 9.4 & 0.37 & 1.5 & 0.06 & - & - & $\mathrm{x}$ \\
\hline 100 & $10 / 29 / 2014$ & 09/06/2015 & -65 & 312 & 10.2 & 9.4 & 0.37 & 0.9 & 0.04 & $\mathrm{x}$ & - & - \\
\hline${ }^{1} 101$ & $10 / 29 / 2014$ & $10 / 24 / 2015$ & 69 & 360 & 11.8 & 14.1 & 0.56 & 1.2 & 0.05 & $\mathrm{x}$ & - & - \\
\hline 102 & $01 / 09 / 2015$ & $10 / 24 / 2015$ & 85 & 288 & 9.4 & 4.7 & 0.19 & 0.5 & 0.02 & - & $\mathrm{x}$ & - \\
\hline 103 & $03 / 22 / 2015$ & $11 / 17 / 2015$ & -58 & 240 & 7.9 & 14.1 & 0.56 & 1.8 & 0.07 & $\mathrm{x}$ & - & - \\
\hline
\end{tabular}

${ }^{1}$ Used for the time series generation. 

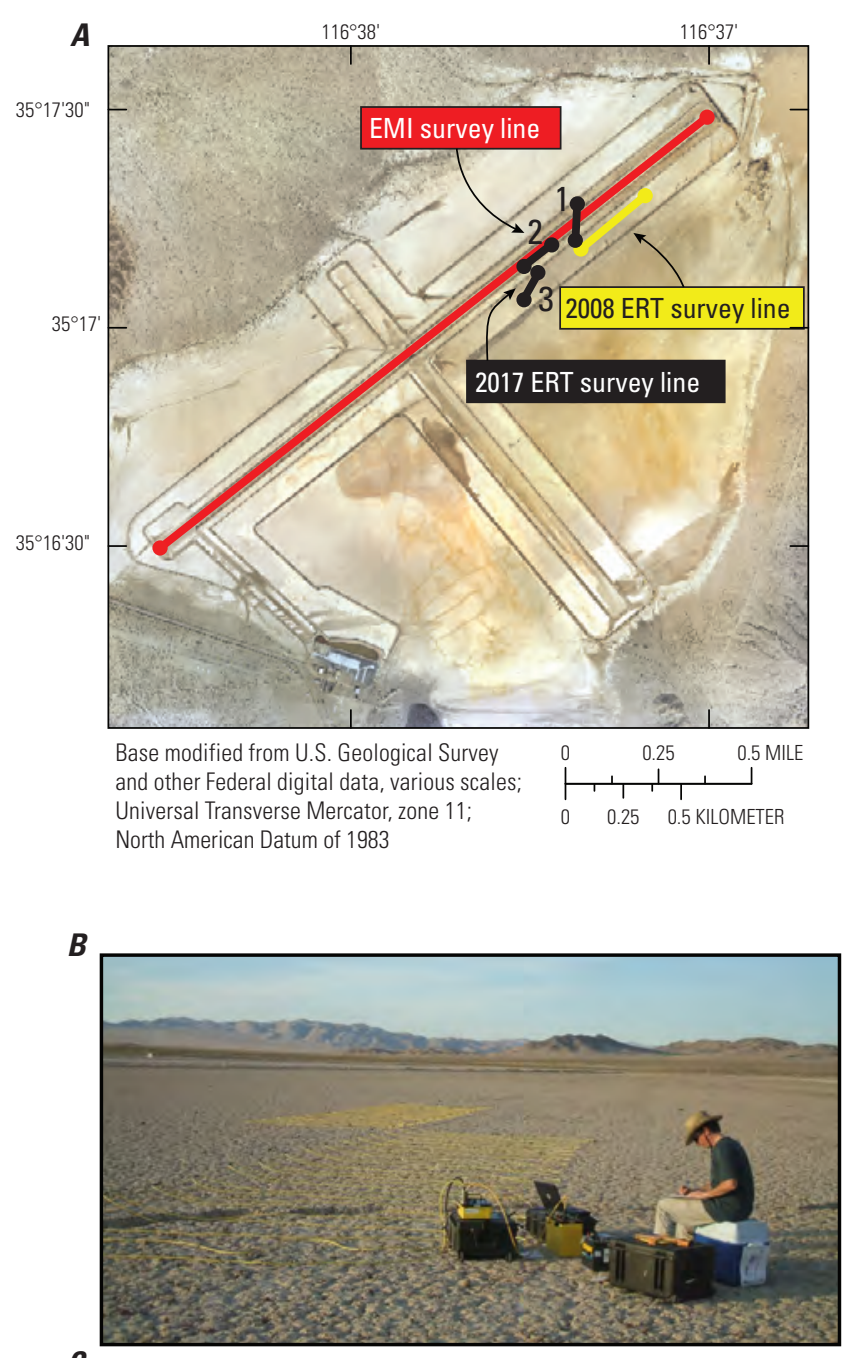

C

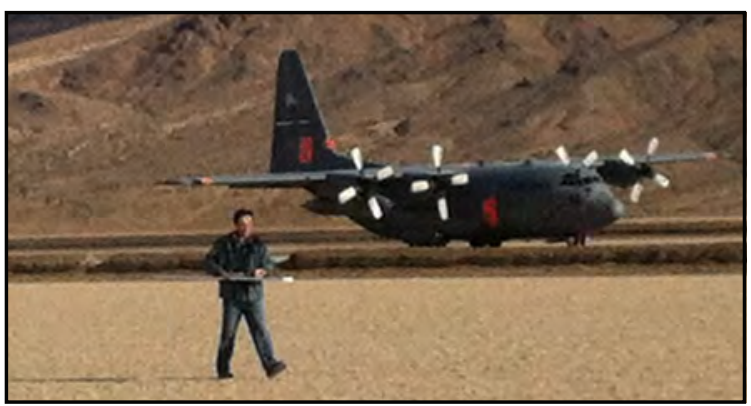

Figure 8. Geophysical survey data-collection surveys: $A$, the location of geophysical survey lines along the main runway and the 2005-06 crack area; $B$, electrical resistivity tomography (ERT) data collection and electrode array for the shallow survey (1-meter electrode spacing) across the crack in 2007; and $C$, electromagnetic induction (EMI) data collection from the survey in December 2014.
The current and resulting voltage potential are measured for numerous electrode-pair spacings, and the subsurface distribution of apparent electrical resistivity is calculated. From the current $(I)$ and voltage $(V)$ values, apparent resistivity $\left(p_{a}\right)$ is calculated using Ohm's Law:

$$
p_{a}=k V / I
$$

where

$$
\begin{aligned}
& k \quad \text { is the geometric factor, which depends } \\
& \text { on the arrangement of the electrodes } \\
& \text { (Loke, 2004). }
\end{aligned}
$$

Resistivity meters normally give a resistance value, $R=V / I$, so in practice, the apparent resistivity value is calculated as follows:

$$
p_{a}=k R
$$

The apparent resistivity value is not the true resistivity of the subsurface, but rather a value that assumes homogeneity of the subsurface. Inverse-modeling techniques transform the measured apparent resistivity to an optimal estimate of the actual subsurface resistivity distribution. The technique of ERT uses this process to provide discrete two-dimensional (2-D) slice profiles of the subsurface underlying the electrode survey line, or additional surveys can be combined for 3-D imaging. The variability of resistivity for different soils and rock types makes this method highly effective for subsurface imaging (Daniels and Alberty, 1966; Keller and Frischknecht, 1966; Loke, 2004). Resistivities of alluvium generally range from 10 to 800 ohm-m $(\Omega-\mathrm{m})$, whereas clay-rich sediments often range from 1 to $100 \Omega-\mathrm{m}$. Resistivity of porous media such as soils and rocks also varies depending on the porosity, the degree of water saturation, and the concentration of solutes in the pore water. Such variations in resistivity provide the basis for imaging the depth of the crack across the main runway.

To image crack depth, the survey design was to install the electrode array centered on the crack in the main-runway area and then to increase the electrode spacing in subsequent surveys to resolve the depth of the crack (fig. $8 A$ ). Depth of investigation is primarily determined by the spacing of individual electrode pairs in the array. By using a dipoledipole array geometry (Telford and others, 1990) and varying the electrode spacing from 1 to $4 \mathrm{~m}(3.3$ to $13.1 \mathrm{ft})$ in sequential surveys, the depth of investigation for this study varied from about 11 to $46 \mathrm{~m}$ (36 to $150 \mathrm{ft}$ ) at the mainrunway crack area for surveys completed during 2008. Three additional ERT surveys were carried out in 2017 (fig. 8A): 
(1) one at the main-runway crack, but oriented more northsouth than the 2008 surveys; (2) one in an area of potential concern for deformation (as indicated by the EMI surveys) southwest of the runway crack; and (3) one across a healed giant desiccation crack associated with a macropolygon feature, based on assessment using aerial imagery. In all the ERT surveys, data sampling and collection were controlled by using an AGI SuperSting R8 system. The field data were subsequently inverse modeled, using the RES2D software program (Loke, 2004) for surveys from 2008 and the AGI EarthImager software for surveys from 2017, and the results were presented as a series of 2-D slice profiles of subsurfaceresistivity distribution (Thayer and others, 2018, Dintaman and others, 2019).

As with ERT, the EMI method of ground conductivity mapping has been used for decades in environmental and engineering applications (Reynolds, 2011). The EMI instruments comprise two or more sets of coils that have various spacing between the transmitter and receiver coils. An electrical current through the transmitter coil is used to generate an electromagnetic field, known as the primary field, at specific operating frequencies. Eddy currents generated in the ground below the transmitted field cause secondary electrical currents to be generated in the conductive materials within its influence. The induced secondary field is measured by the receiver coil, and the magnitude of this field is separated into two orthogonal components (in-phase and quadrature) to provide data on both the apparent electrical conductivity and the magnetic susceptibility of the subsurface material. Analysis of in-phase and quadrature components helps determine if changes in the observed secondary field are the result of variability in apparent electrical conductivity driven by soil properties, rather than a response to buried metallic objects.

For this study, EMI was used to detect areas of potential concern for ground failures. Survey lines were run repeatedly along the entire length of the main runway (fig. $8 \mathrm{~A}$ ) because of concern for aircraft operations. Numerous additional survey lines were run across the broader Bicycle Lake playa area (not shown in fig. 8) to map anomalies related to differences in soil type and variability in soil-moisture content that could be indicative of subsurface cracking for which there was no apparent expression at land surface. The EMI surveys were done in 2008, 2014, and 2015 using a Geophex GEM-2 sensor, which is a shoulder-mounted, multi-frequency broadband electromagnetic sensor (fig. 8C). The depth of investigation for EMI instruments with fixed coil spacing is largely a function of the operating frequency, such that lower frequency transmissions provide greater penetration and thus deeper subsurface imaging compared to higher frequencies (Won and others, 1996; Huang, 2005). The frequencies used in this study ranged from about 3 to 47 kilohertz $(\mathrm{kHz})$. All results presented are from the $18 \mathrm{kHz}$ frequency response, however, because all measured frequencies gave very similar results in this study as a result of the relative similarity of the shallow soil type in the study area. The depth of investigation for the EMI surveys was estimated to be no more than a few meters, given the operating frequencies and the relatively high electrical conductivity of the playa soils (Won and others, 1996; Huang, 2005). A GPS (Global Positioning System) receiver provided spatial reference for the EMI data, which were collected at a rate of several soundings per meter of survey-line length, resulting in more than 5 data points per square meter per hour (20,000 data points per acre per hour). A repeat survey along the entire length of the main runway demonstrated that the inherent variability in apparent electrical conductivity (ECa) measurements, in millisiemens per meter ( $\mathrm{mS} / \mathrm{m})$, was expected to be about $7 \mathrm{mS} / \mathrm{m}$ for EMI surveys in this environment (fig. 9). Thus, a difference of $7 \mathrm{mS} / \mathrm{m}$ is considered to be the minimum threshold for detecting change in playa soil conditions from time-lapse EMI surveying.

\section{Numerical Experiments of Soil Mechanics and the Cracking Process}

Numerical models were used in the present study as an additional tool to test hypotheses and evaluate potential mechanisms related to surface cracking at Bicycle Lake playa. Previous approaches in modeling such processes have relied on continuum mechanics formulations, with solutions obtained by the finite-element method (FEM; for example, Pacheco and others, 2006; Pacheco-Martinez and Arzate-Flores, 2007; Hernandez-Marin and Burbey, 2010; 2012).

Investigating Earth fissures due to groundwater depletion and water-table decline, Pacheco-Martinez and Arzate-Flores (2007) used the FEM to calculate stresses induced by soil consolidation. Water-table decline was modeled by gradually subjecting deeper layers to increased effective stresses, which promoted soil settlement and surface subsidence. The model was representative of a subsurface profile in the Queretaro Valley, Mexico, where differential settlements and regions of stress concentration originated from soil layers of variable thickness and depth. The FEM-based modeling results revealed a close match between zones of computed large horizontal displacement and Earth fissures observed in the field.

The FEM models by Hernandez-Marin and Burbey (2010; 2012) displayed stress concentrations and soil cracking associated with groundwater pumping in the vicinity of geological faults. Ground fissuring was modeled using an enriched finite-element mesh, in an approach known as "extended FEM". Results indicate that stress was localized near the surface expression of the fault and at the interface between the saturated and vadose zones (fig. 5).

Despite their utility, the FEM modelling approaches have some shortcomings arising from their continuum formulation, which are more evident when interface processes are involved. 


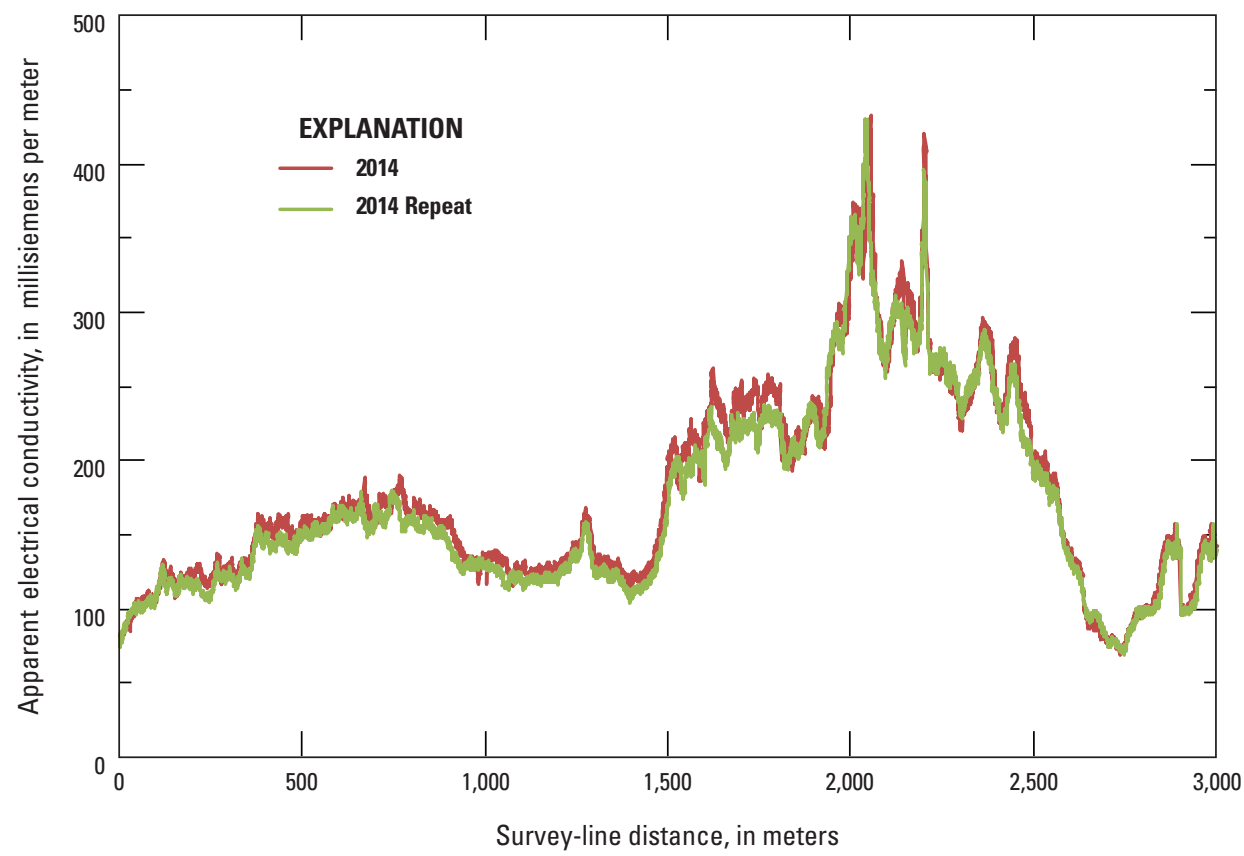

Figure 9. Results from the test of repeatability along a 3-kilometer survey line of the main runway, December 2014, Fort Irwin National Training Center, California, indicating average variability for repeat survey lines is around 7 millisiemens per meter.

In the studies mentioned, elastic constitutive laws represented by an immediate shift in the properties between the saturated and the dry zones were used instead of a gradual transition. This resulted in the unsaturated zone sliding over the saturated zone, which would not be expected in the field, where the soil water-content gradient would prevent such strain localization.

Soil moisture is a key variable in parameterizing mechanical properties of soil. When present in small quantities, water tends to concentrate in bridges between close neighboring grains, where it clings to the grains by surface tensions and generates forces that pull the grains surrounding the pore space toward each other. This state is defined as the pendular regime. As the water content increases, capillary bridges start merging, and the distribution of liquid-phase water is continuous along pore walls, with gaseous phase at the pore center; although some pores can become fully saturated. This state characterizes the funicular regime. Further saturation causes nearly all pore space to be filled with water; however, pore pressure can still be less than atmospheric. Additional increase in water content leads to full saturation, and pore pressures become equivalent to atmospheric or larger (Mitarai and Nori, 2006). This introduces a variety of mechanical responses that require modeling approaches specific to each regime.

One increasingly popular technique in geomechanics for modeling such processes is the discrete element method, originally proposed by Cundall and Strack (1979). For the analysis of the pendular regime, Scholtès and others (2009) and Mani and others (2013) implemented contact-law formulations that extend standard elasto-frictional particle interaction to account for forces associated with the capillary bridges and fluid migration. These applications are relevant to our study of the Bicycle Lake playa.

\section{Expansive Soils and Desiccation Cracking}

The effect of soil moisture on the mechanics of soil deformation is critical, especially in fine-grained soils and sediments that contain expansive clay minerals. When such soils are wetted, particles are hydrated and swell, often to volumes many times larger than the original volume. Conversely, as water evaporates, pore pressures decrease, reaching values much less than atmospheric pressure, promoting matric suction. This causes the effective stress to increase, and the soil skeleton undergoes consolidation. During shrinkage, the matrix becomes denser and stiffer, and the rate of volumetric strain decreases. When the soil becomes stiff enough to prevent further consolidation, the air-water interface penetrates the initially saturated medium. This characterizes the desaturation point and imminence of the soilcracking process (Shin and Santamarina, 2011).

Under unconstrained homogeneous drying, soil can contract freely without developing cracks (Peron and others, 2009). Factors such as soil texture, boundary resistance (frictional or adhesive), and stress concentrations can act as constraints that promote soil cracking, however. Shin and Santamarina (2011) observed that cracks typically start on the surface of defects, where the geometry favors invasion of the air-water interface. This causes an uneven distribution of stresses and creates a crack that is propagated as the air-water interface continues to penetrate the soil. The pores in the vicinity of cracks become more susceptible to the formation of new cracks, which tend to form a polygonal network. For a thorough review of the mechanics of jointing cracks, including desiccation cracks, see Pollard and Aydin (1988). 


\section{Laboratory Analyses for Model Calibration}

In order to assess the shrinkage and cracking potential of the soil at Bicycle Lake playa, laboratory tests were

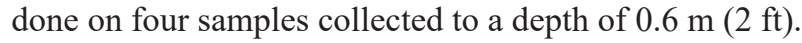
Sieve and hydrometer analyses indicated that the grain-size distribution varied with depth. The sample spanning $0-15 \mathrm{~cm}$ (0-6 in.) depth was composed of 32 percent sand, 36 percent silt and 32 percent clay. The other three samples collected from depths spanning $15-60 \mathrm{~cm}(6 \mathrm{in} .-2 \mathrm{ft})$ were all richer in silt (42-65 percent) and leaner in sand-sized particles (11-16 percent); clay varied from 22 to 43 percent. X-ray diffraction analysis indicated that the clay-sized fraction consisted mostly of smectite with traces of kaolinite. These results indicate that the playa sediments have a high potential for shrink-swell volumetric changes.

A desiccation experiment was carried out that mixed water with an air-dry sample of the playa soil, collected from the 0 to $0.6 \mathrm{~m}$ ( 0 to $2 \mathrm{ft}$ ) depth, until a slurry formed, which was used to fill an aluminum mold measuring 19 by 19 by $4 \mathrm{~cm}$ ( 7.4 by 7.4 by 1.6 in.; fig. 10). The slurry-filled mold was then placed on a digital balance and allowed to dry at room temperature for 100 days, while measurements of mass were recorded every 0.5 seconds by a computer connected to the digital balance. The data provided insight into the temporal change in the gravimetric water content, defined as the ratio of mass of water to mass of solids. Crack aperture was measured using a digital caliper and photographed in select stages of crack development.

Three distinct phases in soil-water content were observed during the experiment. The first phase was characterized by a linear decrease in gravimetric water content from saturation to approximately 17 percent. In this phase, the complete polygonal pattern developed, and the maximum crack aperture was $10 \mathrm{~mm}(0.4 \mathrm{in}$.). An intermediate phase characterized by a reduced rate of drying to about 7 percent soil-water content followed. No additional cracks formed during this stage; existing cracks widened to a maximum aperture of $12 \mathrm{~mm}$ (0.5 in.). In the final phase, soil-water content declined even more slowly until a minimum value of 5 percent was reached; no changes in soil cracks were observed.

Key observations included the first crack opening across the sample at a water content of 33 percent, followed by a full polygon structure forming at a water content of 28 percent (fig. 10). In parallel with these experiments, additional soil samples were analyzed using pressure-plate extractors to determine water-retention curves (soil suction-water content relations). Results of all the laboratory analyses provided the basis for calibration of the discrete element method model used for numerical experiments.
$\boldsymbol{A}$

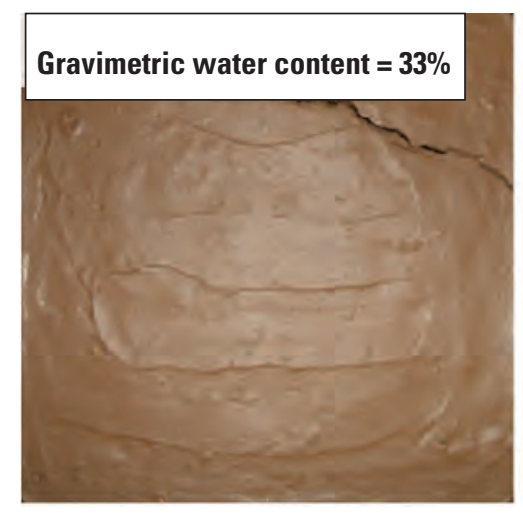

B

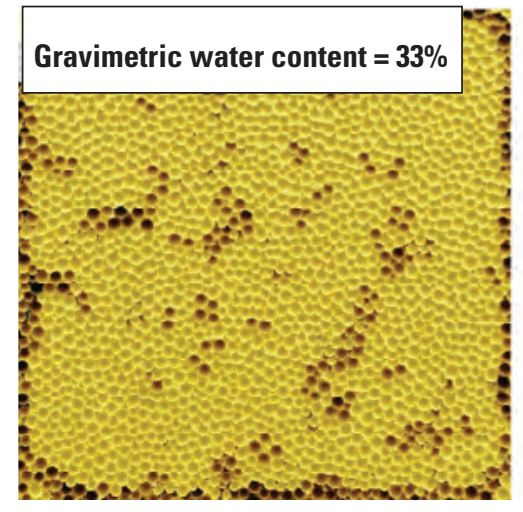

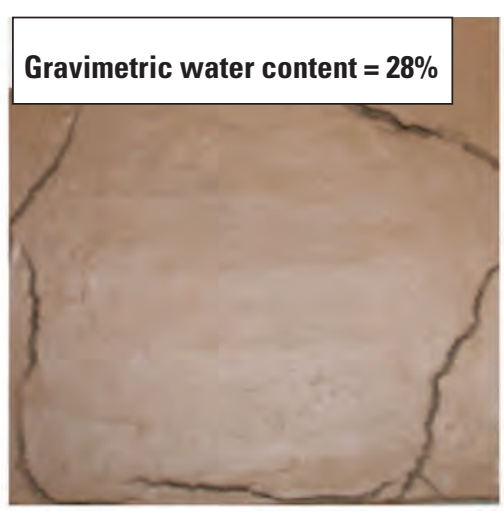

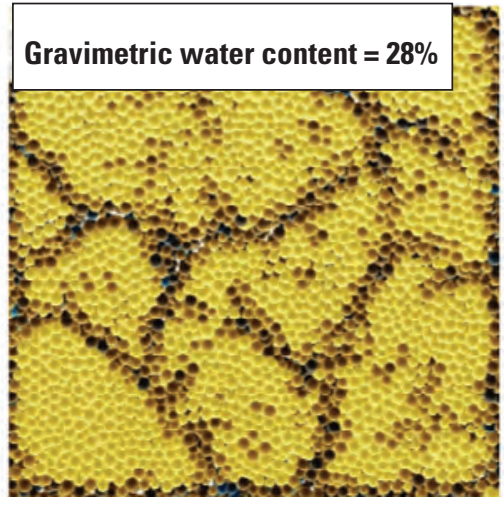

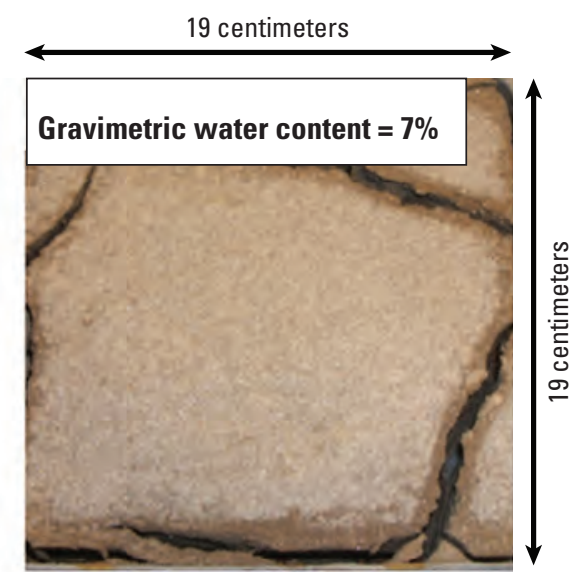

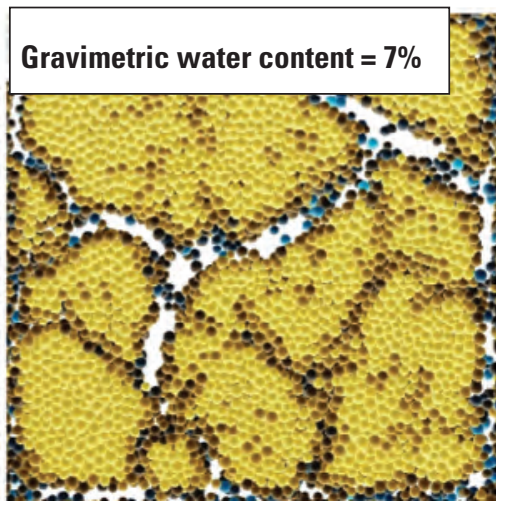

Figure 10. Results of playa soil desiccation according to the gravimetric water content (w), in percent, for discrete element method model parameterization and calibration: $A$, laboratory experiment of soil desiccation through time; and $B$, numerical simulation of the increase in broken bonds between particles as stress builds from volumetric shrinkage due to desiccation. 


\section{Discrete Element Method and Model Calibration}

The discrete element method was chosen as the numerical tool because of its ability to simulate soil-cracking processes. In the discrete element method, the soil domain is discretized as independent spherical elements, the dynamics of which are determined by an explicit time-integration scheme of Newton's second law of motion. Forces resulting from the spatial interaction among elements are computed following a contact model.

The interaction among fine-grained particles in the presence of water is rather complex; it involves adsorption, double-layer attraction, van der Waals, particle interpenetration, and electrical forces. In order to capture this complexity in a straightforward numerical model, the interactions among particles were defined by bonded contacts, and properties were parametrized as a function of the gravimetric water content. The contact law used in this particular problem was established by Scholtès and Donzè (2012) and implemented in the open-source software Yade-discrete element method (Šmilauer and others, 2015). The force, $F$, at iteration $i$, in the normal direction $n$, in compression and extension is expressed as follows:

$$
\begin{gathered}
F_{n}^{i}=k(w)_{n} \Delta D \\
F_{n}^{\max }=r(w)_{n}
\end{gathered}
$$

where

$k(w)_{n} \quad$ is the normal stiffness (Newtons per meter) as a function of the gravimetric water content, $w$, as a percentage, and

$\Delta D \quad$ is the relative displacement between two elements in relation to the original equilibrium distance $(\Delta D=D-D e q)$.

Under tension, if the normal force (eq. 5) overcomes the tensile strength $\left(r(w)_{n}\right)$, equivalent to the maximum normal force $\left(F_{n}^{\max }\right)$ at a given water content (eq. 6), the bond is broken, and the normal force is reset to zero at the subsequent temporal iteration. This is comparable to a failure in the continuum fabric. Shear contact forces are calculated in an analogous fashion (see Scholtès and Donzè, 2012, for details). In this study, the bond stiffness and strength were assumed to have the same characteristics for the normal and tangential components.

Various authors have proposed empirical relationships to describe the soil drying-shrinkage process at the element scale and the development of the contact model properties (stiffness and tensile strength) as functions of water content (Kodikara and Choi, 2006; Peron and other, 2009; Amarasiri and others, 2011; Sima and others, 2014). These served as the basis for the relationships in this study, for which input parameters were calibrated according to the water-retention curve and desiccation-cracking experiments on playa sediment samples.

Model calibration consisted of fitting the equations for soil stiffness and tensile strength to provide a good match to the observed progression of crack development in the laboratory desiccation experiment (fig. 10). Equations 7 and 8 define the evolving stiffness, $k$, and tensile strength, $r$, as functions of the gravimetric water content, $w$, post calibration. The free parameters adjusted in the calibration correspond to the linear coefficients in both equations:

$$
\begin{gathered}
k(w)_{n}=1.63 \times 10^{8} \exp (w) \\
r(w)_{n}=9.81-3.46 \times 10^{(-8)} \exp [\exp (w)]
\end{gathered}
$$

In order to simulate the overall volumetric reduction experienced by the desiccating samples, element diameters shrank according to equation 9. There was a direct correlation between simulated and real time, with time-steps equivalent to 1 second. The water-content relationship to time (Sima and others, 2014) is represented by equation 10 . The process unfolded in quasi-static conditions, reflecting the process observed in the laboratory.

$$
d(w)=d_{0}\left[1-\exp \left(a \frac{w-w_{0}}{w_{f}-w_{0}}\right)\right]
$$

$$
w=\frac{t}{t_{f}}\left(w_{f}-w_{0}\right)+w_{0}
$$

where

$$
\begin{aligned}
d(w) & \text { is the element diameter [L]; } \\
d_{0} & \text { is the initial diameter [L]; } \\
a & \text { is the shrinkage coefficient [dimensionless], } \\
& \text { which ranged from } 0.1134 \text { to } 0.0859 \\
& \text { according to the decay in water content as } \\
& \text { a function of time (eq. 10); } \\
w_{0} & \text { is the initial water content [percent]; } \\
w & \text { is the current water content [percent]; } \\
w_{f} & \text { is the final water content [percent]; } \\
t & \text { is the current time [s]; and } \\
t_{f} & \text { is the final time [s]. }
\end{aligned}
$$

Calibration was carried out by adjusting the free parameters iteratively, so as to closely reproduce the characteristic size of polygons formed in the desiccationcrack experiments. Initially, a confined region (fixed, rigid boundary planes) of the same dimensions as in the laboratory experiments was populated with approximately 40,000 spherical elements, following the procedure proposed 
by Dang and Meguid (2010), at a uniform size distribution with a coefficient of variation of 30 percent. Subsequently, a layer of two elements at the lateral boundaries and the bottom of the domain had all degrees of freedom blocked to represent the mold of the laboratory desiccation experiments. Hence, the model had zero-displacement/rotation boundaries on the horizontal extremities as well as on the lower vertical plane. The upper part of the domain had a free surface, no stress/ displacement boundary. A layer of three elements above the fixed bottom was used to represent the interface between the sample and the laboratory mold.

Once the model properties were calibrated, these were used to evaluate hypotheses concerning playa cracking patterns. The first set of numerical simulations was aimed at evaluating the hypothesis, first put forward by Neal and others (1968), that giant desiccation cracks in macropolygon morphology form on playas as a result of water-table decline and the associated desiccation of the overlying capillary-fringe zone (figs. 6A, $B$ ). To simulate these conditions, the model was extended to incorporate varying amounts of water-table decline to determine if shrinkage stresses could propagate upward through the overlying dry soil and cause cracking up to the ground surface (fig. 11).

A second set of numerical experiments was motivated because although a varying water table in shrink-swell soils in semi-arid to arid climates is not entirely unusual, reports of macropolygons from giant desiccation cracks appear to be isolated to the southwest part of the United States and northern Mexico. These features all seem to be in the Basin and Range Province, which is a region of active extensional tectonics. Thus, an alternative hypothesis is proposed in which soil desiccation in the presence of a regional tensile stress field could be the key requirement needed for the genesis of macropolygons that have giant desiccation cracks. Two key questions addressed by the numerical model in this suite of simulations (fig. 12) were whether tectonic stresses can be shown to affect desiccation-driven cracking patterns and whether these coupled processes (extensional tectonics and desiccation) might explain the propagation of large cracks upward to the ground surface.

\section{Layer subject to hydrologic cycle}

\section{Layer in quasi-equilibrium (no initial stresses)}

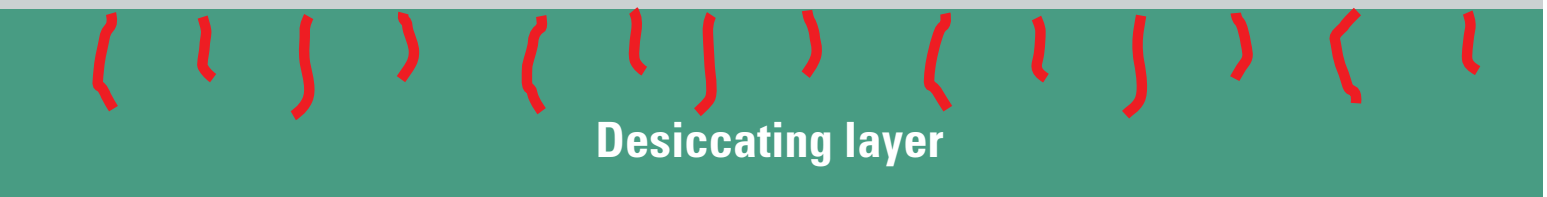

Figure 11. Numerical experiment to evaluate the conventional hypothesis of macropolygon formation as giant desiccation cracks from a declining water table at depth.

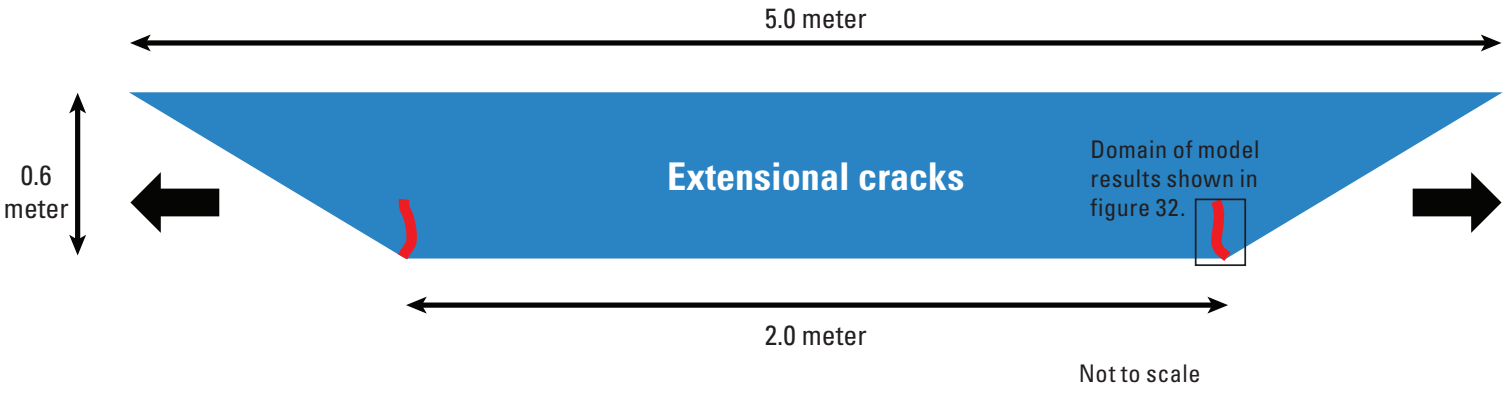

Figure 12. Numerical experiment to evaluate the potential role of regional tectonic stress in propagating extensional cracks upward to the land surface in conjunction with the desiccation process. 


\section{Results}

\section{Water Levels}

Since pumping began in Bicycle Basin during the mid1960s, water levels have declined in response to groundwater withdrawals (figs. $13 A-C$; locations of wells shown in fig. 3 ). In the northwestern part of the basin, water levels declined about $30 \mathrm{~m}(100 \mathrm{ft})$ in wells 14N/3E-13M1S and -13M4 (fig. 13A). These wells are north of the South Coyote Canyon fault. In the central part of the basin (fig. 13B), water levels declined about 23-27 m (75-90 ft) in wells 14N/3E-14P2 and $14 \mathrm{~N} / 3 \mathrm{E}-23 \mathrm{~B} 1-3$ in the west-central part and in the area of subsidence (fig. 13B) and in wells 14N/3E-13K1 and 14N/4E$18 \mathrm{~N} 2$ in the east-central part (fig. 13C).

Wells 14N/3E-23B1-3 make up a multiple-completion site in the center of an area of subsidence (fig. 3). Water levels in $14 \mathrm{~N} / 3 \mathrm{E}-23 \mathrm{~B} 2$, perforated in the main production zone in the Quaternary-Tertiary older lacustrine $(Q T o l)$ deposits of the upper aquifer, mimicked water levels measured in well $14 \mathrm{~N} / 3 \mathrm{E}-14 \mathrm{P} 2$, next to the pumping well 14N/3E-14P1. Water levels in the deeper well 14N/3E-23B1, perforated in Tertiary younger gravels (Tyg), reflected seasonal changes, but were damped slightly, indicating that less water was supplied to the pumping well from the lower aquifer and Tertiary younger gravels than from the upper aquifer. Water levels in the shallowest well 14N/3E-23B3 showed slight seasonal changes and represented the water table. Water levels in well 14N/3E$23 \mathrm{G} 1$, a test hole about $0.6 \mathrm{mi}$ from $14 \mathrm{~N} / 3 \mathrm{E}-14 \mathrm{P} 2$ and about $2.4 \mathrm{~km}(1.5 \mathrm{mi})$ from $14 \mathrm{~N} / 4 \mathrm{E}-18 \mathrm{~N} 2$, showed minimal seasonal change and a similar trend as for well 14N/3E-23B3. The water-surface altitude in 14N/3E-23G1 was about $9 \mathrm{~m}(30 \mathrm{ft})$ higher than those in the $14 \mathrm{~N} / 3 \mathrm{E}-14 \mathrm{P} 2$ and $14 \mathrm{~N} / 3 \mathrm{E}-23 \mathrm{~B}$ wells and was similar to the altitude in wells $14 \mathrm{~N} / 3 \mathrm{E}-24 \mathrm{Q}$ on the east side of the basin. Two possible reasons for this altitude difference are (1) the lower perforations of 14N/3E-23G1 were plugged, which long-term changes in water-quality samples in this well support (Densmore and others, 2018), or (2) a groundwater barrier could exist between 14N/3E-23G1 and $14 \mathrm{~N} / 3 \mathrm{E}-14 \mathrm{P} 2$, although no barrier has been mapped in this part of the basin. Additional testing of the groundwater-barrier hypothesis in the groundwater-flow and subsidence model showed the model was insensitive to a fault in this location. In the southern part of the basin, little or no water-level decline was measured for wells 14N/3E-22N1, -22P1, -27E3, and -28A1 (fig. 13D), indicating that these wells are separated from the pumping by faults, differences in lithology, or other horizontal-flow barriers.

A 2017 map of water-table contours, created from measurements in the shallowest well in a multiple-well monitoring site, and in the productions wells, is shown in figure 14. Water levels were lowest in the central and northeastern part of the basin as a result of pumping, primarily from wells 14N/3E-14P1 and 14N/4E-18N1.

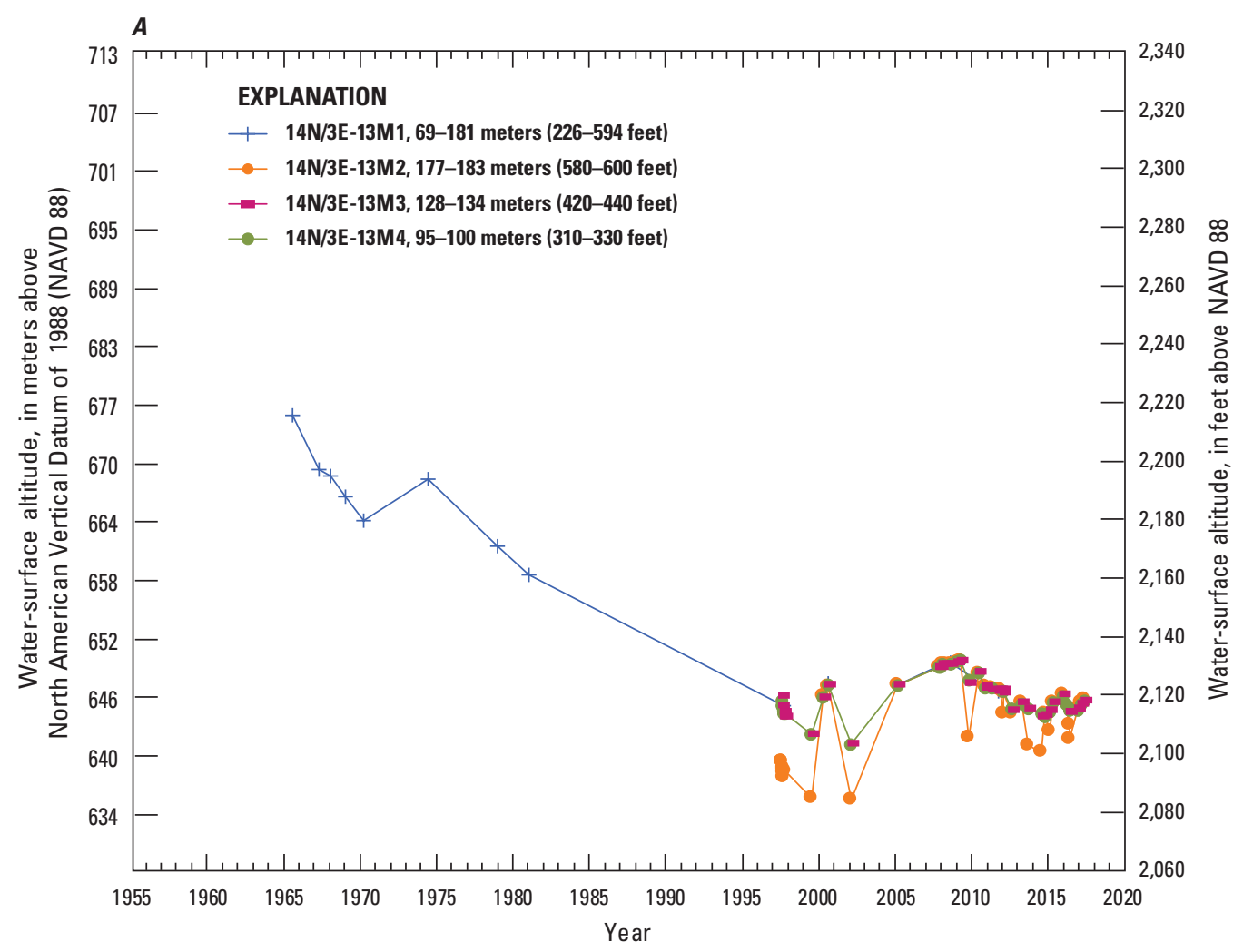

Figure 13. Water-level changes in selected wells during 1955-2017 in Bicycle Basin, Fort Irwin National Training Center, California, for the following areas: $A$, northern; $B$, central (west); $C$, central (east); and $D$, southern. 

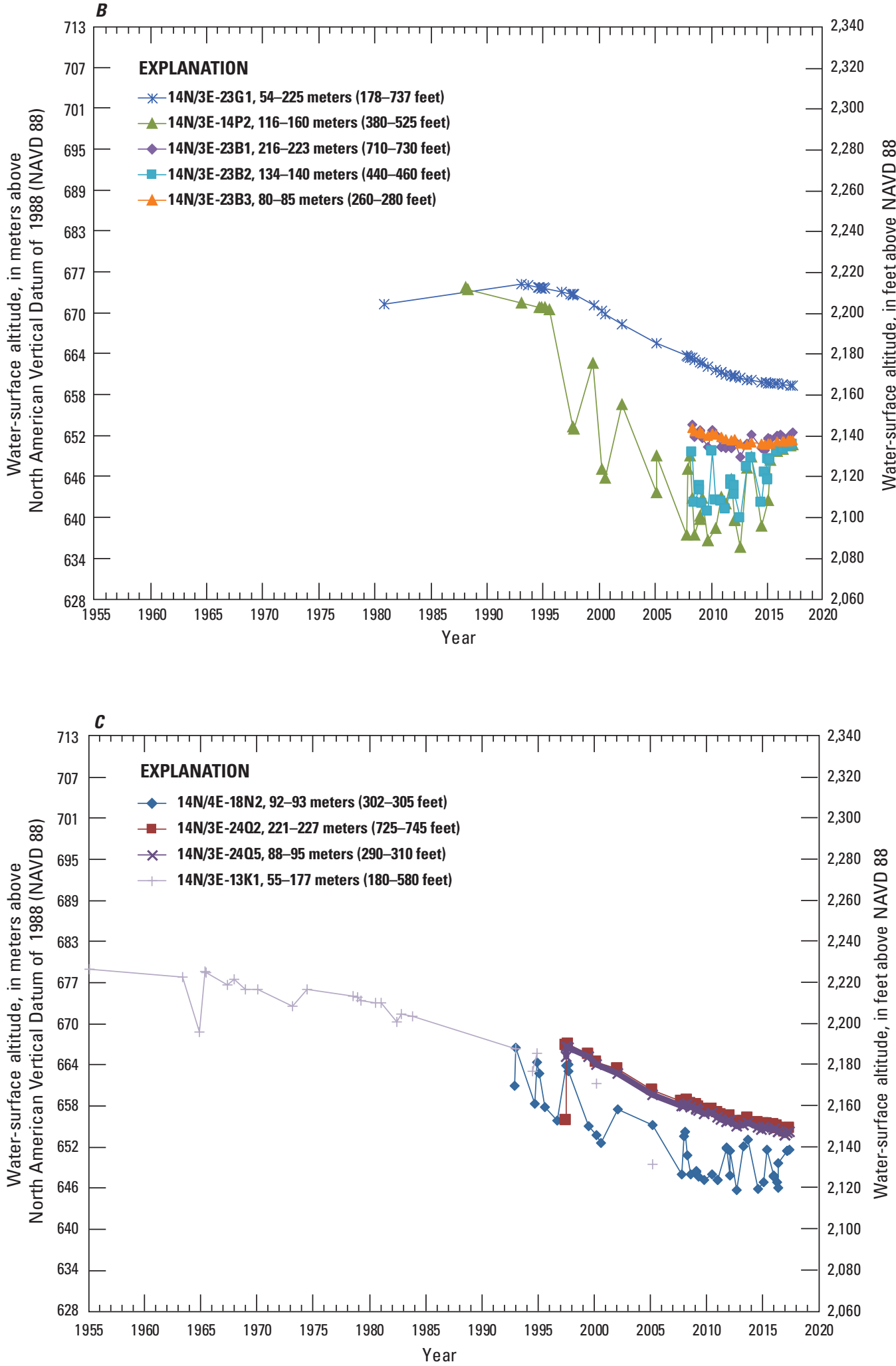

Figure 13. - Continued 


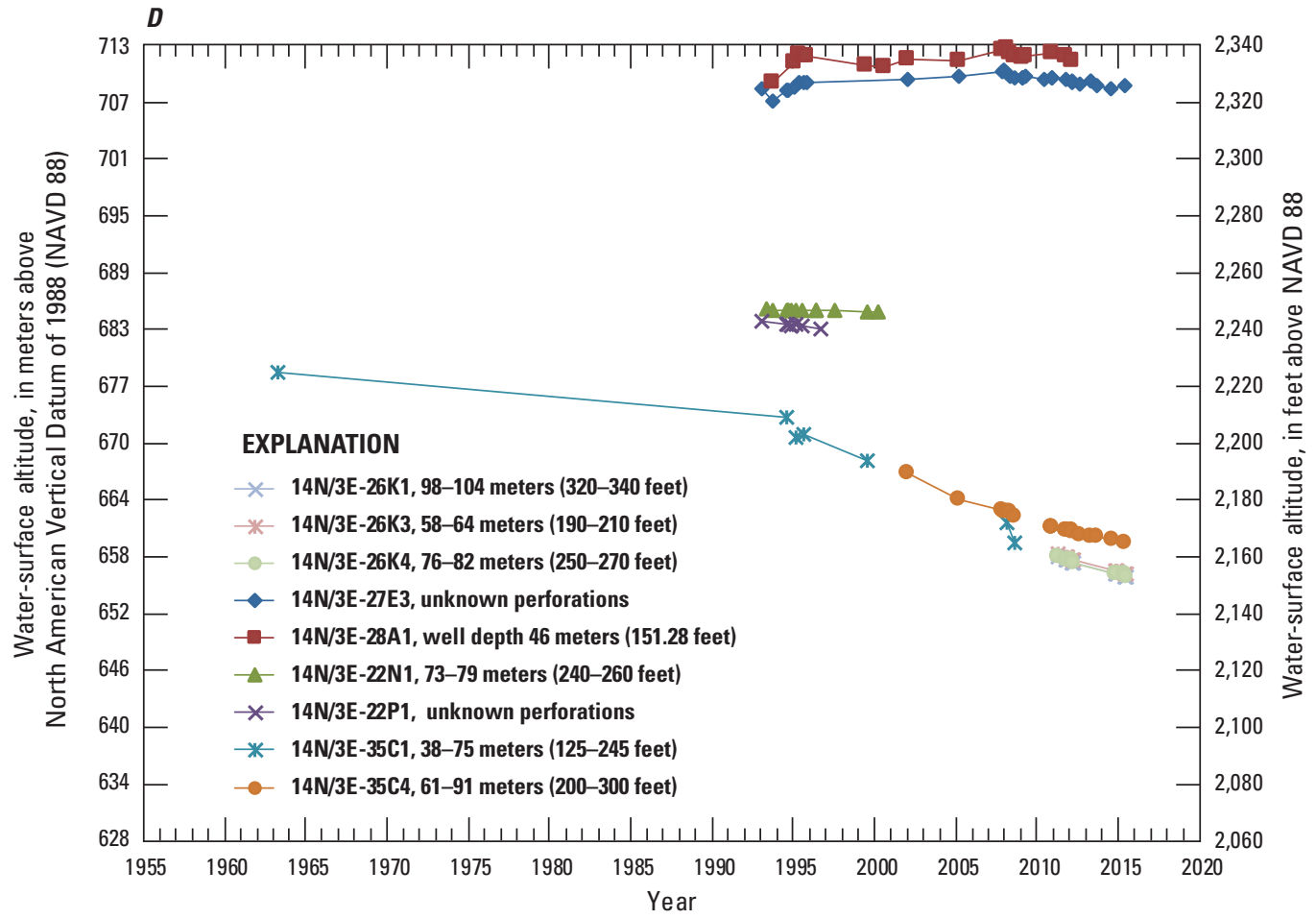

Figure 13. - Continued

Continuous water levels showed seasonal changes in wells $14 \mathrm{~N} / 3 \mathrm{E}-14 \mathrm{P} 2$ and $-23 \mathrm{~B} 2$ related to changes in groundwater withdrawal (figs. $15 A, B$ ). Water-level declines began in the early 1990 s in well $14 \mathrm{~N} / 3 \mathrm{E}-14 \mathrm{P} 2$ shortly after pumping began in the late 1980s. Overall, water levels continued to decline between December 2007 and June 2015. During this time, water levels partly recovered during periods of decreased pumping, which is generally during the fall or winter. Pumping did not stop for long enough, however, to allow water levels to recover to previous water levels (figs. 15B,C). Although the overall trend in water levels during this time was generally downward, water levels in the main pumping zone, primarily in Quaternary-Tertiary older alluvium (QToa), showed recovery that correlated with a reduction in pumping beginning during 2013 and 2015.

Water-level data from multiple-well monitoring sites indicate that the water level varied by depth in some areas (fig. 15B). In the area of maximum subsidence, water levels differed greatly at multiple-well monitoring site $14 \mathrm{~N} / 3 \mathrm{E}-$ 23B1-3, specifically between the middle well 14N/3E-23B2, perforated from 134 to $140 \mathrm{~m}(440-460 \mathrm{ft})$ in the main pumping zone in Quaternary-Tertiary older alluvium (QToal) of the upper aquifer, and the deep and shallow wells, 14N/3E$23 \mathrm{~B} 1$ and 3, respectively. The shallow well is perforated in Quaternary-Tertiary older alluvium (QToa2) of the upper aquifer, and the deep well is perforated in the Tertiary younger gravels (Tyg) of the lower aquifer. Water levels in the deep and shallow wells were both higher than water levels in the middle well, indicating upward, vertical flow from the deep well and downward flow from the shallow well. Based on this difference in water level, the Quaternary-Tertiary older alluvium was subdivided into two parts (QToa1 and QToa2) during groundwater-flow modeling calibration (Densmore and others, 2018). Quaternary-Tertiary older alluvium (QToal) of the upper aquifer represents the main pumping zone in this area.

\section{Land Surveys}

Baseline geodetic leveling, EDM, and TLS surveys were carried out in January 2009. A baseline 3-D image was generated from the TLS data of the fissure and surrounding area. A repeat EDM survey completed in October 2009 was inconclusive, possibly indicating more time was needed to monitor horizontal changes; however, upon further assessment, the EDM surveys were discontinued because of the lack of detectable horizontal change along the transect using this method. Repeat leveling and TLS surveys were done in September 2010, April 2011, October 2014, November 2015, and December 2016 to assess changes related to groundwater withdrawals through time. 


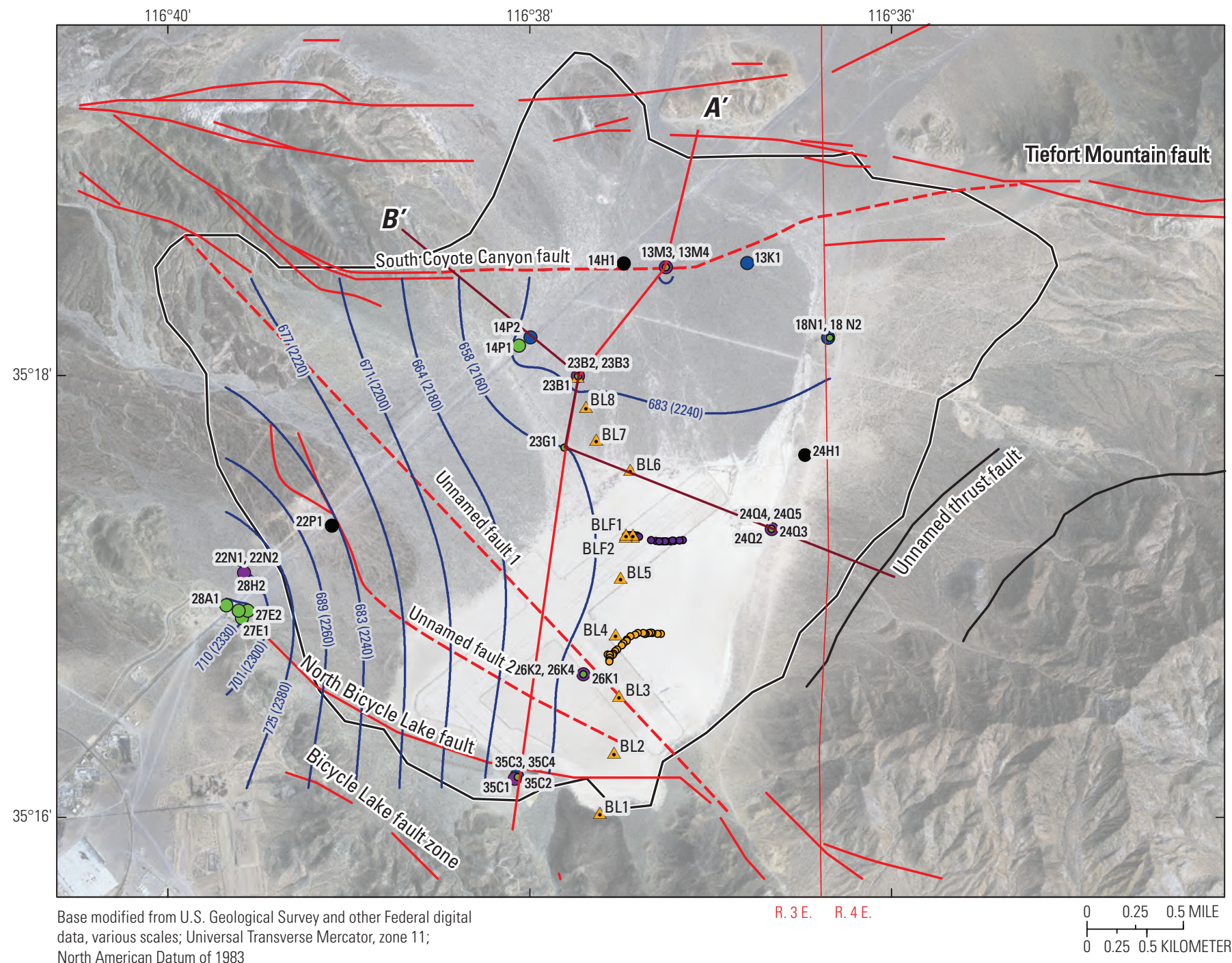

North American Datum of 1983

EXPLANATION

Water-table contours were approximated from water levels measured during 2017

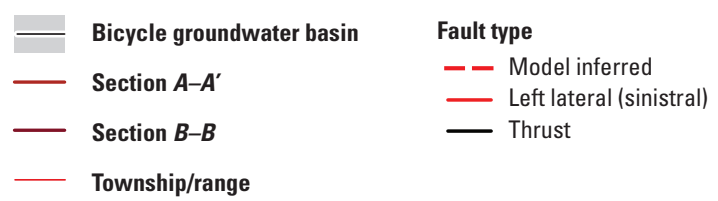
- Monuments
- Crack 2013
- Crack 2005-06
Water-level contour,
in meters ( $\mathrm{ft}$ )

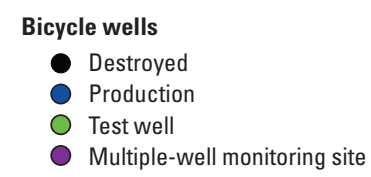

Figure 14. Water-table contours approximated from water levels measured during 2017 in Bicycle Basin, Fort Irwin National Training Center, California.

\section{Vertical Land-Surface Change Along Leveling Transect}

The time-series leveling data (fig. 16) tracked the vertical land-surface changes between monuments along the leveling transect (fig. 7). The leveling data were particularly helpful for determining land-surface deformation on the playa surface, where InSAR data were ineffective because of decorrelation of the ground-reflector data likely caused by clays on the playa that swelled and dried following infrequent flooding and lake-like conditions or grain movement from wind.
Land-surface changes were calculated along the transect for five periods (January 2009 to October 2010, October 2010 to April 2011, April 2011 to October 2014, October 2014 to November 2015, and November 2015 to December 2016). To determine the change relative to stable bedrock (benchmark BL1), the elevation difference was calculated by subtracting the 2009 baseline elevation at each benchmark from the elevation at each benchmark for each survey year. To determine the vertical land-surface change for any given year, the elevation difference between benchmark pairs along the transect was summed and are relative to the stable bedrock benchmark (BL1). 

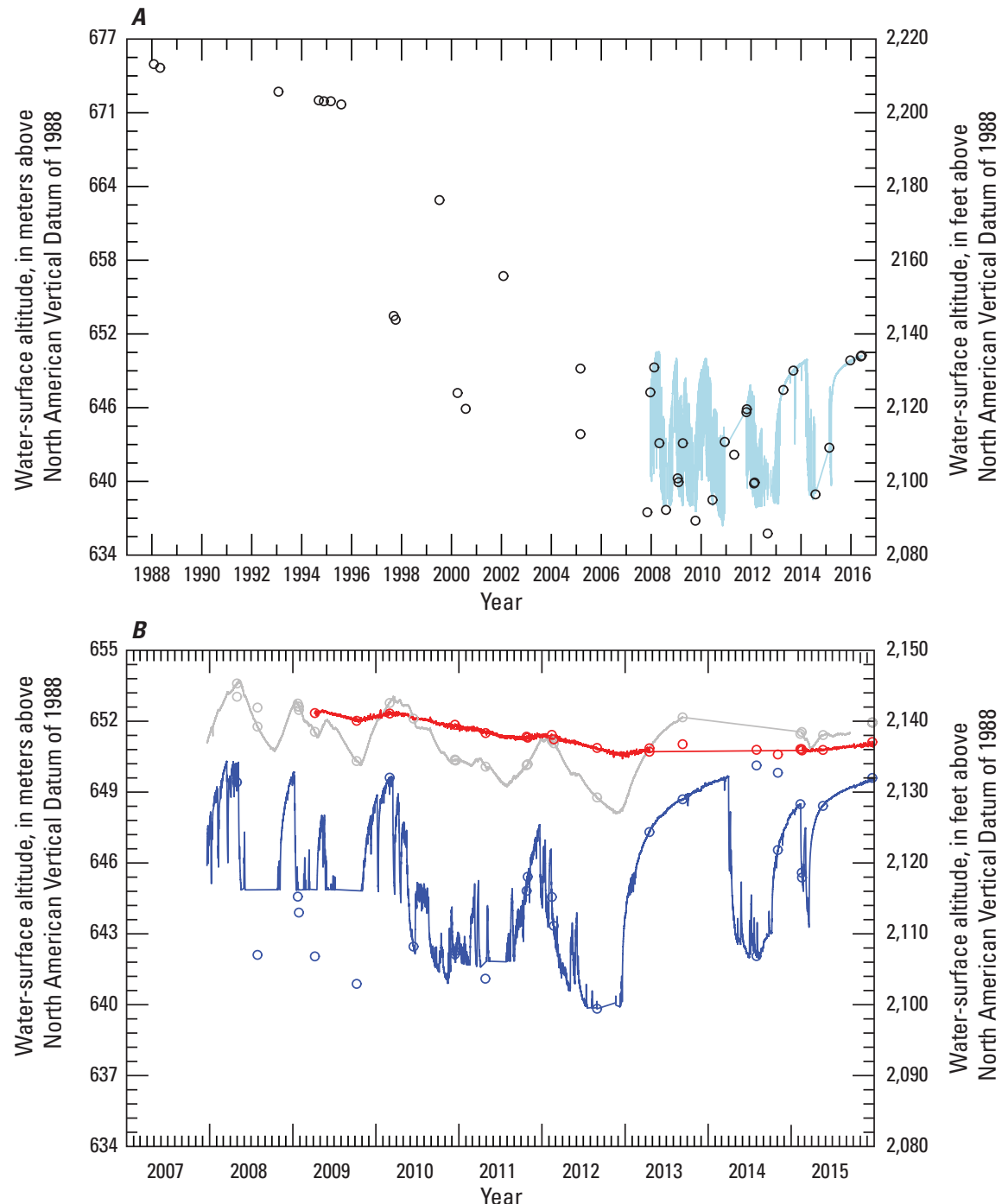

\section{EXPLANATION \\ Continuous water levels, 14N/3E-14P2 \\ - Manual water levels}

\section{EXPLANATION}

Continuous water levels

$-14 \mathrm{~N} / 3 \mathrm{E}-23 \mathrm{~B} 1$

$-14 \mathrm{~N} / 3 \mathrm{E}-23 \mathrm{~B} 2$

$-14 \mathrm{~N} / 3 \mathrm{E}-23 \mathrm{~B} 3$

Manual water levels

$14 \mathrm{~N} / 3 \mathrm{E}-23 \mathrm{~B} 1$

- $14 \mathrm{~N} / 3 \mathrm{E}-23 \mathrm{~B} 2$

- $14 \mathrm{~N} / 3 \mathrm{E}-23 \mathrm{~B} 3$

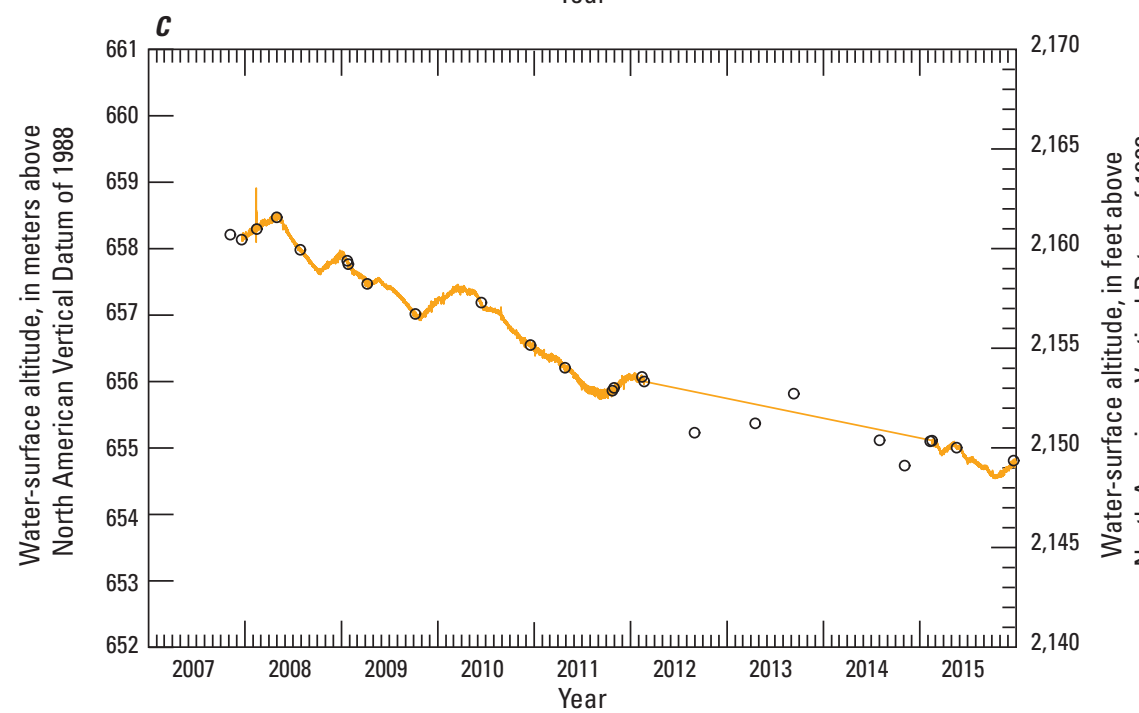

\section{EXPLANATION}

- Continuous water levels, 14N/3E-2405

- Manual water levels

Figure 15. Continuous water-level changes, Bicycle Basin, Fort Irwin National Training Center, California: $A$, well 14N/3E-14P2 near area of subsidence during 1988-2015; $B$, well 14N/3E-23B1-3 near area of subsidence during 2007-15, and $C, 14 \mathrm{~N} / 3 \mathrm{E}-2405$ east of the area of subsidence during 2007-15. 


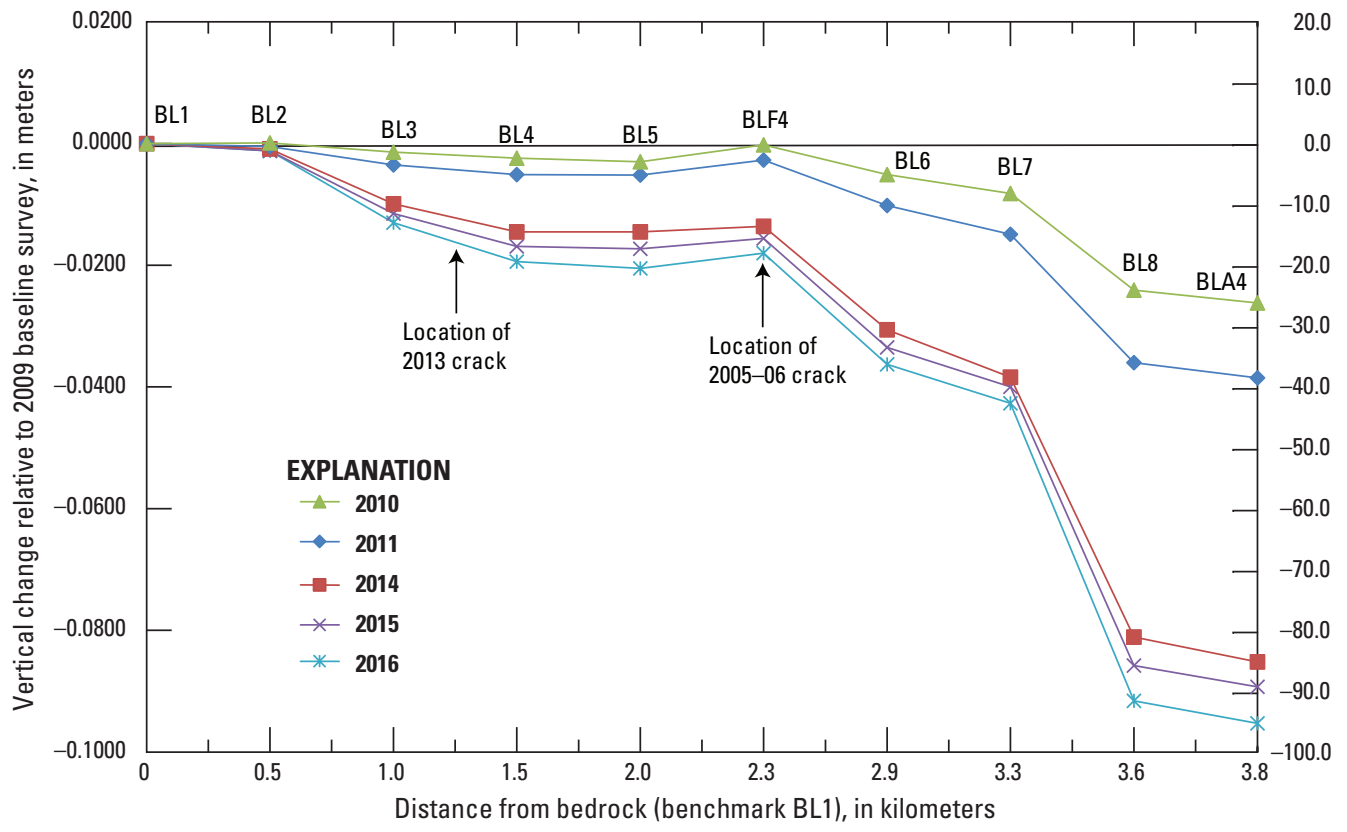

Figure 16. Vertical change along transect for repeat leveling surveys relative to 2009 baseline survey in Bicycle Basin, Fort Irwin National Training Center, California (location of transect is shown on fig. 7).

Leveling data showed differential subsidence along the transect between 2009 and 2016 (fig. 16), with less subsidence south of the 2005-06 crack between BL2 and BLF4, more subsidence north of the 2005-06 crack between BLF4 and BL7, and the largest subsidence gradient from BL7 to BL8 before flattening out between BL8 to BLA4. South of the 2005-06 crack, little vertical change was measured between monuments BL1 and BL2; however, about $3 \mathrm{~mm}$ of subsidence was measured at monuments BL3, BL4, and BL5 during 2009-10. By 2016, more than $13 \mathrm{~mm}$ of subsidence was measured at monument BL3, and about $20 \mathrm{~mm}$ was measured at monuments BL4 and BL5. Note that the 2013 crack is between monuments BL3 and BL4 (fig. 7). North of the 2005-06 crack, 5-8 mm of subsidence was measured at BL6 and BL7, and more than $24 \mathrm{~mm}$ was measured at BL8 and BLA4 during 2009-10; by 2016, 36 to about $43 \mathrm{~mm}$ of subsidence was measured at BL6 and BL7, and about $90-95 \mathrm{~mm}$ was measured at BL8 and BLA4, respectively.

Incremental subsidence at BLA4 based on measurements at the benchmarks along the entire transect was $26 \mathrm{~mm}$ (1 in.) from January 2009 to September 2010, $12 \mathrm{~mm}$ (0.5 in.) from September 2010 to April 2011, and $47 \mathrm{~mm}$ (1.85 in.) from April 2011 to October 2014. The data show that the subsidence rate was similar between 2009 and 2014: $1.3 \mathrm{~mm} / \mathrm{month}$ (mo; $0.05 \mathrm{in} . / \mathrm{mo}$ ) from January 2009 to September 2010, $1.7 \mathrm{~mm} / \mathrm{mo}(0.07 \mathrm{in} . / \mathrm{mo})$ from September 2010 to April 2011, and $1.1 \mathrm{~mm} / \mathrm{mo}$ (0.04 in./mo) from April 2011 to October 2014. The incremental subsidence from October 2014 to November 2015 and November 2015 to December 2016 was only $4 \mathrm{~mm}$ (0.16 in.) and $6 \mathrm{~mm}(0.23 \mathrm{in})$, respectively. The data show that the subsidence rate slowed to $0.3 \mathrm{~mm} / \mathrm{mo}(0.01 \mathrm{in} . / \mathrm{mo})$ from
October 2014 to November 2015 and to $0.5 \mathrm{~mm} / \mathrm{mo}$ (0.018 in./mo) from November 2015 to December 2016 in response to the decrease in pumping that began in 2013, the increase during 2014, and a decrease again in 2015-16.

\section{Ground Movement Across the 2005-06 Main- Runway Crack}

Tapex measurements were collected in January 2009 and, beginning in March 2009, were collected sporadically by Fort Irwin personnel (fig. 17). Repeat tapex measurements from April 2009 to November 2017 indicated opening of the crack between BLF3 and BLF4 (fig. 17B), but no significant change between BLF1 and BLF2 at the western terminus of the crack (fig. 17A). The data collected from 2009 through 2011 exhibited an apparent uncertainty range of about plus or minus $1 \mathrm{~mm}$, whereas data collected from 2014 through 2017 showed greater variability between measurements. Although all data were corrected for temperature, it appears that temperature effects could have adversely affected some readings, and measurements collected during the morning were presumed to be more stable and repeatable. Based on a best-fit linear regression, the overall trend of the data between BLF3 and BLF4 indicated that the crack opened approximately $13.7 \mathrm{~mm}(0.5 \mathrm{in}$.) between April 2009 and

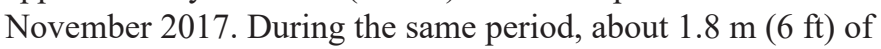

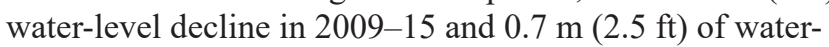
level rise in 2015-17 were measured in the main pumped zone at monitoring site BLA4 (in the area of greatest measured subsidence). During 2013 and 2015-16, pumping decreased in Bicycle Basin (Chris Woodruff, Fort Irwin NTC, written commun., 2017). 

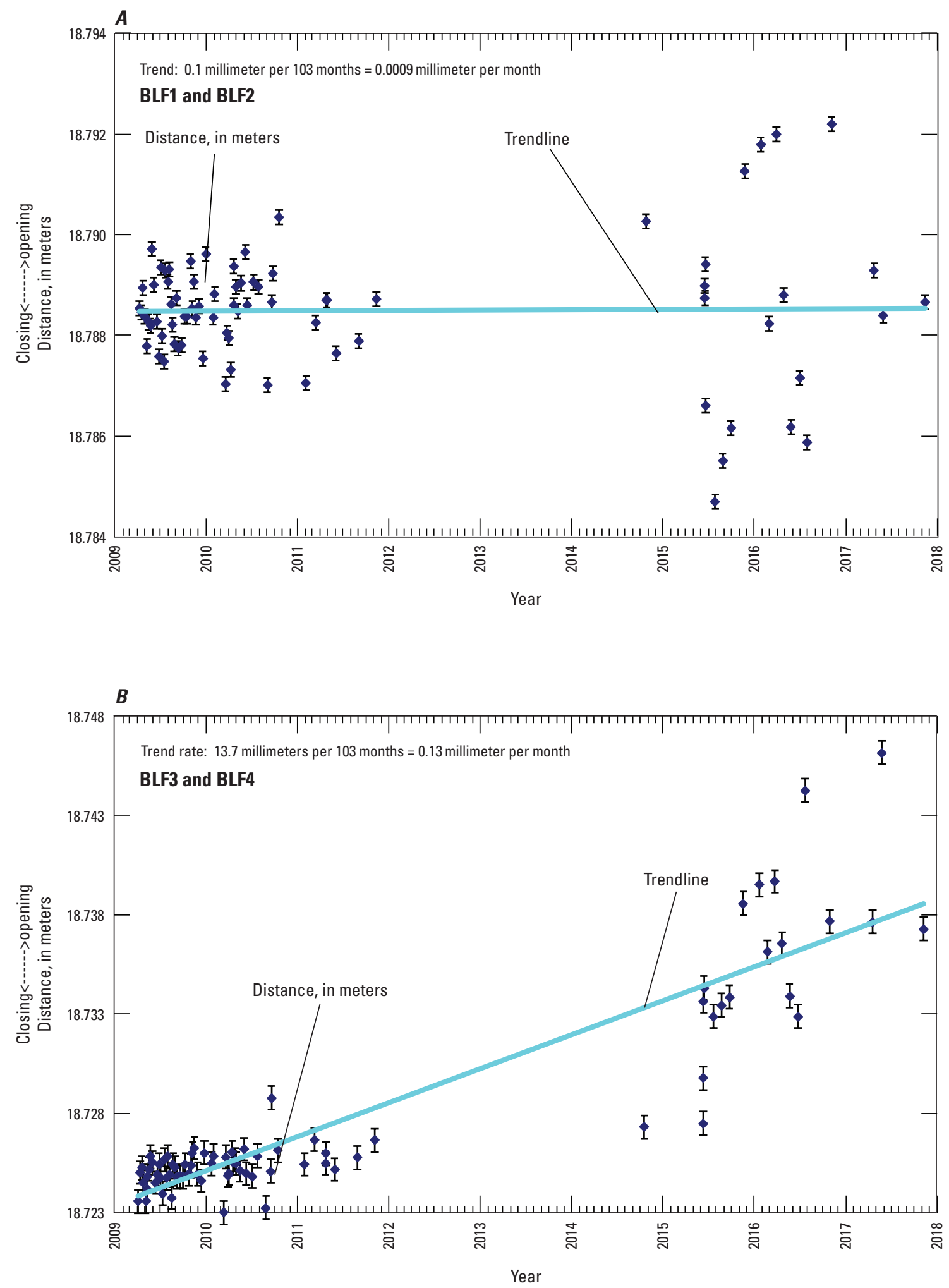

Figure 17. Change in distance between monuments in Bicycle Basin, Fort Irwin National Training Center, California (locations of monuments are shown on fig. 7): $A, B L F 1$ and BLF2; and $B, B L F 3$ and BLF4. 
The baseline lidar survey of the 2005-06 crack was done in January 2009 to track extension across the crack using the four monuments on the playa (BLF1-4; fig. 7). The high-resolution lidar data were also used to track vertical land-surface change (fig. 18) by comparing (differencing) sequential surveys (that is, 2009-10, 2010-11, 2011-14, 2014-15, 2015-16). A total change image from 2009 to 2016 was also created from a comparison of the 2009 survey data with the 2016 survey data.

During the 20 months from January 2009 to September 2010 (fig. 18A), the edges of the crack in the western part of the scanned area deepened 8 plus or minus $2 \mathrm{~cm}$ (3.1 plus or minus 0.8 in.), likely due to erosion from precipitation runoff events. Historical precipitation records from Barstow show total precipitation was $11.4 \mathrm{~cm}$ (4.5 in.) in 2008, $4 \mathrm{~cm}$ (1.6 in.) in 2009, and $39.6 \mathrm{~cm}$ (15.59 in.) in 2010 (National Oceanic and Atmospheric Administration, http://www.ncdc.noaa.gov/cdo-web/datasets/ANNUAL/ stations/COOP:040521/detail; accessed September 4, 2015). Standing water was observed in the crack in January 2009 during the baseline scan and in September 2010 , both following a month when about $2.5 \mathrm{~cm}$ (1 in.) of precipitation fell. Maximum subsidence in the crack along the eastern edge of the scanned area was estimated to be $0.18 \mathrm{~m}$ (7 in.).

Subsequent surveys were compared with previous surveys to determine vertical change through time (figs. 18B-D). During the 7 months from September 2010 to April 2011 (fig. 18B), $0.75 \mathrm{~m}$ (29.5 in.) of subsidence was observed in the crack along the western end of the scanned area. Near the center of the scanned area, part of the crack filled in about $0.14 \mathrm{~m}$ (5.5 in.), likely as a result of inundation of the playa after storms during December 2010. During December 2010, $18 \mathrm{~cm}$ (7.07 in.) of precipitation fell at Barstow (National Oceanic and Atmospheric Administration, http://www.ncdc.noaa.gov/cdo-web/datasets/ANNUAL/ stations/COOP:040521/detail; accessed September 9, 2015), and $3 \mathrm{~cm}$ (1.24 in.) fell at Bicycle Lake Airfield (Wunderground, https://www.wunderground.com/history/ airport/KBYS/2010/12/1/MonthlyHistory.html?req city $=\&$ req $\_$state $=\&$ req $\_$statename $=\&$ reqdb.zip $=\&$ reqdb. magic $=\&$ reqdb.wmo $=;$ accessed April 12, 2016). Standing water was not present in the western part of the crack during April 2011. Additionally, erosion of the playa surface was observed along the eastern end of the crack in the scanned area where the berm had been breached, presumably during the December storms. Approximately $2-3 \mathrm{~cm}(0.8-1.8 \mathrm{in}$.) had eroded from the playa surface along the eastern edge of the scanned area.

During the 42 months from April 2011 to October 2014 (fig. 18C), $0.8 \mathrm{~m}$ (31.5 in.) of subsidence was observed in the crack along the western end of the scanned area, resulting in a pit about $0.9 \mathrm{~m}$ (35 in.) deep. Near the center of the scanned area, about $2-3 \mathrm{~cm}(0.8-1.8 \mathrm{in}$.) of sediment had eroded from the playa surface. Precipitation records for 2011-15 show precipitation ranged from 6 to $8.5 \mathrm{~cm}$ (2.4 to $3.35 \mathrm{in}$.) at Barstow during 2012 and 2011, respectively (National Oceanic and Atmospheric Administration, http://www.ncdc.noaa.gov/ cdo-web/datasets/ANNUAL/stations/COOP:040521/detail; accessed September 4, 2015) and from 1.8 to $8.4 \mathrm{~cm}(0.7$ to 3.3 in.) at Bicycle Lake Airfield during 2011 and 2015, respectively (Wunderground, https://www.wunderground.com/ history/airport/KBYS/2011/4/1/MonthlyHistory.html?r; accessed April 12, 2016). The subsidence pit observed in the scanned area likely resulted from erosion during heavy rain on August 25, 2013, when $4.7 \mathrm{~cm}$ (1.86 in.) of rain fell in a short time, resulting in flooding on the base.

During the 13 months from October 2014November 2015 (fig. 18D), no vertical change was observed in the playa surface. Standing water in the crack and minor erosion on the playa surface at the crack were observed near the eastern end of the scanned area, probably from a storm in October 2015, during which $4.8 \mathrm{~cm}$ (1.9 in.) of rain was recorded at Bicycle Lake Airfield (Wunderground, https://www.wunderground.com/history/airport/ KBYS/2015/10/1/MonthlyHistory.html?req_city=\&req state $=\&$ req $\_$statename $=\&$ reqdb.zip $=\&$ reqdb. magic $=\&$ reqdb. wmo $=$; accessed April 12, 2016).

During the 13 months from November 2015 to December 2016 (fig. 18E), $0.2 \mathrm{~m}$ (7.9 in.) of subsidence was observed in the crack along the western end of the scanned area; standing water was also observed on this part of the crack during this period. Little to no change was observed in the crack in most of the scanned area.

During the 95 months from January 2009 to December 2016 (fig. 18F), $0.5 \mathrm{~m}$ (19.7 in.) of subsidence was observed in the crack along the western end of the scanned area. Standing water in the crack along the eastern end of the scanned area prevented determination of change for this part of the crack during these periods. Little to no change was observed in the crack in most of the scanned area.

Time-series shaded-relief images show the progression of changes in the crack from January 2009 through December 2016 (fig. 19). During 2010, the crack appeared to be filling; then it opened slightly in a few places during 2011. By 2014, a large sink-like depression had formed near the western end of the scanned area. The crack appeared to be filling in again in 2015 and 2016.

\section{Volumetric Changes}

Using the high-resolution lidar data, changes in the volume of the crack were calculated between the sequential lidar surveys for the west and east ends of the crack (fig. 20A). Volumetric calculations between a fixed reference plane and the land surface were made for each survey (fig. 20B; table 2). The change in crack volume due to subsidence or deposition was estimated as the difference in the calculated surface-toplane volumes between sequential lidar surveys (fig. 20C; table 2). As such, the measured volume change represents the change in void volume defined by the surface of the crack 'visible' from land surface. A positive volume change indicates a relative increase in the volume of the crack. 


\section{A}

\section{January 2009 to September 2010}

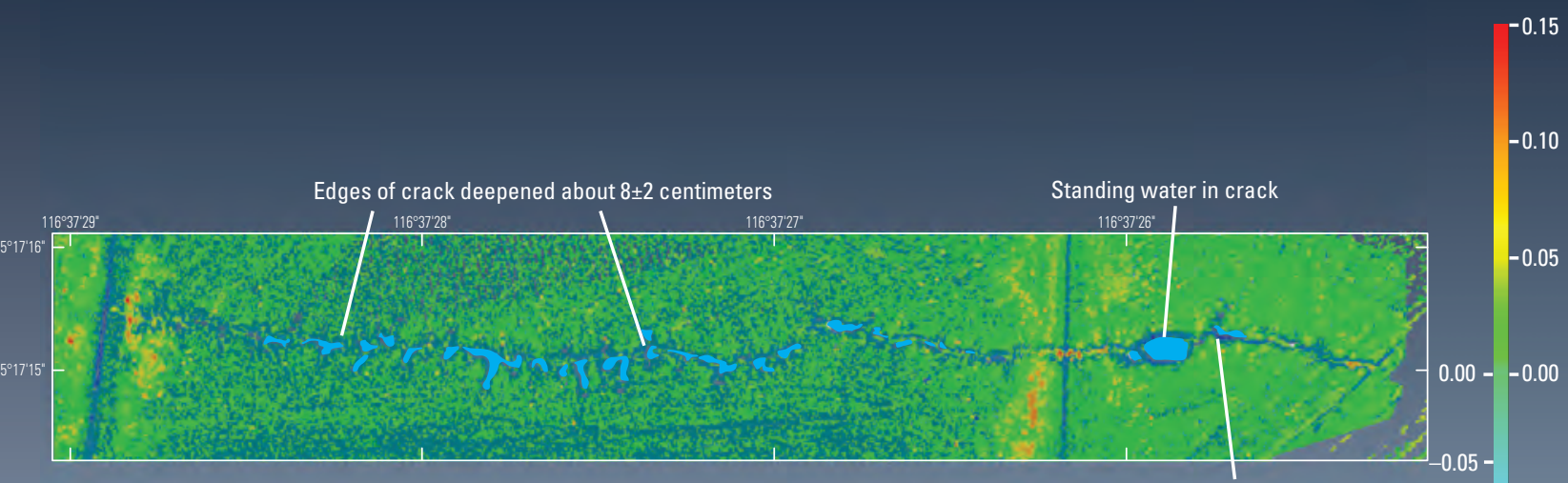

Maximum subsidence was 0.18 meter

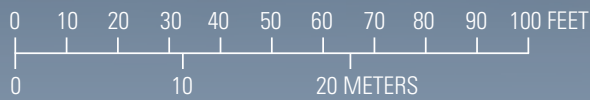

\section{B}

\section{September 2010 to April 2011}
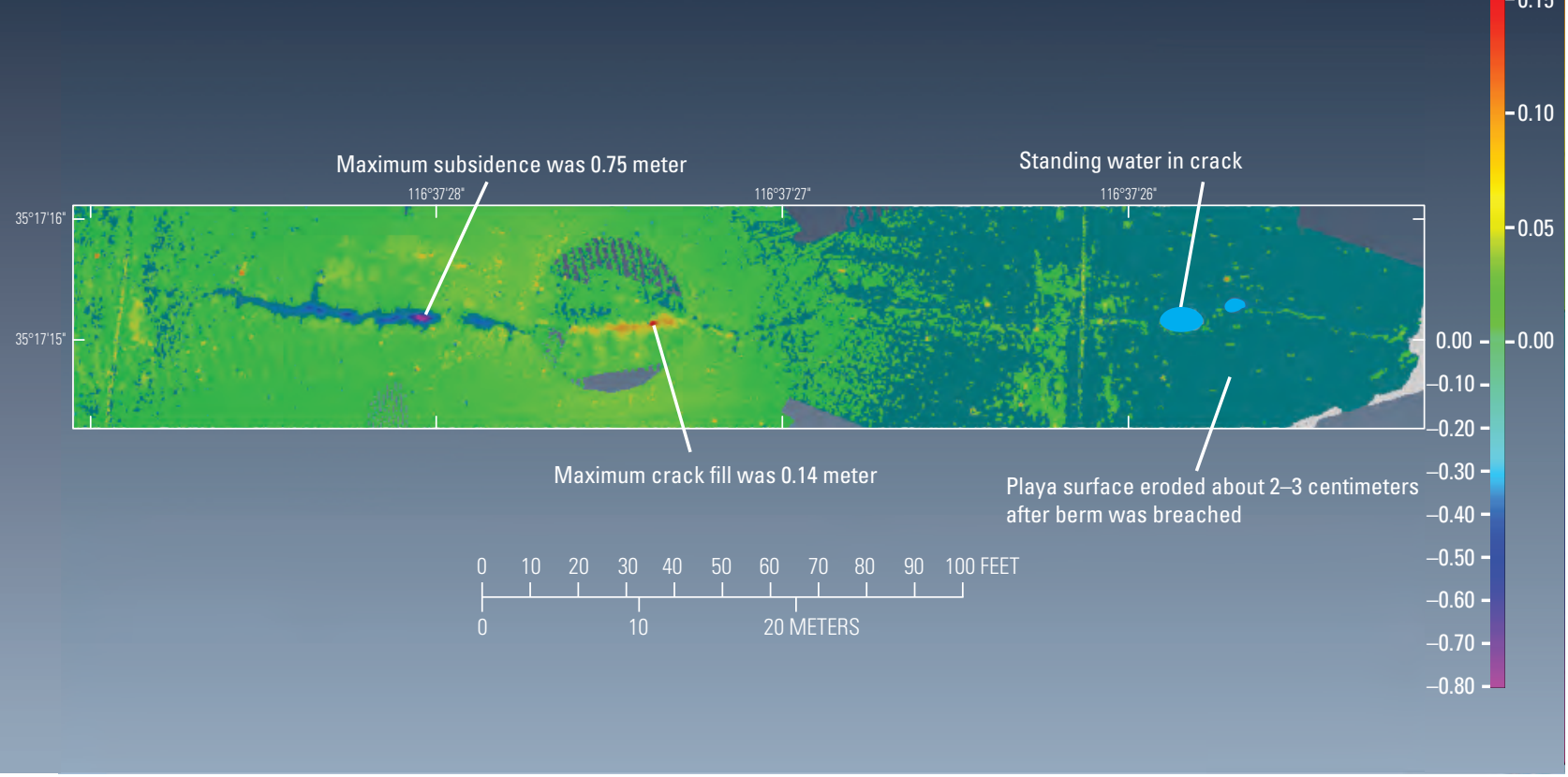

Figure 18. Vertical land-surface change for 2005-06 main-runway crack, Fort Irwin National Training Center, California, for A, January 2009-September 2010; B, September 2010-April 2011; C, April 2011-October 2014; D, October 2014-November 2015; E, November 2015-December 2016; and F, January 2009-December 2016. 
C

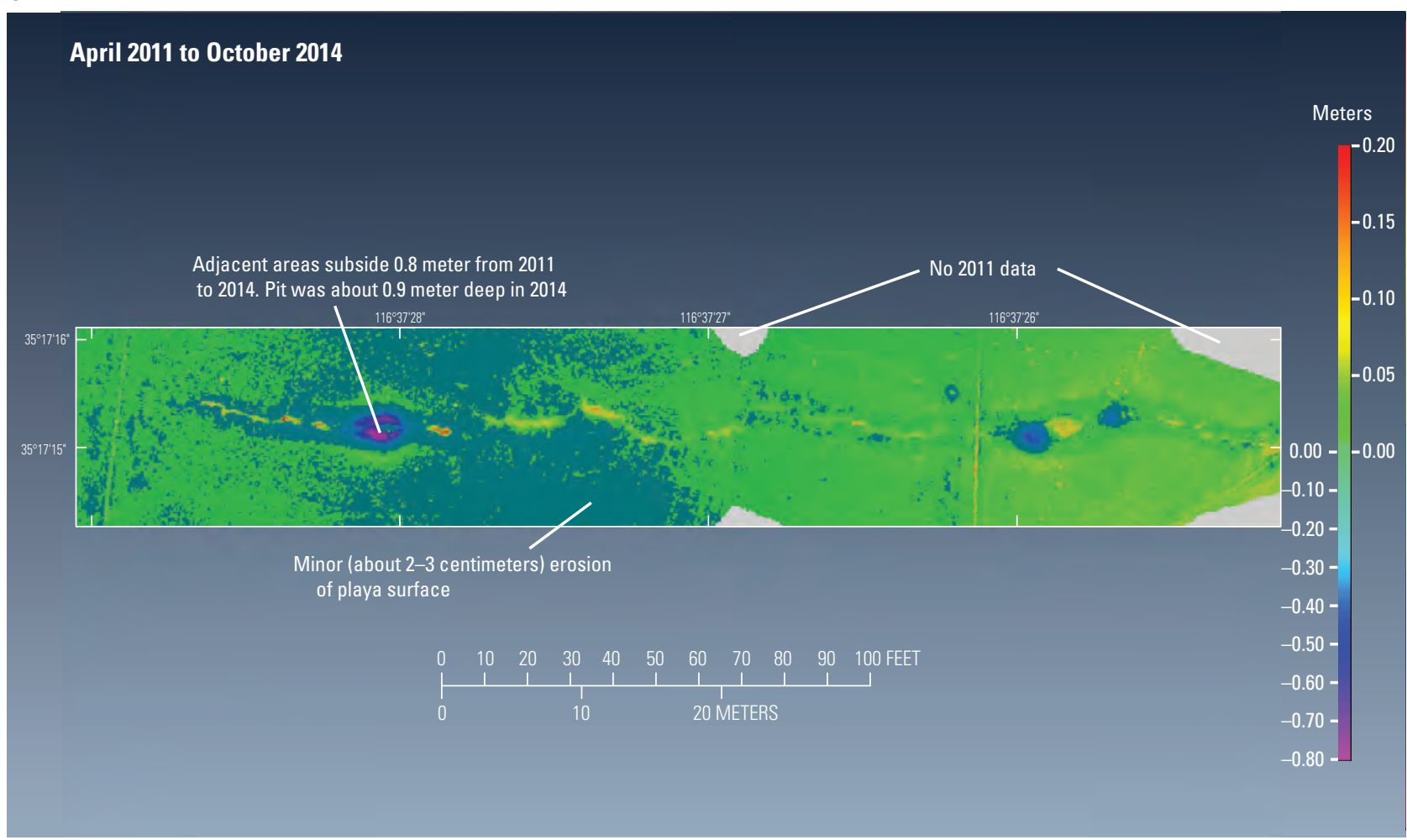

D

October 2014 to November 2015

Meters

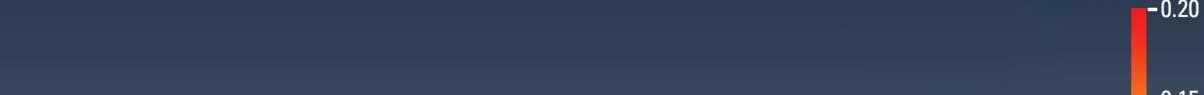

Essentially no vertical change in the playa surface from 2014 to 2015

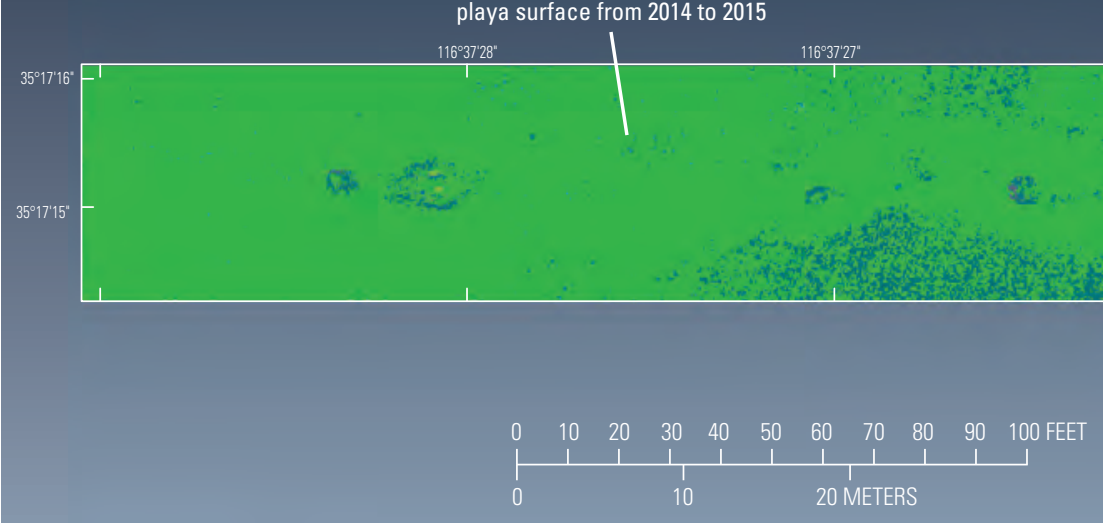

Standing water in crack

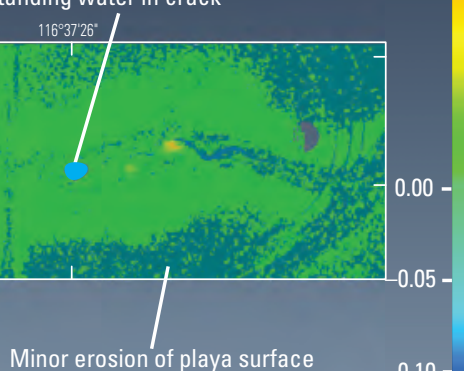

Figure 18. - Continued 


\section{E}

November 2015 to December 2016

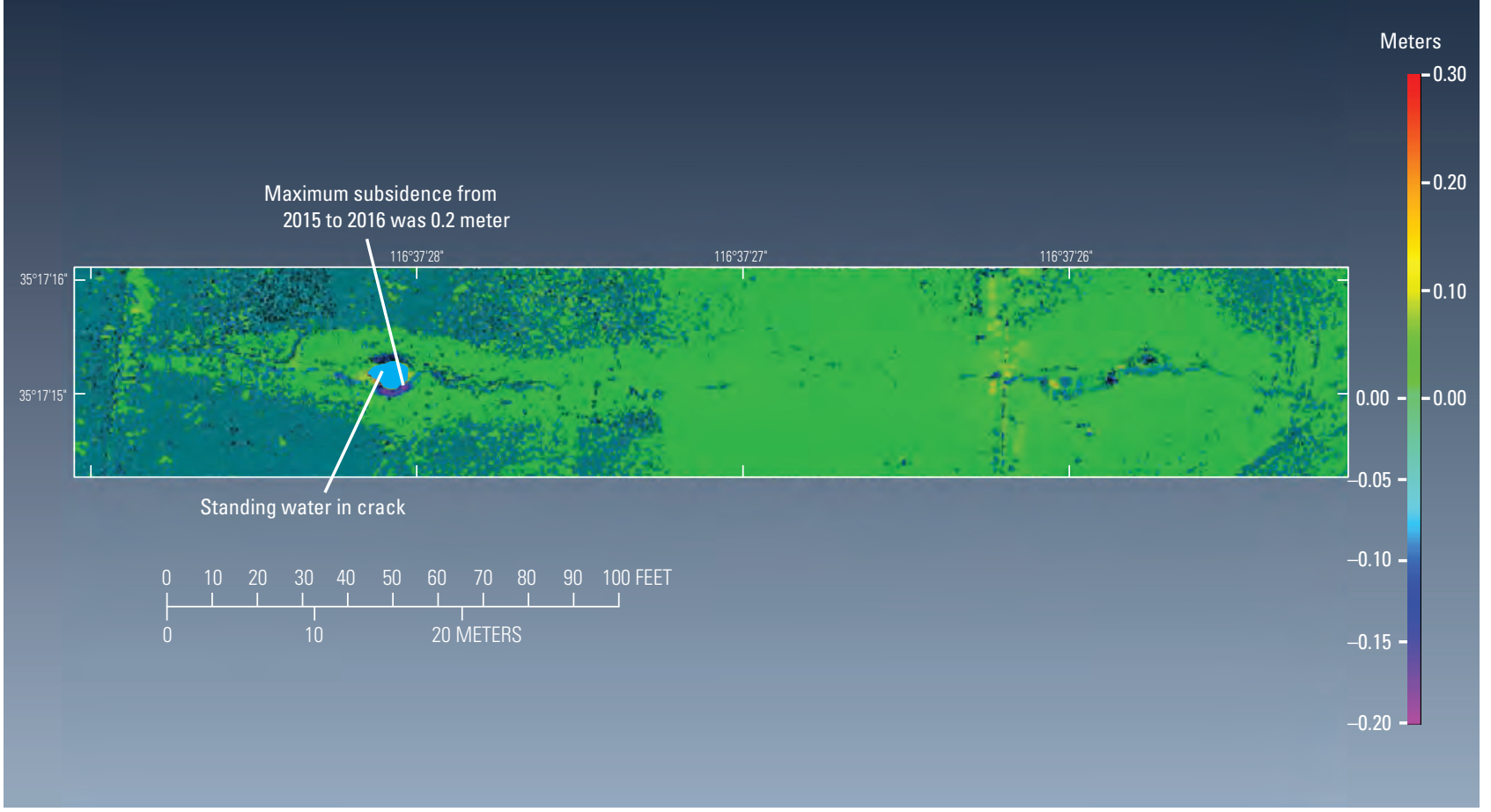

$\boldsymbol{F}$

January 2009 to December 2016

Meters $-0.15$

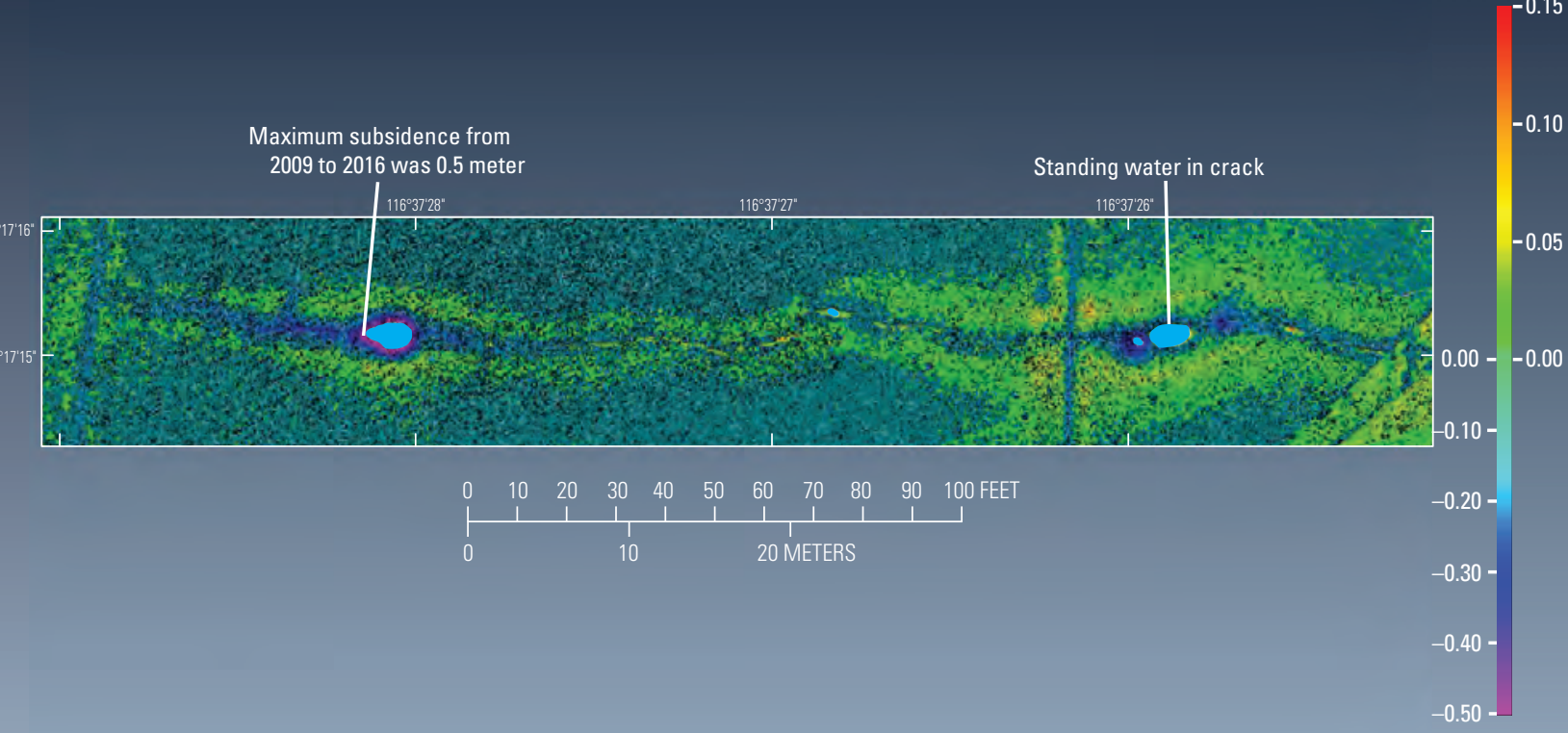

Figure 18. -Continued 


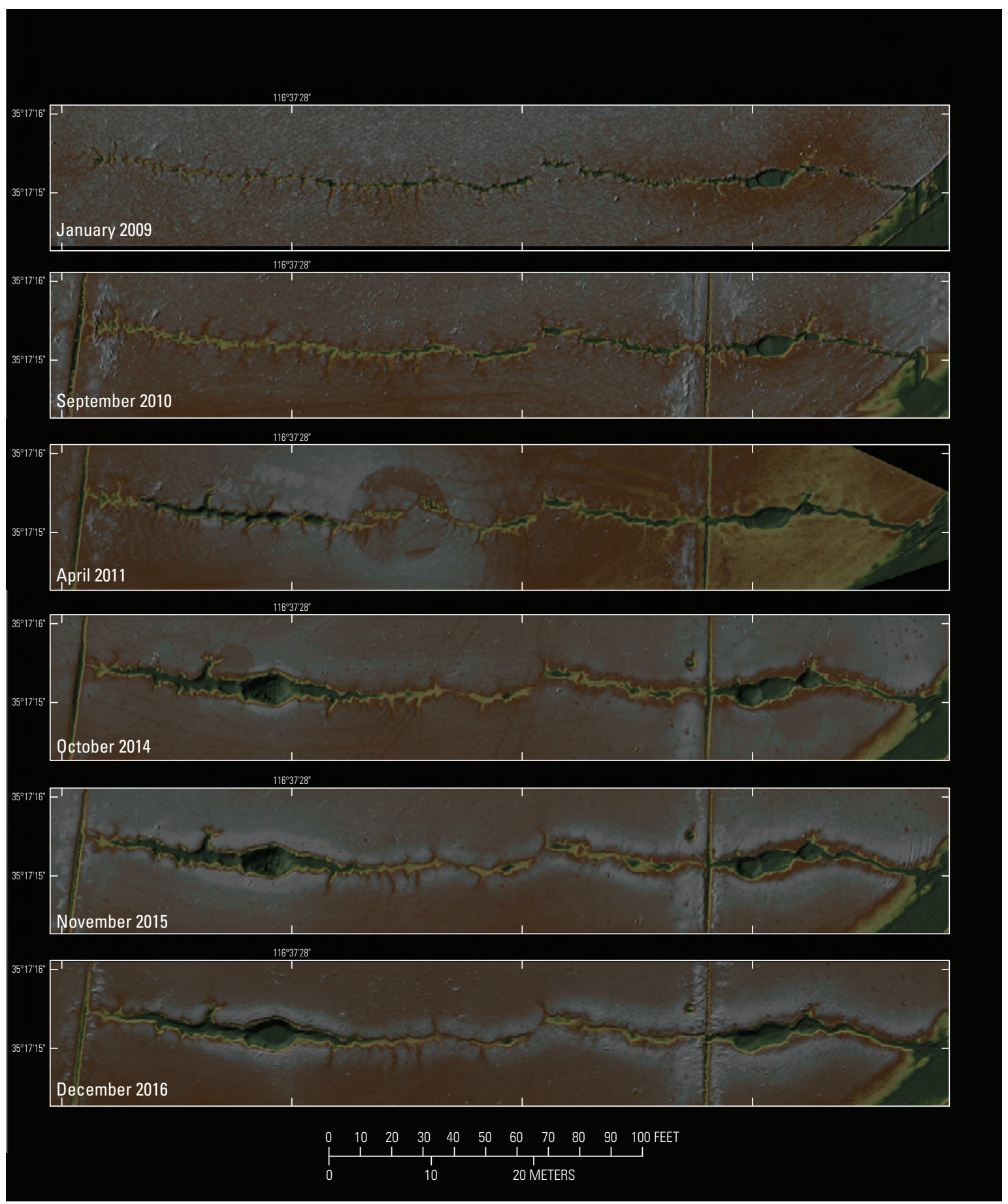

Figure 19. Time-series shaded-relief images for 2005-06 main-runway crack from January 2009 through December 2016, Fort Irwin National Training Center, California. 


\section{A}

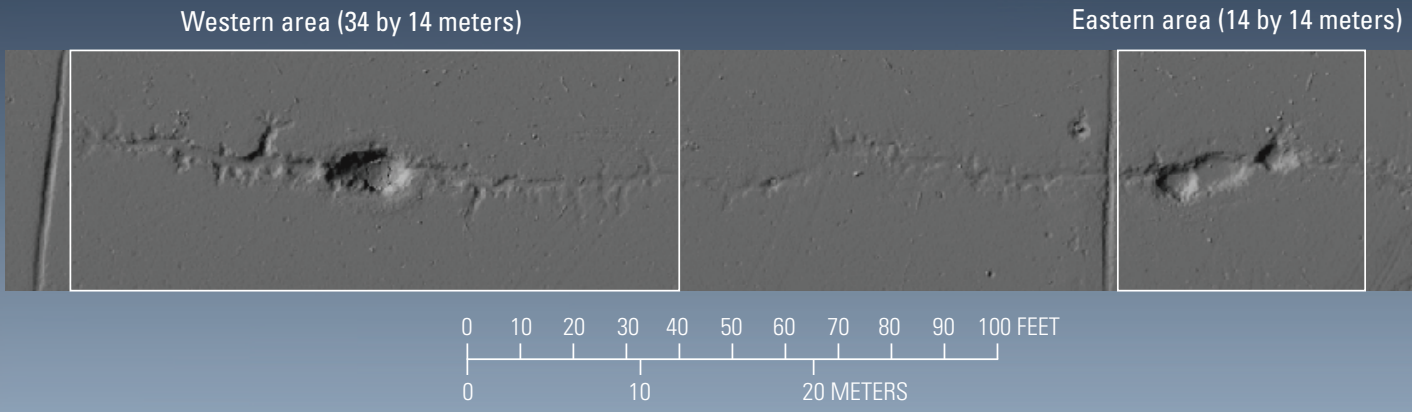

B

Reference plane above and parallel to the playa surface Perimeter of western area projected onto the reference plane

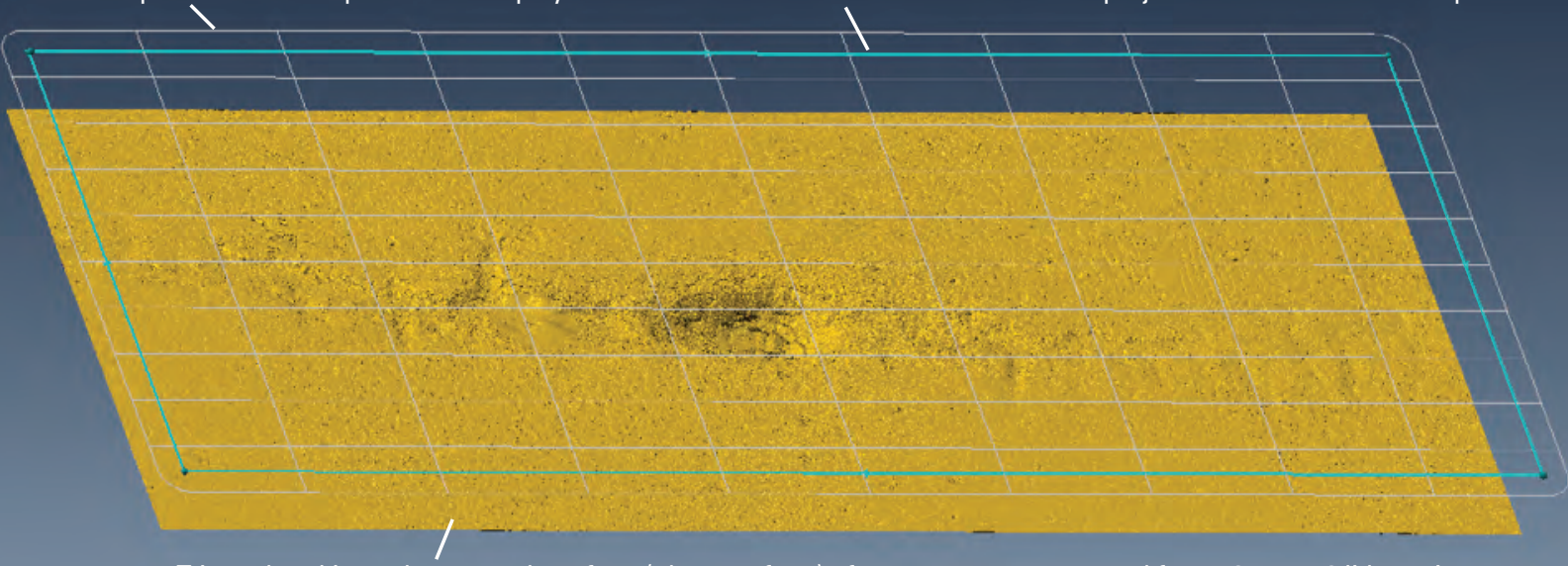

Triangulated irregular network surface (playa surface) of western area generated from 12,741,750 lidar points

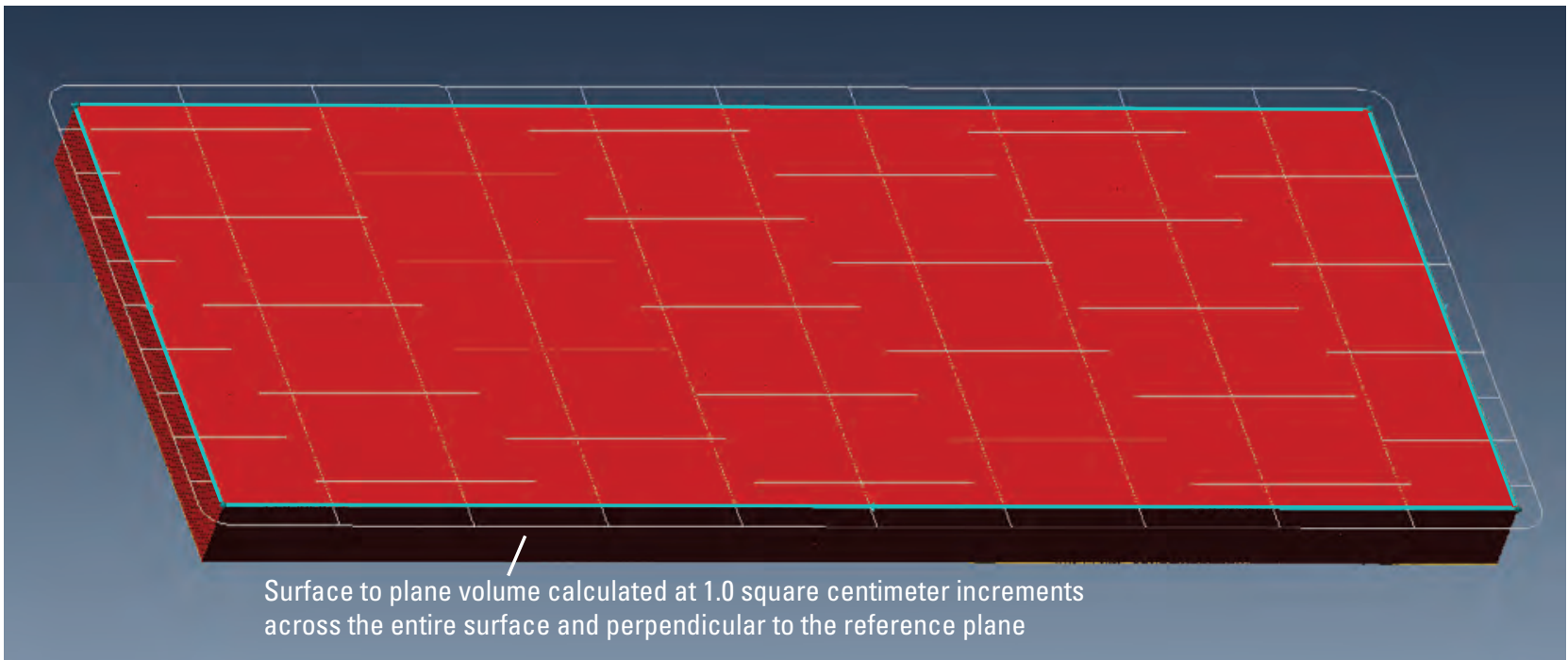

Figure 20. Progression of volumetric calculation along the main-runway crack on Bicycle Lake playa, Fort Irwin National Training Center, California: $A$. west and east areas analyzed for change; $B$. surface and reference planes and volume calculations; and $C$. areas of changes in east and west areas. 
C

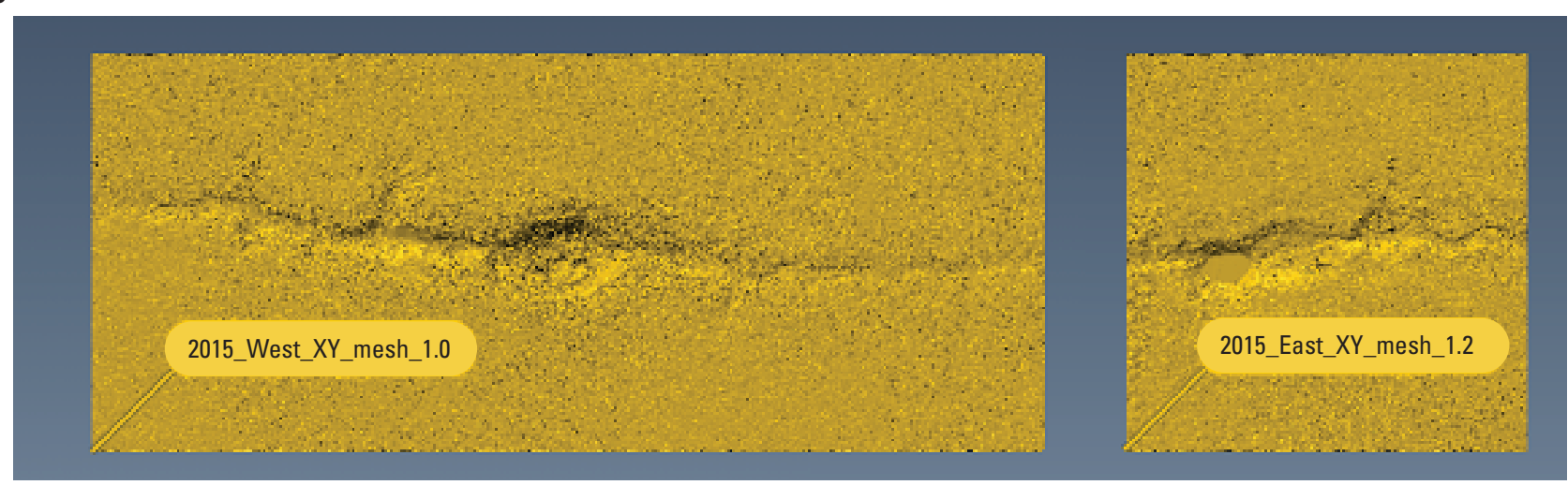

Figure 20. - Continued

Table 2. Summary of the change in volume of the crack calculated between sequential surveys on Bicycle Lake playa, Fort Irwin National Training Center, California.

[ $\mathrm{ft}^{3}$, cubic foot; $\mathrm{m}$, meter; $\mathrm{m}^{2}$, square meter; $\mathrm{m}^{3}$, cubic meter; - , not applicable]

\begin{tabular}{|c|c|c|c|c|c|c|c|c|c|c|}
\hline \multirow[t]{2}{*}{$\begin{array}{l}\text { Surface to } \\
\text { plane }\end{array}$} & \multirow[t]{2}{*}{$\begin{array}{c}\text { Volume } \\
\left(\mathrm{m}^{3}\right)\end{array}$} & \multicolumn{2}{|c|}{ Volume change } & \multicolumn{2}{|c|}{$\begin{array}{c}\text { Cumulative } \\
\text { volume change }\end{array}$} & \multirow[t]{2}{*}{ Year } & \multicolumn{2}{|c|}{$\begin{array}{c}\text { Net volume } \\
\text { change for } \\
\text { West plus East end }\end{array}$} & \multicolumn{2}{|c|}{$\begin{array}{c}\text { Cumulative volume } \\
\text { change for } \\
\text { West plus East end }\end{array}$} \\
\hline & & $\mathbf{m}^{3}$ & $\mathrm{ft}^{3}$ & $\mathbf{m}^{3}$ & $\mathrm{ft}^{3}$ & & $\mathbf{m}^{3}$ & $\mathbf{f t}^{3}$ & $\mathbf{m}^{3}$ & $\mathbf{f t}^{3}$ \\
\hline \multicolumn{11}{|c|}{ West area $\left(14 \mathrm{~m} \times 34 \mathrm{~m}=476 \mathrm{~m}^{2}\right)$} \\
\hline 2009 West end & 953.9 & - & - & - & - & - & - & - & - & - \\
\hline 2010 West end & 955.5 & 1.6 & 56 & - & - & 2010 & 2.0 & 69 & - & - \\
\hline 2014 West end & 960.2 & -2.0 & -69 & 6.2 & 220 & 2014 & -0.8 & -30 & 7.6 & 268 \\
\hline 2015 West end & 959.8 & -0.3 & -11 & 5.9 & 209 & 2015 & 0.9 & 33 & 8.5 & 301 \\
\hline 2016 West end & 953.8 & -6.1 & -215 & -0.2 & -6 & 2016 & -7.0 & -247 & 1.5 & 54 \\
\hline \multicolumn{11}{|c|}{ East area $\left(14 \mathrm{~m} \times 14 \mathrm{~m}=196 \mathrm{~m}^{2}\right)$} \\
\hline 2014 East end & 405.2 & 1.1 & 39 & 1.4 & 48 & - & - & - & - & - \\
\hline 2015 East end & 406.5 & 1.3 & 44 & 2.6 & 92 & - & - & - & - & - \\
\hline 2016 East end & 405.6 & -0.9 & -32 & 1.7 & 60 & - & - & - & - & - \\
\hline
\end{tabular}

From 2009 to 2010, the change in volume was about $1.6 \mathrm{~m}^{3}\left(56 \mathrm{ft}^{3}\right)$ along the west end of the crack and about $0.4 \mathrm{~m}^{3}\left(13 \mathrm{ft}^{3}\right)$ along the east end, for a total increase of $2 \mathrm{~m}^{3}\left(69 \mathrm{ft}^{3}\right)$. Between 2010 and 2011, the volume enlarged $6.6 \mathrm{~m}^{3}\left(233 \mathrm{ft}^{3}\right)$ on the west end and infilled $0.1 \mathrm{~m}^{3}\left(4 \mathrm{ft}^{3}\right)$ on the east end, for a net volume increase of $6.5 \mathrm{~m}^{3}\left(228 \mathrm{ft}^{3}\right)$. The cumulative volume increase (equivalent subsidence) from 2009 to 2011 was $8.4 \mathrm{~m}^{3}\left(297 \mathrm{ft}^{3}\right)$.

Between 2011 and 2014, a prominent pit formed along the western end of the crack. Despite the formation of this $0.8 \mathrm{~m}$ (31 in.) deep pit, about $2 \mathrm{~m}^{3}\left(69 \mathrm{ft}^{3}\right)$ of sediment filled in the western part of the crack. During the same period, two additional pits formed along the eastern end of the crack, which presumably caused $1.1 \mathrm{~m}^{3}\left(39 \mathrm{ft}^{3}\right)$ of sediment to be lost to the subsurface. The net volume change from 2011 to 2014 was $-0.8 \mathrm{~m}^{3}\left(-30 \mathrm{ft}^{3}\right)$, representing infilling (deposition), which brought the cumulative 2009-14 volume increase to $7.6 \mathrm{~m}^{3}\left(268 \mathrm{ft}^{3}\right)$.

By 2015 , another $0.3 \mathrm{~m}^{3}\left(11 \mathrm{ft}^{3}\right)$ of sediment had been deposited in the western part of the crack, whereas the eastern end had surface subsidence equivalent to $1.3 \mathrm{~m}^{3}\left(44 \mathrm{ft}^{3}\right)$. From 2009 to 2015 , the cumulative volumetric increase was $8.5 \mathrm{~m}^{3}$ $\left(301 \mathrm{ft}^{3}\right)$. This volume change is a minimum estimate because the crack volume below the water surface in the pits was not included in the calculations. 
By $2016,6.1 \mathrm{~m}^{3}\left(215 \mathrm{ft}^{3}\right)$ of sediment had been deposited into the western part of the crack, whereas the eastern end had infilled about $0.9 \mathrm{~m}^{3}\left(32 \mathrm{ft}^{3}\right)$. From 2009 to 2016, the cumulative volumetric increase was $1.5 \mathrm{~m}^{3}\left(54 \mathrm{ft}^{3}\right)$. This volume change is a minimum estimate because the crack volume below the water surface in the pits was not included.

\section{Interferometric Synthetic Aperture Radar}

\section{Land Subsidence}

Of the 103 interferograms of Bicycle Basin analyzed for this study, 90 show land-surface elevation losses, or subsidence, in the area immediately north of Bicycle Playa in the northwestern part of Bicycle Basin (table 1). Of the 13 interferograms that indicate no deformation, 8 of them span about 2 months or less. The remaining five interferograms were for periods of expected water-level recovery (for example, interferogram 80 in table 1). It is likely there was subsidence during at least some of these interferogram periods, particularly those of expected groundwater-level declines (for example, interferogram 73 in table 1), but the magnitudes were too small over the short period to be detectable using InSAR. The area affected by subsidence was about $6.7 \mathrm{~km}^{2}\left(2.6 \mathrm{mi}^{2}\right)$ and coincided with an area containing substantial clay deposits, as documented in drillers' logs for wells 14N/3E-23B1, -14P1, -23G1, and -14P2 (figs. 4B, 21). Subsidence was not detected elsewhere in the basin, where logs for wells 14N/3E-24Q1, -24H1, -18N1, -13M2, -13M1, $-13 \mathrm{~K} 1$, and $-14 \mathrm{H} 1$ indicate that clay deposits are much less prevalent (figs. $4 B, 21 A-E$ ).

Subsidence rates in Bicycle Basin calculated from interferograms spanning 1992-97 range from 0.7 to $2.7 \mathrm{~mm} / \mathrm{mo}(0.03-0.11 \mathrm{in} . / \mathrm{mo})$ and average $1.6 \mathrm{~mm} / \mathrm{mo}$ (0.06 in./mo; table 1). Subsidence rates calculated from interferograms spanning 1998-2007 (excluding the 35-day interferogram that showed no subsidence) range from 1.0 to $4.4 \mathrm{~mm} / \mathrm{mo}(0.04-0.18 \mathrm{in} . / \mathrm{mo})$ and average $2.6 \mathrm{~mm} / \mathrm{mo}(0.11 \mathrm{in} . / \mathrm{mo}$; table 1$)$. Subsidence rates calculated from interferograms spanning 2008-15 (excluding the interferograms that showed no subsidence) range from 0.4 to $3.0 \mathrm{~mm} / \mathrm{mo}(0.01-0.12 \mathrm{in} . / \mathrm{mo})$ and average $1.5 \mathrm{~mm} / \mathrm{mo}$ (0.06 in. $/ \mathrm{mo}$; table 1). These data indicate that subsidence rates during $1992-97$ and $2008-15$ were nearly half the rates during 1998-2007.

Subsidence rates calculated from interferograms are often affected by the seasonal hydrologic conditions in which each SAR image was acquired. This temporal relation to subsidence rate is likely a result of seasonal water-level fluctuations in the pumping zone. On an annual or multi-annual time scale in basins where pumpage is less than or equal to recharge, it can be expected that water levels during a specific month are similar from year to year, and as a result, annual subsidence is not expected. In an aquifer system comprising a large fraction of fine-grained (clays and silts) deposits where more water is removed than is replenished, as in Bicycle Basin, annual water-level declines and associated subsidence can be expected. For interferograms produced from two SAR images acquired during the same season spanning one or more years in Bicycle Basin (for example, fig. 21A), subsidence rates were expected to be close to the average computed from all available interferograms. For the three interferograms that span annual or multi-annual periods during 1992-97, the rates of two of them were close to the average rate (for example, interferogram 4 in table 1), and one was lower than the average rate for this period $(1.6 \mathrm{~mm} / \mathrm{mo}$ or $0.06 \mathrm{in} . / \mathrm{mo})$. For the 14 interferograms that span annual or multi-annual periods during 1998-2007 (excluding one that indicated no subsidence), the rates of nine were close to the average rate (for example, interferogram 37 in table 1), three were lower than the average rate, and two were higher than the average rate for this period $(2.6 \mathrm{~mm} / \mathrm{mo}$ or $0.11 \mathrm{in} . / \mathrm{mo})$. For the 19 interferograms that span annual or multi-annual periods during 2008-15, the rates of nine were close to the average (for example, interferogram 71 in table 1), six were lower than the average rate, and four were higher than the average rate for this period $(1.5 \mathrm{~mm} / \mathrm{mo}$ or $0.06 \mathrm{in} . / \mathrm{mo})$.

Where water-levels fluctuate seasonally, the timing of data acquisition can greatly affect the computed subsidence rates. If the first image is acquired when water levels are near seasonal highs (winter or spring) and the second image is acquired when water levels are near seasonal lows (summer or fall), higher than average subsidence rates would be expected. Conversely, if the first image is acquired when water levels are near seasonal lows and the second image is acquired when water levels are near seasonal highs, lower than average subsidence rates, or even uplift, would be expected.

In Bicycle Basin, pumping generally is greater during the summer and fall, resulting in seasonally lowered water levels of as much as $12 \mathrm{~m}$ (40 ft; for example, 14N/3E-14P2 in fig. 15A) compared with those during the winter and spring, when pumping generally is less. Of the six interferograms for 1992-97 for which the second SAR acquisition was expected to be associated with lower water levels than for the first (for example, interferogram 9 in table 1 and fig. $21 B$ ), all but one showed average or greater subsidence rates (for example, interferogram 11 in table 1). Of the 26 interferograms for 1998-2007 associated with seasonally lower water levels, 21 indicated average or greater rates of subsidence (for example, interferogram 39 in table 1). Of the 30 interferograms for $2008-15$ associated with seasonally lower water levels, 23 indicated average or greater subsidence rates (interferogram 72 in table 1). When the first image represents water levels near seasonal lows and the second image represents water levels near seasonal highs, however, average or lower subsidence rates, or even uplift, are expected. 
$\boldsymbol{A}$

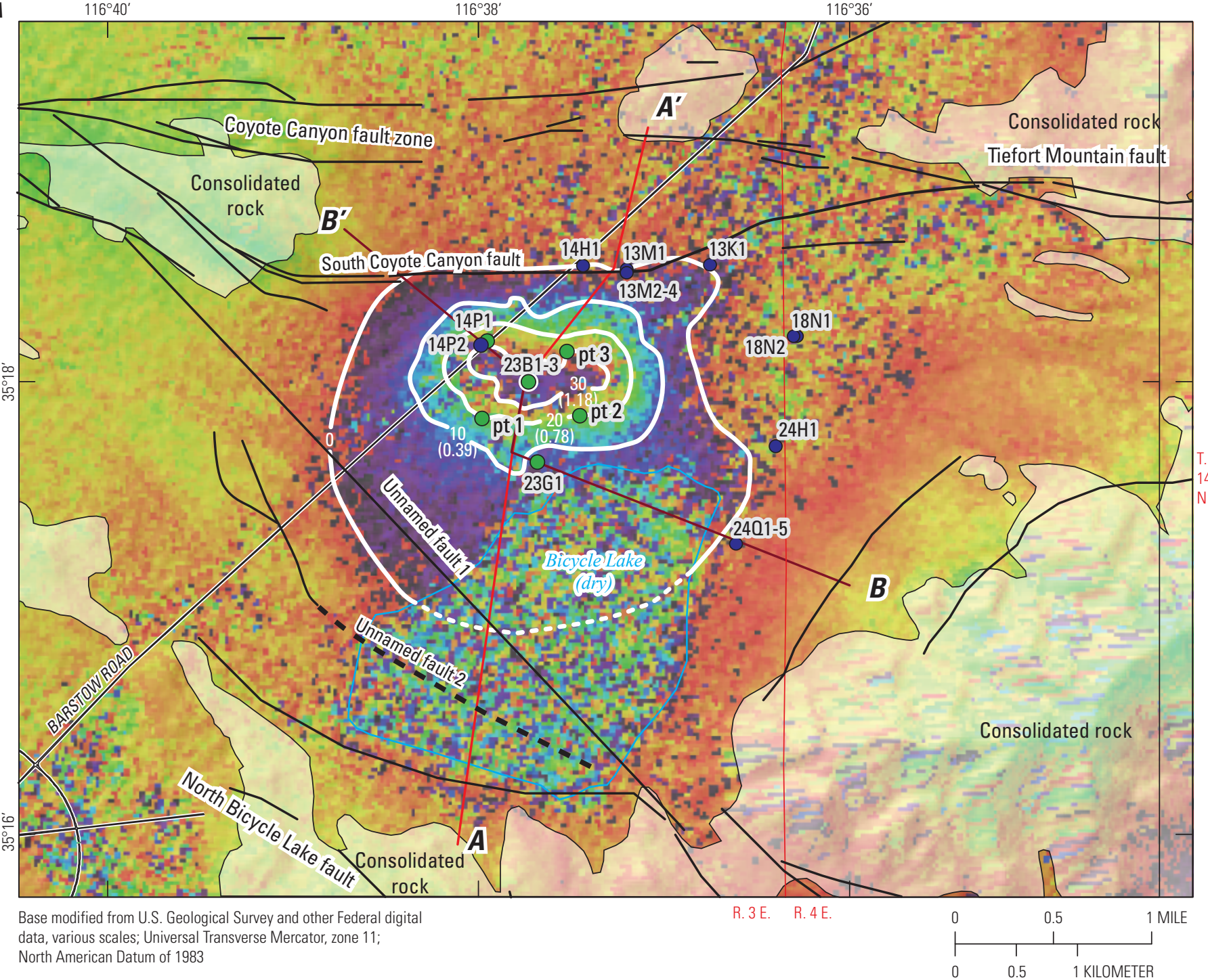

EXPLANATION
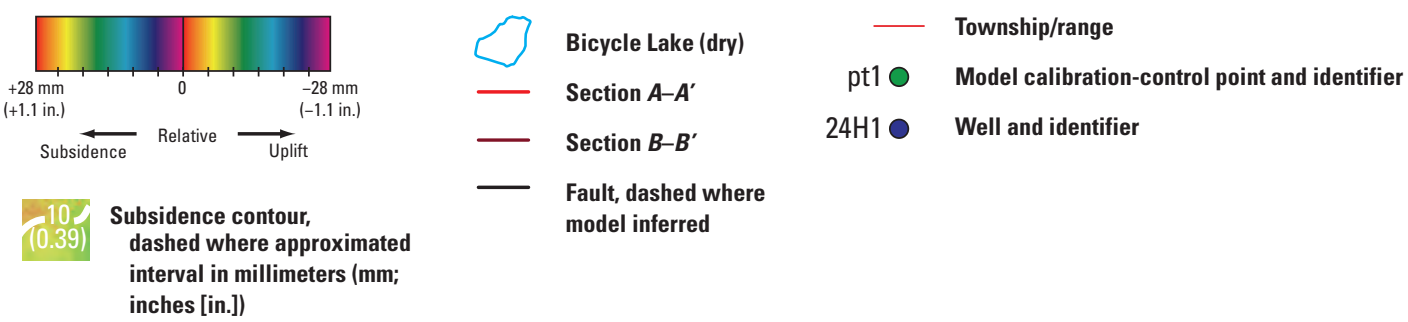

ection $B-B^{\prime}$

24H1 Well and identifier

Fault, dashed where model inferred

Figure 21. Subsidence for Bicycle Basin, Fort Irwin National Training Center, California: $A$, November 30, 2003-January 23, 2005 (\#25 in table 1); B, March 27, 2000-September 18, 2000 (\#18 in table 1); C, July 17, 2005-April 23, 2006 (\#48 in table 1); D, June 20, 2013-June 23, 2014 (\#77 in table 1); and $E$, October 29, 2014-0ctober 24, 2015 (\#101 in table 1). 


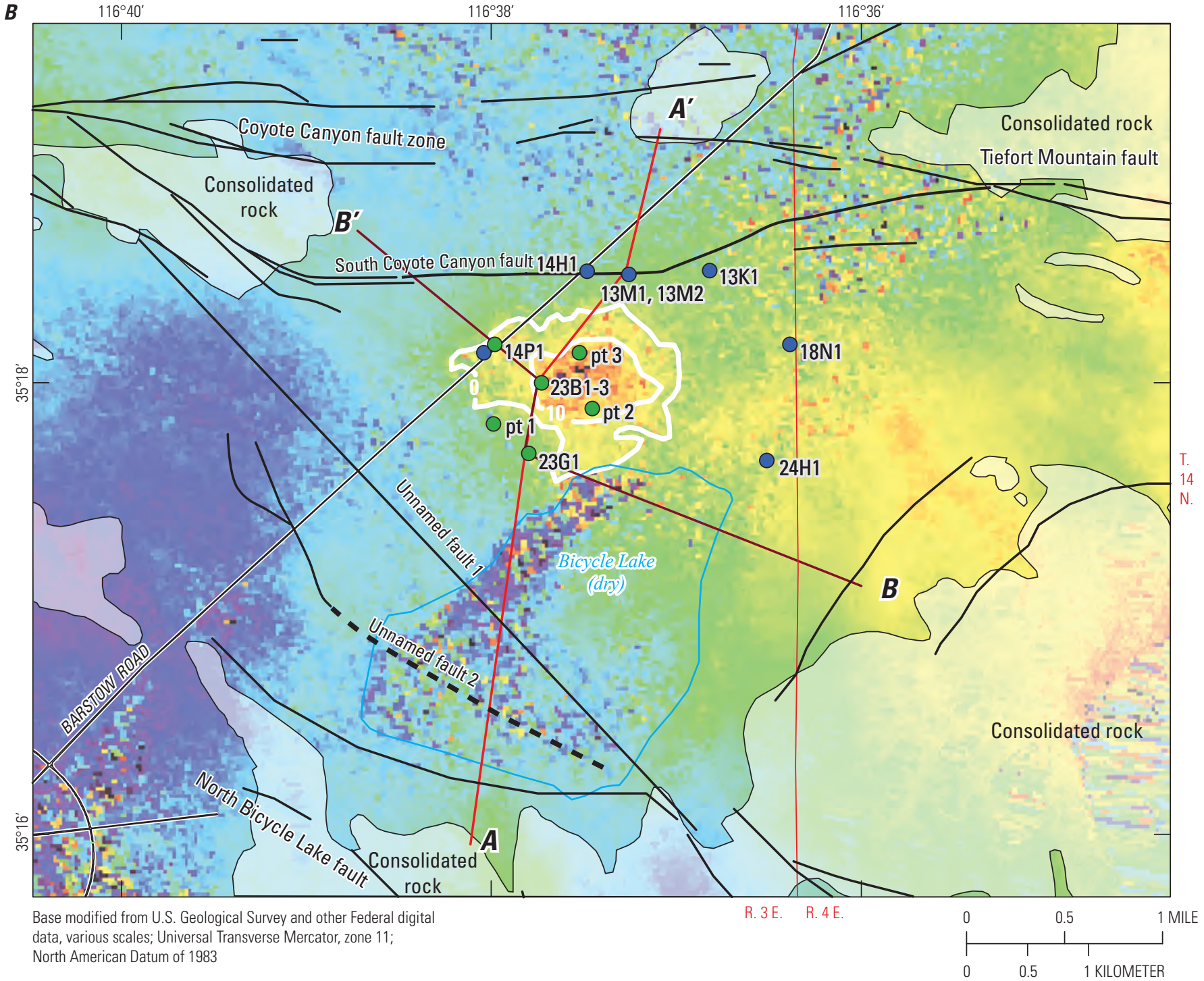

data, various scales; Universal Transverse Mercator, zone 11;

North American Datum of 1983

\section{EXPLANATION}

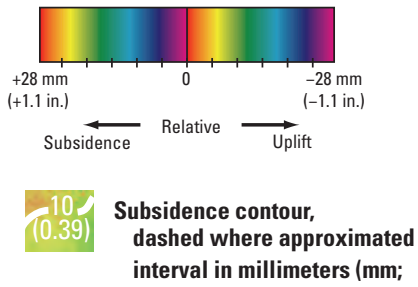

$\int$ Bicycle Lake (dry)

Section $\boldsymbol{A}-\boldsymbol{A}^{\prime}$

- Fault

\section{pt1 Model calibration-control point (pt) and identifie \\ $24 \mathrm{H} 1 \bigcirc \quad$ Well and identifier}

Figure 21. - Continued 


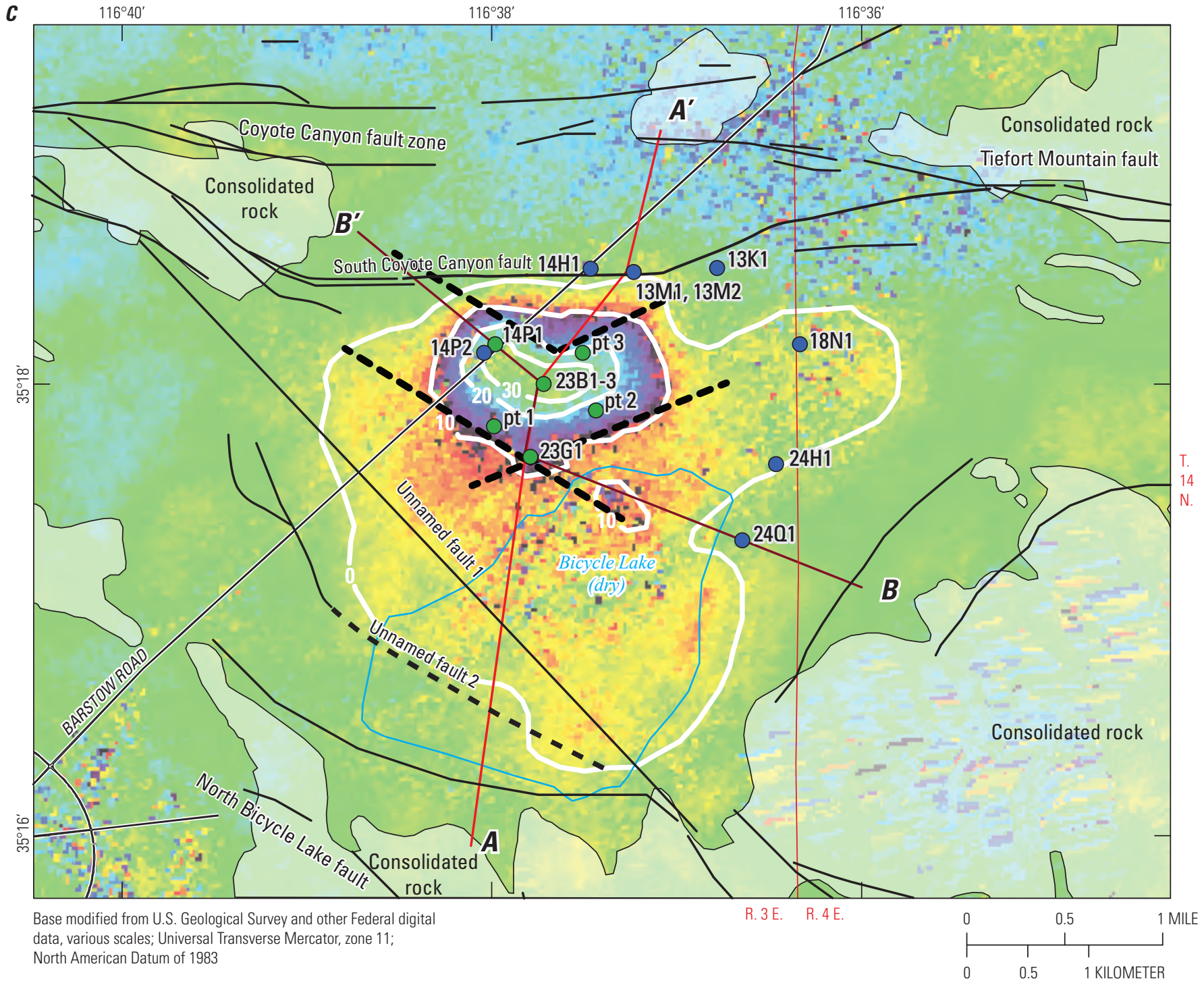

\section{EXPLANATION}

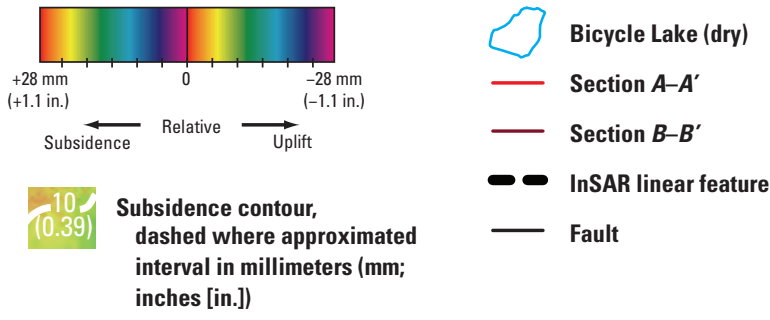

O Model calibration-control point (pt) and identifier

- Well and identifier

Figure 21. - Continued 
D

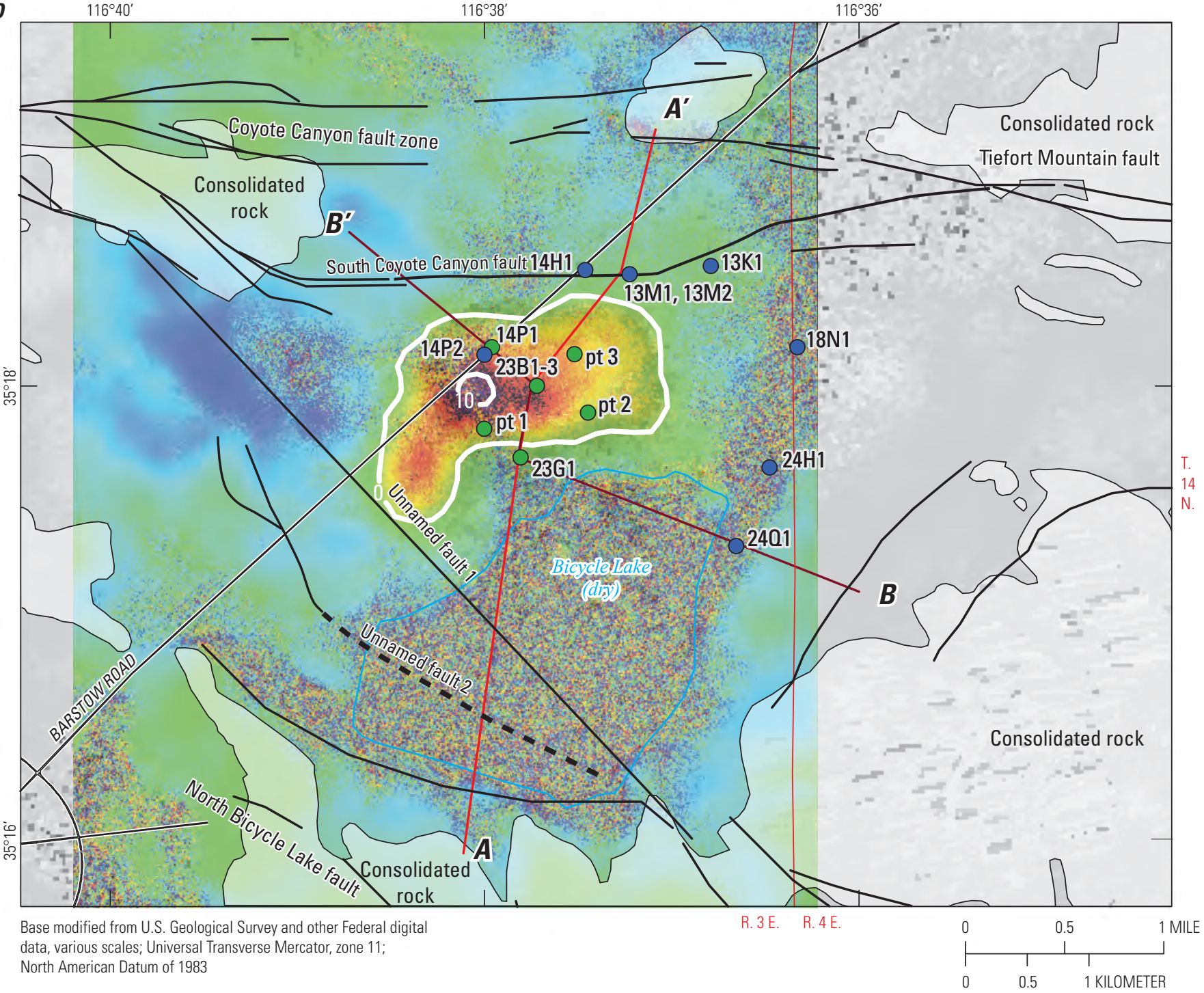

EXPLANATION
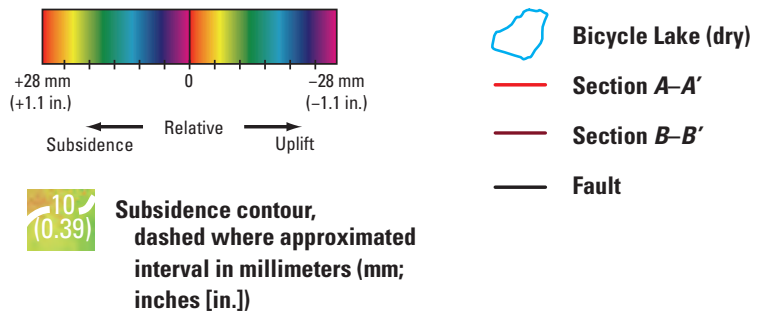

Township/range

Model calibration-control point (pt) and identifier

Well and identifier

Figure 21. - Continued 

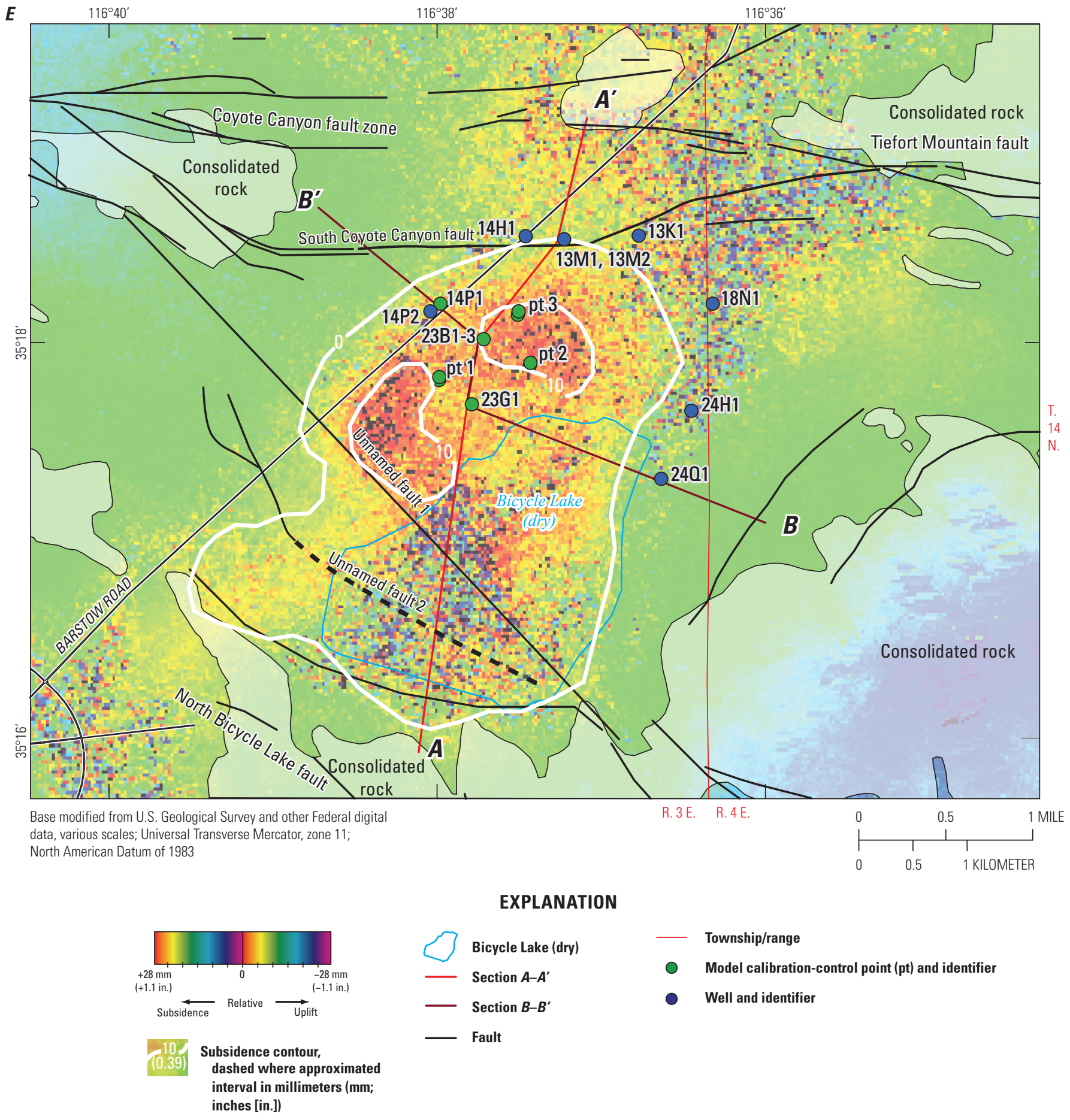

\section{EXPLANATION}

\begin{tabular}{lll} 
Bicycle Lake (dry) & & Township/range \\
Section $A-A^{\prime}$ & $\bigcirc$ & Model calibration-control point (pt) and identifier \\
$\begin{array}{l}\text { Section } B-B^{\prime} \\
\text { Fault }\end{array}$ & $\bigcirc$ & Well and identifier \\
\hline
\end{tabular}

Figure 21. -Continued 
Of the two interferograms for 1992-97 for which the first SAR acquisition was during summer or fall and the second during winter or spring, both showed average subsidence rates (for example, interferogram 5 in table 1). For the 18 interferograms for 1998-2007 during which water-level recovery was expected, 10 indicated average or lower rates of subsidence (for example, interferograms 19 in table 1). For the nine interferograms for 2008-15 during which water levels were expected to have recovered, seven indicated average or lower subsidence rates (for example, interferogram 76 in table 1). These results indicate that subsidence rates generally were higher during the summer and fall, when water levels were expected to decline, and were lower during the winter and spring, when water levels were expected to recover.

Although water levels were generally measured infrequently during the 1990s and early 2000s, pumpage records indicate substantial variation in pumping volume between summer and winter (fig. 22), indicating that water levels were seasonally affected. Substantial seasonal fluctuations in water levels could have contributed to the more variable subsidence rates measured by InSAR during the 1990s and 2007-15 than measured during 2003-06, when seasonal variations were less and annual volumes of pumping were larger (fig. 22). Additionally, pumpage data indicate substantially less pumping during 2013-14 throughout the basin, and less subsidence was measured during the same period.

Subsidence rates also could be affected by variable pumping on relatively short time scales. For example, water levels measured continuously (every half-hour) in well 14N/2E-23B2 indicated declines of as much as

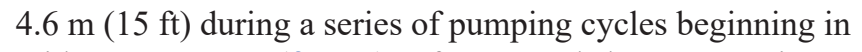
mid-January 2008 (fig. 22). After several shorter pumping and non-pumping cycles through the rest of March and April, water levels recovered to mid-March levels.

Because of the annual, seasonal, and short-term waterlevel fluctuations, and because water levels generally were not measured concurrently with InSAR data, water levels at the time of InSAR data acquisitions are unknown. Based on the water-level data that has been collected intermittently in the 1990s and early 2000s and the more frequent water-level data collected between 2008 and 2015, however, water levels in the production zone generally were tens of feet higher in the winter than in the summer. Thirty-six interferograms analyzed for this study between 1992 and 2015 span periods when water levels likely were recovering, and most indicated subsidence (for example, interferograms 12, 32, 90 in table 1). Subsidence during water-level recovery likely is caused by residual compaction in thick clay layers. The likelihood of residual compaction is supported by the lithologies of wells $14 \mathrm{~N} / 3 \mathrm{E}-14 \mathrm{P} 2$ and $-23 \mathrm{~B} 1$, which indicate clay layers ranging from 3 to $24 \mathrm{~m} \mathrm{(10-80} \mathrm{ft)} \mathrm{thick} \mathrm{and} \mathrm{totaling} 87$ and $70 \mathrm{~m}(285$ and $230 \mathrm{ft}$ ), respectively (figs. 4A, B). Additional evidence for residual compaction is the fact that the numerous seasonal (summer-fall-winter) recovery period interferograms showed no uplift.

Time-series graphs of subsidence for five selected locations were generated for model calibration using 20 interferograms (Densmore and others, 2018). Data from these interferograms showed more than $400 \mathrm{~mm}$ (16 in.) of subsidence during 1993-2014. The time series was constructed such that the end date for one interferogram was the beginning date (or nearly so) for the next interferogram. Where data gaps or overlaps existed, subsidence rates were used to estimate magnitudes for the time interval of the gap or overlap, and the time series were adjusted accordingly. Based on the data, subsidence continued uninterrupted during the gaps. Time series constructed for the five locations indicated that subsidence ranged from about $192 \mathrm{~mm}$ (7.5 in.) at point 1 to about $420 \mathrm{~mm}$ (16.5 in.) at well 14N/3E-23B1 (fig. 23).

\section{Inferred Structure}

Measurements of land-surface subsidence can be used to infer the presence of buried faults not readily evident in surface expression (Galloway and others, 1999; Galloway and Hoffmann, 2007). In alluvial basins, faults are typically barriers to groundwater flow; therefore, water-level changes and related land-surface deformation are greatest on the side of the fault that is pumped (Galloway and others, 1999). Some of the interferograms, particularly those that span periods from 2003 to 2006 that coincided with periods of sustained groundwater withdrawal, showed distinct linear features at the outer edges of the subsidence area, indicating the presence of faults or abrupt changes in lithology affecting the extent of subsidence (fig. 21C). During periods when pumping in winter months was reduced relative to the bracketing summer months (1993-97 and 2007-12 in fig. 22), linear features in the InSAR imagery were less apparent or non-existent. This indicates that changes in the timing and total annual volume of groundwater withdrawal can affect the conditions manifesting as linear features in InSAR imagery. Model-inferred fault locations (figs. 21C), however, correlated with subsidence patterns noted in most interferograms used in this study and appeared to, in part, control the lateral extent of subsidence (fig. 21E). Although the bounding faults had not been previously mapped in the basin (as is common given the difficulty of detecting faults in unconsolidated material using traditional geologic mapping techniques), the agreement between the modelinferred fault locations and subsidence patterns noted in the InSAR imagery indicated the faults in the surrounding hills extend into the basin and control the lateral extent of the subsidence. 

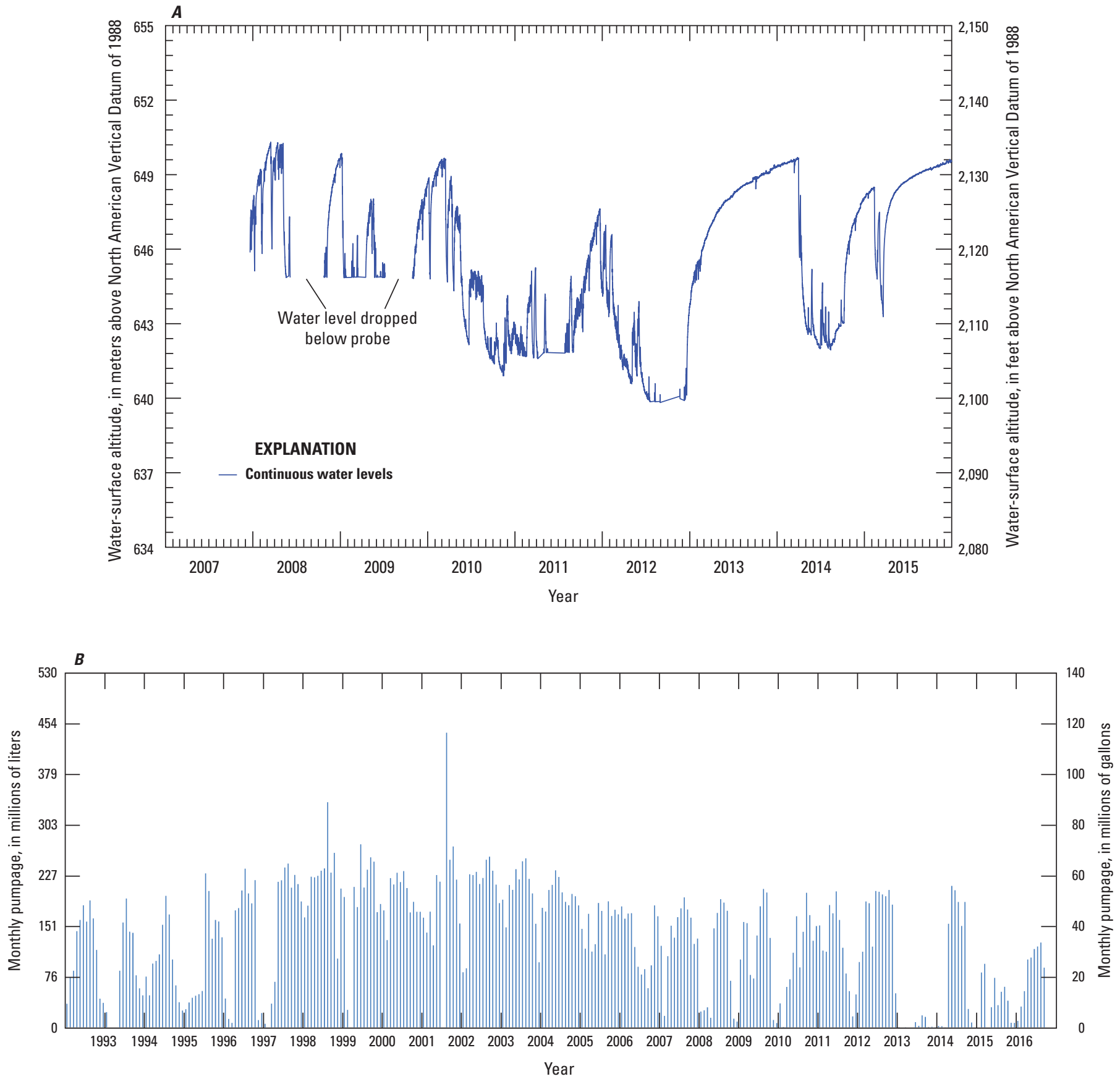

Figure 22. Relationship between water levels near the area of maximum subsidence and monthly pumpage for wells in Bicycle Basin, Fort Irwin National Training Center, California: $A$, water levels for well 14N/3E-23B2 (screen interval 134-140 meters, or 440-460 feet, below land surface), near area of maximum subsidence; and $B$, combined monthly pumpage (Chris Woodruff, Fort Irwin, written commun., 2017) for wells 14N/3E-13K1, -13M1, -14H1, -14P1, and 14N/4E-18N1. 


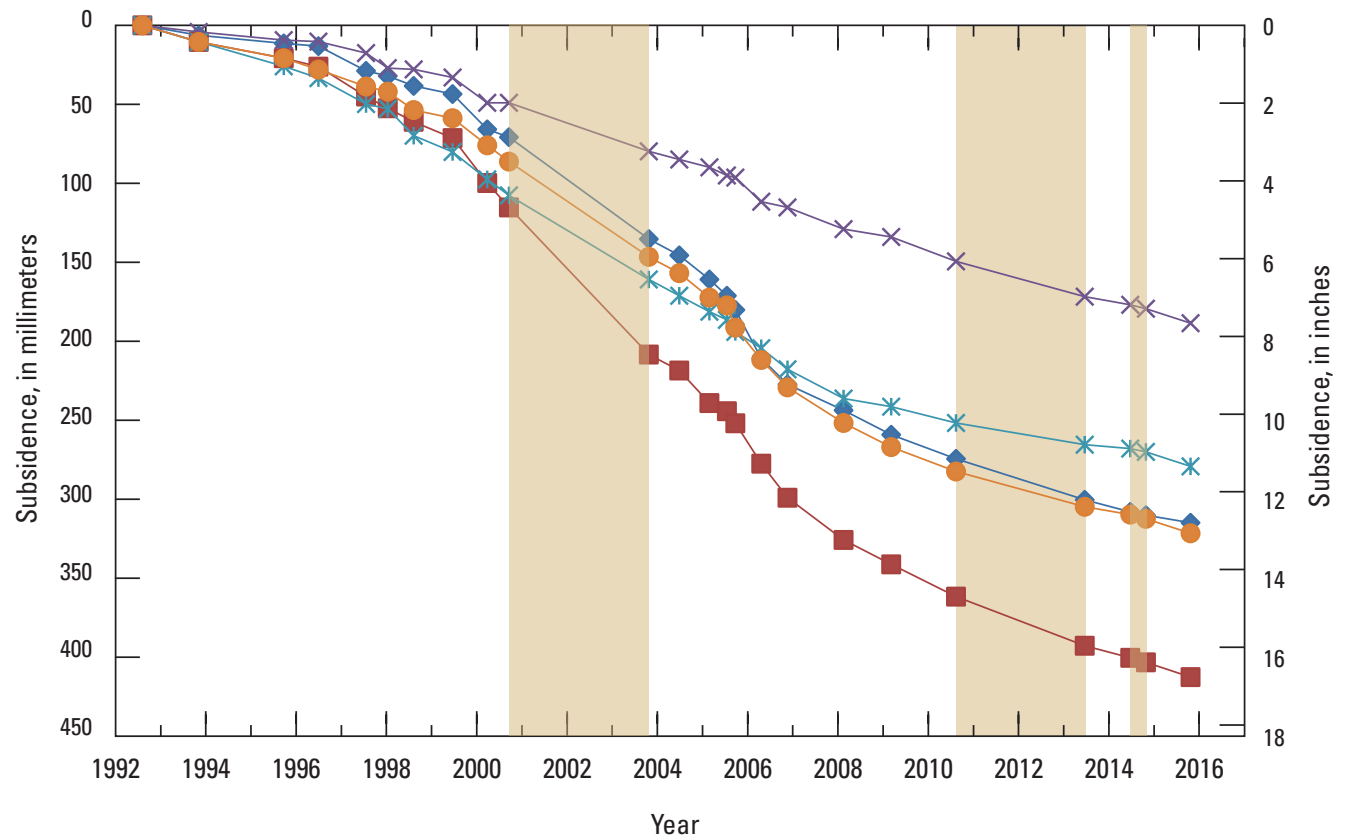

EXPLANATION

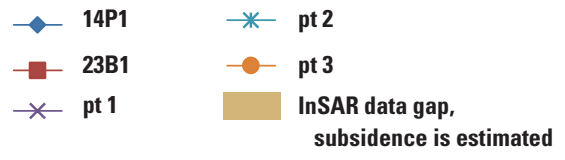

Figure 23. Interferogram-derived subsidence time-series data for five selected locations, 1992 to 2014, Bicycle Basin, Fort Irwin National Training Center, California.

\section{Surface Geophysical Surveys}

\section{Subsurface Imaging Near the Main-Runway Crack}

Results from the 2008 survey using the highest resolution, 1-m spacing (fig. 24A), show that the crack extended to a depth of at least $5 \mathrm{~m}(16 \mathrm{ft})$ and that there could be another feature approximately $20 \mathrm{~m}(65 \mathrm{ft})$ to the northeast for which there was no surface expression. Despite the fact that the playa soils are extremely conductive (resistivity less than $5 \Omega-\mathrm{m}$ ), results from the highest resolution survey indicated that subtle differences could be detected, such as the air-filled void space in the center of the crack zone presenting as a slightly more resistive vertical feature compared to the surrounding soil. The soil immediately surrounding the crack plane at depth showed as a less resistive feature relative to the surrounding soil. The less resistive feature was expected because of enhanced infiltration through the crack during periods of playa inundation, effectively increasing the localized soil moisture. This feature was still apparent in the survey using 2-m electrode spacing (fig. 24B) but could not be resolved in the deeper survey that required coarser electrode spacing (fig. 24C). Results from the multiple surveys demonstrated that it was difficult to effectively image features

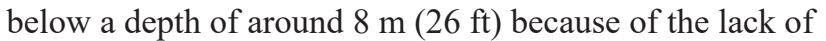
resistivity contrast in this highly conductive environment. Thus, the ERT results were inconclusive with respect to the characterization of the main-runway crack either as an Earth fissure or as the initial development of a macropolygon structure from giant desiccation cracking.

The 2017 survey results of the main-runway crack area using 2-m spacing (fig. 24D) were similar to the results from 2008. Imaging showed a vertical break at the crack (48-56 m, or $157-184 \mathrm{ft}$, along the survey line) where a relatively higher resistivity area was interpreted to be an air-filled cavity surrounded by slightly lower resistivity soil owing to enhanced soil moisture. It is also noteworthy that another vertical break was imaged to the northeast of the main-runway crack (90-98 m, or 295-322 ft, along survey line), similar to the results observed in 2008 (fig. 24B). The feature observed in the 2008 survey was roughly $20 \mathrm{~m}(65 \mathrm{ft})$ to the northeast of the crack, but it was around $40 \mathrm{~m}(131 \mathrm{ft})$ northeast of the crack in the 2017 survey. There were no observable surface expressions on the playa to explain these features; thus, it is unclear whether the features observed in the ERT imaging corresponded to one or more new features of potential concern for ground failures. 

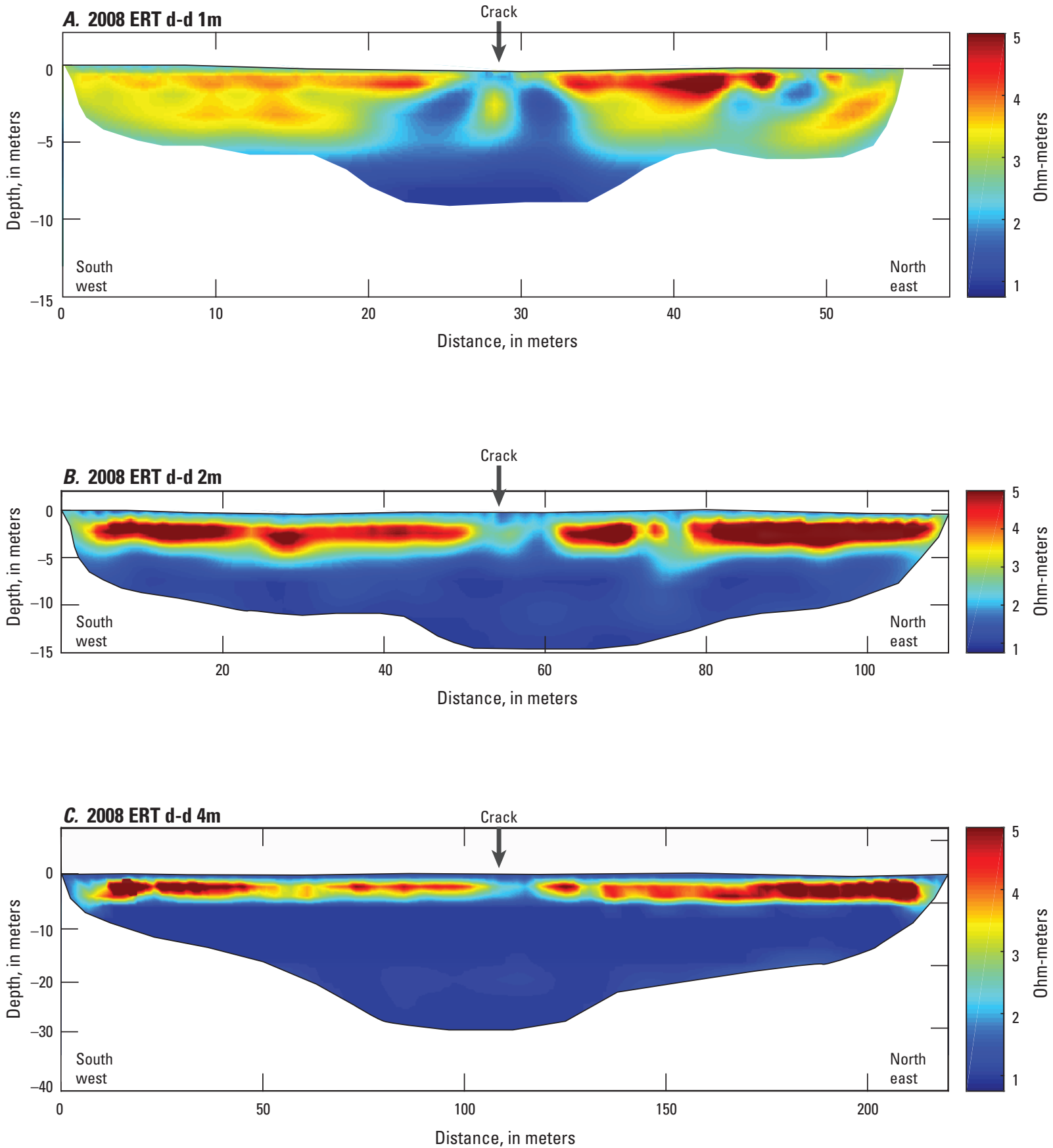

EXPLANATION

d-d $1 \mathrm{~m}=$ dipole-dipole at 1-meter spacing $\mathrm{d}-\mathrm{d} \mathbf{2 m}=$ dipole-dipole at 2-meter spacing d-d 4m=dipole-dipole at 4-meter spacing

Figure 24. Electrical resistivity tomography (ERT) survey results for the area around the main-runway crack, by year, and electrode spacing (location of survey line shown on fig. 8A), Bicycle Basin, Fort Irwin National Training Center, California: A, 2008 at 1-meter (m) increments perpendicular to crack; $B, 2008$ at 2-m increments perpendicular to crack; $C, 2008$ at 4-m increments perpendicular to crack; $D, 2017$ at 2-m increments for a survey line west of the 2008 line; $E, 2017$ at 2-m increments in the area of potential concern, south of the main-runway crack; and F, 2017 at 2-m increments in the giant desiccation crack area. 

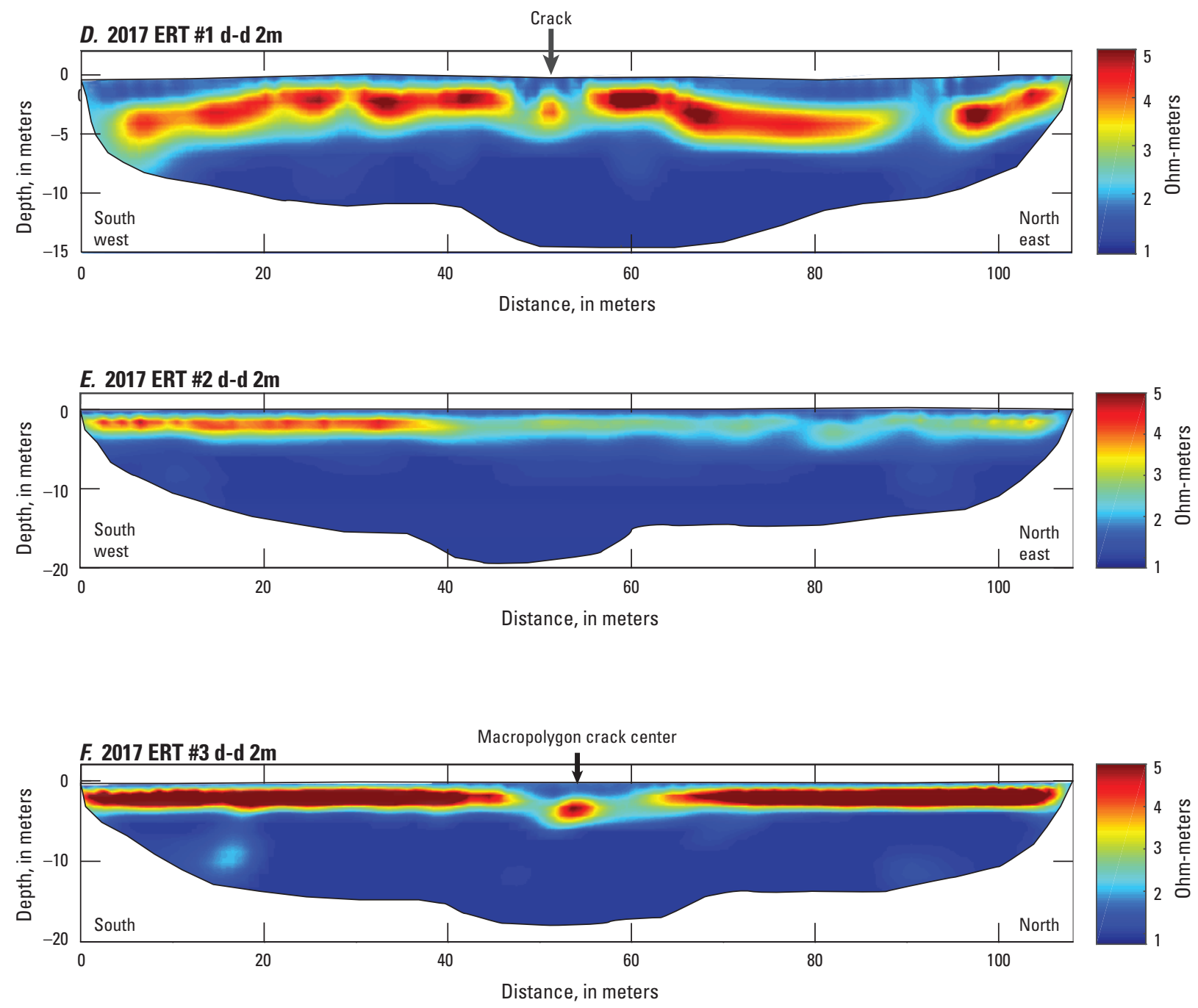

EXPLANATION

$\mathrm{d}-\mathrm{d} \mathbf{1 m}=$ dipole-dipole at 1-meter spacing $\mathrm{d}-\mathrm{d} \mathbf{2 m}=$ dipole-dipole at 2-meter spacing $\mathrm{d}-\mathrm{d}$ 4m=dipole-dipole at 4-meter spacing

Figure 24. - Continued 
The effectiveness of ERT surveying for detecting subsurface voids or other important features was evaluated by doing surveys of a potential area of concern, south of the main-runway crack (fig. $8 A ; 24 E$ ) and across a giant desiccation crack associated with a macropolygon feature (fig. 24F). Although ERT could clearly detect cracking features in the macropolygon area that appeared similar to the main-runway crack area (compare fig. $24 F$ with figs. $24 B, D$ ), it was not evident that we could confidently image any critical features that did not already have an expression at land surface. The ERT imaging at Bicycle Lake playa was limited by the entire shallow subsurface having extremely low resistivity (less than $5 \Omega \mathrm{m}$ ), with contrasts that were near the threshold of detection.

\section{Electromagnetic Induction Mapping for Ground Failures Assessment and Monitoring}

The EMI surveys were first done in Bicycle Basin in 2008. The objective of those surveys was to evaluate the effectiveness of the technique for identifying anomalies associated with known features of concern (that is, surface crack and macropolygon features) and for identifying areas of possible subsurface voids otherwise undetectable from visual inspection or remotely sensed imagery. Three separate parts of the basin were surveyed: (1) the area surrounding the main-runway crack, (2) the southern part of the playa where macropolygon features are prevalent, and (3) the toe of alluvial fans where subsidence was greatest at that time, based on the early analysis of InSAR data.

Results showed that the EMI technique was effective at revealing anomalous features (on the order of $100 \mathrm{mS} / \mathrm{m} \mathrm{ECa}$ above the background value) correlated with the features of concern in the playa area. The main-runway crack, numerous "healed" macropolygon features, and scattered sink-like depressions were all clearly identified as positive anomalies reflecting that these areas function as sinks for enhanced infiltration on the playa during periods of inundation, thus creating higher localized soil-moisture levels relative to the surrounding soil. In the main-runway crack area, this increase in soil moisture due to focused localized infiltration appeared to override the effect of subsurface void space in which the air portion of the void effectively reduces the ECa signal or, conversely, increases the electrical resistivity, as was observed in the ERT survey.

The EMI results across the sandy alluvial soils in the maximum subsidence area did not indicate any meaningful anomalies against an average background ECa of around $20 \mathrm{mS} / \mathrm{m}$. Despite this result for the alluvial area of the basin, the effectiveness of using EMI in the playa area indicated the technique is likely to be an effective approach for monitoring potential ground failures and areas of concern by mapping of positive anomalies.
Based on the results from the 2008 surveys, a pilot monitoring program for land-surface deformation was initiated in 2014 in which repeat EMI surveys covering the entire playa region were done on a regular basis. The goal of this program was to attempt to identify any potential emerging land-surface deformation features at the playa airstrip and to monitor the changes in existing subsurface features of concern. Mapping based on time-lapse EMI surveys could be able to help guide cone-penetration testing of the airstrip runways. Cone-penetrometer testing allows for subsurface site characterization of discrete stratigraphic horizons, discontinuous layers, and void spaces. Combining these methods is likely an effective approach for monitoring and evaluating the potential risk of cracking features to aircraft operations at the Bicycle Lake airstrip.

EMI surveys were completed spanning the entire Bicycle Lake playa in December 2014 (fig. 25A) and again in December 2015 (fig. 25B). Interpretation of the EMI results from 2014 revealed an area of concern for potential ground failures on the northeast part of the main runway for which there was no expression of cracking or sink-like depressions at land surface (figs. 25A, B). The two surface cracks that have developed since 2005 are outlined in black rectangles in figure 25 . The area of concern is approximately $150 \mathrm{~m}$ southwest of the main-runway crack. Comparison to the EMI survey results from 2008 confirmed that this area of concern formed between 2008 and 2014 (fig. 26). The repeat-survey results from 2015 indicated that this area remains a potential concern along the main-airstrip runway, but it appeared relatively stable during the 2014-15 period.

Difference mapping from the time-lapse surveys indicated that the main-runway crack area was relatively stable during the 2014-15 period (fig. 27). The only notable changes during the 2014-15 period (that is, beyond $50 \mathrm{mS} / \mathrm{m}$ ) appeared to be in a few areas next to the outer berm of the main runway, but on the southeast side, away from the active runway. Although there were a number of localized, discrete areas of change noted along the main runway in the difference map, the regular cycling of positive and negative changes and the localization of these data at the berm indicated that this result was simply an artifact of interpolation of the discrete survey data. This was clear when comparing the difference map results with the discrete survey-line data in detail around the main-runway crack area (fig. 28A), where the pattern of anomalies in the difference map arose from the inherently spikey response over berms combined with the variability in discrete data sampling between the surveys in 2014 (fig. 28B) and 2015 (fig. 28C). The differences noted earlier, southeast of the main-runway berm, did not appear to be an interpolation artifact and were interpreted as arising from localized changes in the shallow soil moisture in these areas. In particular, the predominantly negative differences observed in the 2014-15 period indicated drying of the soil in these areas and did not have the sharp, positive response that is characteristic of cracking features. 


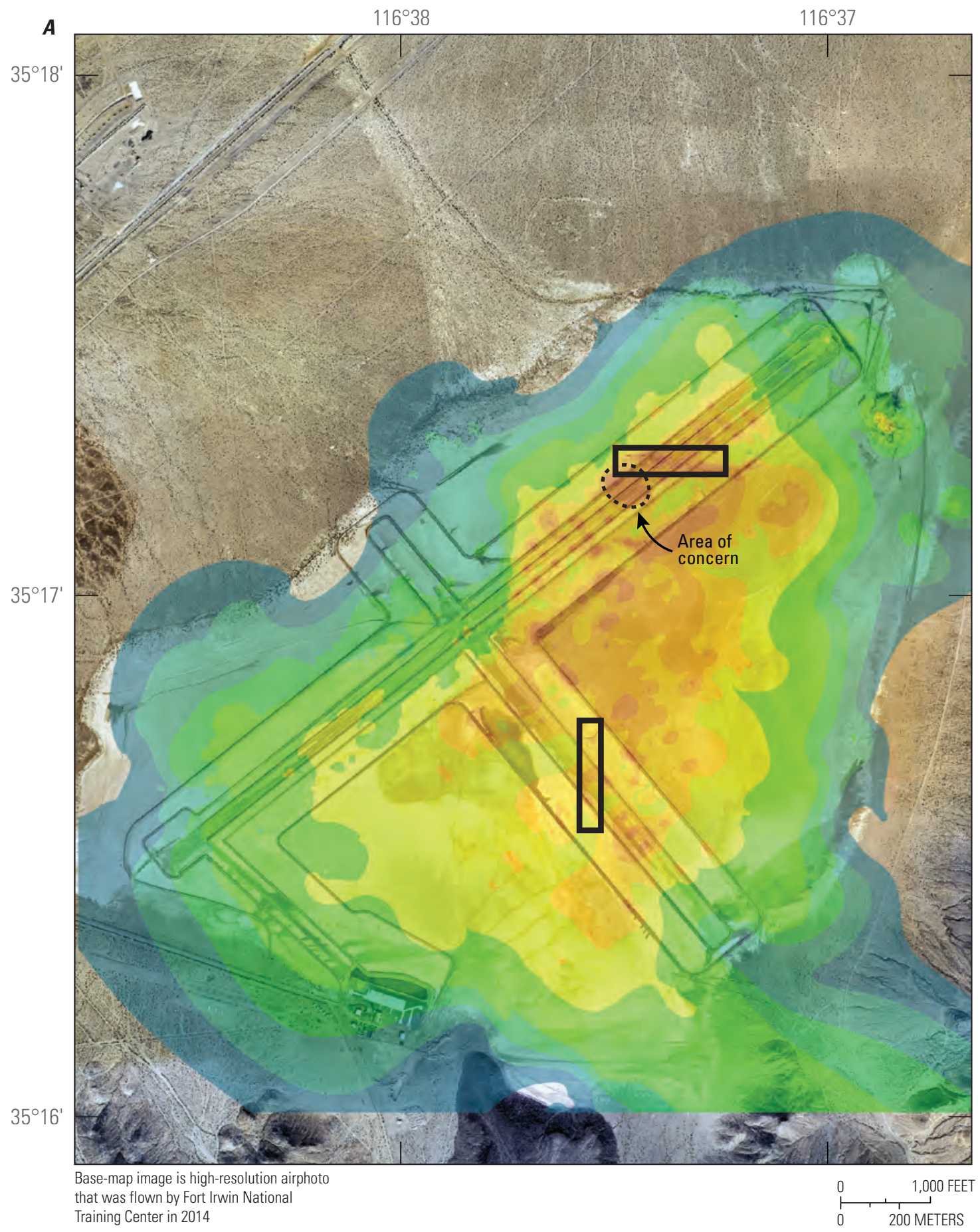

EXPLANATION

2014 Electromagnetic induction, in 18 kilohertz Apparent electrical conductivity (ECa), in millisiemens per meter

\begin{tabular}{|c|c|c|c|c|}
\hline $0-25$ & 51-75 & $101-150$ & $201-250$ & $301-400$ \\
\hline 26-50 & $76-100$ & $151-200$ & $251-300$ & $401-500$ \\
\hline
\end{tabular}

Figure 25. Apparent electrical conductivity (ECa, in millisiemens per meter) across the Bicycle Lake airstrip region, with known ground failures of two surface cracks that have formed since 2005 outlined in black, based on results from electromagnetic induction surveys in Bicycle Basin, Fort Irwin National Training Center, California, at 18 kilohertz operating frequency for $A$, December 2014; and $B$, December 2015. 


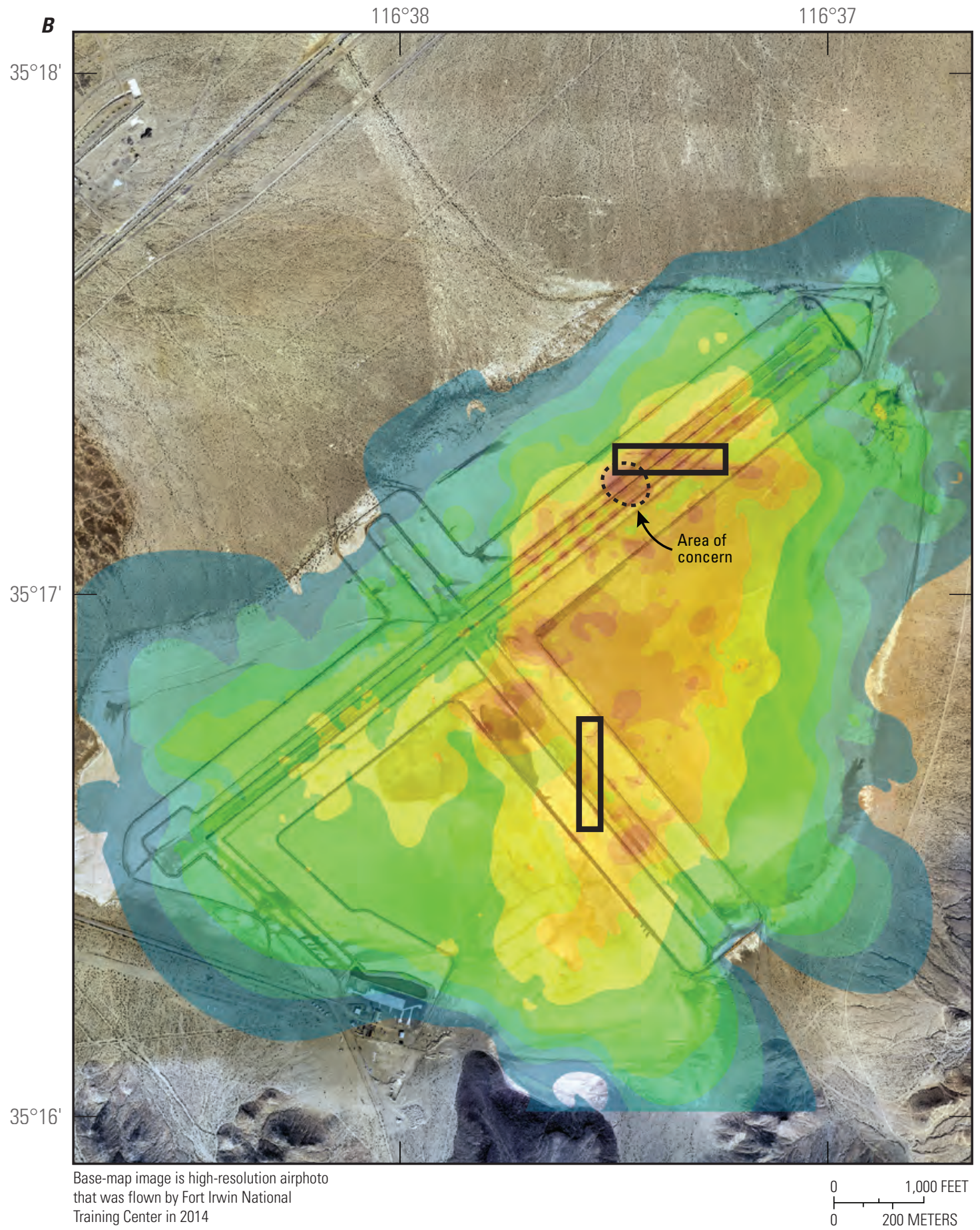

EXPLANATION

2015 Electromagnetic induction, in 18 kilohertz Apparent electrical conductivity ( $\mathrm{ECa})$, in millisiemens per meter

\begin{tabular}{|c|c|c|c|c|}
\hline $0-25$ & $51-75$ & $101-150$ & $201-250$ & $301-400$ \\
\hline $26-50$ & 76-100 & $151-200$ & $251-300$ & $401-500$ \\
\hline
\end{tabular}

Figure 25. -Continued 


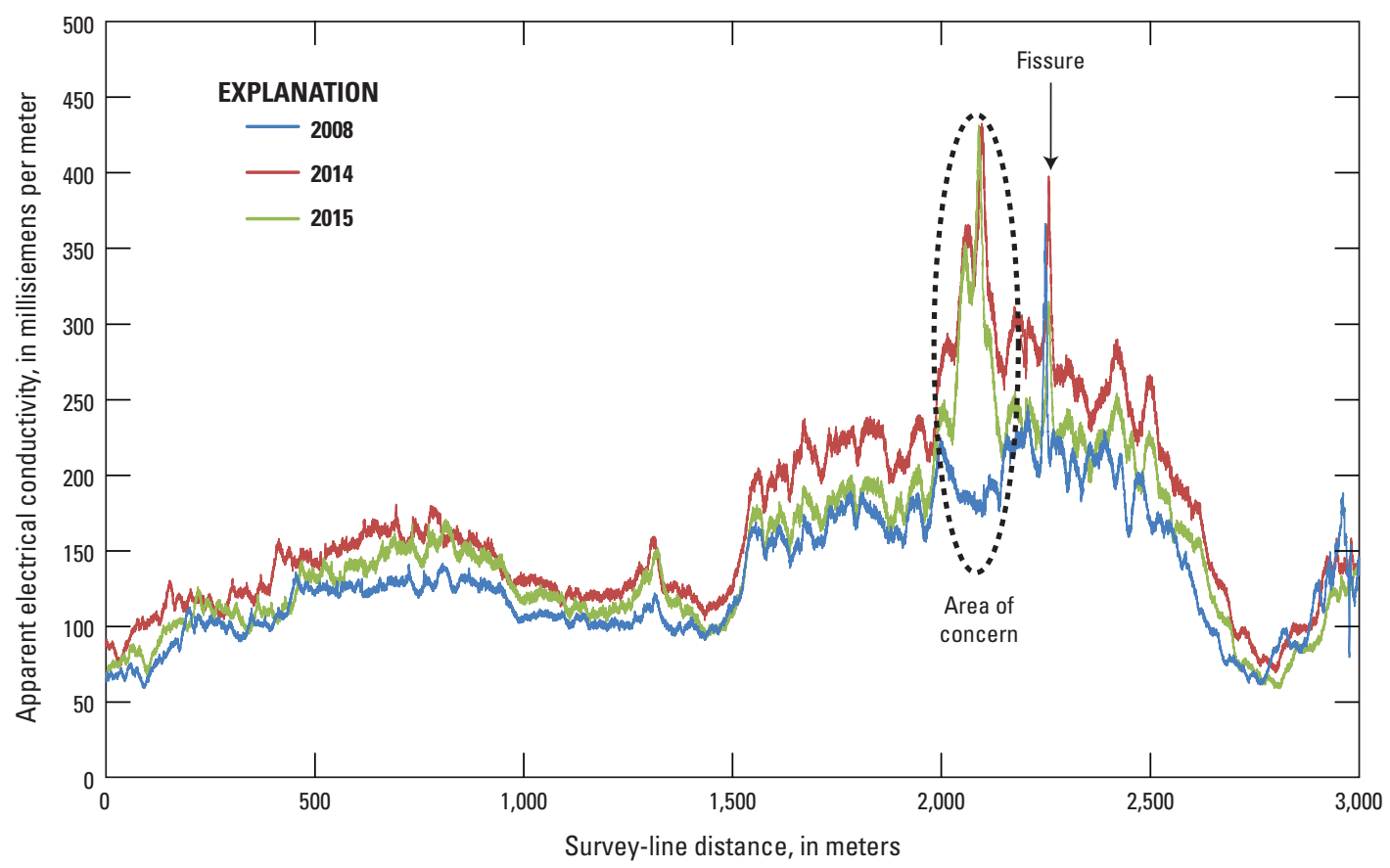

Figure 26. Electromagnetic induction surveys for the main runway (location shown on fig. 8A) from 2008, 2014, and 2015, Bicycle Basin, Fort Irwin National Training Center, California. Notable departure in the later surveys at around a line distance of 2,100 meters indicates an area of concern for potential subsurface voids undetectable by visual inspection of the ground surface.

\section{Hypothesis Testing With Numerical Experiments}

Model results from simulations of water-table decline at depth suggested that it could be difficult for desaturation of the capillary fringe zone to effectively transmit stresses through the overlying soil large enough to cause cracking up to the surface (figs. 29, 30, 31). This brings into question the hypothesis that water-table decline at depth is the mechanism driving the development of giant desiccation cracks and macropolygon structures. The analysis of macropolygon features in Death Valley by Antrett and others (2012) also challenged this hypothesis to some extent, given that active development of macropolygon features was in an area where the water table appeared to be at a shallow depth of just a few meters or less.
Results from the simulations of the desiccation process in the presence of a large, regional tectonically induced stress field (fig. 32) supported the hypothesis that this could indeed be a key factor for the formation of macropolygons rather than desiccation mud cracks of typical size (that is, polygons which are centimeters in diameter). The large, initial tensile strain ( 0.01 percent) simulated in the model adds stress beyond the desiccation shrinkage process, effectively creating larger cracks perpendicular to the direction of maximum tension. Cracks align nearly in parallel, and the spacing between cracks increases. This would result in larger polygonal features on the surface than those resulting from desiccation alone. A crack at depth, such as the one on the bottom corner of figure 32, could potentially propagate to more shallow layers, given the combination of initial tensile stresses and desiccation. 


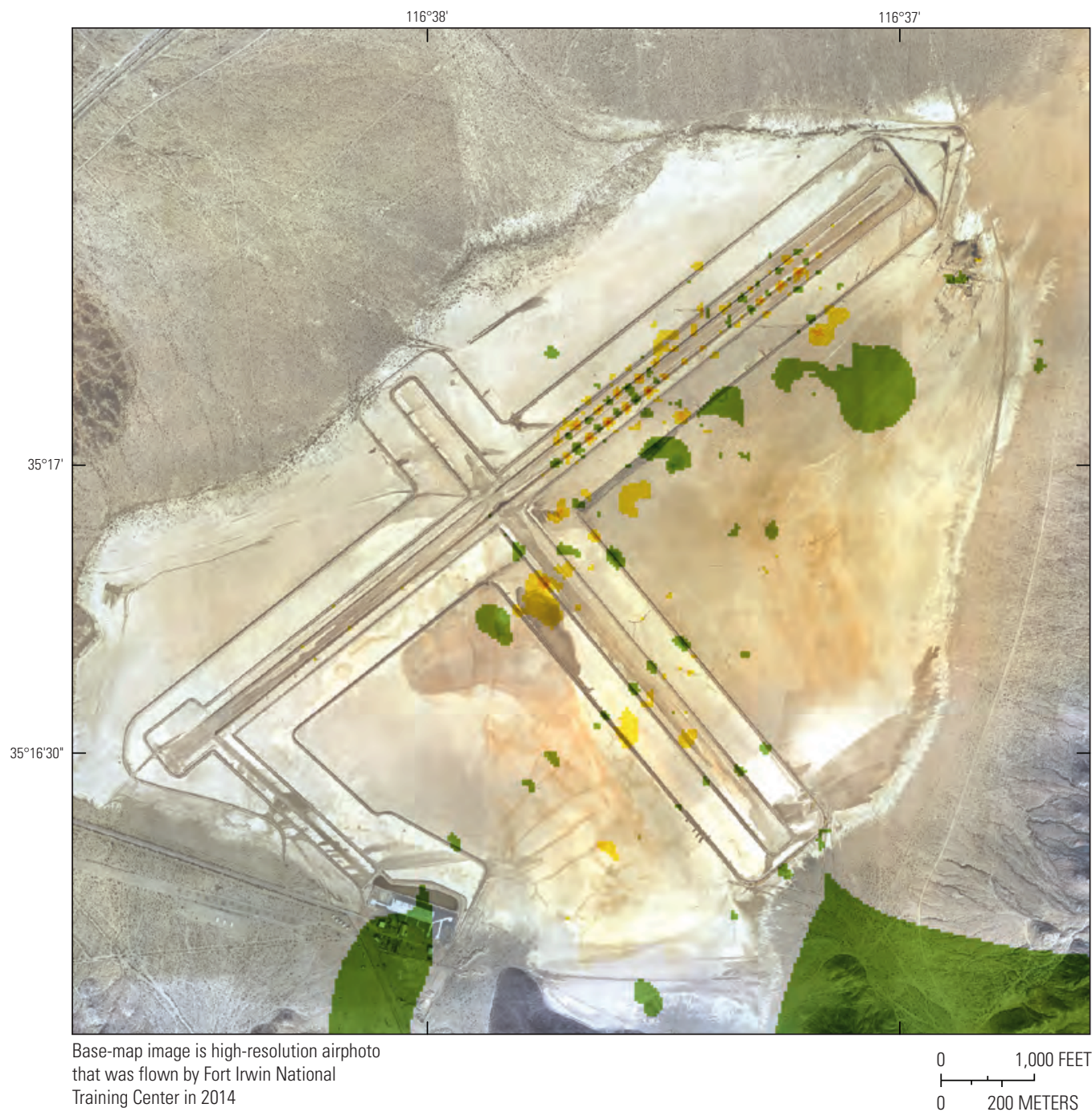

\section{EXPLANATION}

Time-lapse surveys, 2014-15

Difference in apparent electrical conductivity (ECa), in millisiemens per meter

\begin{tabular}{|c|c|c|c|c|}
\hline-200 to -151 & -100 to -51 & 0 to 50 & 101 to 150 & 201 to 250 \\
\hline-150 to -101 & -50 to 0 & 51 to 100 & & 251 to 300 \\
\hline
\end{tabular}

Figure 27. Electromagnetic induction time-lapse surveys of Bicycle Basin, Fort Irwin National Training Center, California, for 2014-15. 


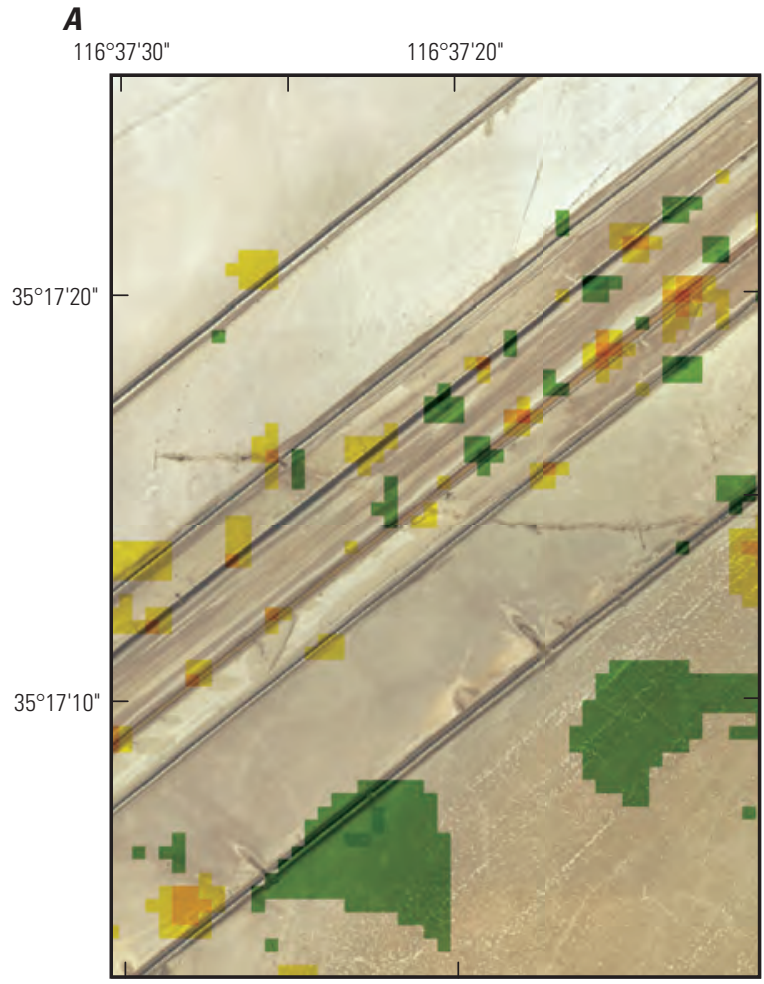

\section{EXPLANATION}

Time-lapse surveys, 2014-15

Difference in apparent electrical conductivity (ECa), in millisiemens per meter

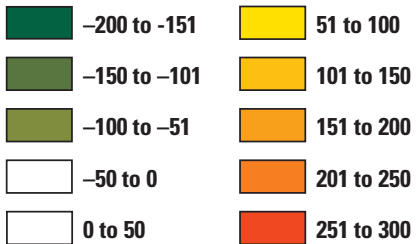

2014-15 Electromagnetic induction, in 18 kilohertz Apparent electrical conductivity (ECa), in millisiemens per meter

\begin{tabular}{|c|}
\hline 101-150 \\
\hline 151-200 \\
\hline $201-250$ \\
\hline
\end{tabular}

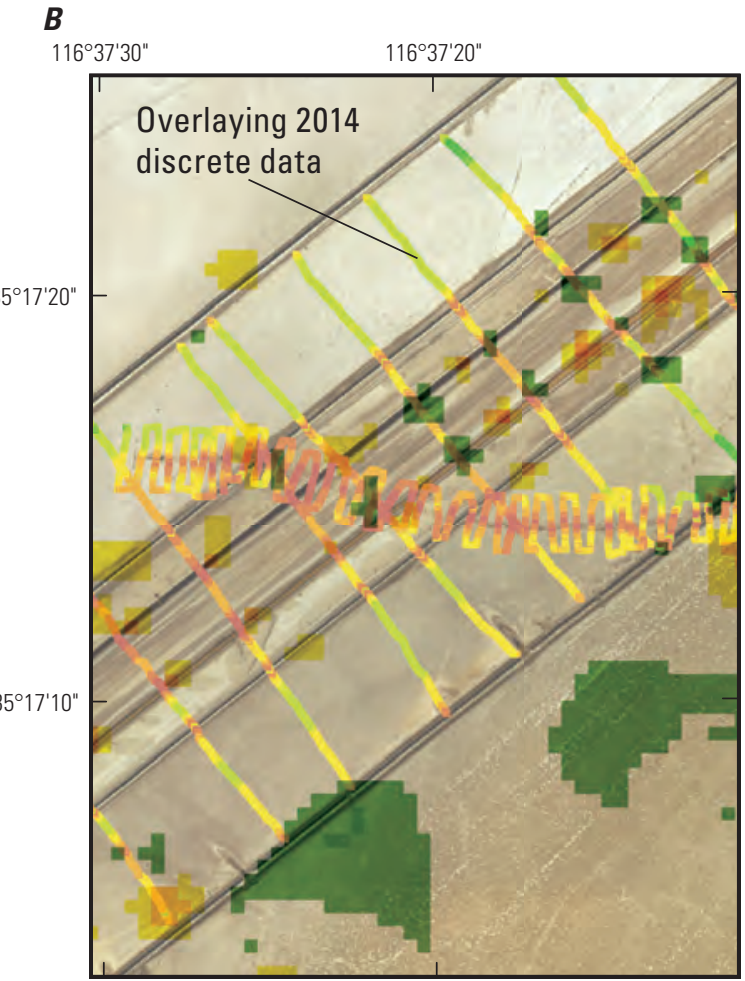

\section{C}
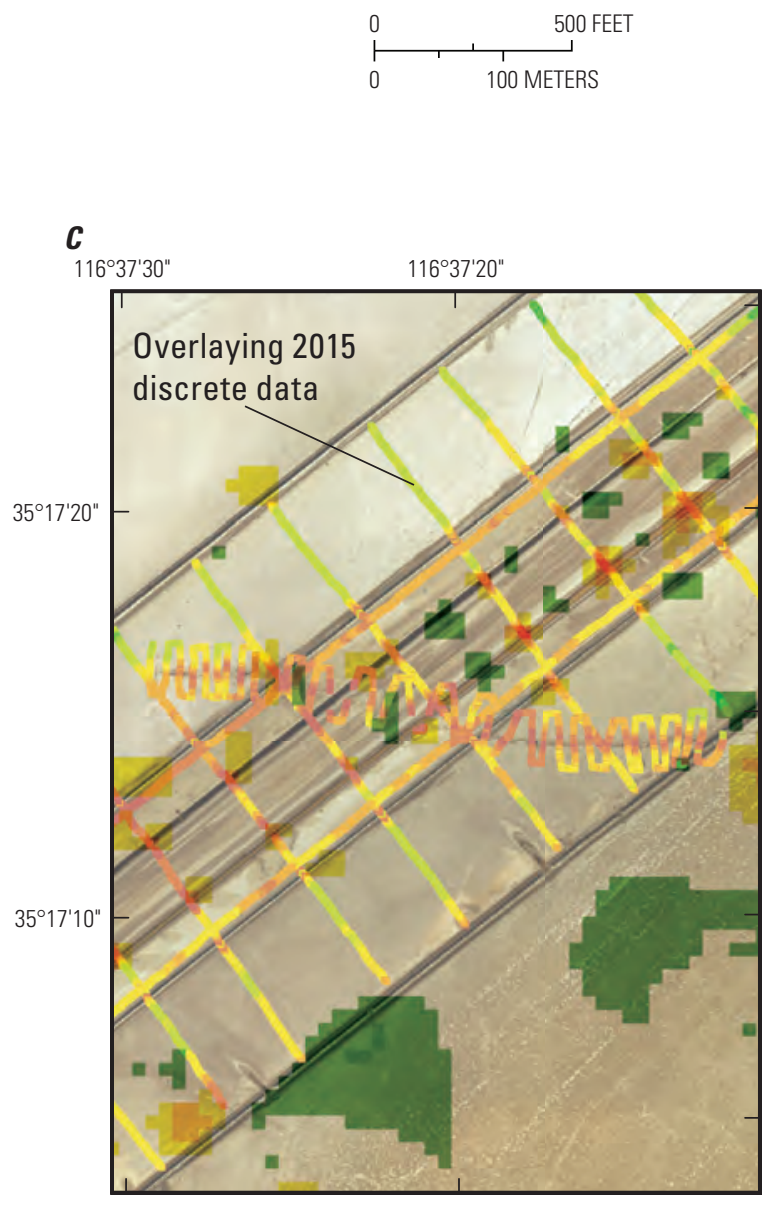

Base-map image is high-resolution airphoto

that was flown by Fort Irwin National

Training Center in 2014

Figure 28. Electromagnetic induction time-lapse surveys in Bicycle Basin, Fort Irwin National Training Center, California, indicate the main-runway crack was stable during the 2014-15 period: $A$, difference map (note pattern of anomalies that arise from the inherently spikey response over berms combined with the variability in discrete data sampling between survey lines); $B$, difference map overlain with 2014 discrete data; and C, difference map overlain with 2015 discrete data. 

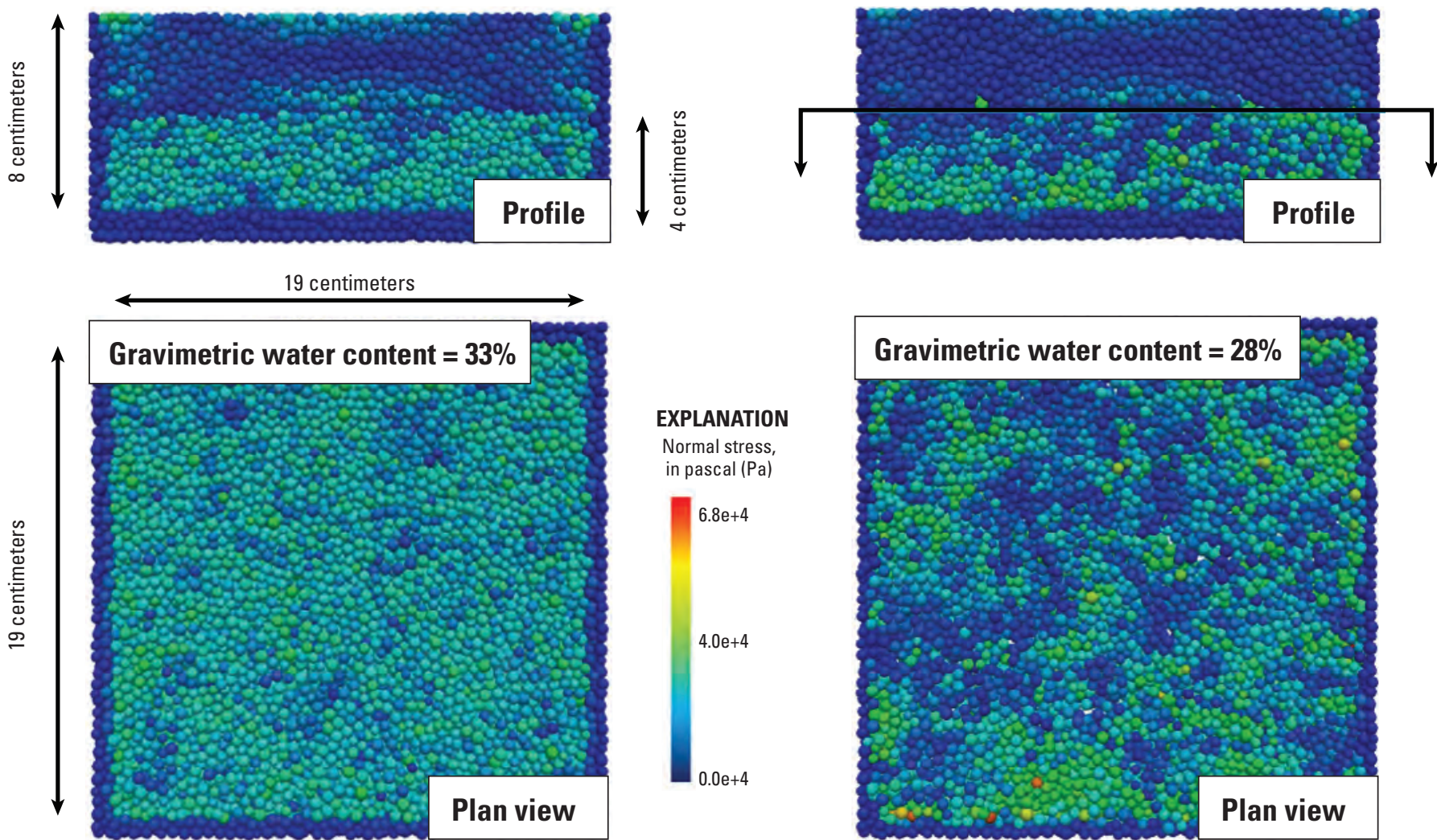

Figure 29. Discrete element method model results for particle-stress accumulation from simulating shallow water-table drawdown.

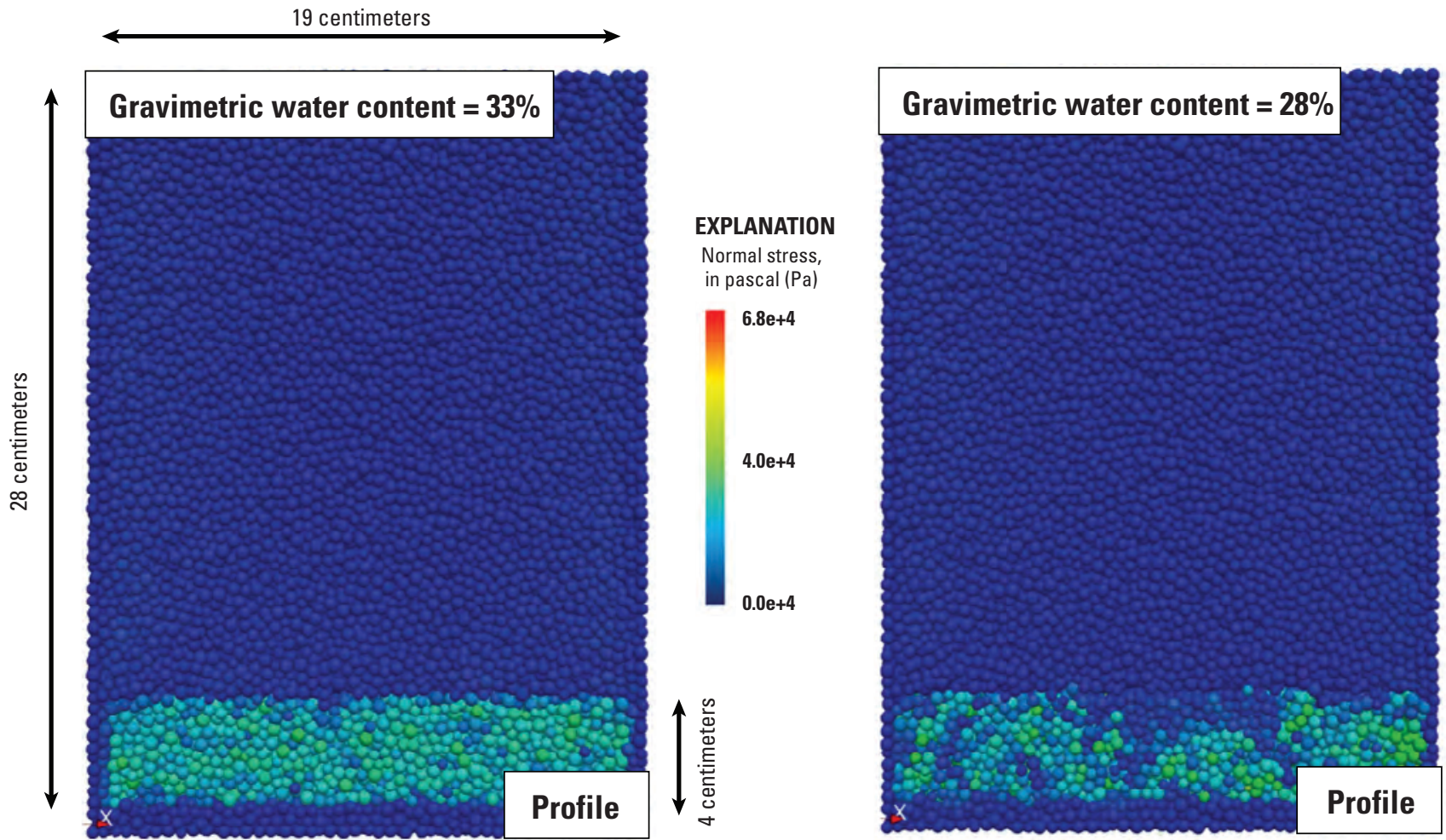

Figure 30. Discrete Element Method model results for particle-stress accumulation from simulating deep water-table drawdown. $[\%$, percent $]$ 

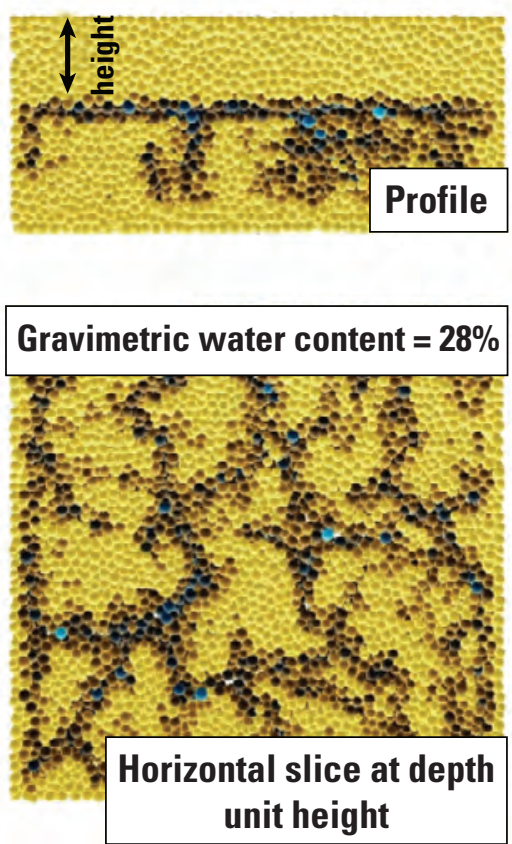
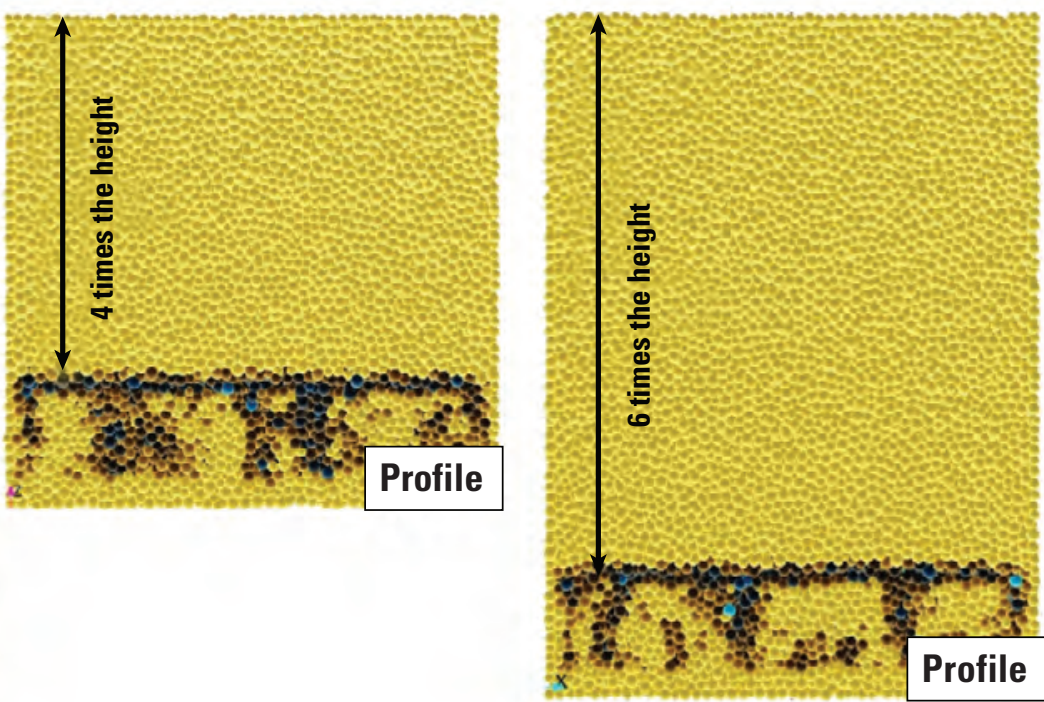

Figure 31. Discrete Element Method model results for crack-propagation simulation (particle bond breaking) indicates cracking induced from desiccation volumetric shrinkage in the capillary-fringe zone above a declining water table is unable to propagate upward to the shallow, overlying soil. Darker colors indicate an increase in broken bonds between particles as stress builds from volumetric shrinkage due to desiccation.
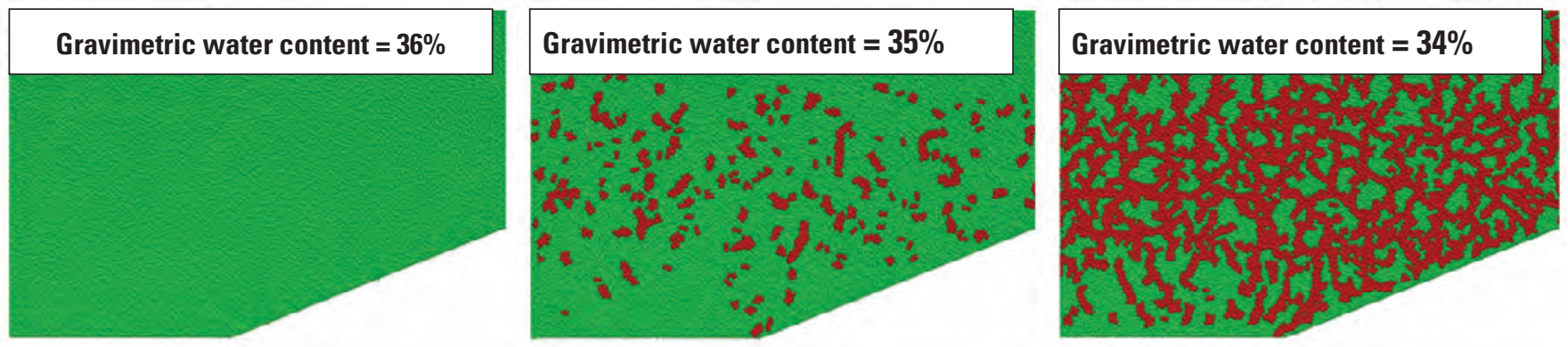

Tensile strain $(\varepsilon)=0.01 \%$
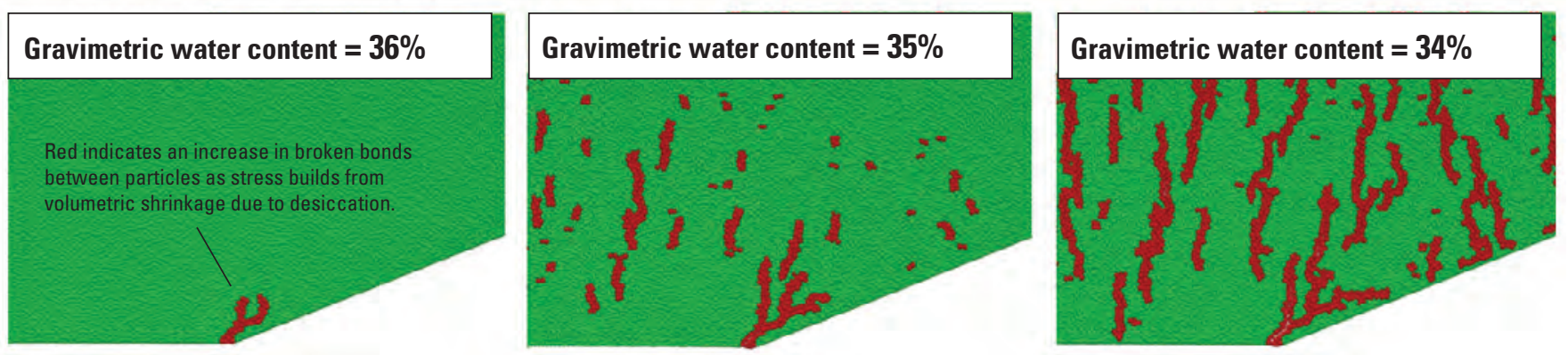

Figure 32. Discrete element method model results for crack formation (red) from the desiccation process alone (upper row) compared to the combined processes of desiccation and tensile strain arising from a large, regional tectonic-stress field (bottom row). Figures are profile views corresponding to the lower right part of the two-dimensional, vertical cross section shown in figure 12. [\%, percent] 


\section{Summary and Conclusions}

The first large surface crack formed across the main runway of the Bicycle Lake airstrip in 2005-06. Numerous sink-like depressions were also discovered in the northeastern part of the playa following an exceptionally long period of lake-like conditions in the spring of 2005. The large, single crack across the main runway was hypothesized to be an Earth fissure - tensile failures that result from differential compaction in the subsurface related to aquifer depletion. Another large crack formed on the playa in 2013, following another period of lake-like conditions. This crack was notably different than the 2005-06 crack in that it had a bifurcated morphology. The 2013 crack formed in the area of the playa that has notable patterned ground of macropolygon structure (distinct sediment polygons similar to mud-crack polygons, but on the scale of hundreds of meters in diameter) roughly a kilometer away from the 2005-06 crack. Aerial photography showed that macropolygon structure associated with giant desiccation cracks was a persistent feature of the southern part of Bicycle Lake playa, dating back to at least the 1940s. In this study, the U.S. Geological Survey, in cooperation with the U.S. Army Fort Irwin NTC, evaluated the role of shallow desiccation processes compared to deeper differential compaction in driving the recent surface cracking at Bicycle Lake playa.

Groundwater pumping in the Bicycle Basin began in the 1960s, and pumpage has generally increased since the 1990s. The extent and effect of groundwater-level declines and land-surface deformation in Bicycle Basin were evaluated after a large crack formed at the surface of Bicycle Lake playa in the area used as an aircraft runway. Multiple approaches were used including measurements of water levels from December 2007 to June 2017, land surveys across the playa area, remotely sensed ground displacements with interferometric synthetic aperture radar (InSAR), geophysical surveys of the playa area, and numerical experiments to test hypotheses regarding soil mechanical processes. A specific objective of this study was to evaluate the recent development of ground failures in the form of large surface cracks that pose a hazard to aircraft operations on the Bicycle Lake playa airstrip.

Water levels have declined throughout Bicycle Basin since pumping began in the mid-1960s. Pumping has resulted in more than 27 meters ( $\mathrm{m}$; 90 feet, or ft) of water-level declines from 1990 to 2017 in wells north of the Bicycle Lake playa, where InSAR data showed more than 400 millimeters (mm; 16 inches, or in.) of subsidence between 1993 and 2015. Water levels measured during 2017 were lowest in the central and northeastern part of the basin, centered in the area of maximum subsidence. From December 2007 to June 2017, water levels recovered during periods of less pumping, which were generally during the fall or winter seasons. Pumping was not stopped for long enough to allow water levels to recover to previous water levels, however. Although the overall long-term trend in water levels during this time was generally downward, water levels in the main pumping zone showed some recovery that correlated with a reduction in pumping during 2013 and 2015-16, interrupted by period of increased pumping during 2014.

Results of leveling showed differential subsidence along a transect across the playa and into the area of maximum subsidence between 2009-16, with less subsidence south of the 2005-06 main-runway crack between monuments BL2 and BLF4; more subsidence north of the 2005-06 crack between BLF4 and BL7; and the greatest subsidence gradient, from BL7 to BL8 before leveling off between BL8 and BLA4. The subsidence rate was relatively steady from 2009 to 2014: 1.3 millimeters per month $(\mathrm{mm} / \mathrm{mo} ; 0.05$ inches per month, or in. $/ \mathrm{mo})$ from January 2009 to September 2010, $1.7 \mathrm{~mm} / \mathrm{mo}(0.07 \mathrm{in} . / \mathrm{mo})$ from September 2010 to April 2011, and $1.1 \mathrm{~mm} / \mathrm{mo}$ (0.04 in./mo) from April 2011 to October 2014. The subsidence rate slowed to $0.3 \mathrm{~mm} / \mathrm{mo}(0.01 \mathrm{in} . / \mathrm{mo})$ from October 2014 to November 2015 and to $0.46 \mathrm{~mm} / \mathrm{mo}(0.018 \mathrm{in} . / \mathrm{mo})$ from November 2015 to December 2016 in response to the decrease in pumping that began in 2013, the increase during 2014, and the decrease again during 2015-16.

Repeated tapex measurements from April 2009 to November 2017 across the main-runway crack indicated opening of the crack between monuments BLF3 and BLF4, but no notable change across BLF1 and BLF2 at the western terminus of the crack. The data collected showed relatively high variability in the 2014-17 set of measurements, likely as a result of instability from temperature effects. The overall trend of the data between BLF3 and BLF4 indicated that the crack widened approximately $13.7 \mathrm{~mm}(0.5 \mathrm{in}$.) between April 2009 and November 2017. This was concomitant with about $1.8 \mathrm{~m}$ (6 ft) of water-level decline during 2009-15 and $0.7 \mathrm{~m}(2.5 \mathrm{ft})$ of water-level rise during 2015-17 in the main pumped zone at monitoring site BLA4, in the area of greatest measured subsidence. During 2013 and 2015-16, pumping decreased in Bicycle Basin.

A baseline lidar survey of the main-runway crack was done in January 2009 to track the development of this feature. Vertical land-surface change was evaluated by comparing sequential surveys and creating a total change image for 2009-16. During the 95 months from January 2009 to December 2016, $0.5 \mathrm{~m}$ (19.7 in.) of subsidence was observed next to the subsidence pit in the crack along the western end of the scanned area, presumably due to erosion and backfilling during periods when the lake was inundated. Standing water in the crack along the eastern end of the scanned area prevented determining change for this part of the crack. Little to no change was observed in the crack in most of the scanned area. Time-series shaded-relief images showed the progression of changes in the crack from January 2009 to December 2016. During 2010, the crack appeared to be filling; then, it opened slightly in a few places during 2011. By 2014, a large sink-like depression had formed near the western end of the crack in the scanned area. The crack appeared to be filling again by 2016 . 
Volumetric changes of the crack were calculated between the sequential lidar surveys for the west and east ends of the crack using a fixed reference plane and the land surface for each survey. The change in crack volume due to subsidence or deposition was estimated by calculating the difference between surface to plane volumes in sequential lidar surveys. From 2009 to 2016, the cumulative volumetric change was an increase of 1.5 cubic meters $\left(\mathrm{m}^{3} ; 54\right.$ cubic feet, or $\left.\mathrm{ft}^{3}\right)$. This volume is a minimum estimate because the crack volume below the water surface in the pits was not included in the calculations.

Of the 103 interferograms of Bicycle Basin analyzed for this study, 90 showed land-surface elevation losses, or subsidence, in the area immediately north of Bicycle Playa, in the northwestern part of Bicycle Basin. The area affected by subsidence was about 6.7 square kilometers $\left(\mathrm{km}^{2}\right.$; 2.6 square miles, or $\mathrm{mi}^{2}$ ) and coincided with an area containing substantial clay deposits. Subsidence rates calculated from interferograms were variable. Rates during 1992-97 ranged from 0.7 to $2.7 \mathrm{~mm} / \mathrm{mo}(0.03-0.11 \mathrm{in} . / \mathrm{mo})$ and averaged $1.6 \mathrm{~mm} / \mathrm{mo}$ (0.06 in./mo); rates during 1998-2007 ranged from 1.0 to $4.4 \mathrm{~mm} / \mathrm{mo}(0.04-0.18 \mathrm{in} . / \mathrm{mo})$ and averaged $2.6 \mathrm{~mm} / \mathrm{mo}(0.11 \mathrm{in} . / \mathrm{mo})$, and rates during $2008-15$ ranged from 0.4 to $3.0 \mathrm{~mm} / \mathrm{mo}(0.01-0.12 \mathrm{in} . / \mathrm{mo})$ and averaged $1.5 \mathrm{~mm} / \mathrm{mo}(0.06 \mathrm{in} . / \mathrm{mo})$. These data indicated that subsidence rates during 1992-97 and 2008-15 were nearly half the rates during 1998-2007. Some of the interferograms showed distinct linear features at the outer edges of the subsidence area, indicating the presence of faults or abrupt changes in lithology that affect the extent of subsidence. Changes in the timing and total annual volume of groundwater withdrawal can affect the conditions that manifest as linear features in InSAR imagery. Model-inferred fault locations correlated with subsidence patterns noted in most interferograms used in this study and appeared, in part, to control the lateral extent of subsidence.

Subsurface imaging of the main-runway crack area was done with a series of electrical resistivity tomography (ERT) surveys in 2008 and in 2017. The 2008 surveys used different electrode spacing that allowed multiple two-dimensional, vertical slice images down to a depth of around $46 \mathrm{~m}(151 \mathrm{ft})$. A single deep structure penetrating to such a depth could indicate that the crack was indeed an Earth fissure resulting from differential compaction of the basin sediments, rather than the initial branch of a developing giant desiccation-crack network. Results from the surveys indicate that the crack could extend $5 \mathrm{~m}(16 \mathrm{ft})$ or more into the subsurface, but it is difficult to conclude because of the lack of resistivity contrast in this highly conductive environment. Thus, the imaging results from the 2008 ERT surveys were inconclusive with respect to the characterization of the main-runway crack either as an Earth fissure or as the initial part of a macropolygon structure forming from giant desiccation cracking. Results from the 2017 ERT survey across the main-runway crack were similar to the 2008 results. Although this could indicate only minor development of the feature during the 9 years between surveys, it is challenging to use ERT effectively in this environment because of the ubiquitously conductive sediments. Indeed, results from two additional ERT surveys in 2017 proved impossible to interpret beyond a depth of about $5 \mathrm{~m}$.

Electromagnetic induction (EMI) surveys were done in Bicycle Basin in 2008, 2014, and 2015 to evaluate the potential effectiveness of the technique as a tool to monitor land-surface deformation. Results showed that the technique was effective at revealing anomalous features (on the order of 100 millisiemens per meter apparent electrical conductivity above the background value) that correlated to the features of concern in the playa area, such as the main-runway crack, numerous "healed" macropolygon features, and scattered sink-like depressions. The EMI results from 2014 indicated an area of concern for potential ground failure on the northeast part of the main runway, approximately $150 \mathrm{~m}$ southwest of the main-runway crack, that showed no visible cracking or sink-like depressions at land surface. Comparison to the EMI survey results from 2008 confirmed that this feature developed between 2008 and 2014. The repeat survey results from 2015 confirmed that this area was still of concern as a potential ground failure on the main runway, but appeared relatively stable during the 2014-15 period. Difference mapping from the time-lapse surveys indicated that the main-runway crack area had also been relatively stable during the 2014-15 period. The only notable changes during this period appeared to be in several areas next to the outer berm of the main runway, but on the southeast side away from the runway. The predominantly negative differences observed during the 2014-15 period indicated drying of the near-surface sediments in these areas and did not have the sharp positive response characteristic of cracking features.

Results from numerical experiments simulating watertable decline at depth could indicate that it is somewhat unlikely that desaturation of the capillary-fringe zone would be able to transmit large enough stresses through the overlying soil to cause cracking at the land surface. Results from simulations of the desiccation process in the presence of a regional, tectonically induced stress field tended to support the hypothesis that the combined processes could be key to determining why macropolygons form in certain areas, such as Bicycle Lake playa. 
Considering the results from all the methods used in this study, it appears likely that the shallow desiccation process and deeper stresses driven by localized, differential compaction from pumping or by land-surface deformation from tectonic forces are both playing a role in the formation of cracking features at the Bicycle Lake playa airstrip. Time-lapse EMI surveys appeared to be an effective approach to monitoring these features, both in terms of evaluating the development of existing known ground failures and identifying areas of concern for which there are no visible indications of cracking or depressions at land surface. Mapping based on time-lapse EMI surveys could be able to help guide cone-penetration testing of the airstrip runways. Cone-penetrometer testing allows for subsurface site characterization of discrete stratigraphic horizons, discontinuous layers, and void spaces. Combining these methods is likely an effective approach for monitoring and evaluating the potential risk of cracking features to aircraft operations at the Bicycle Lake airstrip.

\section{References Cited}

Amarasiri, A., Kodikara, J., and Costa, S., 2011, Numerical modelling of desiccation cracking: International Journal for Numerical and Analytical Methods in Geomechanics, v. 35, p. 82-96, https://doi.org/10.1002/nag.894.

Amelung, F., Galloway, D.L., Bell, J.W., Zebker, H.A., and Laczniak, R.J., 1999, Sensing the ups and downs of Las Vegas-InSAR reveals structural control of land subsidence and aquifer-system deformation: Geology, v. 27 , no. 6, p. 483-486, https://doi.org/10.1130/00917613(1999)027<0483:STUADO>2.3.CO;2.

Antrett, P., Vackiner, A.A., Kukla, P., Klitzsch, N., and Stollhofen, H., 2012, Impact of arid surface megacracks on hydrocarbon reservoir properties: American Association of Petroleum Geologists Bulletin, v. 96, no. 7, p. 1279-1299.

Bedinger, M.S., and Harrill, J.R., 2012, Groundwater geology and hydrology of Death Valley National Park, California and Nevada: Fort Collins, Colo., National Park Service Natural Resource Technical Report NPS/ NRSS/WRD/NRTR—2012/652, 117 p., available at https://irma.nps.gov/DataStore/DownloadFile/461112.

Bell, J.W., 1981, Subsidence in Las Vegas Valley: Nevada Bureau of Mines and Geology Bulletin 95, 83 p., 1 pl., scale 1:62,500.

Bell, J.W., and Price, J.G., 1991, Subsidence in Las Vegas Valley, 1980-91-Final project report: Nevada Bureau of Mines and Geology, Open-File Report 93-4, 10 sect., 9 pls., scale $1: 62,500$.
Burbey, T.J., 2010, Mechanisms for earth fissure formation in heavily pumped basin, in land subsidence, associated hazards and the role of natural resources development: International Association of Hydrological Sciences Publication 339, p. 3-8.

California Interagency Watershed Mapping Committee, 2004, The California interagency watershed map of 1999 (updated May 2004): California Department of Fish and Game.

Carpenter, M.C., 1993, Earth-fissure movements associated with fluctuations in ground-water levels near the Picacho Mountains, south-central Arizona, 1980-84: U.S. Geological Survey Professional Paper 497-H, https://doi.org/10.3133/pp497H.

C.F. Hostrup and Associates, 1955, Water resources survey, Camp Irwin, California: Los Angeles, Calif., U.S. Army Corps of Engineers, Office of the District Engineer, 109 p.

Cundall, P.A., and Strack, O.D.L., 1979, A discrete numerical model for granular assemblies: Géotechnique, v. 29, no.1, p. 47-65, https://doi.org/10.1680/geot.1979.29.1.47.

Dang, H.K., and Meguid, M.A., 2010, Algorithm to generate a discrete element specimen with predefined properties: International Journal of Geomechanics, v. 10, no. 2, p. 85-91, https://doi.org/10.1061/(ASCE)GM.19435622.0000028 .

Daniels, F., and Alberty, R.A., 1966, Physical chemistry (3d ed.): New York, John Wiley and Sons, Inc., 639 p.

Densmore, J.N., and Londquist, C.J., 1997, Ground-water hydrology and water quality of Irwin Basin at Fort Irwin National Training Center, California: U.S. Geological Survey Water-Resources Investigations Report 97-4092, 159 p., https://doi.org/10.3133/wri974092.

Densmore, J.N., Woolfenden, L.R., Rewis, D.L., Martin, P.M., Sneed, M., Ellett, K.M., Solt, M., and Miller, D.M., 2018, Geohydrology, geochemistry, and numerical simulation of groundwater flow and land subsidence in the Bicycle Basin, Fort Irwin National Training Center, California: U.S. Geological Survey Scientific Investigations Report 2018-5067, 176 p., https://doi.org/10.3133/sir20185067.

Dintaman, C.R., Ellett, K.M., and Densmore, J.N., 2019, Electromagnetic induction (EMI) geophysical data collected at the Bicycle Basin, U.S. Army Fort Irwin National Training Center, California: U.S. Geological Survey data release, https://doi.org/10.5066/P9AW3J1W.

EarthInfo, Inc., 1995, National Climatic Data Center summary of the day-West 1994: Boulder, Colo., EarthInfo, Inc., CD-ROM. 
EarthInfo, Inc., 2000, EarthInfo CD2 reference manualfor all EarthInfo CD-ROM databases: Boulder, Colo., EarthInfo, Inc.

El Maarry, M.R., Kodikara, J., Wijessoriya, S., Markiewicz, W.J., and Thomas, N., 2012, Desiccation mechanism for formation of giant polygons on Earth and intermediate-sized polygons on Mars - Results from a prefracture model: Earth and Planetary Science Letters, v. 323324, p. 19-26, https://doi.org/10.1016/j.epsl.2012.01.016.

Feth, J.H., 1951, Structural reconnaissance of the Red Rock Quadrangle, Arizona: U.S. Geological Survey Open-File Report 51-199, 32 p., https://doi.org/10.3133/ofr51199.

Fielding, E.J., Blom, R.G., and Goldstein, R.M., 1998, Rapid subsidence over oil fields measured by SAR interferometry: Geophysical Research Letters, v. 25, no. 17, p. 3215-3218, https://doi.org/10.1029/98GL52260.

Floyd, R.P., 1978, Geodetic bench marks:

Rockville, Md., U.S. Department of Commerce, National Oceanic and Atmospheric Administration, Manual NOS NGS, v. 1, 49 p., available at https://www.ngs.noaa.gov/PUBS_LIB/GeodeticBMs/.

Galloway, D.L., and Burbey, T.J., 2011, ReviewRegional land subsidence accompanying groundwater extraction: Hydrogeology Journal, v. 19, p. 1459-1486, http://doi.org/10.1007/s10040-011-0775-5.

Galloway, D.L., and Hoffmann, J., 2007, The application of satellite differential SAR interferometryderived ground displacements in hydrogeology: Hydrogeology Journal, v. 15, no. 1, p. 133-154, https://doi.org/10.1007/s10040-006-0121-5.

Galloway, D.L., Hudnut, K.W., Ingebritsen, S.E., Phillips, S.P., Peltzer, G., Rogez, F., and Rosen, P.A., 1998, Detection of aquifer system compaction and land subsidence using interferometric synthetic aperture radar, Antelope Valley, Mojave Desert, California: Water Resources Research, v. 34, no. 10, p. 2573-2585. https://doi.org/10.1029/98WR01285.

Galloway, D.L., Jones, D.R., and Ingebritsen, S.E., 1999, Land subsidence in the United States: U.S. Geological Survey Circular 1182, 177 p., https://doi.org/10.3133/cir1182.

Hanson, R.T., 1989, Aquifer-system compaction, Tucson Basin and Avra Valley, Arizona: U.S. Geological Survey Water-Resources Investigations Report 88-4172, 69 p., https://doi.org/10.3133/wri884172.

Harris, R.C., 2004, Giant desiccation cracks in Arizona: Arizona Geological Survey Open-File Report 04-01, 93 p., http://repository.azgs.az.gov/uri_gin/azgs/dlio/1059.

Harris, R.C., and Allison, M.L., 2006, Hazardous cracks running through Arizona: Geotimes, August 2006, p. 24-27.
Helm, D.C.,1978, Field verification of a one-dimensional mathematical model for transient compaction and expansion of a confined aquifer system, verification of mathematical and physical models in hydraulic engineering, in Proceedings of the 26th Hydraulic Division Specialty Conference: College Park, Md., American Society of Civil Engineers, p. 189-196.

Hernandez-Marin, M., and Burbey, T.J., 2010, Controls on initiation and propagation of pumping-induced earth fissures-Insights from numerical simulations: Hydrogeology Journal, v. 18, no. 8, p. 1773-1785, https://doi.org/10.1007/s10040-010-0642-9.

Hernandez-Marin, M., and Burbey T.J., 2012, Fault-controlled deformation and stress from pumping-induced groundwater flow: Journal of Hydrology, v. 428-429, p. 80-93, https://doi.org/10.1016/j.jhydrol.2012.01.025.

Hoffmann, J., Zebker, H.A., Galloway, D.L., and Amelung, F., 2001, Seasonal subsidence and rebound in Las Vegas Valley, Nevada, observed by synthetic aperture radar interferometry: Water Resources Research, v. 37, no. 6, p. 1551-1566, https://doi.org/10.1029/2000WR900404.

Holzer, T.L., 1998, The history of the aquitard-drainage model in land subsidence case studies and current research, in Borchers, J.W., ed., Land subsidence case studies and current research: Proceedings of the Dr. Joseph F. Poland symposium on land subsidence, Association of Engineering Geologists Special Publication no. 8, p. 7-12.

Holzer, T.L., 2000, Methods for prediction of Earth fissures and surface faults caused by groundwater withdrawalThe fragile territory: Congresso Nazionale dei Geologi, 10th, Rome, Italy, 2000, Consiglio Nazionale dei Geologi, p. 179-185.

Holzer, T.L., 2010, Implications of ground-deformation measurements across Earth fissures in subsidence areas in the southwestern USA, in Carreón-Freyre, D., Cerca, M., and Galloway, D.I., eds., Land subsidence, associated hazards and the role of natural resources development: International Association of Hydrological Sciences Publication 339, p. 9-19.

Howle, J.F., Langbein, J.O., Farrar, C.D., and Wilkinson, S.K., 2003, Deformation near the Casa Diablo geothermal well field and related processes Long Valley Caldera, Eastern California, 1993-2000, in Sorey, M.L., McConnell, V.S., and Roeloffs, E., eds., Crustal unrest in Long Valley Caldera, California; new interpretations from geophysical and hydrologic monitoring and deep drilling: Journal of Volcanology and Geothermal Research, v. 127, no. 3-4, p. 365-390, https://doi.org/10.1016/S0377-0273(03)00177-X. 
Huang, H., 2005, Depth of investigation for small broadband electromagnetic sensors: Geophysics, v. 70, no. 6, 135-142, https://doi.org/10.1190/1.2122412.

Ireland, R.L, Poland, J.F., and Riley, F.S., 1984, Land subsidence in the San Joaquin Valley, California, as of 1980: U.S. Geological Survey Professional Paper 437-I, 93 p., https://doi.org/10.3133/pp437I.

Jachens, R.C., and Holzer, T.L., 1982, Differential compaction mechanism for Earth fissures near Casa Grande, Arizona: Geological Society of America Bulletin, v. 93, no. 10, p. 998-1012, https://doi.org/10.1130/00167606(1982)93\%3C998:DCMFEF\%3E2.0.CO;2.

James M. Montgomery and Associates, 1981, Water supply investigation, Fort Irwin, San Bernardino County, California-Phase II, Final report on ground water basin development and management: Consultant's report prepared for and in the files of the U.S. Army Corps of Engineers, Sacramento District, variously paged.

Keller, G.V., and Frischknecht, F.C., 1966, Electrical methods in geophysical prospecting: Oxford, United Kingdom, Pergamon Press, Inc., 884 p.

Kodikara, J., and Choi, X., 2006, A simplified analytical model for desiccation cracking of clay layers in laboratory tests, in Miller, G.A., Zapata, C.E., Houston, S.L., and Fredlund, D.G., eds., Unsaturated Soils 2006 Conference, Carefree, Ariz., April 2, 2006, p. 2558-2569.

Kunkel, F., and Riley, F.S., 1959, Geologic reconnaissance and test-well drilling, Camp Irwin, California: U.S. Geological Survey Water-Supply Paper 1460-F, p. 233-271, https://pubs.usgs.gov/wsp/1460f/report.pdf.

Leake, S.A., and Prudic, D.E., 1991, Documentation of a computer program to simulate aquifer-system compaction using the modular finite-difference ground-water flow model: U.S. Geological Survey Techniques of WaterResources Investigations, book 6, chap. A2, 68 p., https://pubs.usgs.gov/twri/twri6a2/pdf/TWRI_6-A2.pdf.

Loke, M.H., 2004, Tutorial-2-D and 3-D electrical imaging surveys: Penang, Malaysia, Geotomo Software, 128 p., ftp://geom.geometrics.com/pub/mag/G-857_MagCD/ OhmMapper/COURSENOTES.pdf.

Mani, R., Kadau, D., and Herrmann, H.J., 2013, Liquid migration in sheared unsaturated granular media: Granular Matter, v. 15, no. 4, p. 447-454.

Massonnet, D., Rossi, M., Carmona, C., Adragna, F., Peltzer, G., Feigl, K., and Rabaute, T., 1993, The displacement field of the Landers earthquake mapped by radar interferometry: Nature, v. 364, p. 138-142, https://doi.org/10.1038/364138a0.
Massonnet, D., Briole, P., and Arnaud, A., 1995, Deflation of Mount Etna monitored by spaceborne radar interferometry: Nature, v. 375, p. 567-570.

Massonnet, D., Holzer, T., and Vadon, H., 1997, Land subsidence caused by the East Mesa geothermal field, California, observed using SAR interferometry: Geophysical Research Letters, v. 24, no. 8, p. 901-904, https://doi.org/10.1029/97GL00817.

Meinzer, O.E., 1928, Compressibility and elasticity of artesian aquifers: Economic Geology, v. 23, no. 3, p. 263-291, https://doi.org/10.2113/gsecongeo.23.3.263.

Mendenhall, W.C., 1909, Some desert watering places in southeastern California and southwestern Nevada: U.S. Geological Survey Water Supply Paper 224, 98 p., https://doi.org/10.3133/wsp224.

Messina, P., Stoffer, P., and Smith, W.C., 2005, Macropolygon morphology, development, and classification on North Panamint and Eureka playas, Death Valley National Park, CA: Earth- Science Reviews, v. 73, no. 1-4, p. 309-322, https://doi.org/10.1016/j.earscirev.2005.04.011.

Miller, D.M., and Yount, J.C., 2002, Late Cenozoic tectonic evolution of the north-central Mojave Desert inferred from fault history and physiographic evolution of the Fort Irwin area, California, in Glazner, A.F., Walker, J.D., and Bartley, J.M., eds., Geologic evolution of the Mojave Desert and Southwestern Basin and Range: Boulder, Colo., Geological Society of America Memoir, v. 195, p. 173-197.

Miller, D.M., Menges, C.M., and Lidke, D.J., 2014, Generalized surficial geologic map of the Fort Irwin area, San Bernardino County, California, chap. B of Buesch, D.C., ed., Geology and geophysics applied to groundwater hydrology at Fort Irwin, California: U.S. Geological Survey Open-File Report 2013-1024, 11 p., scale 1:100,000, https://doi.org/10.3133/ofr20131024B.

Mitarai, N., and Nori, F., 2006, Wet granular materials: Advances in Physics, v. 55, no.1-2, p. 1-45, https://doi.org/10.1080/00018730600626065.

National Oceanic and Atmospheric Administration, 1984, Standards and specifications for Geodetic control networks: Rockville Md., Federal Geodetic Control Committee, 167 p.

National Oceanic and Atmospheric Administration, 1994, Annual summary, California, 1994: Asheville, N.C., Climatological Data, v. 98, no. 13. accessed 1999 at http://www.ncdc.noaa.gov/IPS/cd/cd.html.

National Oceanic and Atmospheric Administration, 2010, Annual summary, California, 2010: Asheville, N.C., Climatological Data, v. 114, no. 13, accessed September 9, 2015, at http://www.ncdc.noaa.gov/IPS/cd/cd.html. 
National Oceanic and Atmospheric Administration, 2014, Annual summary, California, 2014: Asheville, N.C., Climatological Data, v. 118, no. 13, accessed September 9, 2015, at http://www.ncdc.noaa.gov/IPS/cd/cd.html.

Neal, J.T., Langer, A.M., and Kerr, P.F., 1968, Giant desiccation polygons of Great Basin playas: Geological Society of America Bulletin, v. 79, no. 1, p. 69-90, https://doi.org/10.1130/0016-7606(1968)79[69:GDPOGB] 2.0.CO;2.

Pacheco, J., Arzate, J., Rojas, E., Arroyo M., Yutsis, V., and Ochoa, G., 2006, Delimitation of ground failure zones due to land subsidence using gravity data and finite element modeling in the Queretaro Valley, Mexico: Engineering Geology, v. 84, no. 3-4, p. 143-160, https://doi.org/10.1016/j.enggeo.2005.12.003.

Pacheco-Martínez, J., and Arzate-Flores, J., 2007, Análisis multicapa de la subsidencia en el valle de Querétaro, México: Revista Mexicana de Ciencias Geológicas, v. 24, no. 3, p. 389-402.

Peron, H., Delenne, J.Y., Laloui, L., and El Youssoufi, M.S., 2009, Discrete element modelling of drying shrinkage and cracking of soils: Computers and Geotechnics, v. 36, no. 1-2, p. 61-69, https://doi.org/10.1016/j.compgeo.2008.04.002.

Poland, J.F., ed., 1984, Guidebook to studies of land subsidence due to ground-water withdrawal, v. 40 of UNESCO Studies and Reports in Hydrology: Paris, United Nations Educational, Scientific and Cultural Organization, 305 p., 5 appendices.

Pollard, D.D., and Aydin, A., 1988, Progress in understanding jointing over the past century: Geological Society of America Bulletin, v. 100, no. 8, p. 1181-1204, https://doi.org/10.1130/0016-7606(1988)100<1181:PIUJOT $>2.3 . \mathrm{CO} ; 2$.

Reynolds, J.M., 2011, An introduction to applied and environmental geophysics: Chichester, United Kingdom, John Wiley \& Sons, Ltd., 712 p.

Riley, F.S., 1969, Analysis of borehole extensometer data from central California, in Tison, L.J., ed., Land subsidence: International Association of Hydrological Sciences Publication 89, v. 2, p. 423-431.

Riley, F.S., 1998, Mechanics of aquifer systems-The scientific legacy of Joseph F. Poland, in Borchers, J., ed., Land subsidence - Case studies and current research: Proceedings of the Dr. Joseph F. Poland symposium on land subsidence, Association of Engineering Geologists Special Publication 8, p. 13-27.
Schermer, E.R., Luyendyk, B.P., and Cisowski, S., 1996, Late Cenozoic structure and tectonics of the northern Mojave Desert: Tectonics, v. 15, no. 5, p. 905-932, https://doi.org/10.1029/96TC00131.

Scholtès, L., and Donzè, F.-V., 2012, Modelling progressive failure in fractured rock masses using a 3D discrete element method: International Journal of Rock Mechanics \& Mining Sciences, v. 52, p. 18-30, https://doi.org/10.1016/j.ijrmms.2012.02.009.

Scholtès, L., Nicot, F., and Darve, F., 2009, Discrete Modelling of Capillary Mechanisms in Multi-Phase Granular Media: Computer Modeling in Engineering and Sciences, v. 52, p. 297-318, https://doi.org/10.3970/cmes.2009.052.297.

Shin, H., and Santamarina, J.C., 2011, Desiccation cracks in saturated fine-grained soils-Particle-level phenomena and effective-stress analysis: Géotechnique, v. 61, no. 11, p. 961-972, https://doi.org/10.1680/geot.8.P.012.

Sima, J., Jiang, M., and Zhou, C., 2014, Numerical simulation of desiccation cracking in a thin clay layer using $3 \mathrm{D}$ discrete element modeling: Computers and Geotechnics, v. 56, p. 168-180, https://doi.org/10.1016/j.compgeo.2013.12.003.

Šmilauer, V., Catalano, E., Chareyre, B., Dorofeenko, S., Duriez, J., Gladky, A., Kozicki, J., Modenese, C., Scholtés, L., Sibille, L., Stransky, J., and Thoeni, K., 2015, Yade documentation (2nd ed.): The Yade Project, https://www.yade-dem.org/publi/ documentation_2nd_ed/YadeReference.pdf.

Sneed, M., and Galloway, D.L., 2000, Aquifer-system compaction and land subsidence-Measurements, analyses, and simulations - the Holly site, Edwards Air Force Base, Antelope Valley, California: U.S. Geological Survey Water-Resources Investigations Report 00-4015, 65 p., https://doi.org/10.3133/wri20004015.

Sneed, M., Brandt, J., and Solt, M., 2013, Land subsidence along the Delta-Mendota Canal in the northern part of the San Joaquin Valley, California, 2003-10: U.S. Geological Survey Scientific Investigations Report 2013-5142, 87 p., https://doi.org/10.3133/sir20135142.

Telford, W.M., Geldart, L.P., and Sheriff, R.E., 1990, Applied geophysics (2nd ed.): Cambridge, United Kingdom, Cambridge University Press, $770 \mathrm{p}$.

Terzaghi, K., 1925, Principles of soil mechanics: IVSettlement and consolidation of clay: Erdbaumechanik, v. 95 , no. 3 , p. $874-878$. 
Thayer, D.C., Ball, L.B., Densmore, J.N., Swarzenski, P., and Johnson, C., 2018, Electrical Resistivity Tomography Data at Fort Irwin National Training Center, San Bernardino County, California, 2015 and 2017: U.S. Geological Survey, https://doi.org/10.5066/F77W6BF0.

Wilson F. So and Associates, Inc., 1989, U.S. Army National Training Center at Fort Irwin, California, water basin development plan, final report: Consultant's report prepared for and in the files of the Commander of the National Training Center at Fort Irwin, 85 p.

Won, I.J., Keiswetter, D., Fields, G., and Sutton, L., 1996, GEM-2 - A new multifrequency electromagnetic sensor: Journal of Environmental and Engineering Geophysics, v. 1, no. 2, p. 129-137, https://doi.org/10.4133/JEEG1.2.129.
Yamashita, K.M., and Kaiser, W.D., 1992, Using first-order class II geodetic leveling procedures to monitor vertical displacement in Ewert, J.W., and Swanson, D.A., eds., Monitoring volcanoes: Techniques and strategies used by the staff of the Cascade Volcano Observatory, 1980-90: U.S. Geological Survey Bulletin 1966, p. 1-7.

Yamashita, K.M., and Yamashita, J.M., 1992, Using the MC-II handheld computer while running first or second order leveling surveys: U.S. Geological Survey Open-File Report 92-184, 17 p., https://doi.org/10.3133/ofr92184.

Yount, J.C., Schermer, E.R., Felger, T.J., Miller, D.M., and Stephens, K.A., 1994, Preliminary geologic map of Fort Irwin Basin, north-central Mojave Desert, California: U.S. Geological Survey Open-File Report 94-173, 25 p., https://doi.org/10.3133/ofr94173.

Zebker, H.A., Rosen, P.A., and Hensley, S., 1997, Atmospheric effects in interferometric synthetic aperture radar surface deformation and topographic maps: Journal of Geophysical Research, v. 102, p. 7547-7563, https://doi.org/10.1029/96JB03804. 


\section{Appendix 1. Summary of Construction Data for Production and Monitoring Wells in the Bicycle Basin, Fort Irwin National Training Center, California}

[Location of wells are shown in figure 2. Abbreviations: Bc, basement complex; D, well destroyed; ft, feet; ID, identification; M, monitoring well; m, meters; NA, well not in model domain; NAVD 88, North American Vertical Datum of 1988; P, production well; Qoa, Quaternary older alluvium; QToa, QToa1, QToa2, Quaternary-Tertiary older alluvium; QTol, Quaternary-Tertiary older lacustrine; Qya, Quaternary younger alluvium; Tog, Tertiary older gravels; Tyg, Tertiary younger gravels; U, unused well; - , data not available]

\begin{tabular}{|c|c|c|c|c|c|c|c|c|c|c|c|c|c|c|c|}
\hline \multirow{2}{*}{$\begin{array}{l}\text { State well } \\
\text { number }\end{array}$} & \multirow{2}{*}{$\begin{array}{l}\text { Short } \\
\text { ID }\end{array}$} & \multirow[t]{2}{*}{ Local ID } & \multirow{2}{*}{$\begin{array}{l}\text { Well } \\
\text { type }\end{array}$} & \multirow[t]{2}{*}{ Date drilled } & \multirow[t]{2}{*}{ Geology } & \multicolumn{2}{|c|}{$\begin{array}{c}\text { Land-surface altitude, } \\
\text { NAVD } 88\end{array}$} & \multicolumn{2}{|c|}{ Hole depth } & \multicolumn{2}{|c|}{ Well depth } & \multicolumn{2}{|c|}{ Screen top } & \multicolumn{2}{|c|}{ Screen bottom } \\
\hline & & & & & & (ft) & (m) & (ft) & (m) & (ft) & (m) & (ft) & (m) & (ft) & (m) \\
\hline $14 \mathrm{~N} / 3 \mathrm{E}-13 \mathrm{~K} 1 \mathrm{~S}$ & $-13 \mathrm{~K} 1$ & B-1 & $\mathrm{P}^{1}$ & January 1955 & QToa, Tyg & 2,398 & 731 & 600 & 183 & 600 & 183 & 180 & 55 & 580 & 125 \\
\hline $14 \mathrm{~N} / 3 \mathrm{E}-13 \mathrm{M} 1 \mathrm{~S}$ & $-13 \mathrm{M} 1$ & B-4 & $\mathrm{P}$ & May 1965 & QToa, Tyg & 2,419 & 737 & 614 & 187 & 600 & - & 226 & 69 & 594 & 181 \\
\hline $14 \mathrm{~N} / 3 \mathrm{E}-13 \mathrm{M} 2 \mathrm{~S}$ & $-13 \mathrm{M} 2$ & BLA2-1 & M & June 1997 & Tyg & 2,418 & 737 & 600 & 183 & 600 & 183 & 580 & 177 & 600 & 183 \\
\hline $14 \mathrm{~N} / 3 \mathrm{E}-13 \mathrm{M} 3 \mathrm{~S}$ & $-13 \mathrm{M} 3$ & BLA2-2 & M & June 1997 & QToa2, 1 & 2,418 & 737 & 600 & 183 & 440 & 134 & 420 & 128 & 440 & 134 \\
\hline $14 N / 3 E-13 M 4 S$ & $-13 \mathrm{M} 4$ & BLA2-3 & M & June 1997 & QToa2 & 2,418 & 737 & 600 & 183 & 330 & 101 & 310 & 94 & 330 & 101 \\
\hline $14 \mathrm{~N} / 3 \mathrm{E}-14 \mathrm{H} 1 \mathrm{~S}$ & $-14 \mathrm{H} 1$ & B-2 & $\mathrm{P}^{1}$ & November 1964 & QToa & 2,423 & 738 & 602 & 183 & 602 & 183 & 230 & 70 & 585 & 178 \\
\hline 14N/3E-14P1S & $-14 \mathrm{P} 1$ & B-6 & $\mathrm{P}$ & January 1988 & QToa & 2,380 & 725 & 540 & 165 & 535 & 163 & 380 & 116 & 525 & 160 \\
\hline $14 \mathrm{~N} / 3 \mathrm{E}-14 \mathrm{P} 2 \mathrm{~S}$ & $-14 \mathrm{P} 2$ & TH-7 & M & January 1988 & QToa & 2,380 & 725 & 800 & 244 & 535 & 163 & 380 & 116 & 525 & 160 \\
\hline $14 \mathrm{~N} / 3 \mathrm{E}-22 \mathrm{~N} 1 \mathrm{~S}$ & $-22 \mathrm{~N} 1$ & BA1-1 & $\mathrm{M}^{1}$ & March 1993 & $\mathrm{Bc}$ & 2,418 & 737 & - & - & 260 & 79 & 240 & 73 & 260 & 79 \\
\hline $14 N / 3 E-22 N 2 S$ & $-22 \mathrm{~N} 2$ & BA1-2 & $\mathrm{M}^{1}$ & March 1993 & QToa & 2,418 & 737 & - & - & 170 & 52 & 150 & 46 & 170 & 52 \\
\hline $14 \mathrm{~N} / 3 \mathrm{E}-22 \mathrm{P} 1 \mathrm{~S}$ & $-22 \mathrm{P} 1$ & B-3 & $\mathrm{D}$ & - & QToa & 2,432 & 741 & 532 & 162 & 478 & 146 & - & - & - & - \\
\hline $14 \mathrm{~N} / 3 \mathrm{E}-23 \mathrm{~B} 1 \mathrm{~S}$ & $-23 \mathrm{~B} 1$ & BLA4-1 & M & December 2007 & Tyg & 2,377 & 725 & 865 & 264 & 850 & 259 & 710 & 216 & 730 & 223 \\
\hline $14 \mathrm{~N} / 3 \mathrm{E}-23 \mathrm{~B} 2 \mathrm{~S}$ & $-23 \mathrm{~B} 2$ & BLA4-2 & M & December 2007 & QToa1 & 2,377 & 725 & 865 & 264 & 460 & 140 & 440 & 134 & 460 & 140 \\
\hline $14 \mathrm{~N} / 3 \mathrm{E}-23 \mathrm{~B} 3 \mathrm{~S}$ & $-23 \mathrm{~B} 3$ & BLA4-3 & M & December 2007 & QToa2 & 2,377 & 725 & 865 & 264 & 280 & 85 & 260 & 79 & 280 & 85 \\
\hline $14 \mathrm{~N} / 3 \mathrm{E}-23 \mathrm{G} 1 \mathrm{~S}$ & $-23 \mathrm{G} 1$ & BX-2 & M & October 1980 & QToa, QTol, Tyg & 2,361 & 720 & 748 & 228 & 747 & 228 & 178 & 54 & 737 & 225 \\
\hline $14 \mathrm{~N} / 3 \mathrm{E}-24 \mathrm{H} 1 \mathrm{~S}$ & $-24 \mathrm{H} 1$ & BX-1 & M & October 1980 & Qoa, QToa & 2,362 & 720 & 414 & 126 & 413 & 126 & 183 & 56 & 403 & 123 \\
\hline 14N/3E-24Q1S & $-24 Q 1$ & BLA3-1 & M & July 1997 & Tog & 2,356 & 718 & 900 & 274 & 898 & 274 & 878 & 268 & 898 & 274 \\
\hline $14 N / 3 E-24 Q 2 S$ & $-24 Q 2$ & BLA3-2 & M & July 1997 & Tog & 2,356 & 718 & 900 & 274 & 745 & 227 & 725 & 221 & 745 & 227 \\
\hline $14 \mathrm{~N} / 3 \mathrm{E}-24 \mathrm{Q} 3 \mathrm{~S}$ & $-24 Q 3$ & BLA3-3 & M & July 1997 & Tyg & 2,356 & 718 & 900 & 274 & 610 & 186 & 590 & 180 & 610 & 186 \\
\hline $14 \mathrm{~N} / 3 \mathrm{E}-24 \mathrm{Q} 4 \mathrm{~S}$ & $-24 Q 4$ & BLA3-4 & M & July 1997 & QToa1 & 2,356 & 718 & 900 & 274 & 450 & 137 & 430 & 131 & 450 & 137 \\
\hline $14 N / 3 E-24 Q 5 S$ & $-24 Q 5$ & BLA3-5 & M & July 1997 & QToa2 & 2,356 & 718 & 900 & 274 & 310 & 94 & 290 & 88 & 310 & 94 \\
\hline $14 \mathrm{~N} / 3 \mathrm{E}-26 \mathrm{~K} 1 \mathrm{~S}$ & $-26 \mathrm{~K} 1$ & BLA5-1 & M & March 2011 & Tyg & 2,345 & 715 & 370 & 113 & 360 & 110 & 320 & 98 & 340 & 104 \\
\hline
\end{tabular}


Appendix 1. Summary of construction data for production and monitoring wells in the Bicycle Basin, Fort Irwin Training Center, California.-Continued

[Location of wells are shown in figure 2. Abbreviations: Bc, basement complex; D, well destroyed; ft, feet; ID, identification; M, monitoring well; m, meters; NA, well not in model domain; NAVD 88, North American Vertical Datum of 1988; P, production well; Qoa, Quaternary older alluvium; QToa, QToa1, QToa2, Quaternary-Tertiary older alluvium; QTol, Quaternary-Tertiary older lacustrine; Qya, Quaternary younger alluvium; Tog, Tertiary older gravels; Tyg, Tertiary younger gravels; U, unused well; - - data not available]

\begin{tabular}{|c|c|c|c|c|c|c|c|c|c|c|c|c|c|c|c|}
\hline \multirow{2}{*}{$\begin{array}{l}\text { State well } \\
\text { number }\end{array}$} & \multirow{2}{*}{$\begin{array}{l}\text { Short } \\
\text { ID }\end{array}$} & \multirow[t]{2}{*}{ Local ID } & \multirow{2}{*}{$\begin{array}{l}\text { Well } \\
\text { type }\end{array}$} & \multirow[t]{2}{*}{ Date drilled } & \multirow[t]{2}{*}{ Geology } & \multicolumn{2}{|c|}{$\begin{array}{c}\text { Land-surface altitude, } \\
\text { NAVD } 88\end{array}$} & \multicolumn{2}{|c|}{ Hole depth } & \multicolumn{2}{|c|}{ Well depth } & \multicolumn{2}{|c|}{ Screen top } & \multicolumn{2}{|c|}{ Screen bottom } \\
\hline & & & & & & (ft) & (m) & $(\mathrm{ft})$ & (m) & (ft) & (m) & (ft) & (m) & (ft) & (m) \\
\hline $14 \mathrm{~N} / 3 \mathrm{E}-26 \mathrm{~K} 2 \mathrm{~S}^{2}$ & $-26 \mathrm{~K} 2$ & BLA5-2 & $\mathrm{M}$ & March 2011 & QToa & 2,345 & 715 & 370 & 113 & 210 & 64 & 190 & 58 & 210 & 64 \\
\hline $14 N / 3 E-26 K 3 S$ & $-26 \mathrm{~K} 3$ & BLA5-3 & M & March 2011 & QToa & 2,345 & 715 & 370 & 113 & 210 & 64 & 190 & 58 & 210 & 64 \\
\hline $14 \mathrm{~N} / 3 \mathrm{E}-26 \mathrm{~K} 4 \mathrm{~S}$ & $-26 \mathrm{~K} 4$ & BLA5B 1 & M & March 2011 & QToa & 2,345 & 715 & 280 & 85 & 270 & 82 & 250 & 76 & 270 & 82 \\
\hline $14 \mathrm{~N} / 3 \mathrm{E}-27 \mathrm{E} 1 \mathrm{~S}$ & $-27 \mathrm{E} 1$ & BP-2, MW-12 & M & - & Tog & 2,401 & 732 & - & - & - & - & - & - & - & - \\
\hline $14 N / 3 E-27 E 2 S$ & $-27 \mathrm{E} 2$ & BP-3, MW-13 & M & - & Tog & 2,401 & 732 & - & - & - & - & - & - & - & - \\
\hline $14 N / 3 E-27 E 3 S$ & $-27 \mathrm{E} 3$ & BP-4, MW-14 & M & - & Tog & 2,401 & 732 & - & - & - & - & - & - & - & - \\
\hline $14 \mathrm{~N} / 3 \mathrm{E}-28 \mathrm{~A} 1 \mathrm{~S}$ & $-28 \mathrm{~A} 1$ & BP-MW22 & M & - & Tog & 2,411 & 735 & - & - & 151.28 & 46 & - & - & - & - \\
\hline $14 \mathrm{~N} / 3 \mathrm{E}-28 \mathrm{H} 1 \mathrm{~S}$ & $-28 \mathrm{H} 1$ & BP-1, MW-11 & M & - & Tog & 2,407 & 734 & - & - & - & - & - & - & - & - \\
\hline $14 \mathrm{~N} / 3 \mathrm{E}-28 \mathrm{H} 2 \mathrm{~S}$ & $-28 \mathrm{H} 2$ & BP-MW21 & M & - & Tog & 2,401 & 732 & - & - & 164.32 & 50 & - & - & - & - \\
\hline $14 \mathrm{~N} / 3 \mathrm{E}-35 \mathrm{C} 1 \mathrm{~S}$ & $-35 \mathrm{C} 1$ & B-9_Aprt & $\mathrm{U}$ & March 1963 & $\mathrm{Bc}$ & 2,352 & 717 & 250 & 76 & 245 & - & 125 & 38 & 245 & 75 \\
\hline $14 \mathrm{~N} / 3 \mathrm{E}-35 \mathrm{C} 2 \mathrm{~S}$ & $-35 \mathrm{C} 2$ & BLA1-1 & M & May 1994 & $\mathrm{Bc}$ & 2,357 & 719 & 200 & 61 & 175 & 53 & 155 & 47 & 175 & 53 \\
\hline $14 \mathrm{~N} / 3 \mathrm{E}-35 \mathrm{C} 3 \mathrm{~S}$ & $-35 \mathrm{C} 3$ & BLA1-2 & M & May 1994 & Qya & 2,357 & 719 & 200 & 61 & 25 & 8 & 15 & 5 & 25 & 8 \\
\hline $14 \mathrm{~N} / 3 \mathrm{E}-35 \mathrm{C} 4 \mathrm{~S}$ & $-35 \mathrm{C} 4$ & W3 & M & August 1997 & $\mathrm{Bc}$ & 2,350 & 716 & 300 & 91 & 300 & 91 & 200 & 61 & 300 & 91 \\
\hline $14 \mathrm{~N} / 4 \mathrm{E}-18 \mathrm{~N} 1 \mathrm{~S}$ & $-18 \mathrm{~N} 1$ & B-5 & $\mathrm{P}$ & March 1983 & QToa, Tyg, Tog & 2,378 & 725 & 803 & 245 & 800 & 244 & 300 & 91 & 780 & 238 \\
\hline $14 \mathrm{~N} / 4 \mathrm{E}-18 \mathrm{~N} 2 \mathrm{~S}$ & $-18 \mathrm{~N} 2$ & B-5A & M & February 1983 & QToa & 2,380 & 725 & 803 & 245 & 803 & 245 & 302 & 92 & 305 & 93 \\
\hline
\end{tabular}

${ }^{1}$ The well was destroyed.

${ }^{2}$ The well was sealed and abandoned; it was replaced by site BLA5B. 


\section{Appendix 2. Water-Level Data for Selected Wells in Bicycle Basin, Fort Irwin National Training Center, California, 1955-2017}

[State well No.: See Well-Numbering System in text. Abbreviations: ID, identification number; mm/dd/yyyy, month/day/year; NAVD 88, North American Vertical Datum of 1988, , well is dry]

\begin{tabular}{|c|c|c|c|c|c|c|c|c|}
\hline \multirow[t]{2}{*}{ Site ID } & \multirow{2}{*}{$\begin{array}{c}\text { State } \\
\text { well number }\end{array}$} & \multirow{2}{*}{$\begin{array}{c}\text { Date } \\
\text { (mm/dd/yyyy) }\end{array}$} & \multicolumn{2}{|c|}{$\begin{array}{c}\text { Water-surface altitude, } \\
\text { above NAVD } 88\end{array}$} & \multicolumn{2}{|c|}{$\begin{array}{l}\text { Depth to water, below } \\
\text { land surface }\end{array}$} & \multicolumn{2}{|c|}{$\begin{array}{l}\text { Land-surface altitude, } \\
\text { above NAVD } 88^{1}\end{array}$} \\
\hline & & & (feet) & (meters) & (feet) & (meters) & (feet) & (meters) \\
\hline \multicolumn{9}{|c|}{ Local well ID: B-1 } \\
\hline 351830116364501 & 14N/3E-13K1S & $02 / 01 / 1955$ & $2,226.8$ & 678.80 & 170.9 & 52.10 & $2,397.7$ & 730.90 \\
\hline 351830116364501 & $14 \mathrm{~N} / 3 \mathrm{E}-13 \mathrm{~K} 1 \mathrm{~S}$ & 05/01/1963 & $2,222.7$ & 677.55 & 175 & 53.35 & $2,397.7$ & 730.90 \\
\hline 351830116364501 & $14 \mathrm{~N} / 3 \mathrm{E}-13 \mathrm{~K} 1 \mathrm{~S}$ & 06/01/1967 & $2,218.7$ & 676.33 & 179 & 54.57 & $2,397.7$ & 730.90 \\
\hline 351830116364501 & $14 \mathrm{~N} / 3 \mathrm{E}-13 \mathrm{~K} 1 \mathrm{~S}$ & 01/01/1968 & $2,221.7$ & 677.25 & 176 & 53.65 & $2,397.7$ & 730.90 \\
\hline 351830116364501 & $14 \mathrm{~N} / 3 \mathrm{E}-13 \mathrm{~K} 1 \mathrm{~S}$ & 01/01/1969 & $2,216.7$ & 675.72 & 181 & 55.17 & $2,397.7$ & 730.90 \\
\hline 351830116364501 & $14 \mathrm{~N} / 3 \mathrm{E}-13 \mathrm{~K} 1 \mathrm{~S}$ & 02/01/1970 & $2,216.7$ & 675.72 & 181 & 55.17 & $2,397.7$ & 730.90 \\
\hline 351830116364501 & $14 \mathrm{~N} / 3 \mathrm{E}-13 \mathrm{~K} 1 \mathrm{~S}$ & $12 / 01 / 1978$ & $2,212.7$ & 674.50 & 185 & 56.39 & $2,397.7$ & 730.90 \\
\hline 351830116364501 & $14 \mathrm{~N} / 3 \mathrm{E}-13 \mathrm{~K} 1 \mathrm{~S}$ & 04/10/1979 & $2,211.3$ & 674.08 & 186.39 & 56.82 & $2,397.7$ & 730.90 \\
\hline 351830116364501 & $14 \mathrm{~N} / 3 \mathrm{E}-13 \mathrm{~K} 1 \mathrm{~S}$ & 07/29/1980 & $2,209.9$ & 673.65 & 187.83 & 57.26 & $2,397.7$ & 730.90 \\
\hline 351830116364501 & $14 \mathrm{~N} / 3 \mathrm{E}-13 \mathrm{~K} 1 \mathrm{~S}$ & 01/20/1981 & $2,210.4$ & 673.80 & 187.25 & 57.08 & $2,397.7$ & 730.90 \\
\hline 351830116364501 & $14 \mathrm{~N} / 3 \mathrm{E}-13 \mathrm{~K} 1 \mathrm{~S}$ & 06/24/1982 & $2,200.8$ & 670.88 & 196.89 & 60.02 & $2,397.7$ & 730.90 \\
\hline 351830116364501 & $14 \mathrm{~N} / 3 \mathrm{E}-13 \mathrm{~K} 1 \mathrm{~S}$ & $11 / 16 / 1982$ & $2,204.9$ & 672.13 & 192.83 & 58.78 & $2,397.7$ & 730.90 \\
\hline 351830116364501 & $14 \mathrm{~N} / 3 \mathrm{E}-13 \mathrm{~K} 1 \mathrm{~S}$ & $11 / 15 / 1983$ & $2,203.6$ & 671.73 & 194.14 & 59.18 & $2,397.7$ & 730.90 \\
\hline 351830116364501 & $14 \mathrm{~N} / 3 \mathrm{E}-13 \mathrm{~K} 1 \mathrm{~S}$ & 01/26/1993 & 2,188 & 666.98 & 209.69 & 63.92 & $2,397.7$ & 730.90 \\
\hline 351830116364501 & $14 \mathrm{~N} / 3 \mathrm{E}-13 \mathrm{~K} 1 \mathrm{~S}$ & 09/23/1993 & - & - & - & - & $2,397.7$ & 730.90 \\
\hline 351830116364501 & $14 \mathrm{~N} / 3 \mathrm{E}-13 \mathrm{~K} 1 \mathrm{~S}$ & 03/02/2005 & $2,131.6$ & 649.78 & 266.12 & 81.12 & $2,397.7$ & 730.90 \\
\hline 351830116364501 & $14 \mathrm{~N} / 3 \mathrm{E}-13 \mathrm{~K} 1 \mathrm{~S}$ & $11 / 06 / 2007$ & - & - & - & - & $2,397.7$ & 730.90 \\
\hline 351830116364501 & $14 \mathrm{~N} / 3 \mathrm{E}-13 \mathrm{~K} 1 \mathrm{~S}$ & $12 / 19 / 2007$ & - & - & - & - & $2,397.7$ & 730.90 \\
\hline 351830116364501 & $14 \mathrm{~N} / 3 \mathrm{E}-13 \mathrm{~K} 1 \mathrm{~S}$ & $02 / 14 / 2008$ & - & - & - & - & $2,397.7$ & 730.90 \\
\hline 351830116364501 & $14 \mathrm{~N} / 3 \mathrm{E}-13 \mathrm{~K} 1 \mathrm{~S}$ & $12 / 13 / 2010$ & - & - & - & - & $2,397.7$ & 730.90 \\
\hline \multicolumn{9}{|c|}{ Local well ID: B-4 } \\
\hline 351829116371201 & $14 \mathrm{~N} / 3 \mathrm{E}-13 \mathrm{M} 1 \mathrm{~S}$ & $06 / 30 / 1965$ & $2,215.8$ & 675.45 & 203 & 61.88 & $2,418.8$ & 737.33 \\
\hline 351829116371201 & $14 \mathrm{~N} / 3 \mathrm{E}-13 \mathrm{M} 1 \mathrm{~S}$ & 04/01/1967 & $2,196.8$ & 669.66 & 222 & 67.67 & $2,418.8$ & 737.33 \\
\hline 351829116371201 & 14N/3E-13M1S & 01/01/1968 & $2,194.8$ & 669.05 & 224 & 68.28 & $2,418.8$ & 737.33 \\
\hline
\end{tabular}


Appendix 2. Water-level data for selected wells in Bicycle Basin, Fort Irwin National Training Center, California, 19552017.-Continued

[State well No.: See Well-Numbering System in text. Abbreviations: ID, identification number; mm/dd/yyyy, month/day/year; NAVD 88, North American Vertical Datum of 1988, -, well is dry]

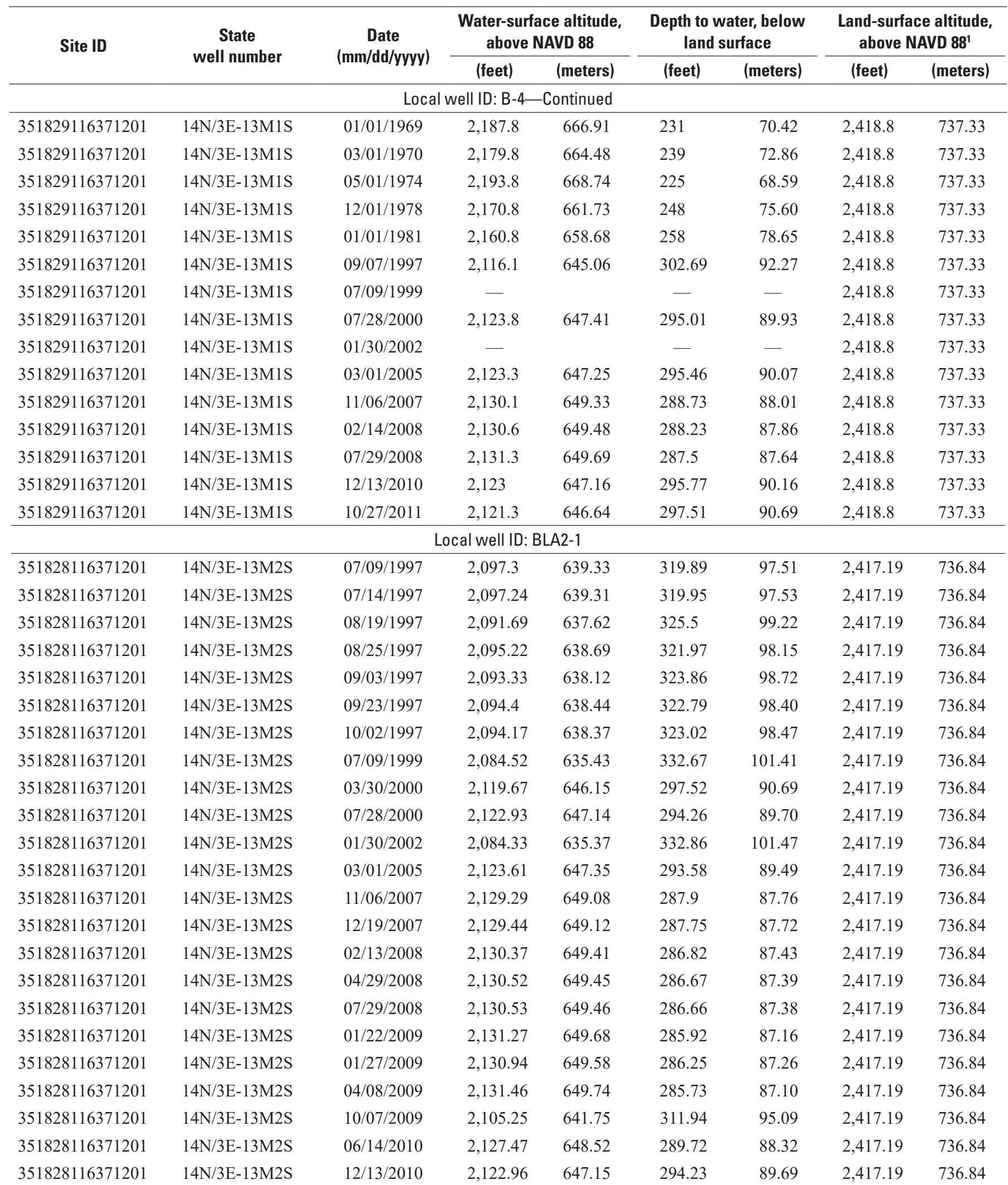


Appendix 2. Water-level data for selected wells in Bicycle Basin, Fort Irwin National Training Center, California, 19552017.-Continued

[State well No.: See Well-Numbering System in text. Abbreviations: ID, identification number; mm/dd/yyyy, month/day/year; NAVD 88, North American Vertical Datum of 1988, - , well is dry]

\begin{tabular}{|c|c|c|c|c|c|c|c|c|}
\hline \multirow[t]{2}{*}{ Site ID } & \multirow{2}{*}{$\begin{array}{c}\text { State } \\
\text { well number }\end{array}$} & \multirow{2}{*}{$\begin{array}{c}\text { Date } \\
\text { (mm/dd/yyyy) }\end{array}$} & \multicolumn{2}{|c|}{$\begin{array}{c}\text { Water-surface altitude, } \\
\text { above NAVD } 88\end{array}$} & \multicolumn{2}{|c|}{$\begin{array}{c}\text { Depth to water, below } \\
\text { land surface }\end{array}$} & \multicolumn{2}{|c|}{$\begin{array}{c}\text { Land-surface altitude, } \\
\text { above NAVD } 88^{1}\end{array}$} \\
\hline & & & (feet) & (meters) & (feet) & (meters) & (feet) & (meters) \\
\hline 351828116371201 & $14 \mathrm{~N} / 3 \mathrm{E}-13 \mathrm{M} 2 \mathrm{~S}$ & $04 / 28 / 2011$ & $2,122.14$ & 646.90 & 295.05 & 89.94 & $2,417.19$ & 736.84 \\
\hline 351828116371201 & $14 \mathrm{~N} / 3 \mathrm{E}-13 \mathrm{M} 2 \mathrm{~S}$ & $10 / 24 / 2011$ & $2,121.64$ & 646.75 & 295.55 & 90.09 & $2,417.19$ & 736.84 \\
\hline 351828116371201 & $14 \mathrm{~N} / 3 \mathrm{E}-13 \mathrm{M} 2 \mathrm{~S}$ & $02 / 21 / 2012$ & $2,120.47$ & 646.39 & 296.72 & 90.45 & $2,417.19$ & 736.84 \\
\hline 351828116371201 & $14 \mathrm{~N} / 3 \mathrm{E}-13 \mathrm{M} 2 \mathrm{~S}$ & $08 / 30 / 2012$ & $2,113.35$ & 644.22 & 303.84 & 92.62 & $2,417.19$ & 736.84 \\
\hline 351828116371201 & $14 \mathrm{~N} / 3 \mathrm{E}-13 \mathrm{M} 2 \mathrm{~S}$ & $04 / 17 / 2013$ & $2,117.4$ & 645.45 & 299.79 & 91.39 & $2,417.19$ & 736.84 \\
\hline 351828116371201 & $14 \mathrm{~N} / 3 \mathrm{E}-13 \mathrm{M} 2 \mathrm{~S}$ & 09/10/2013 & $2,102.64$ & 640.95 & 314.55 & 95.89 & $2,417.19$ & 736.84 \\
\hline 351828116371201 & $14 \mathrm{~N} / 3 \mathrm{E}-13 \mathrm{M} 2 \mathrm{~S}$ & $05 / 20 / 2015$ & $2,117.55$ & 645.50 & 299.64 & 91.34 & $2,417.19$ & 736.84 \\
\hline 351828116371201 & $14 \mathrm{~N} / 3 \mathrm{E}-13 \mathrm{M} 2 \mathrm{~S}$ & $12 / 22 / 2015$ & $2,120.09$ & 646.27 & 297.1 & 90.57 & $2,417.19$ & 736.84 \\
\hline 351828116371201 & $14 \mathrm{~N} / 3 \mathrm{E}-13 \mathrm{M} 2 \mathrm{~S}$ & $05 / 24 / 2016$ & $2,110.01$ & 643.20 & 307.18 & 93.64 & $2,417.19$ & 736.84 \\
\hline 351828116371201 & $14 \mathrm{~N} / 3 \mathrm{E}-13 \mathrm{M} 2 \mathrm{~S}$ & $06 / 06 / 2016$ & $2,104.75$ & 641.60 & 312.44 & 95.24 & $2,417.19$ & 736.84 \\
\hline 351828116371201 & $14 \mathrm{~N} / 3 \mathrm{E}-13 \mathrm{M} 2 \mathrm{~S}$ & $12 / 08 / 2016$ & $2,115.47$ & 644.87 & 301.72 & 91.97 & $2,417.19$ & 736.84 \\
\hline 351828116371201 & $14 \mathrm{~N} / 3 \mathrm{E}-13 \mathrm{M} 2 \mathrm{~S}$ & $03 / 07 / 2017$ & $2,117.21$ & 645.40 & 299.98 & 91.44 & $2,417.19$ & 736.84 \\
\hline 351828116371201 & $14 \mathrm{~N} / 3 \mathrm{E}-13 \mathrm{M} 2 \mathrm{~S}$ & 05/16/2017 & $2,118.29$ & 645.73 & 298.9 & 91.11 & $2,417.19$ & 736.84 \\
\hline \multicolumn{9}{|c|}{ Local well ID: BLA2-2 } \\
\hline 351828116371202 & $14 \mathrm{~N} / 3 \mathrm{E}-13 \mathrm{M} 3 \mathrm{~S}$ & 07/09/1997 & $2,119.42$ & 646.07 & 297.77 & 90.77 & $2,417.19$ & 736.84 \\
\hline 351828116371202 & $14 \mathrm{~N} / 3 \mathrm{E}-13 \mathrm{M} 3 \mathrm{~S}$ & 07/09/1999 & $2,106.41$ & 642.10 & 310.78 & 94.74 & $2,417.19$ & 736.84 \\
\hline 351828116371202 & $14 \mathrm{~N} / 3 \mathrm{E}-13 \mathrm{M} 3 \mathrm{~S}$ & $03 / 30 / 2000$ & $2,119.31$ & 646.04 & 297.88 & 90.80 & $2,417.19$ & 736.84 \\
\hline 351828116371202 & $14 \mathrm{~N} / 3 \mathrm{E}-13 \mathrm{M} 3 \mathrm{~S}$ & $07 / 28 / 2000$ & $2,123.66$ & 647.36 & 293.53 & 89.48 & $2,417.19$ & 736.84 \\
\hline 351828116371202 & $14 \mathrm{~N} / 3 \mathrm{E}-13 \mathrm{M} 3 \mathrm{~S}$ & $01 / 30 / 2002$ & $2,103.12$ & 641.10 & 314.07 & 95.74 & $2,417.19$ & 736.84 \\
\hline 351828116371202 & $14 \mathrm{~N} / 3 \mathrm{E}-13 \mathrm{M} 3 \mathrm{~S}$ & $03 / 01 / 2005$ & $2,123.48$ & 647.31 & 293.71 & 89.53 & $2,417.19$ & 736.84 \\
\hline 351828116371202 & $14 \mathrm{~N} / 3 \mathrm{E}-13 \mathrm{M} 3 \mathrm{~S}$ & $11 / 06 / 2007$ & $2,129.29$ & 649.08 & 287.9 & 87.76 & $2,417.19$ & 736.84 \\
\hline 351828116371202 & $14 \mathrm{~N} / 3 \mathrm{E}-13 \mathrm{M} 3 \mathrm{~S}$ & $12 / 19 / 2007$ & $2,129.43$ & 649.12 & 287.76 & 87.72 & $2,417.19$ & 736.84 \\
\hline 351828116371202 & $14 \mathrm{~N} / 3 \mathrm{E}-13 \mathrm{M} 3 \mathrm{~S}$ & $02 / 13 / 2008$ & $2,130.33$ & 649.40 & 286.86 & 87.44 & $2,417.19$ & 736.84 \\
\hline 351828116371202 & $14 \mathrm{~N} / 3 \mathrm{E}-13 \mathrm{M} 3 \mathrm{~S}$ & $04 / 29 / 2008$ & $2,130.5$ & 649.45 & 286.69 & 87.39 & $2,417.19$ & 736.84 \\
\hline 351828116371202 & $14 \mathrm{~N} / 3 \mathrm{E}-13 \mathrm{M} 3 \mathrm{~S}$ & $07 / 29 / 2008$ & $2,130.52$ & 649.45 & 286.67 & 87.39 & $2,417.19$ & 736.84 \\
\hline 351828116371202 & $14 \mathrm{~N} / 3 \mathrm{E}-13 \mathrm{M} 3 \mathrm{~S}$ & $01 / 22 / 2009$ & $2,131.27$ & 649.68 & 285.92 & 87.16 & $2,417.19$ & 736.84 \\
\hline 351828116371202 & $14 \mathrm{~N} / 3 \mathrm{E}-13 \mathrm{M} 3 \mathrm{~S}$ & $01 / 27 / 2009$ & $2,130.89$ & 649.57 & 286.3 & 87.27 & $2,417.19$ & 736.84 \\
\hline 351828116371202 & $14 \mathrm{~N} / 3 \mathrm{E}-13 \mathrm{M} 3 \mathrm{~S}$ & $04 / 08 / 2009$ & $2,131.44$ & 649.73 & 285.75 & 87.11 & $2,417.19$ & 736.84 \\
\hline 351828116371202 & $14 \mathrm{~N} / 3 \mathrm{E}-13 \mathrm{M} 3 \mathrm{~S}$ & $10 / 07 / 2009$ & $2,124.06$ & 647.48 & 293.13 & 89.36 & $2,417.19$ & 736.84 \\
\hline
\end{tabular}


Appendix 2. Water-level data for selected wells in Bicycle Basin, Fort Irwin National Training Center, California, 19552017.-Continued

[State well No.: See Well-Numbering System in text. Abbreviations: ID, identification number; mm/dd/yyyy, month/day/year; NAVD 88, North American Vertical Datum of $1988, \ldots$, well is dry]

\begin{tabular}{|c|c|c|c|c|c|c|c|c|}
\hline \multirow[t]{2}{*}{ Site ID } & \multirow{2}{*}{$\begin{array}{c}\text { State } \\
\text { well number }\end{array}$} & \multirow{2}{*}{$\begin{array}{c}\text { Date } \\
\text { (mm/dd/yyyy) }\end{array}$} & \multicolumn{2}{|c|}{$\begin{array}{c}\text { Water-surface altitude, } \\
\text { above NAVD } 88\end{array}$} & \multicolumn{2}{|c|}{$\begin{array}{l}\text { Depth to water, below } \\
\text { land surface }\end{array}$} & \multicolumn{2}{|c|}{$\begin{array}{c}\text { Land-surface altitude, } \\
\text { above NAVD } 88^{1}\end{array}$} \\
\hline & & & (feet) & (meters) & (feet) & (meters) & (feet) & (meters) \\
\hline \multicolumn{9}{|c|}{ Local well ID: BLA2-2-Continued } \\
\hline 351828116371202 & $14 \mathrm{~N} / 3 \mathrm{E}-13 \mathrm{M} 3 \mathrm{~S}$ & $06 / 14 / 2010$ & $2,127.57$ & 648.55 & 289.62 & 88.29 & $2,417.19$ & 736.84 \\
\hline 351828116371202 & $14 \mathrm{~N} / 3 \mathrm{E}-13 \mathrm{M} 3 \mathrm{~S}$ & $12 / 13 / 2010$ & $2,123.11$ & 647.19 & 294.08 & 89.65 & $2,417.19$ & 736.84 \\
\hline 351828116371202 & $14 \mathrm{~N} / 3 \mathrm{E}-13 \mathrm{M} 3 \mathrm{~S}$ & $10 / 24 / 2011$ & $2,121.43$ & 646.68 & 295.76 & 90.16 & $2,417.19$ & 736.84 \\
\hline 351828116371202 & $14 \mathrm{~N} / 3 \mathrm{E}-13 \mathrm{M} 3 \mathrm{~S}$ & $10 / 30 / 2011$ & $2,121.65$ & 646.75 & 295.54 & 90.09 & $2,417.19$ & 736.84 \\
\hline 351828116371202 & $14 \mathrm{~N} / 3 \mathrm{E}-13 \mathrm{M} 3 \mathrm{~S}$ & $02 / 13 / 2012$ & $2,121.54$ & 646.72 & 295.65 & 90.12 & $2,417.19$ & 736.84 \\
\hline 351828116371202 & $14 \mathrm{~N} / 3 \mathrm{E}-13 \mathrm{M} 3 \mathrm{~S}$ & $02 / 21 / 2012$ & $2,120.78$ & 646.48 & 296.41 & 90.36 & $2,417.19$ & 736.84 \\
\hline 351828116371202 & $14 \mathrm{~N} / 3 \mathrm{E}-13 \mathrm{M} 3 \mathrm{~S}$ & $08 / 12 / 2014$ & $2,112.25$ & 643.88 & 304.94 & 92.96 & $2,417.19$ & 736.84 \\
\hline 351828116371202 & $14 \mathrm{~N} / 3 \mathrm{E}-13 \mathrm{M} 3 \mathrm{~S}$ & $11 / 06 / 2014$ & $2,112.99$ & 644.11 & 304.2 & 92.73 & $2,417.19$ & 736.84 \\
\hline 351828116371202 & $14 \mathrm{~N} / 3 \mathrm{E}-13 \mathrm{M} 3 \mathrm{~S}$ & $02 / 18 / 2015$ & $2,114.53$ & 644.58 & 302.66 & 92.26 & $2,417.19$ & 736.84 \\
\hline 351828116371202 & $14 \mathrm{~N} / 3 \mathrm{E}-13 \mathrm{M} 3 \mathrm{~S}$ & $05 / 20 / 2015$ & $2,117.34$ & 645.44 & 299.85 & 91.40 & $2,417.19$ & 736.84 \\
\hline 351828116371202 & $14 \mathrm{~N} / 3 \mathrm{E}-13 \mathrm{M} 3 \mathrm{~S}$ & $12 / 22 / 2015$ & $2,119.87$ & 646.21 & 297.32 & 90.63 & $2,417.19$ & 736.84 \\
\hline 351828116371202 & $14 \mathrm{~N} / 3 \mathrm{E}-13 \mathrm{M} 3 \mathrm{~S}$ & $05 / 24 / 2016$ & $2,114.28$ & 644.50 & 302.91 & 92.34 & $2,417.19$ & 736.84 \\
\hline 351828116371202 & $14 \mathrm{~N} / 3 \mathrm{E}-13 \mathrm{M} 3 \mathrm{~S}$ & 06/06/2016 & $2,114.16$ & 644.47 & 303.03 & 92.37 & $2,417.19$ & 736.84 \\
\hline 351828116371202 & $14 \mathrm{~N} / 3 \mathrm{E}-13 \mathrm{M} 3 \mathrm{~S}$ & $12 / 08 / 2016$ & $2,115.17$ & 644.77 & 302.02 & 92.07 & $2,417.19$ & 736.84 \\
\hline 351828116371202 & $14 \mathrm{~N} / 3 \mathrm{E}-13 \mathrm{M} 3 \mathrm{~S}$ & $12 / 08 / 2016$ & $2,115.13$ & 644.76 & 302.06 & 92.08 & $2,417.19$ & 736.84 \\
\hline 351828116371203 & $14 \mathrm{~N} / 3 \mathrm{E}-13 \mathrm{M} 4 \mathrm{~S}$ & 08/19/1997 & $2,114.81$ & 644.66 & 302.38 & 92.18 & $2,417.19$ & 736.84 \\
\hline 351828116371203 & $14 \mathrm{~N} / 3 \mathrm{E}-13 \mathrm{M} 4 \mathrm{~S}$ & 08/25/1997 & $2,115.17$ & 644.77 & 302.02 & 92.07 & $2,417.19$ & 736.84 \\
\hline 351828116371203 & $14 \mathrm{~N} / 3 \mathrm{E}-13 \mathrm{M} 4 \mathrm{~S}$ & 09/03/1997 & $2,113.76$ & 644.34 & 303.43 & 92.50 & $2,417.19$ & 736.84 \\
\hline 351828116371203 & $14 \mathrm{~N} / 3 \mathrm{E}-13 \mathrm{M} 4 \mathrm{~S}$ & 09/24/1997 & $2,113.79$ & 644.35 & 303.4 & 92.49 & $2,417.19$ & 736.84 \\
\hline 351828116371203 & $14 \mathrm{~N} / 3 \mathrm{E}-13 \mathrm{M} 4 \mathrm{~S}$ & 10/02/1997 & $2,113.36$ & 644.22 & 303.83 & 92.62 & $2,417.19$ & 736.84 \\
\hline 351828116371203 & $14 \mathrm{~N} / 3 \mathrm{E}-13 \mathrm{M} 4 \mathrm{~S}$ & 07/09/1999 & $2,106.59$ & 642.16 & 310.6 & 94.68 & $2,417.19$ & 736.84 \\
\hline 351828116371203 & $14 \mathrm{~N} / 3 \mathrm{E}-13 \mathrm{M} 4 \mathrm{~S}$ & 03/30/2000 & $2,119.05$ & 645.96 & 298.14 & 90.88 & $2,417.19$ & 736.84 \\
\hline 351828116371203 & $14 \mathrm{~N} / 3 \mathrm{E}-13 \mathrm{M} 4 \mathrm{~S}$ & $07 / 28 / 2000$ & $2,123.46$ & 647.30 & 293.73 & 89.54 & $2,417.19$ & 736.84 \\
\hline 351828116371203 & $14 \mathrm{~N} / 3 \mathrm{E}-13 \mathrm{M} 4 \mathrm{~S}$ & $01 / 30 / 2002$ & $2,103.18$ & 641.12 & 314.01 & 95.72 & $2,417.19$ & 736.84 \\
\hline 351828116371203 & $14 \mathrm{~N} / 3 \mathrm{E}-13 \mathrm{M} 4 \mathrm{~S}$ & 03/01/2005 & $2,123.4$ & 647.28 & 293.79 & 89.56 & $2,417.19$ & 736.84 \\
\hline 351828116371203 & $14 \mathrm{~N} / 3 \mathrm{E}-13 \mathrm{M} 4 \mathrm{~S}$ & $11 / 06 / 2007$ & $2,129.26$ & 649.07 & 287.93 & 87.77 & $2,417.19$ & 736.84 \\
\hline 351828116371203 & $14 \mathrm{~N} / 3 \mathrm{E}-13 \mathrm{M} 4 \mathrm{~S}$ & $12 / 19 / 2007$ & $2,129.36$ & 649.10 & 287.83 & 87.74 & $2,417.19$ & 736.84 \\
\hline 351828116371203 & $14 \mathrm{~N} / 3 \mathrm{E}-13 \mathrm{M} 4 \mathrm{~S}$ & $02 / 13 / 2008$ & $2,130.28$ & 649.38 & 286.91 & 87.46 & $2,417.19$ & 736.84 \\
\hline 351828116371203 & $14 \mathrm{~N} / 3 \mathrm{E}-13 \mathrm{M} 4 \mathrm{~S}$ & $04 / 29 / 2008$ & $2,130.45$ & 649.43 & 286.74 & 87.41 & $2,417.19$ & 736.84 \\
\hline
\end{tabular}


Appendix 2. Water-level data for selected wells in Bicycle Basin, Fort Irwin National Training Center, California, 19552017.-Continued

[State well No.: See Well-Numbering System in text. Abbreviations: ID, identification number; mm/dd/yyyy, month/day/year; NAVD 88, North American Vertical Datum of 1988, - , well is dry]

\begin{tabular}{|c|c|c|c|c|c|c|c|c|}
\hline \multirow[t]{2}{*}{ Site ID } & \multirow{2}{*}{$\begin{array}{c}\text { State } \\
\text { well number }\end{array}$} & \multirow{2}{*}{$\begin{array}{c}\text { Date } \\
\text { (mm/dd/yyyy) }\end{array}$} & \multicolumn{2}{|c|}{$\begin{array}{c}\text { Water-surface altitude, } \\
\text { above NAVD } 88\end{array}$} & \multicolumn{2}{|c|}{$\begin{array}{l}\text { Depth to water, below } \\
\text { land surface }\end{array}$} & \multicolumn{2}{|c|}{$\begin{array}{c}\text { Land-surface altitude, } \\
\text { above NAVD } 88^{1}\end{array}$} \\
\hline & & & (feet) & (meters) & (feet) & (meters) & (feet) & (meters) \\
\hline 351828116371203 & $14 \mathrm{~N} / 3 \mathrm{E}-13 \mathrm{M} 4 \mathrm{~S}$ & $07 / 29 / 2008$ & $2,130.48$ & 649.44 & 286.71 & 87.40 & $2,417.19$ & 736.84 \\
\hline 351828116371203 & $14 \mathrm{~N} / 3 \mathrm{E}-13 \mathrm{M} 4 \mathrm{~S}$ & $01 / 22 / 2009$ & $2,131.26$ & 649.68 & 285.93 & 87.16 & $2,417.19$ & 736.84 \\
\hline 351828116371203 & $14 \mathrm{~N} / 3 \mathrm{E}-13 \mathrm{M} 4 \mathrm{~S}$ & $10 / 07 / 2009$ & $2,125.15$ & 647.82 & 292.04 & 89.02 & $2,417.19$ & 736.84 \\
\hline 351828116371203 & $14 \mathrm{~N} / 3 \mathrm{E}-13 \mathrm{M} 4 \mathrm{~S}$ & $06 / 14 / 2010$ & $2,127.92$ & 648.66 & 289.27 & 88.18 & $2,417.19$ & 736.84 \\
\hline 351828116371203 & $14 \mathrm{~N} / 3 \mathrm{E}-13 \mathrm{M} 4 \mathrm{~S}$ & $12 / 13 / 2010$ & $2,122.34$ & 646.96 & 294.85 & 89.88 & $2,417.19$ & 736.84 \\
\hline 351828116371203 & $14 \mathrm{~N} / 3 \mathrm{E}-13 \mathrm{M} 4 \mathrm{~S}$ & $12 / 13 / 2010$ & $2,122.3$ & 646.95 & 294.89 & 89.89 & $2,417.19$ & 736.84 \\
\hline 351828116371203 & $14 \mathrm{~N} / 3 \mathrm{E}-13 \mathrm{M} 4 \mathrm{~S}$ & $02 / 13 / 2012$ & $2,120.46$ & 646.39 & 296.73 & 90.45 & $2,417.19$ & 736.84 \\
\hline 351828116371203 & $14 \mathrm{~N} / 3 \mathrm{E}-13 \mathrm{M} 4 \mathrm{~S}$ & $02 / 21 / 2012$ & $2,121.14$ & 646.59 & 296.05 & 90.25 & $2,417.19$ & 736.84 \\
\hline 351828116371203 & $14 \mathrm{~N} / 3 \mathrm{E}-13 \mathrm{M} 4 \mathrm{~S}$ & $08 / 30 / 2012$ & 2,115 & 644.72 & 302.19 & 92.12 & $2,417.19$ & 736.84 \\
\hline 351828116371203 & $14 \mathrm{~N} / 3 \mathrm{E}-13 \mathrm{M} 4 \mathrm{~S}$ & $04 / 17 / 2013$ & $2,116.96$ & 645.32 & 300.23 & 91.52 & $2,417.19$ & 736.84 \\
\hline 351828116371203 & $14 \mathrm{~N} / 3 \mathrm{E}-13 \mathrm{M} 4 \mathrm{~S}$ & 09/10/2013 & $2,115.49$ & 644.87 & 301.7 & 91.97 & $2,417.19$ & 736.84 \\
\hline 351828116371203 & $14 \mathrm{~N} / 3 \mathrm{E}-13 \mathrm{M} 4 \mathrm{~S}$ & $08 / 01 / 2014$ & $2,113.59$ & 644.29 & 303.6 & 92.55 & $2,417.19$ & 736.84 \\
\hline 351828116371203 & $14 \mathrm{~N} / 3 \mathrm{E}-13 \mathrm{M} 4 \mathrm{~S}$ & $11 / 06 / 2014$ & $2,112.74$ & 644.03 & 304.45 & 92.81 & $2,417.19$ & 736.84 \\
\hline 351828116371203 & $14 \mathrm{~N} / 3 \mathrm{E}-13 \mathrm{M} 4 \mathrm{~S}$ & $02 / 18 / 2015$ & $2,114.12$ & 644.45 & 303.07 & 92.39 & $2,417.19$ & 736.84 \\
\hline 351828116371203 & $14 \mathrm{~N} / 3 \mathrm{E}-13 \mathrm{M} 4 \mathrm{~S}$ & $05 / 20 / 2015$ & $2,117.16$ & 645.38 & 300.03 & 91.46 & $2,417.19$ & 736.84 \\
\hline 351828116371203 & $14 \mathrm{~N} / 3 \mathrm{E}-13 \mathrm{M} 4 \mathrm{~S}$ & 03/08/2017 & $2,116.73$ & 645.25 & 300.46 & 91.59 & $2,417.19$ & 736.84 \\
\hline 351828116371203 & $14 \mathrm{~N} / 3 \mathrm{E}-13 \mathrm{M} 4 \mathrm{~S}$ & $05 / 16 / 2017$ & $2,117.9$ & 645.61 & 299.29 & 91.23 & $2,417.19$ & 736.84 \\
\hline \multicolumn{9}{|c|}{ Local well ID: B-2 } \\
\hline 351830116372601 & $14 \mathrm{~N} / 3 \mathrm{E}-14 \mathrm{H} 1 \mathrm{~S}$ & $11 / 14 / 1964$ & $2,220.6$ & 676.91 & 202 & 61.58 & $2,422.6$ & 738.49 \\
\hline 351830116372601 & $14 \mathrm{~N} / 3 \mathrm{E}-14 \mathrm{H} 1 \mathrm{~S}$ & 03/01/1967 & $2,192.6$ & 668.38 & 230 & 70.11 & $2,422.6$ & 738.49 \\
\hline 351830116372601 & $14 \mathrm{~N} / 3 \mathrm{E}-14 \mathrm{H} 1 \mathrm{~S}$ & $01 / 01 / 1968$ & $2,193.6$ & 668.68 & 229 & 69.81 & $2,422.6$ & 738.49 \\
\hline 351830116372601 & $14 \mathrm{~N} / 3 \mathrm{E}-14 \mathrm{H} 1 \mathrm{~S}$ & $01 / 01 / 1969$ & $2,180.6$ & 664.72 & 242 & 73.77 & $2,422.6$ & 738.49 \\
\hline 351830116372601 & $14 \mathrm{~N} / 3 \mathrm{E}-14 \mathrm{H} 1 \mathrm{~S}$ & 03/01/1970 & $2,182.6$ & 665.33 & 240 & 73.16 & $2,422.6$ & 738.49 \\
\hline 351830116372601 & $14 \mathrm{~N} / 3 \mathrm{E}-14 \mathrm{H} 1 \mathrm{~S}$ & $02 / 01 / 1974$ & $2,185.6$ & 666.24 & 237 & 72.25 & $2,422.6$ & 738.49 \\
\hline 351830116372601 & $14 \mathrm{~N} / 3 \mathrm{E}-14 \mathrm{H} 1 \mathrm{~S}$ & 09/01/1978 & $2,169.6$ & 661.37 & 253 & 77.12 & $2,422.6$ & 738.49 \\
\hline 351830116372601 & $14 \mathrm{~N} / 3 \mathrm{E}-14 \mathrm{H} 1 \mathrm{~S}$ & $01 / 01 / 1981$ & $2,159.6$ & 658.32 & 263 & 80.17 & $2,422.6$ & 738.49 \\
\hline 351830116372601 & $14 \mathrm{~N} / 3 \mathrm{E}-14 \mathrm{H} 1 \mathrm{~S}$ & $01 / 26 / 1993$ & $2,123.4$ & 647.28 & 299.18 & 91.20 & $2,422.6$ & 738.49 \\
\hline 351830116372601 & $14 \mathrm{~N} / 3 \mathrm{E}-14 \mathrm{H} 1 \mathrm{~S}$ & $07 / 21 / 1994$ & $2,122.2$ & 646.92 & 300.44 & 91.58 & $2,422.6$ & 738.49 \\
\hline 351830116372601 & $14 \mathrm{~N} / 3 \mathrm{E}-14 \mathrm{H} 1 \mathrm{~S}$ & 09/08/1994 & $2,121.1$ & 646.58 & 301.53 & 91.92 & $2,422.6$ & 738.49 \\
\hline
\end{tabular}


Appendix 2. Water-level data for selected wells in Bicycle Basin, Fort Irwin National Training Center, California, 19552017.-Continued

[State well No.: See Well-Numbering System in text. Abbreviations: ID, identification number; mm/dd/yyyy, month/day/year; NAVD 88, North American Vertical Datum of $1988, \ldots$, well is dry]

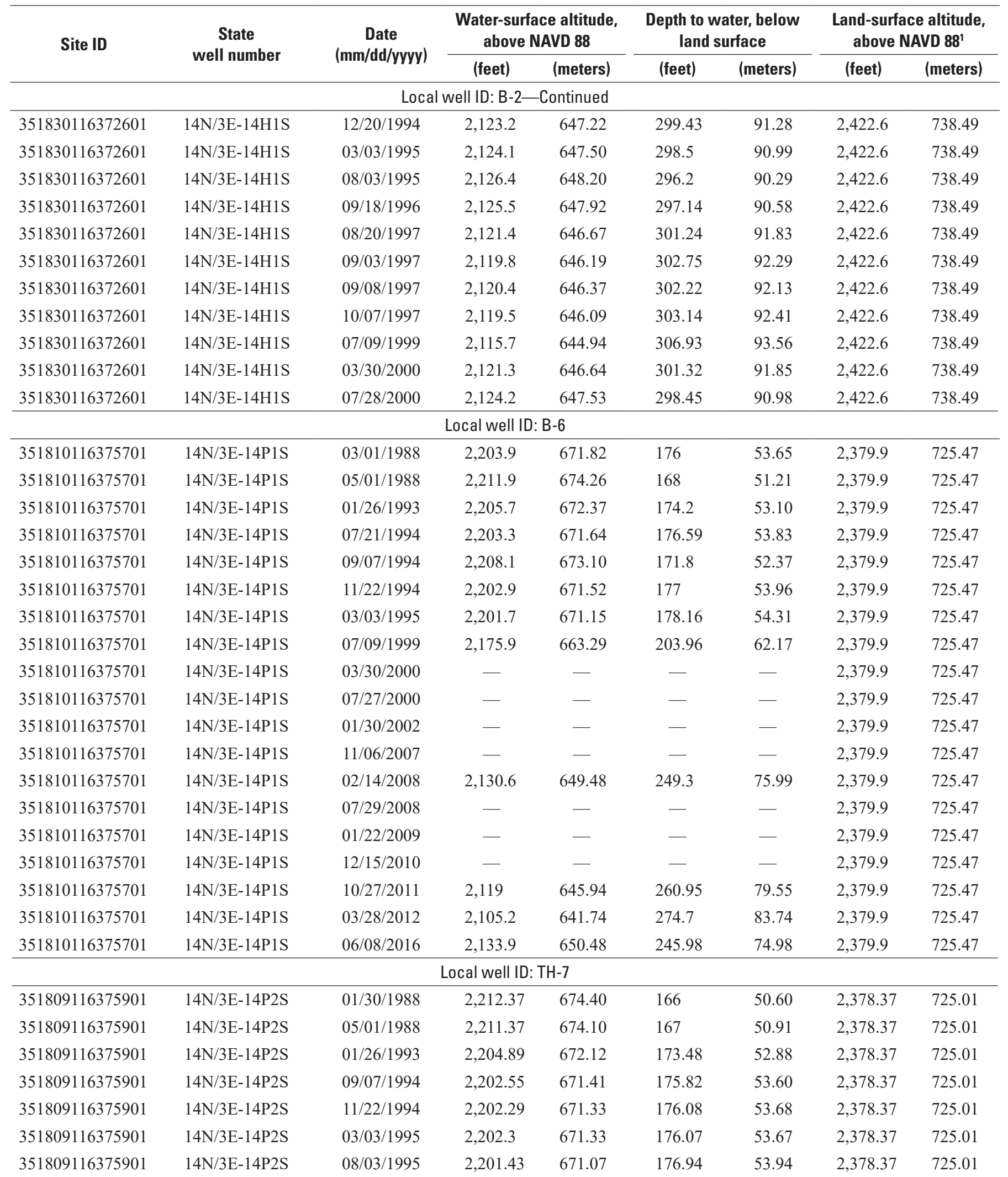


Appendix 2. Water-level data for selected wells in Bicycle Basin, Fort Irwin National Training Center, California, 19552017.-Continued

[State well No.: See Well-Numbering System in text. Abbreviations: ID, identification number; mm/dd/yyyy, month/day/year; NAVD 88, North American Vertical Datum of 1988, - , well is dry]

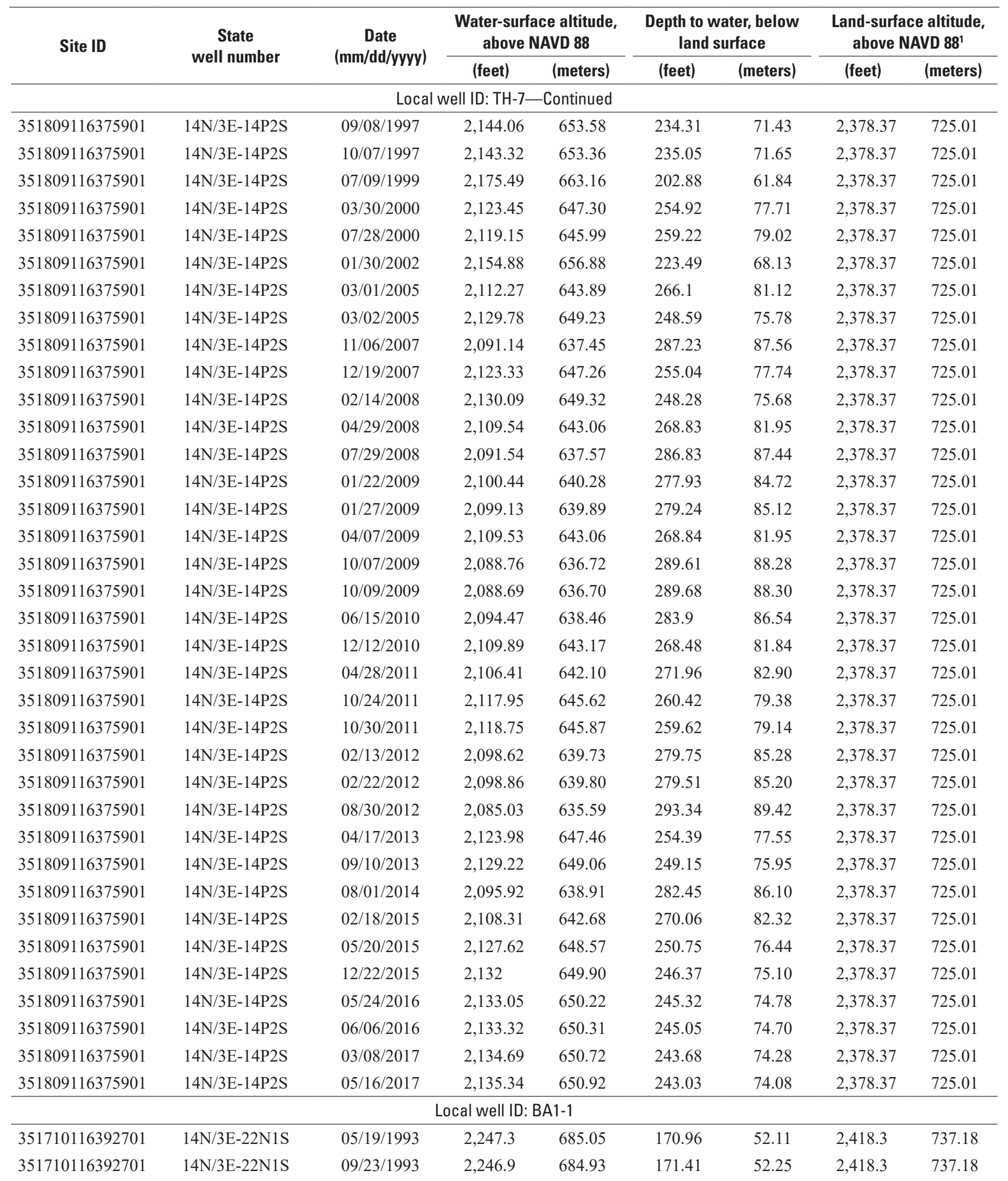


Appendix 2. Water-level data for selected wells in Bicycle Basin, Fort Irwin National Training Center, California, 19552017.-Continued

[State well No.: See Well-Numbering System in text. Abbreviations: ID, identification number; mm/dd/yyyy, month/day/year; NAVD 88, North American Vertical Datum of $1988, \ldots$, well is dry]

\begin{tabular}{|c|c|c|c|c|c|c|c|c|}
\hline \multirow{2}{*}{ Site ID } & \multirow{2}{*}{$\begin{array}{c}\text { State } \\
\text { well number }\end{array}$} & \multirow{2}{*}{$\begin{array}{c}\text { Date } \\
\text { (mm/dd/yyyy) }\end{array}$} & \multicolumn{2}{|c|}{$\begin{array}{c}\text { Water-surface altitude, } \\
\text { above NAVD } 88\end{array}$} & \multicolumn{2}{|c|}{$\begin{array}{l}\text { Depth to water, below } \\
\text { land surface }\end{array}$} & \multicolumn{2}{|c|}{$\begin{array}{c}\text { Land-surface altitude, } \\
\text { above NAVD } 88^{1}\end{array}$} \\
\hline & & & (feet) & (meters) & (feet) & (meters) & (feet) & (meters) \\
\hline 351710116392701 & $14 \mathrm{~N} / 3 \mathrm{E}-22 \mathrm{~N} 1 \mathrm{~S}$ & 07/21/1994 & $2,246.9$ & 684.93 & 171.45 & 52.26 & $2,418.3$ & 737.18 \\
\hline 351710116392701 & $14 \mathrm{~N} / 3 \mathrm{E}-22 \mathrm{~N} 1 \mathrm{~S}$ & 09/08/1994 & $2,246.7$ & 684.87 & 171.56 & 52.30 & $2,418.3$ & 737.18 \\
\hline 351710116392701 & $14 \mathrm{~N} / 3 \mathrm{E}-22 \mathrm{~N} 1 \mathrm{~S}$ & 03/04/1995 & $2,246.7$ & 684.87 & 171.6 & 52.31 & $2,418.3$ & 737.18 \\
\hline 351710116392701 & $14 \mathrm{~N} / 3 \mathrm{E}-22 \mathrm{~N} 1 \mathrm{~S}$ & 08/03/1995 & $2,246.7$ & 684.87 & 171.62 & 52.32 & $2,418.3$ & 737.18 \\
\hline 351710116392701 & $14 \mathrm{~N} / 3 \mathrm{E}-22 \mathrm{~N} 1 \mathrm{~S}$ & 05/22/1996 & $2,246.8$ & 684.90 & 171.54 & 52.29 & $2,418.3$ & 737.18 \\
\hline 351710116392701 & $14 \mathrm{~N} / 3 \mathrm{E}-22 \mathrm{~N} 1 \mathrm{~S}$ & 06/12/1996 & $2,246.7$ & 684.87 & 171.64 & 52.32 & $2,418.3$ & 737.18 \\
\hline \multicolumn{9}{|c|}{ Local well ID: B-3 } \\
\hline 351719116390301 & $14 \mathrm{~N} / 3 \mathrm{E}-22 \mathrm{P} 1 \mathrm{~S}$ & $01 / 26 / 1993$ & 2,243 & 683.74 & 189.18 & 57.67 & 2,432 & 741.35 \\
\hline 351719116390301 & $14 \mathrm{~N} / 3 \mathrm{E}-22 \mathrm{P} 1 \mathrm{~S}$ & 07/21/1994 & 2,242 & 683.44 & 189.81 & 57.86 & 2,432 & 741.35 \\
\hline 351719116390301 & $14 \mathrm{~N} / 3 \mathrm{E}-22 \mathrm{P} 1 \mathrm{~S}$ & 09/08/1994 & 2,242 & 683.44 & 190.23 & 57.99 & 2,432 & 741.35 \\
\hline 351719116390301 & $14 \mathrm{~N} / 3 \mathrm{E}-22 \mathrm{P} 1 \mathrm{~S}$ & $11 / 22 / 1994$ & 2,241 & 683.13 & 190.63 & 58.11 & 2,432 & 741.35 \\
\hline 351719116390301 & $14 \mathrm{~N} / 3 \mathrm{E}-22 \mathrm{P} 1 \mathrm{~S}$ & 03/04/1995 & 2,242 & 683.44 & 190.42 & 58.05 & 2,432 & 741.35 \\
\hline 351719116390301 & $14 \mathrm{~N} / 3 \mathrm{E}-22 \mathrm{P} 1 \mathrm{~S}$ & 08/03/1995 & 2,241 & 683.13 & 190.67 & 58.12 & 2,432 & 741.35 \\
\hline 351719116390301 & $14 \mathrm{~N} / 3 \mathrm{E}-22 \mathrm{P} 1 \mathrm{~S}$ & 09/18/1996 & 2,240 & 682.83 & 191.51 & 58.38 & 2,432 & 741.35 \\
\hline \multicolumn{9}{|c|}{ Local well ID: BLA4-1 } \\
\hline 351759116374401 & $14 \mathrm{~N} / 3 \mathrm{E}-23 \mathrm{~B} 1 \mathrm{~S}$ & $10 / 08 / 2009$ & $2,134.4$ & 650.64 & 240.72 & 73.38 & $2,375.12$ & 724.02 \\
\hline 351759116374401 & $14 \mathrm{~N} / 3 \mathrm{E}-23 \mathrm{~B} 1 \mathrm{~S}$ & $03 / 02 / 2010$ & $2,142.64$ & 653.15 & 232.48 & 70.87 & $2,375.12$ & 724.02 \\
\hline 351759116374401 & $14 \mathrm{~N} / 3 \mathrm{E}-23 \mathrm{~B} 1 \mathrm{~S}$ & 03/02/2010 & $2,142.6$ & 653.14 & 232.52 & 70.88 & $2,375.12$ & 724.02 \\
\hline 351759116374401 & $14 \mathrm{~N} / 3 \mathrm{E}-23 \mathrm{~B} 1 \mathrm{~S}$ & $06 / 15 / 2010$ & $2,140.39$ & 652.46 & 234.73 & 71.55 & $2,375.12$ & 724.02 \\
\hline 351759116374401 & $14 \mathrm{~N} / 3 \mathrm{E}-23 \mathrm{~B} 1 \mathrm{~S}$ & $12 / 14 / 2010$ & $2,134.54$ & 650.68 & 240.58 & 73.34 & $2,375.12$ & 724.02 \\
\hline 351759116374401 & $14 \mathrm{~N} / 3 \mathrm{E}-23 \mathrm{~B} 1 \mathrm{~S}$ & $12 / 14 / 2010$ & $2,134.61$ & 650.70 & 240.51 & 73.32 & $2,375.12$ & 724.02 \\
\hline 351759116374401 & $14 \mathrm{~N} / 3 \mathrm{E}-23 \mathrm{~B} 1 \mathrm{~S}$ & $12 / 17 / 2010$ & $2,134.51$ & 650.67 & 240.61 & 73.35 & $2,375.12$ & 724.02 \\
\hline 351759116374401 & $14 \mathrm{~N} / 3 \mathrm{E}-23 \mathrm{~B} 1 \mathrm{~S}$ & $12 / 17 / 2010$ & $2,134.47$ & 650.66 & 240.65 & 73.36 & $2,375.12$ & 724.02 \\
\hline 351759116374401 & $14 \mathrm{~N} / 3 \mathrm{E}-23 \mathrm{~B} 1 \mathrm{~S}$ & $04 / 28 / 2011$ & $2,133.61$ & 650.40 & 241.51 & 73.62 & $2,375.12$ & 724.02 \\
\hline 351759116374401 & $14 \mathrm{~N} / 3 \mathrm{E}-23 \mathrm{~B} 1 \mathrm{~S}$ & $10 / 25 / 2011$ & $2,133.81$ & 650.46 & 241.31 & 73.56 & $2,375.12$ & 724.02 \\
\hline 351759116374401 & $14 \mathrm{~N} / 3 \mathrm{E}-23 \mathrm{~B} 1 \mathrm{~S}$ & $10 / 30 / 2011$ & $2,133.96$ & 650.50 & 241.16 & 73.51 & $2,375.12$ & 724.02 \\
\hline 351759116374401 & $14 \mathrm{~N} / 3 \mathrm{E}-23 \mathrm{~B} 1 \mathrm{~S}$ & $02 / 13 / 2012$ & $2,137.26$ & 651.51 & 237.86 & 72.51 & $2,375.12$ & 724.02 \\
\hline 351759116374401 & $14 \mathrm{~N} / 3 \mathrm{E}-23 \mathrm{~B} 1 \mathrm{~S}$ & $02 / 22 / 2012$ & $2,136.91$ & 651.40 & 238.21 & 72.61 & $2,375.12$ & 724.02 \\
\hline 351759116374401 & $14 \mathrm{~N} / 3 \mathrm{E}-23 \mathrm{~B} 1 \mathrm{~S}$ & 08/30/2012 & $2,129.27$ & 649.07 & 245.85 & 74.94 & $2,375.12$ & 724.02 \\
\hline
\end{tabular}


Appendix 2. Water-level data for selected wells in Bicycle Basin, Fort Irwin National Training Center, California, 19552017.-Continued

[State well No.: See Well-Numbering System in text. Abbreviations: ID, identification number; mm/dd/yyyy, month/day/year; NAVD 88, North American Vertical Datum of $1988,-$, well is dry]

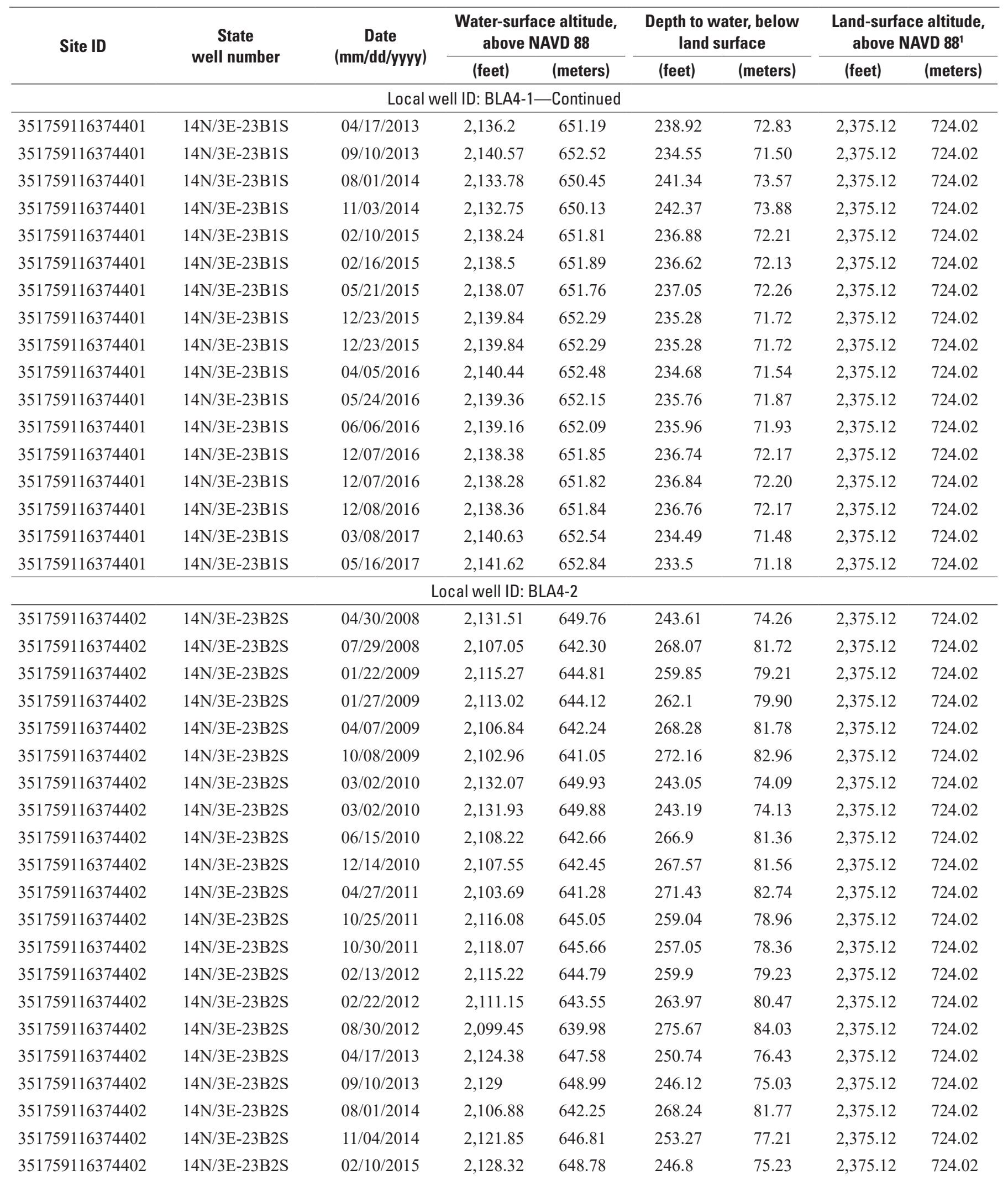


Appendix 2. Water-level data for selected wells in Bicycle Basin, Fort Irwin National Training Center, California, 19552017.-Continued

[State well No.: See Well-Numbering System in text. Abbreviations: ID, identification number; mm/dd/yyyy, month/day/year; NAVD 88, North American Vertical Datum of 1988, -, well is dry]

\begin{tabular}{|c|c|c|c|c|c|c|c|c|}
\hline \multirow[t]{2}{*}{ Site ID } & \multirow{2}{*}{$\begin{array}{c}\text { State } \\
\text { well number }\end{array}$} & \multirow{2}{*}{$\begin{array}{c}\text { Date } \\
\text { (mm/dd/yyyy) }\end{array}$} & \multicolumn{2}{|c|}{$\begin{array}{c}\text { Water-surface altitude, } \\
\text { above NAVD } 88\end{array}$} & \multicolumn{2}{|c|}{$\begin{array}{l}\text { Depth to water, below } \\
\text { land surface }\end{array}$} & \multicolumn{2}{|c|}{$\begin{array}{c}\text { Land-surface altitude, } \\
\text { above NAVD } 88^{1}\end{array}$} \\
\hline & & & (feet) & (meters) & (feet) & (meters) & (feet) & (meters) \\
\hline 351759116374402 & $14 \mathrm{~N} / 3 \mathrm{E}-23 \mathrm{~B} 2 \mathrm{~S}$ & $02 / 16 / 2015$ & $2,118.65$ & 645.84 & 256.47 & 78.18 & $2,375.12$ & 724.02 \\
\hline 351759116374402 & $14 \mathrm{~N} / 3 \mathrm{E}-23 \mathrm{~B} 2 \mathrm{~S}$ & $02 / 17 / 2015$ & $2,117.96$ & 645.63 & 257.16 & 78.39 & $2,375.12$ & 724.02 \\
\hline 351759116374402 & $14 \mathrm{~N} / 3 \mathrm{E}-23 \mathrm{~B} 2 \mathrm{~S}$ & $12 / 23 / 2015$ & $2,132.02$ & 649.91 & 243.1 & 74.11 & $2,375.12$ & 724.02 \\
\hline 351759116374402 & $14 \mathrm{~N} / 3 \mathrm{E}-23 \mathrm{~B} 2 \mathrm{~S}$ & $05 / 24 / 2016$ & $2,133.08$ & 650.23 & 242.04 & 73.78 & $2,375.12$ & 724.02 \\
\hline 351759116374402 & $14 \mathrm{~N} / 3 \mathrm{E}-23 \mathrm{~B} 2 \mathrm{~S}$ & $06 / 06 / 2016$ & $2,133.23$ & 650.28 & 241.89 & 73.74 & $2,375.12$ & 724.02 \\
\hline 351759116374402 & $14 \mathrm{~N} / 3 \mathrm{E}-23 \mathrm{~B} 2 \mathrm{~S}$ & $06 / 29 / 2016$ & $2,133.31$ & 650.30 & 241.81 & 73.71 & $2,375.12$ & 724.02 \\
\hline 351759116374402 & $14 \mathrm{~N} / 3 \mathrm{E}-23 \mathrm{~B} 2 \mathrm{~S}$ & $05 / 16 / 2017$ & $2,135.34$ & 650.92 & 239.78 & 73.09 & $2,375.12$ & 724.02 \\
\hline \multicolumn{9}{|c|}{ Local well ID: BLA4-3 } \\
\hline 351759116374403 & $14 \mathrm{~N} / 3 \mathrm{E}-23 \mathrm{~B} 3 \mathrm{~S}$ & 04/30/2008 & $2,143.48$ & 653.40 & 231.64 & 70.61 & $2,375.12$ & 724.02 \\
\hline 351759116374403 & $14 \mathrm{~N} / 3 \mathrm{E}-23 \mathrm{~B} 3 \mathrm{~S}$ & 07/29/2008 & $2,141.95$ & 652.94 & 233.17 & 71.08 & $2,375.12$ & 724.02 \\
\hline 351759116374403 & $14 \mathrm{~N} / 3 \mathrm{E}-23 \mathrm{~B} 3 \mathrm{~S}$ & $01 / 22 / 2009$ & $2,142.1$ & 652.98 & 233.02 & 71.03 & $2,375.12$ & 724.02 \\
\hline 351759116374403 & $14 \mathrm{~N} / 3 \mathrm{E}-23 \mathrm{~B} 3 \mathrm{~S}$ & 01/27/2009 & $2,141.61$ & 652.83 & 233.51 & 71.18 & $2,375.12$ & 724.02 \\
\hline 351759116374403 & $14 N / 3 E-23 B 3 S$ & 04/07/2009 & $2,141.17$ & 652.70 & 233.95 & 71.32 & $2,375.12$ & 724.02 \\
\hline 351759116374403 & $14 \mathrm{~N} / 3 \mathrm{E}-23 \mathrm{~B} 3 \mathrm{~S}$ & $10 / 08 / 2009$ & $2,140.08$ & 652.37 & 235.04 & 71.65 & $2,375.12$ & 724.02 \\
\hline 351759116374403 & $14 \mathrm{~N} / 3 \mathrm{E}-23 \mathrm{~B} 3 \mathrm{~S}$ & 03/02/2010 & $2,141.11$ & 652.68 & 234.01 & 71.33 & $2,375.12$ & 724.02 \\
\hline 351759116374403 & $14 \mathrm{~N} / 3 \mathrm{E}-23 \mathrm{~B} 3 \mathrm{~S}$ & $10 / 25 / 2011$ & $2,137.84$ & 651.69 & 237.28 & 72.33 & $2,375.12$ & 724.02 \\
\hline 351759116374403 & $14 \mathrm{~N} / 3 \mathrm{E}-23 \mathrm{~B} 3 \mathrm{~S}$ & $10 / 30 / 2011$ & $2,137.7$ & 651.64 & 237.42 & 72.37 & $2,375.12$ & 724.02 \\
\hline 351759116374403 & $14 \mathrm{~N} / 3 \mathrm{E}-23 \mathrm{~B} 3 \mathrm{~S}$ & 02/13/2012 & $2,138.1$ & 651.76 & 237.02 & 72.25 & $2,375.12$ & 724.02 \\
\hline 351759116374403 & $14 N / 3 E-23 B 3 S$ & $02 / 22 / 2012$ & $2,137.47$ & 651.57 & 237.65 & 72.44 & $2,375.12$ & 724.02 \\
\hline 351759116374403 & $14 N / 3 E-23 B 3 S$ & $08 / 30 / 2012$ & $2,136.25$ & 651.20 & 238.87 & 72.82 & $2,375.12$ & 724.02 \\
\hline 351759116374403 & $14 \mathrm{~N} / 3 \mathrm{E}-23 \mathrm{~B} 3 \mathrm{~S}$ & $04 / 17 / 2013$ & $2,135.72$ & 651.04 & 239.4 & 72.98 & $2,375.12$ & 724.02 \\
\hline 351759116374403 & $14 \mathrm{~N} / 3 \mathrm{E}-23 \mathrm{~B} 3 \mathrm{~S}$ & $09 / 10 / 2013$ & $2,136.78$ & 651.36 & 238.34 & 72.65 & $2,375.12$ & 724.02 \\
\hline 351759116374403 & $14 \mathrm{~N} / 3 \mathrm{E}-23 \mathrm{~B} 3 \mathrm{~S}$ & 08/01/2014 & $2,135.99$ & 651.12 & 239.13 & 72.89 & $2,375.12$ & 724.02 \\
\hline 351759116374403 & $14 N / 3 E-23 B 3 S$ & $11 / 03 / 2014$ & $2,135.33$ & 650.92 & 239.79 & 73.10 & $2,375.12$ & 724.02 \\
\hline 351759116374403 & $14 N / 3 E-23 B 3 S$ & $02 / 10 / 2015$ & $2,136.08$ & 651.15 & 239.04 & 72.87 & $2,375.12$ & 724.02 \\
\hline 351759116374403 & $14 \mathrm{~N} / 3 \mathrm{E}-23 \mathrm{~B} 3 \mathrm{~S}$ & $02 / 15 / 2015$ & $2,136.09$ & 651.15 & 239.03 & 72.86 & $2,375.12$ & 724.02 \\
\hline 351759116374403 & $14 \mathrm{~N} / 3 \mathrm{E}-23 \mathrm{~B} 3 \mathrm{~S}$ & $02 / 17 / 2015$ & $2,135.9$ & 651.09 & 239.22 & 72.92 & $2,375.12$ & 724.02 \\
\hline 351759116374403 & $14 \mathrm{~N} / 3 \mathrm{E}-23 \mathrm{~B} 3 \mathrm{~S}$ & $05 / 20 / 2015$ & $2,135.97$ & 651.12 & 239.15 & 72.90 & $2,375.12$ & 724.02 \\
\hline 351759116374403 & $14 \mathrm{~N} / 3 \mathrm{E}-23 \mathrm{~B} 3 \mathrm{~S}$ & $12 / 23 / 2015$ & $2,137.08$ & 651.45 & 238.04 & 72.56 & $2,375.12$ & 724.02 \\
\hline
\end{tabular}


Appendix 2. Water-level data for selected wells in Bicycle Basin, Fort Irwin National Training Center, California, 19552017.-Continued

[State well No.: See Well-Numbering System in text. Abbreviations: ID, identification number; mm/dd/yyyy, month/day/year; NAVD 88, North American Vertical Datum of $1988, \ldots$, well is dry]

\begin{tabular}{|c|c|c|c|c|c|c|c|c|}
\hline \multirow[t]{2}{*}{ Site ID } & \multirow{2}{*}{$\begin{array}{c}\text { State } \\
\text { well number }\end{array}$} & \multirow{2}{*}{$\begin{array}{c}\text { Date } \\
\text { (mm/dd/yyyy) }\end{array}$} & \multicolumn{2}{|c|}{$\begin{array}{c}\text { Water-surface altitude, } \\
\text { above NAVD } 88\end{array}$} & \multicolumn{2}{|c|}{$\begin{array}{c}\text { Depth to water, below } \\
\text { land surface }\end{array}$} & \multicolumn{2}{|c|}{$\begin{array}{l}\text { Land-surface altitude } \\
\text { above NAVD 88' }\end{array}$} \\
\hline & & & (feet) & (meters) & (feet) & (meters) & (feet) & (meters) \\
\hline 351759116374403 & $14 \mathrm{~N} / 3 \mathrm{E}-23 \mathrm{~B} 3 \mathrm{~S}$ & $05 / 24 / 2016$ & $2,137.16$ & 651.48 & 237.96 & 72.54 & $2,375.12$ & 724.02 \\
\hline 351759116374403 & $14 \mathrm{~N} / 3 \mathrm{E}-23 \mathrm{~B} 3 \mathrm{~S}$ & $06 / 06 / 2016$ & $2,137.33$ & 651.53 & 237.79 & 72.49 & $2,375.12$ & 724.02 \\
\hline 351759116374403 & $14 \mathrm{~N} / 3 \mathrm{E}-23 \mathrm{~B} 3 \mathrm{~S}$ & $12 / 07 / 2016$ & $2,137.47$ & 651.57 & 237.65 & 72.44 & $2,375.12$ & 724.02 \\
\hline 351759116374403 & $14 \mathrm{~N} / 3 \mathrm{E}-23 \mathrm{~B} 3 \mathrm{~S}$ & $12 / 08 / 2016$ & $2,137.34$ & 651.53 & 237.78 & 72.48 & $2,375.12$ & 724.02 \\
\hline 351759116374403 & $14 \mathrm{~N} / 3 \mathrm{E}-23 \mathrm{~B} 3 \mathrm{~S}$ & 03/08/2017 & $2,137.48$ & 651.58 & 237.64 & 72.44 & $2,375.12$ & 724.02 \\
\hline 351759116374403 & $14 \mathrm{~N} / 3 \mathrm{E}-23 \mathrm{~B} 3 \mathrm{~S}$ & $05 / 16 / 2017$ & $2,137.79$ & 651.67 & 237.33 & 72.35 & $2,375.12$ & 724.02 \\
\hline \multicolumn{9}{|c|}{ Local well ID: BX-2 } \\
\hline 351738116374101 & $14 \mathrm{~N} / 3 \mathrm{E}-23 \mathrm{G} 1 \mathrm{~S}$ & $09 / 24 / 1993$ & $2,213.26$ & 674.68 & 146.47 & 44.65 & $2,359.73$ & 719.32 \\
\hline 351738116374101 & $14 \mathrm{~N} / 3 \mathrm{E}-23 \mathrm{G} 1 \mathrm{~S}$ & $07 / 21 / 1994$ & $2,212.62$ & 674.48 & 147.11 & 44.84 & $2,359.73$ & 719.32 \\
\hline 351738116374101 & $14 \mathrm{~N} / 3 \mathrm{E}-23 \mathrm{G} 1 \mathrm{~S}$ & 09/08/1994 & $2,212.5$ & 674.44 & 147.23 & 44.88 & $2,359.73$ & 719.32 \\
\hline 351738116374101 & $14 \mathrm{~N} / 3 \mathrm{E}-23 \mathrm{G} 1 \mathrm{~S}$ & $09 / 21 / 1994$ & $2,212.37$ & 674.40 & 147.36 & 44.92 & $2,359.73$ & 719.32 \\
\hline 351738116374101 & $14 \mathrm{~N} / 3 \mathrm{E}-23 \mathrm{G} 1 \mathrm{~S}$ & $11 / 22 / 1994$ & $2,211.95$ & 674.28 & 147.78 & 45.05 & $2,359.73$ & 719.32 \\
\hline 351738116374101 & $14 \mathrm{~N} / 3 \mathrm{E}-23 \mathrm{G} 1 \mathrm{~S}$ & $02 / 02 / 1995$ & $2,212.16$ & 674.34 & 147.57 & 44.98 & $2,359.73$ & 719.32 \\
\hline 351738116374101 & $14 \mathrm{~N} / 3 \mathrm{E}-23 \mathrm{G} 1 \mathrm{~S}$ & 03/03/1995 & $2,212.01$ & 674.29 & 147.72 & 45.03 & $2,359.73$ & 719.32 \\
\hline 351738116374101 & $14 \mathrm{~N} / 3 \mathrm{E}-23 \mathrm{G} 1 \mathrm{~S}$ & 09/18/1996 & $2,210.16$ & 673.73 & 149.57 & 45.59 & $2,359.73$ & 719.32 \\
\hline 351738116374101 & $14 \mathrm{~N} / 3 \mathrm{E}-23 \mathrm{G} 1 \mathrm{~S}$ & 07/08/1997 & $2,209.2$ & 673.44 & 150.53 & 45.89 & $2,359.73$ & 719.32 \\
\hline 351738116374101 & $14 \mathrm{~N} / 3 \mathrm{E}-23 \mathrm{G} 1 \mathrm{~S}$ & $08 / 01 / 2000$ & $2,199.53$ & 670.49 & 160.2 & 48.83 & $2,359.73$ & 719.32 \\
\hline 351738116374101 & $14 \mathrm{~N} / 3 \mathrm{E}-23 \mathrm{G} 1 \mathrm{~S}$ & $01 / 30 / 2002$ & $2,194.44$ & 668.94 & 165.29 & 50.39 & $2,359.73$ & 719.32 \\
\hline 351738116374101 & $14 \mathrm{~N} / 3 \mathrm{E}-23 \mathrm{G} 1 \mathrm{~S}$ & $03 / 01 / 2005$ & $2,185.21$ & 666.13 & 174.52 & 53.20 & $2,359.73$ & 719.32 \\
\hline 351738116374101 & $14 \mathrm{~N} / 3 \mathrm{E}-23 \mathrm{G} 1 \mathrm{~S}$ & $11 / 06 / 2007$ & $2,178.9$ & 664.20 & 180.83 & 55.12 & $2,359.73$ & 719.32 \\
\hline 351738116374101 & $14 \mathrm{~N} / 3 \mathrm{E}-23 \mathrm{G} 1 \mathrm{~S}$ & $12 / 18 / 2007$ & $2,178.61$ & 664.11 & 181.12 & 55.21 & $2,359.73$ & 719.32 \\
\hline 351738116374101 & $14 \mathrm{~N} / 3 \mathrm{E}-23 \mathrm{G} 1 \mathrm{~S}$ & $02 / 15 / 2008$ & $2,178.15$ & 663.97 & 181.58 & 55.35 & $2,359.73$ & 719.32 \\
\hline 351738116374101 & $14 \mathrm{~N} / 3 \mathrm{E}-23 \mathrm{G} 1 \mathrm{~S}$ & $04 / 29 / 2008$ & $2,177.95$ & 663.91 & 181.78 & 55.41 & $2,359.73$ & 719.32 \\
\hline 351738116374101 & $14 \mathrm{~N} / 3 \mathrm{E}-23 \mathrm{G} 1 \mathrm{~S}$ & $07 / 29 / 2008$ & $2,176.83$ & 663.57 & 182.9 & 55.75 & $2,359.73$ & 719.32 \\
\hline 351738116374101 & $14 \mathrm{~N} / 3 \mathrm{E}-23 \mathrm{G} 1 \mathrm{~S}$ & $01 / 22 / 2009$ & $2,175.75$ & 663.24 & 183.98 & 56.08 & $2,359.73$ & 719.32 \\
\hline 351738116374101 & $14 \mathrm{~N} / 3 \mathrm{E}-23 \mathrm{G} 1 \mathrm{~S}$ & $04 / 07 / 2009$ & $2,175.08$ & 663.04 & 184.65 & 56.29 & $2,359.73$ & 719.32 \\
\hline 351738116374101 & $14 \mathrm{~N} / 3 \mathrm{E}-23 \mathrm{G} 1 \mathrm{~S}$ & $10 / 07 / 2009$ & $2,173.54$ & 662.57 & 186.19 & 56.76 & $2,359.73$ & 719.32 \\
\hline 351738116374101 & $14 \mathrm{~N} / 3 \mathrm{E}-23 \mathrm{G} 1 \mathrm{~S}$ & $06 / 14 / 2010$ & $2,172.08$ & 662.12 & 187.65 & 57.20 & $2,359.73$ & 719.32 \\
\hline 351738116374101 & $14 \mathrm{~N} / 3 \mathrm{E}-23 \mathrm{G} 1 \mathrm{~S}$ & $12 / 14 / 2010$ & $2,170.85$ & 661.75 & 188.88 & 57.58 & $2,359.73$ & 719.32 \\
\hline 351738116374101 & $14 \mathrm{~N} / 3 \mathrm{E}-23 \mathrm{G} 1 \mathrm{~S}$ & $12 / 14 / 2010$ & $2,170.98$ & 661.79 & 188.75 & 57.54 & $2,359.73$ & 719.32 \\
\hline
\end{tabular}


Appendix 2. Water-level data for selected wells in Bicycle Basin, Fort Irwin National Training Center, California, 19552017.-Continued

[State well No.: See Well-Numbering System in text. Abbreviations: ID, identification number; mm/dd/yyyy, month/day/year; NAVD 88, North American Vertical Datum of $1988, \ldots$, well is dry]

\begin{tabular}{|c|c|c|c|c|c|c|c|c|}
\hline \multirow[t]{2}{*}{ Site ID } & \multirow{2}{*}{$\begin{array}{c}\text { State } \\
\text { well number }\end{array}$} & \multirow{2}{*}{$\begin{array}{c}\text { Date } \\
\text { (mm/dd/yyyy) }\end{array}$} & \multicolumn{2}{|c|}{$\begin{array}{c}\text { Water-surface altitude, } \\
\text { above NAVD } 88\end{array}$} & \multicolumn{2}{|c|}{$\begin{array}{l}\text { Depth to water, below } \\
\text { land surface }\end{array}$} & \multicolumn{2}{|c|}{$\begin{array}{l}\text { Land-surface altitude, } \\
\text { above NAVD } 88^{1}\end{array}$} \\
\hline & & & (feet) & (meters) & (feet) & (meters) & (feet) & (meters) \\
\hline 351738116374101 & $14 \mathrm{~N} / 3 \mathrm{E}-23 \mathrm{G} 1 \mathrm{~S}$ & $04 / 28 / 2011$ & $2,169.98$ & 661.48 & 189.75 & 57.84 & $2,359.73$ & 719.32 \\
\hline 351738116374101 & $14 \mathrm{~N} / 3 \mathrm{E}-23 \mathrm{G} 1 \mathrm{~S}$ & $10 / 24 / 2011$ & $2,169.38$ & 661.30 & 190.35 & 58.03 & $2,359.73$ & 719.32 \\
\hline 351738116374101 & $14 \mathrm{~N} / 3 \mathrm{E}-23 \mathrm{G} 1 \mathrm{~S}$ & $02 / 22 / 2012$ & $2,168.67$ & 661.08 & 191.06 & 58.24 & $2,359.73$ & 719.32 \\
\hline 351738116374101 & $14 \mathrm{~N} / 3 \mathrm{E}-23 \mathrm{G} 1 \mathrm{~S}$ & $08 / 30 / 2012$ & $2,168.09$ & 660.91 & 191.64 & 58.42 & $2,359.73$ & 719.32 \\
\hline 351738116374101 & $14 \mathrm{~N} / 3 \mathrm{E}-23 \mathrm{G} 1 \mathrm{~S}$ & $04 / 17 / 2013$ & $2,167.07$ & 660.60 & 192.66 & 58.73 & $2,359.73$ & 719.32 \\
\hline 351738116374101 & $14 \mathrm{~N} / 3 \mathrm{E}-23 \mathrm{G} 1 \mathrm{~S}$ & 09/10/2013 & $2,166.96$ & 660.56 & 192.77 & 58.76 & $2,359.73$ & 719.32 \\
\hline 351738116374101 & $14 \mathrm{~N} / 3 \mathrm{E}-23 \mathrm{G} 1 \mathrm{~S}$ & $05 / 20 / 2015$ & $2,165.49$ & 660.11 & 194.24 & 59.21 & $2,359.73$ & 719.32 \\
\hline 351738116374101 & $14 \mathrm{~N} / 3 \mathrm{E}-23 \mathrm{G} 1 \mathrm{~S}$ & 09/30/2015 & $2,165.22$ & 660.03 & 194.51 & 59.29 & $2,359.73$ & 719.32 \\
\hline 351738116374101 & $14 \mathrm{~N} / 3 \mathrm{E}-23 \mathrm{G} 1 \mathrm{~S}$ & $12 / 23 / 2015$ & $2,165.39$ & 660.08 & 194.34 & 59.24 & $2,359.73$ & 719.32 \\
\hline 351738116374101 & $14 \mathrm{~N} / 3 \mathrm{E}-23 \mathrm{G} 1 \mathrm{~S}$ & $05 / 26 / 2016$ & $2,164.74$ & 659.89 & 194.99 & 59.44 & $2,359.73$ & 719.32 \\
\hline 351738116374101 & $14 \mathrm{~N} / 3 \mathrm{E}-23 \mathrm{G} 1 \mathrm{~S}$ & 06/06/2016 & $2,164.97$ & 659.96 & 194.76 & 59.37 & $2,359.73$ & 719.32 \\
\hline 351738116374101 & $14 \mathrm{~N} / 3 \mathrm{E}-23 \mathrm{G} 1 \mathrm{~S}$ & 03/08/2017 & $2,164.24$ & 659.73 & 195.49 & 59.59 & $2,359.73$ & 719.32 \\
\hline 351738116374101 & $14 \mathrm{~N} / 3 \mathrm{E}-23 \mathrm{G} 1 \mathrm{~S}$ & 05/16/2017 & $2,164.25$ & 659.74 & 195.48 & 59.59 & $2,359.73$ & 719.32 \\
\hline \multicolumn{9}{|c|}{ Local well ID: BX-1 } \\
\hline 351742116362401 & $14 \mathrm{~N} / 3 \mathrm{E}-24 \mathrm{H} 1 \mathrm{~S}$ & $10 / 30 / 1980$ & $2,218.9$ & 676.39 & 142.7 & 43.50 & $2,361.6$ & 719.89 \\
\hline 351742116362401 & $14 \mathrm{~N} / 3 \mathrm{E}-24 \mathrm{H} 1 \mathrm{~S}$ & 09/19/1994 & $2,189.3$ & 667.37 & 172.27 & 52.51 & $2,361.6$ & 719.89 \\
\hline 351742116362401 & $14 \mathrm{~N} / 3 \mathrm{E}-24 \mathrm{H} 1 \mathrm{~S}$ & $12 / 20 / 1994$ & $2,189.4$ & 667.40 & 172.18 & 52.49 & $2,361.6$ & 719.89 \\
\hline 351742116362401 & $14 \mathrm{~N} / 3 \mathrm{E}-24 \mathrm{H} 1 \mathrm{~S}$ & $01 / 31 / 1995$ & $2,189.8$ & 667.52 & 171.84 & 52.38 & $2,361.6$ & 719.89 \\
\hline 351742116362401 & $14 \mathrm{~N} / 3 \mathrm{E}-24 \mathrm{H} 1 \mathrm{~S}$ & 03/03/1995 & $2,189.6$ & 667.46 & 171.99 & 52.43 & $2,361.6$ & 719.89 \\
\hline 351742116362401 & $14 \mathrm{~N} / 3 \mathrm{E}-24 \mathrm{H} 1 \mathrm{~S}$ & 08/03/1995 & $2,188.5$ & 667.13 & 173.13 & 52.78 & $2,361.6$ & 719.89 \\
\hline 351742116362401 & $14 \mathrm{~N} / 3 \mathrm{E}-24 \mathrm{H} 1 \mathrm{~S}$ & 09/18/1996 & 2,183 & 665.45 & 178.57 & 54.43 & $2,361.6$ & 719.89 \\
\hline \multicolumn{9}{|c|}{ Local well ID: BLA3-1 } \\
\hline 351716116363701 & 14N/3E-24Q1S & 07/14/1997 & $2,150.88$ & 655.66 & 204.17 & 62.24 & $2,355.05$ & 717.90 \\
\hline 351716116363701 & 14N/3E-24Q1S & 07/22/1997 & 2,189 & 667.28 & 166.05 & 50.62 & $2,355.05$ & 717.90 \\
\hline 351716116363701 & $14 \mathrm{~N} / 3 \mathrm{E}-24 \mathrm{Q} 1 \mathrm{~S}$ & 09/08/1997 & $2,190.06$ & 667.60 & 164.99 & 50.29 & $2,355.05$ & 717.90 \\
\hline 351716116363701 & 14N/3E-24Q1S & 07/08/1999 & $2,185.74$ & 666.29 & 169.31 & 51.61 & $2,355.05$ & 717.90 \\
\hline 351716116363701 & 14N/3E-24Q1S & $03 / 30 / 2000$ & $2,182.91$ & 665.42 & 172.14 & 52.47 & $2,355.05$ & 717.90 \\
\hline 351716116363701 & $14 \mathrm{~N} / 3 \mathrm{E}-24 \mathrm{Q} 1 \mathrm{~S}$ & $01 / 30 / 2002$ & $2,178.66$ & 664.13 & 176.39 & 53.77 & $2,355.05$ & 717.90 \\
\hline 351716116363701 & $14 \mathrm{~N} / 3 \mathrm{E}-24 \mathrm{Q} 1 \mathrm{~S}$ & $03 / 01 / 2005$ & $2,168.12$ & 660.92 & 186.93 & 56.98 & $2,355.05$ & 717.90 \\
\hline
\end{tabular}


Appendix 2. Water-level data for selected wells in Bicycle Basin, Fort Irwin National Training Center, California, 19552017.-Continued

[State well No.: See Well-Numbering System in text. Abbreviations: ID, identification number; mm/dd/yyyy, month/day/year; NAVD 88, North American Vertical Datum of $1988, \ldots$, well is dry]

\begin{tabular}{|c|c|c|c|c|c|c|c|c|}
\hline \multirow[t]{2}{*}{ Site ID } & \multirow{2}{*}{$\begin{array}{c}\text { State } \\
\text { well number }\end{array}$} & \multirow{2}{*}{$\begin{array}{c}\text { Date } \\
(\mathrm{mm} / \mathrm{dd} / \mathrm{yyyy})\end{array}$} & \multicolumn{2}{|c|}{$\begin{array}{c}\text { Water-surface altitude, } \\
\text { above NAVD } 88\end{array}$} & \multicolumn{2}{|c|}{$\begin{array}{l}\text { Depth to water, below } \\
\text { land surface }\end{array}$} & \multicolumn{2}{|c|}{$\begin{array}{c}\text { Land-surface altitude, } \\
\text { above NAVD } 88^{1}\end{array}$} \\
\hline & & & (feet) & (meters) & (feet) & (meters) & (feet) & (meters) \\
\hline 351716116363701 & $14 \mathrm{~N} / 3 \mathrm{E}-24 \mathrm{Q} 1 \mathrm{~S}$ & $11 / 06 / 2007$ & $2,163.35$ & 659.46 & 191.7 & 58.44 & $2,355.05$ & 717.90 \\
\hline 351716116363701 & $14 \mathrm{~N} / 3 \mathrm{E}-24 \mathrm{Q} 1 \mathrm{~S}$ & $12 / 19 / 2007$ & $2,162.98$ & 659.35 & 192.07 & 58.55 & $2,355.05$ & 717.90 \\
\hline 351716116363701 & $14 \mathrm{~N} / 3 \mathrm{E}-24 \mathrm{Q} 1 \mathrm{~S}$ & $07 / 28 / 2008$ & $2,162.33$ & 659.15 & 192.72 & 58.75 & $2,355.05$ & 717.90 \\
\hline 351716116363701 & 14N/3E-24Q1S & $01 / 22 / 2009$ & $2,161.57$ & 658.92 & 193.48 & 58.98 & $2,355.05$ & 717.90 \\
\hline 351716116363701 & 14N/3E-24Q1S & $01 / 26 / 2009$ & $2,161.45$ & 658.88 & 193.6 & 59.02 & $2,355.05$ & 717.90 \\
\hline 351716116363701 & 14N/3E-24Q1S & 04/08/2009 & $2,160.57$ & 658.61 & 194.48 & 59.28 & $2,355.05$ & 717.90 \\
\hline 351716116363701 & 14N/3E-24Q1S & $04 / 27 / 2011$ & $2,156.42$ & 657.35 & 198.63 & 60.55 & $2,355.05$ & 717.90 \\
\hline 351716116363701 & 14N/3E-24Q1S & $10 / 24 / 2011$ & $2,155.2$ & 656.98 & 199.85 & 60.92 & $2,355.05$ & 717.90 \\
\hline 351716116363701 & 14N/3E-24Q1S & $10 / 30 / 2011$ & $2,155.4$ & 657.04 & 199.65 & 60.86 & $2,355.05$ & 717.90 \\
\hline 351716116363701 & $14 \mathrm{~N} / 3 \mathrm{E}-24 \mathrm{Q} 1 \mathrm{~S}$ & $02 / 13 / 2012$ & $2,155.65$ & 657.11 & 199.4 & 60.78 & $2,355.05$ & 717.90 \\
\hline 351716116363701 & 14N/3E-24Q1S & $02 / 21 / 2012$ & $2,155.36$ & 657.03 & 199.69 & 60.87 & $2,355.05$ & 717.90 \\
\hline 351716116363701 & 14N/3E-24Q1S & $08 / 30 / 2012$ & $2,153.28$ & 656.39 & 201.77 & 61.51 & $2,355.05$ & 717.90 \\
\hline 351716116363701 & 14N/3E-24Q1S & $04 / 17 / 2013$ & $2,153.07$ & 656.33 & 201.98 & 61.57 & $2,355.05$ & 717.90 \\
\hline 351716116363701 & $14 \mathrm{~N} / 3 \mathrm{E}-24 \mathrm{Q} 1 \mathrm{~S}$ & 09/10/2013 & $2,154.13$ & 656.65 & 200.92 & 61.25 & $2,355.05$ & 717.90 \\
\hline 351716116363701 & 14N/3E-24Q1S & $08 / 01 / 2014$ & $2,152.54$ & 656.17 & 202.51 & 61.73 & $2,355.05$ & 717.90 \\
\hline 351716116363701 & $14 \mathrm{~N} / 3 \mathrm{E}-24 \mathrm{Q} 1 \mathrm{~S}$ & $12 / 22 / 2015$ & $2,151.05$ & 655.71 & 204 & 62.19 & $2,355.05$ & 717.90 \\
\hline 351716116363701 & $14 \mathrm{~N} / 3 \mathrm{E}-24 \mathrm{Q} 1 \mathrm{~S}$ & $04 / 05 / 2016$ & $2,150.69$ & 655.60 & 204.36 & 62.30 & $2,355.05$ & 717.90 \\
\hline 351716116363701 & 14N/3E-24Q1S & $05 / 24 / 2016$ & $2,150.15$ & 655.44 & 204.9 & 62.46 & $2,355.05$ & 717.90 \\
\hline 351716116363701 & $14 \mathrm{~N} / 3 \mathrm{E}-24 \mathrm{Q} 1 \mathrm{~S}$ & $06 / 06 / 2016$ & $2,150.17$ & 655.44 & 204.88 & 62.45 & $2,355.05$ & 717.90 \\
\hline 351716116363701 & $14 \mathrm{~N} / 3 \mathrm{E}-24 \mathrm{Q} 1 \mathrm{~S}$ & $12 / 09 / 2016$ & $2,148.3$ & 654.87 & 206.75 & 63.02 & $2,355.05$ & 717.90 \\
\hline 351716116363701 & 14N/3E-24Q1S & 03/07/2017 & $2,149.03$ & 655.10 & 206.02 & 62.80 & $2,355.05$ & 717.90 \\
\hline 351716116363701 & $14 \mathrm{~N} / 3 \mathrm{E}-24 \mathrm{Q} 1 \mathrm{~S}$ & $05 / 16 / 2017$ & $2,149.54$ & 655.25 & 205.51 & 62.65 & $2,355.05$ & 717.90 \\
\hline \multicolumn{9}{|c|}{ Local well ID: BLA3-2 } \\
\hline 351716116363702 & $14 \mathrm{~N} / 3 \mathrm{E}-24 \mathrm{Q} 2 \mathrm{~S}$ & $07 / 14 / 1997$ & $2,152.84$ & 656.26 & 202.21 & 61.64 & $2,355.05$ & 717.90 \\
\hline 351716116363702 & $14 \mathrm{~N} / 3 \mathrm{E}-24 \mathrm{Q} 2 \mathrm{~S}$ & 07/23/1997 & $2,189.59$ & 667.46 & 165.46 & 50.44 & $2,355.05$ & 717.90 \\
\hline 351716116363702 & $14 \mathrm{~N} / 3 \mathrm{E}-24 \mathrm{Q} 2 \mathrm{~S}$ & 09/08/1997 & $2,189.76$ & 667.51 & 165.29 & 50.39 & $2,355.05$ & 717.90 \\
\hline 351716116363702 & $14 \mathrm{~N} / 3 \mathrm{E}-24 \mathrm{Q} 2 \mathrm{~S}$ & 07/08/1999 & $2,185.06$ & 666.08 & 169.99 & 51.82 & $2,355.05$ & 717.90 \\
\hline 351716116363702 & $14 \mathrm{~N} / 3 \mathrm{E}-24 \mathrm{Q} 2 \mathrm{~S}$ & $03 / 30 / 2000$ & $2,181.25$ & 664.92 & 173.8 & 52.98 & $2,355.05$ & 717.90 \\
\hline 351716116363702 & $14 \mathrm{~N} / 3 \mathrm{E}-24 \mathrm{Q} 2 \mathrm{~S}$ & $01 / 30 / 2002$ & $2,177.75$ & 663.85 & 177.3 & 54.05 & $2,355.05$ & 717.90 \\
\hline
\end{tabular}


Appendix 2. Water-level data for selected wells in Bicycle Basin, Fort Irwin National Training Center, California, 19552017.-Continued

[State well No.: See Well-Numbering System in text. Abbreviations: ID, identification number; mm/dd/yyyy, month/day/year; NAVD 88, North American Vertical Datum of $1988, \ldots$, well is dry]

\begin{tabular}{|c|c|c|c|c|c|c|c|c|}
\hline \multirow[t]{2}{*}{ Site ID } & \multirow{2}{*}{$\begin{array}{c}\text { State } \\
\text { well number }\end{array}$} & \multirow{2}{*}{$\begin{array}{c}\text { Date } \\
\text { (mm/dd/yyyy) }\end{array}$} & \multicolumn{2}{|c|}{$\begin{array}{c}\text { Water-surface altitude, } \\
\text { above NAVD } 88\end{array}$} & \multicolumn{2}{|c|}{$\begin{array}{l}\text { Depth to water, below } \\
\text { land surface }\end{array}$} & \multicolumn{2}{|c|}{$\begin{array}{l}\text { Land-surface altitude, } \\
\text { above NAVD } 88^{1}\end{array}$} \\
\hline & & & (feet) & (meters) & (feet) & (meters) & (feet) & (meters) \\
\hline 351716116363702 & 14N/3E-24Q2S & $03 / 01 / 2005$ & $2,167.48$ & 660.72 & 187.57 & 57.18 & $2,355.05$ & 717.90 \\
\hline 351716116363702 & $14 \mathrm{~N} / 3 \mathrm{E}-24 \mathrm{Q} 2 \mathrm{~S}$ & $11 / 06 / 2007$ & $2,162.2$ & 659.11 & 192.85 & 58.79 & $2,355.05$ & 717.90 \\
\hline 351716116363702 & $14 \mathrm{~N} / 3 \mathrm{E}-24 \mathrm{Q} 2 \mathrm{~S}$ & $04 / 30 / 2008$ & $2,162.93$ & 659.33 & 192.12 & 58.56 & $2,355.05$ & 717.90 \\
\hline 351716116363702 & $14 \mathrm{~N} / 3 \mathrm{E}-24 \mathrm{Q} 2 \mathrm{~S}$ & $07 / 28 / 2008$ & $2,161.27$ & 658.83 & 193.78 & 59.07 & $2,355.05$ & 717.90 \\
\hline 351716116363702 & $14 \mathrm{~N} / 3 \mathrm{E}-24 \mathrm{Q} 2 \mathrm{~S}$ & $01 / 22 / 2009$ & $2,160.74$ & 658.67 & 194.31 & 59.23 & $2,355.05$ & 717.90 \\
\hline 351716116363702 & 14N/3E-24Q2S & $01 / 26 / 2009$ & $2,160.58$ & 658.62 & 194.47 & 59.28 & $2,355.05$ & 717.90 \\
\hline 351716116363702 & $14 \mathrm{~N} / 3 \mathrm{E}-24 \mathrm{Q} 2 \mathrm{~S}$ & $12 / 17 / 2010$ & $2,156.59$ & 657.40 & 198.46 & 60.50 & $2,355.05$ & 717.90 \\
\hline 351716116363702 & $14 \mathrm{~N} / 3 \mathrm{E}-24 \mathrm{Q} 2 \mathrm{~S}$ & $04 / 27 / 2011$ & $2,155.58$ & 657.09 & 199.47 & 60.81 & $2,355.05$ & 717.90 \\
\hline 351716116363702 & $14 \mathrm{~N} / 3 \mathrm{E}-24 \mathrm{Q} 2 \mathrm{~S}$ & $10 / 24 / 2011$ & $2,154.41$ & 656.74 & 200.64 & 61.16 & $2,355.05$ & 717.90 \\
\hline 351716116363702 & $14 \mathrm{~N} / 3 \mathrm{E}-24 \mathrm{Q} 2 \mathrm{~S}$ & $10 / 30 / 2011$ & $2,154.6$ & 656.79 & 200.45 & 61.10 & $2,355.05$ & 717.90 \\
\hline 351716116363702 & $14 \mathrm{~N} / 3 \mathrm{E}-24 \mathrm{Q} 2 \mathrm{~S}$ & $02 / 13 / 2012$ & $2,154.98$ & 656.91 & 200.07 & 60.99 & $2,355.05$ & 717.90 \\
\hline 351716116363702 & $14 \mathrm{~N} / 3 \mathrm{E}-24 \mathrm{Q} 2 \mathrm{~S}$ & $02 / 21 / 2012$ & $2,154.6$ & 656.79 & 200.45 & 61.10 & $2,355.05$ & 717.90 \\
\hline 351716116363702 & 14N/3E-24Q2S & 08/30/2012 & $2,152.15$ & 656.05 & 202.9 & 61.85 & $2,355.05$ & 717.90 \\
\hline 351716116363702 & $14 \mathrm{~N} / 3 \mathrm{E}-24 \mathrm{Q} 2 \mathrm{~S}$ & $04 / 17 / 2013$ & $2,152.55$ & 656.17 & 202.5 & 61.73 & $2,355.05$ & 717.90 \\
\hline 351716116363702 & $14 \mathrm{~N} / 3 \mathrm{E}-24 \mathrm{Q} 2 \mathrm{~S}$ & 09/10/2013 & $2,153.77$ & 656.54 & 201.28 & 61.36 & $2,355.05$ & 717.90 \\
\hline 351716116363702 & $14 \mathrm{~N} / 3 \mathrm{E}-24 \mathrm{Q} 2 \mathrm{~S}$ & $12 / 22 / 2015$ & $2,150.47$ & 655.54 & 204.58 & 62.36 & $2,355.05$ & 717.90 \\
\hline 351716116363702 & 14N/3E-24Q2S & $12 / 22 / 2015$ & $2,150.52$ & 655.55 & 204.53 & 62.35 & $2,355.05$ & 717.90 \\
\hline 351716116363702 & $14 \mathrm{~N} / 3 \mathrm{E}-24 \mathrm{Q} 2 \mathrm{~S}$ & $12 / 22 / 2015$ & $2,150.52$ & 655.55 & 204.53 & 62.35 & $2,355.05$ & 717.90 \\
\hline 351716116363702 & 14N/3E-24Q2S & $04 / 05 / 2016$ & $2,150.11$ & 655.43 & 204.94 & 62.47 & $2,355.05$ & 717.90 \\
\hline 351716116363702 & 14N/3E-24Q2S & $05 / 24 / 2016$ & $2,149.36$ & 655.20 & 205.69 & 62.70 & $2,355.05$ & 717.90 \\
\hline 351716116363702 & $14 \mathrm{~N} / 3 \mathrm{E}-24 \mathrm{Q} 2 \mathrm{~S}$ & $06 / 06 / 2016$ & $2,149.36$ & 655.20 & 205.69 & 62.70 & $2,355.05$ & 717.90 \\
\hline 351716116363702 & $14 \mathrm{~N} / 3 \mathrm{E}-24 \mathrm{Q} 2 \mathrm{~S}$ & $12 / 09 / 2016$ & $2,147.69$ & 654.69 & 207.36 & 63.21 & $2,355.05$ & 717.90 \\
\hline 351716116363702 & $14 \mathrm{~N} / 3 \mathrm{E}-24 \mathrm{Q} 2 \mathrm{~S}$ & $12 / 09 / 2016$ & $2,147.51$ & 654.63 & 207.54 & 63.27 & $2,355.05$ & 717.90 \\
\hline 351716116363702 & $14 \mathrm{~N} / 3 \mathrm{E}-24 \mathrm{Q} 2 \mathrm{~S}$ & $03 / 07 / 2017$ & $2,148.58$ & 654.96 & 206.47 & 62.94 & $2,355.05$ & 717.90 \\
\hline 351716116363702 & 14N/3E-24Q2S & $05 / 16 / 2017$ & $2,149.37$ & 655.20 & 205.68 & 62.70 & $2,355.05$ & 717.90 \\
\hline \multicolumn{9}{|c|}{ Local well ID: BLA3-3 } \\
\hline 351716116363703 & $14 \mathrm{~N} / 3 \mathrm{E}-24 \mathrm{Q} 3 \mathrm{~S}$ & 07/14/1997 & $2,164.59$ & 659.84 & 190.46 & 58.06 & $2,355.05$ & 717.90 \\
\hline 351716116363703 & $14 \mathrm{~N} / 3 \mathrm{E}-24 \mathrm{Q} 3 \mathrm{~S}$ & 07/23/1997 & $2,189.15$ & 667.33 & 165.9 & 50.57 & $2,355.05$ & 717.90 \\
\hline 351716116363703 & $14 \mathrm{~N} / 3 \mathrm{E}-24 \mathrm{Q} 3 \mathrm{~S}$ & 09/08/1997 & $2,188.55$ & 667.14 & 166.5 & 50.75 & $2,355.05$ & 717.90 \\
\hline
\end{tabular}


Appendix 2. Water-level data for selected wells in Bicycle Basin, Fort Irwin National Training Center, California, 19552017.-Continued

[State well No.: See Well-Numbering System in text. Abbreviations: ID, identification number; mm/dd/yyyy, month/day/year; NAVD 88, North American Vertical Datum of $1988, \ldots$, well is dry]

\begin{tabular}{|c|c|c|c|c|c|c|c|c|}
\hline \multirow[t]{2}{*}{ Site ID } & \multirow{2}{*}{$\begin{array}{c}\text { State } \\
\text { well number }\end{array}$} & \multirow{2}{*}{$\begin{array}{c}\text { Date } \\
\text { (mm/dd/yyyy) }\end{array}$} & \multicolumn{2}{|c|}{$\begin{array}{c}\text { Water-surface altitude, } \\
\text { above NAVD } 88\end{array}$} & \multicolumn{2}{|c|}{$\begin{array}{l}\text { Depth to water, below } \\
\text { land surface }\end{array}$} & \multicolumn{2}{|c|}{$\begin{array}{c}\text { Land-surface altitude, } \\
\text { above NAVD } 88^{1}\end{array}$} \\
\hline & & & (feet) & (meters) & (feet) & (meters) & (feet) & (meters) \\
\hline 351716116363703 & 14N/3E-24Q3S & $07 / 08 / 1999$ & $2,183.95$ & 665.74 & 171.1 & 52.16 & $2,355.05$ & 717.90 \\
\hline 351716116363703 & 14N/3E-24Q3S & $03 / 30 / 2000$ & $2,180.68$ & 664.74 & 174.37 & 53.15 & $2,355.05$ & 717.90 \\
\hline 351716116363703 & 14N/3E-24Q3S & $11 / 06 / 2007$ & $2,160.31$ & 658.53 & 194.74 & 59.36 & $2,355.05$ & 717.90 \\
\hline 351716116363703 & 14N/3E-24Q3S & $12 / 19 / 2007$ & $2,160.3$ & 658.53 & 194.75 & 59.37 & $2,355.05$ & 717.90 \\
\hline 351716116363703 & $14 N / 3 E-24 Q 3 S$ & $02 / 14 / 2008$ & $2,160.84$ & 658.70 & 194.21 & 59.20 & $2,355.05$ & 717.90 \\
\hline 351716116363703 & 14N/3E-24Q3S & $04 / 30 / 2008$ & $2,161.52$ & 658.90 & 193.53 & 58.99 & $2,355.05$ & 717.90 \\
\hline 351716116363703 & $14 \mathrm{~N} / 3 \mathrm{E}-24 \mathrm{Q} 3 \mathrm{~S}$ & $04 / 08 / 2009$ & $2,157.85$ & 657.78 & 197.2 & 60.11 & $2,355.05$ & 717.90 \\
\hline 351716116363703 & 14N/3E-24Q3S & $10 / 07 / 2009$ & $2,156.4$ & 657.34 & 198.65 & 60.56 & $2,355.05$ & 717.90 \\
\hline 351716116363703 & 14N/3E-24Q3S & $06 / 14 / 2010$ & $2,156.82$ & 657.47 & 198.23 & 60.43 & $2,355.05$ & 717.90 \\
\hline 351716116363703 & $14 N / 3 E-24 Q 3 S$ & $12 / 17 / 2010$ & $2,154.85$ & 656.87 & 200.2 & 61.03 & $2,355.05$ & 717.90 \\
\hline 351716116363703 & 14N/3E-24Q3S & $04 / 27 / 2011$ & $2,153.63$ & 656.50 & 201.42 & 61.40 & $2,355.05$ & 717.90 \\
\hline 351716116363703 & 14N/3E-24Q3S & $10 / 24 / 2011$ & $2,152.76$ & 656.23 & 202.29 & 61.66 & $2,355.05$ & 717.90 \\
\hline 351716116363703 & 14N/3E-24Q3S & $10 / 30 / 2011$ & $2,152.94$ & 656.29 & 202.11 & 61.61 & $2,355.05$ & 717.90 \\
\hline 351716116363703 & 14N/3E-24Q3S & $02 / 13 / 2012$ & $2,153.45$ & 656.44 & 201.6 & 61.45 & $2,355.05$ & 717.90 \\
\hline 351716116363703 & $14 N / 3 E-24 Q 3 S$ & $02 / 21 / 2012$ & $2,153.08$ & 656.33 & 201.97 & 61.57 & $2,355.05$ & 717.90 \\
\hline 351716116363703 & 14N/3E-24Q3S & $02 / 09 / 2015$ & $2,150.18$ & 655.45 & 204.87 & 62.45 & $2,355.05$ & 717.90 \\
\hline 351716116363703 & $14 \mathrm{~N} / 3 \mathrm{E}-24 \mathrm{Q} 3 \mathrm{~S}$ & $02 / 16 / 2015$ & $2,150.05$ & 655.41 & 205 & 62.49 & $2,355.05$ & 717.90 \\
\hline 351716116363703 & $14 N / 3 E-24 Q 3 S$ & $02 / 16 / 2015$ & $2,150.1$ & 655.42 & 204.95 & 62.48 & $2,355.05$ & 717.90 \\
\hline 351716116363703 & 14N/3E-24Q3S & $05 / 20 / 2015$ & $2,149.76$ & 655.32 & 205.29 & 62.58 & $2,355.05$ & 717.90 \\
\hline 351716116363703 & 14N/3E-24Q3S & $05 / 24 / 2016$ & $2,147.81$ & 654.72 & 207.24 & 63.17 & $2,355.05$ & 717.90 \\
\hline 351716116363703 & $14 N / 3 E-24 Q 3 S$ & $06 / 06 / 2016$ & $2,147.79$ & 654.72 & 207.26 & 63.18 & $2,355.05$ & 717.90 \\
\hline 351716116363703 & $14 N / 3 E-24 Q 3 S$ & $12 / 09 / 2016$ & $2,146.11$ & 654.21 & 208.94 & 63.69 & $2,355.05$ & 717.90 \\
\hline 351716116363703 & $14 N / 3 E-24 Q 3 S$ & 03/07/2017 & $2,147.35$ & 654.58 & 207.7 & 63.31 & $2,355.05$ & 717.90 \\
\hline 351716116363703 & 14N/3E-24Q3S & $05 / 16 / 2017$ & $2,148.11$ & 654.82 & 206.94 & 63.08 & $2,355.05$ & 717.90 \\
\hline \multicolumn{9}{|c|}{ Local well ID: BLA3-4 } \\
\hline 351716116363704 & 14N/3E-24Q4S & $07 / 14 / 1997$ & $2,175.16$ & 663.06 & 179.89 & 54.84 & $2,355.05$ & 717.90 \\
\hline 351716116363704 & $14 N / 3 E-24 Q 4 S$ & $07 / 24 / 1997$ & $2,188.5$ & 667.13 & 166.55 & 50.77 & $2,355.05$ & 717.90 \\
\hline 351716116363704 & $14 N / 3 E-24 Q 4 S$ & 09/08/1997 & $2,187.96$ & 666.96 & 167.09 & 50.93 & $2,355.05$ & 717.90 \\
\hline 351716116363704 & 14N/3E-24Q4S & 07/08/1999 & $2,183.11$ & 665.48 & 171.94 & 52.41 & $2,355.05$ & 717.90 \\
\hline
\end{tabular}


Appendix 2. Water-level data for selected wells in Bicycle Basin, Fort Irwin National Training Center, California, 19552017.-Continued

[State well No.: See Well-Numbering System in text. Abbreviations: ID, identification number; mm/dd/yyyy, month/day/year; NAVD 88, North American Vertical Datum of $1988, \ldots$, well is dry]

\begin{tabular}{|c|c|c|c|c|c|c|c|c|}
\hline \multirow[t]{2}{*}{ Site ID } & \multirow{2}{*}{$\begin{array}{c}\text { State } \\
\text { well number }\end{array}$} & \multirow{2}{*}{$\begin{array}{c}\text { Date } \\
\text { (mm/dd/yyyy) }\end{array}$} & \multicolumn{2}{|c|}{$\begin{array}{c}\text { Water-surface altitude, } \\
\text { above NAVD } 88\end{array}$} & \multicolumn{2}{|c|}{$\begin{array}{l}\text { Depth to water, below } \\
\text { land surface }\end{array}$} & \multicolumn{2}{|c|}{$\begin{array}{l}\text { Land-surface altitude, } \\
\text { above NAVD } 88^{1}\end{array}$} \\
\hline & & & (feet) & (meters) & (feet) & (meters) & (feet) & (meters) \\
\hline 351716116363704 & $14 \mathrm{~N} / 3 \mathrm{E}-24 \mathrm{Q} 4 \mathrm{~S}$ & $03 / 30 / 2000$ & $2,179.73$ & 664.45 & 175.32 & 53.44 & $2,355.05$ & 717.90 \\
\hline 351716116363704 & $14 \mathrm{~N} / 3 \mathrm{E}-24 \mathrm{Q} 4 \mathrm{~S}$ & $01 / 30 / 2002$ & $2,175.22$ & 663.08 & 179.83 & 54.82 & $2,355.05$ & 717.90 \\
\hline 351716116363704 & $14 \mathrm{~N} / 3 \mathrm{E}-24 \mathrm{Q} 4 \mathrm{~S}$ & $12 / 19 / 2007$ & $2,159.15$ & 658.18 & 195.9 & 59.72 & $2,355.05$ & 717.90 \\
\hline 351716116363704 & $14 \mathrm{~N} / 3 \mathrm{E}-24 \mathrm{Q} 4 \mathrm{~S}$ & $02 / 14 / 2008$ & $2,159.8$ & 658.38 & 195.25 & 59.52 & $2,355.05$ & 717.90 \\
\hline 351716116363704 & $14 \mathrm{~N} / 3 \mathrm{E}-24 \mathrm{Q} 4 \mathrm{~S}$ & $04 / 30 / 2008$ & $2,160.5$ & 658.59 & 194.55 & 59.31 & $2,355.05$ & 717.90 \\
\hline 351716116363704 & $14 \mathrm{~N} / 3 \mathrm{E}-24 \mathrm{Q} 4 \mathrm{~S}$ & $07 / 28 / 2008$ & $2,158.44$ & 657.96 & 196.61 & 59.93 & $2,355.05$ & 717.90 \\
\hline 351716116363704 & $14 \mathrm{~N} / 3 \mathrm{E}-24 \mathrm{Q} 4 \mathrm{~S}$ & $10 / 07 / 2009$ & $2,155.23$ & 656.99 & 199.82 & 60.91 & $2,355.05$ & 717.90 \\
\hline 351716116363704 & $14 \mathrm{~N} / 3 \mathrm{E}-24 \mathrm{Q} 4 \mathrm{~S}$ & $06 / 14 / 2010$ & $2,155.95$ & 657.21 & 199.1 & 60.69 & $2,355.05$ & 717.90 \\
\hline 351716116363704 & $14 \mathrm{~N} / 3 \mathrm{E}-24 \mathrm{Q} 4 \mathrm{~S}$ & $12 / 17 / 2010$ & $2,153.75$ & 656.54 & 201.3 & 61.36 & $2,355.05$ & 717.90 \\
\hline 351716116363704 & $14 \mathrm{~N} / 3 \mathrm{E}-24 \mathrm{Q} 4 \mathrm{~S}$ & $04 / 27 / 2011$ & $2,152.56$ & 656.17 & 202.49 & 61.73 & $2,355.05$ & 717.90 \\
\hline 351716116363704 & $14 \mathrm{~N} / 3 \mathrm{E}-24 \mathrm{Q} 4 \mathrm{~S}$ & $10 / 24 / 2011$ & $2,151.67$ & 655.90 & 203.38 & 62.00 & $2,355.05$ & 717.90 \\
\hline 351716116363704 & $14 \mathrm{~N} / 3 \mathrm{E}-24 \mathrm{Q} 4 \mathrm{~S}$ & $10 / 25 / 2011$ & $2,151.81$ & 655.94 & 203.24 & 61.95 & $2,355.05$ & 717.90 \\
\hline 351716116363704 & $14 \mathrm{~N} / 3 \mathrm{E}-24 \mathrm{Q} 4 \mathrm{~S}$ & $02 / 13 / 2012$ & $2,152.41$ & 656.13 & 202.64 & 61.77 & $2,355.05$ & 717.90 \\
\hline 351716116363704 & $14 \mathrm{~N} / 3 \mathrm{E}-24 \mathrm{Q} 4 \mathrm{~S}$ & $02 / 21 / 2012$ & $2,152.09$ & 656.03 & 202.96 & 61.87 & $2,355.05$ & 717.90 \\
\hline 351716116363704 & $14 \mathrm{~N} / 3 \mathrm{E}-24 \mathrm{Q} 4 \mathrm{~S}$ & 08/30/2012 & $2,149.25$ & 655.16 & 205.8 & 62.73 & $2,355.05$ & 717.90 \\
\hline 351716116363704 & $14 \mathrm{~N} / 3 \mathrm{E}-24 \mathrm{Q} 4 \mathrm{~S}$ & $02 / 16 / 2015$ & $2,149.23$ & 655.16 & 205.82 & 62.74 & $2,355.05$ & 717.90 \\
\hline 351716116363704 & $14 \mathrm{~N} / 3 \mathrm{E}-24 \mathrm{Q} 4 \mathrm{~S}$ & $05 / 20 / 2015$ & $2,148.89$ & 655.05 & 206.16 & 62.84 & $2,355.05$ & 717.90 \\
\hline 351716116363704 & $14 \mathrm{~N} / 3 \mathrm{E}-24 \mathrm{Q} 4 \mathrm{~S}$ & $12 / 22 / 2015$ & $2,148.36$ & 654.89 & 206.69 & 63.01 & $2,355.05$ & 717.90 \\
\hline 351716116363704 & $14 \mathrm{~N} / 3 \mathrm{E}-24 \mathrm{Q} 4 \mathrm{~S}$ & $05 / 24 / 2016$ & $2,146.9$ & 654.45 & 208.15 & 63.45 & $2,355.05$ & 717.90 \\
\hline 351716116363704 & $14 \mathrm{~N} / 3 \mathrm{E}-24 \mathrm{Q} 4 \mathrm{~S}$ & 06/06/2016 & $2,146.87$ & 654.44 & 208.18 & 63.46 & $2,355.05$ & 717.90 \\
\hline 351716116363704 & $14 \mathrm{~N} / 3 \mathrm{E}-24 \mathrm{Q} 4 \mathrm{~S}$ & $12 / 09 / 2016$ & $2,145.17$ & 653.92 & 209.88 & 63.98 & $2,355.05$ & 717.90 \\
\hline 351716116363704 & $14 \mathrm{~N} / 3 \mathrm{E}-24 \mathrm{Q} 4 \mathrm{~S}$ & 03/07/2017 & $2,146.61$ & 654.36 & 208.44 & 63.54 & $2,355.05$ & 717.90 \\
\hline 351716116363704 & $14 \mathrm{~N} / 3 \mathrm{E}-24 \mathrm{Q} 4 \mathrm{~S}$ & $05 / 16 / 2017$ & $2,147.43$ & 654.61 & 207.62 & 63.29 & $2,355.05$ & 717.90 \\
\hline \multicolumn{9}{|c|}{ Local well ID: BLA3-5 } \\
\hline 351716116363705 & $14 \mathrm{~N} / 3 \mathrm{E}-24 \mathrm{Q} 5 \mathrm{~S}$ & 07/14/1997 & 2,184 & 665.76 & 171.05 & 52.14 & $2,355.05$ & 717.90 \\
\hline 351716116363705 & $14 \mathrm{~N} / 3 \mathrm{E}-24 \mathrm{Q} 5 \mathrm{~S}$ & 07/24/1997 & $2,188.79$ & 667.22 & 166.26 & 50.68 & $2,355.05$ & 717.90 \\
\hline 351716116363705 & $14 \mathrm{~N} / 3 \mathrm{E}-24 \mathrm{Q} 5 \mathrm{~S}$ & 09/08/1997 & $2,188.31$ & 667.07 & 166.74 & 50.83 & $2,355.05$ & 717.90 \\
\hline 351716116363705 & $14 \mathrm{~N} / 3 \mathrm{E}-24 \mathrm{Q} 5 \mathrm{~S}$ & 07/08/1999 & $2,183.74$ & 665.68 & 171.31 & 52.22 & $2,355.05$ & 717.90 \\
\hline 351716116363705 & 14N/3E-24Q5S & $03 / 30 / 2000$ & $2,180.41$ & 664.66 & 174.64 & 53.24 & $2,355.05$ & 717.90 \\
\hline
\end{tabular}


Appendix 2. Water-level data for selected wells in Bicycle Basin, Fort Irwin National Training Center, California, 19552017.-Continued

[State well No.: See Well-Numbering System in text. Abbreviations: ID, identification number; mm/dd/yyyy, month/day/year; NAVD 88, North American Vertical Datum of $1988, \ldots$, well is dry]

\begin{tabular}{|c|c|c|c|c|c|c|c|c|}
\hline \multirow[t]{2}{*}{ Site ID } & \multirow{2}{*}{$\begin{array}{c}\text { State } \\
\text { well number }\end{array}$} & \multirow{2}{*}{$\begin{array}{c}\text { Date } \\
\text { (mm/dd/yyyy) }\end{array}$} & \multicolumn{2}{|c|}{$\begin{array}{c}\text { Water-surface altitude, } \\
\text { above NAVD } 88\end{array}$} & \multicolumn{2}{|c|}{$\begin{array}{l}\text { Depth to water, below } \\
\text { land surface }\end{array}$} & \multicolumn{2}{|c|}{$\begin{array}{c}\text { Land-surface altitude, } \\
\text { above NAVD } 88^{1}\end{array}$} \\
\hline & & & (feet) & (meters) & (feet) & (meters) & (feet) & (meters) \\
\hline 351716116363705 & $14 \mathrm{~N} / 3 \mathrm{E}-24 \mathrm{Q} 5 \mathrm{~S}$ & $01 / 30 / 2002$ & $2,175.91$ & 663.29 & 179.14 & 54.61 & $2,355.05$ & 717.90 \\
\hline 351716116363705 & $14 \mathrm{~N} / 3 \mathrm{E}-24 \mathrm{Q} 5 \mathrm{~S}$ & $03 / 01 / 2005$ & $2,165.32$ & 660.06 & 189.73 & 57.84 & $2,355.05$ & 717.90 \\
\hline 351716116363705 & $14 \mathrm{~N} / 3 \mathrm{E}-24 \mathrm{Q} 5 \mathrm{~S}$ & $02 / 14 / 2008$ & $2,160.33$ & 658.54 & 194.72 & 59.36 & $2,355.05$ & 717.90 \\
\hline 351716116363705 & 14N/3E-24Q5S & $04 / 30 / 2008$ & $2,160.92$ & 658.72 & 194.13 & 59.18 & $2,355.05$ & 717.90 \\
\hline 351716116363705 & 14N/3E-24Q5S & $07 / 28 / 2008$ & $2,159.29$ & 658.22 & 195.76 & 59.67 & $2,355.05$ & 717.90 \\
\hline 351716116363705 & $14 \mathrm{~N} / 3 \mathrm{E}-24 \mathrm{Q} 5 \mathrm{~S}$ & $01 / 22 / 2009$ & $2,158.74$ & 658.06 & 196.31 & 59.84 & $2,355.05$ & 717.90 \\
\hline 351716116363705 & 14N/3E-24Q5S & $06 / 14 / 2010$ & $2,156.64$ & 657.42 & 198.41 & 60.48 & $2,355.05$ & 717.90 \\
\hline 351716116363705 & $14 \mathrm{~N} / 3 \mathrm{E}-24 \mathrm{Q} 5 \mathrm{~S}$ & $12 / 17 / 2010$ & $2,154.51$ & 656.77 & 200.54 & 61.13 & $2,355.05$ & 717.90 \\
\hline 351716116363705 & $14 \mathrm{~N} / 3 \mathrm{E}-24 \mathrm{Q} 5 \mathrm{~S}$ & $04 / 27 / 2011$ & $2,153.37$ & 656.42 & 201.68 & 61.48 & $2,355.05$ & 717.90 \\
\hline 351716116363705 & $14 \mathrm{~N} / 3 \mathrm{E}-24 \mathrm{Q} 5 \mathrm{~S}$ & $10 / 24 / 2011$ & $2,152.22$ & 656.07 & 202.83 & 61.83 & $2,355.05$ & 717.90 \\
\hline 351716116363705 & $14 \mathrm{~N} / 3 \mathrm{E}-24 \mathrm{Q} 5 \mathrm{~S}$ & $10 / 30 / 2011$ & $2,152.36$ & 656.11 & 202.69 & 61.79 & $2,355.05$ & 717.90 \\
\hline 351716116363705 & 14N/3E-24Q5S & $02 / 13 / 2012$ & $2,152.91$ & 656.28 & 202.14 & 61.62 & $2,355.05$ & 717.90 \\
\hline 351716116363705 & $14 \mathrm{~N} / 3 \mathrm{E}-24 \mathrm{Q} 5 \mathrm{~S}$ & $02 / 21 / 2012$ & $2,152.68$ & 656.21 & 202.37 & 61.69 & $2,355.05$ & 717.90 \\
\hline 351716116363705 & $14 \mathrm{~N} / 3 \mathrm{E}-24 \mathrm{Q} 5 \mathrm{~S}$ & $08 / 30 / 2012$ & $2,150.11$ & 655.43 & 204.94 & 62.47 & $2,355.05$ & 717.90 \\
\hline 351716116363705 & $14 \mathrm{~N} / 3 \mathrm{E}-24 \mathrm{Q} 5 \mathrm{~S}$ & $04 / 17 / 2013$ & $2,150.58$ & 655.57 & 204.47 & 62.33 & $2,355.05$ & 717.90 \\
\hline 351716116363705 & $14 \mathrm{~N} / 3 \mathrm{E}-24 \mathrm{Q} 5 \mathrm{~S}$ & $05 / 20 / 2015$ & $2,149.36$ & 655.20 & 205.69 & 62.70 & $2,355.05$ & 717.90 \\
\hline 351716116363705 & $14 \mathrm{~N} / 3 \mathrm{E}-24 \mathrm{Q} 5 \mathrm{~S}$ & $12 / 22 / 2015$ & $2,148.71$ & 655.00 & 206.34 & 62.90 & $2,355.05$ & 717.90 \\
\hline 351716116363705 & $14 \mathrm{~N} / 3 \mathrm{E}-24 \mathrm{Q} 5 \mathrm{~S}$ & $02 / 17 / 2016$ & $2,149.07$ & 655.11 & 205.98 & 62.79 & $2,355.05$ & 717.90 \\
\hline 351716116363705 & $14 \mathrm{~N} / 3 \mathrm{E}-24 \mathrm{Q} 5 \mathrm{~S}$ & $05 / 24 / 2016$ & $2,147.59$ & 654.66 & 207.46 & 63.24 & $2,355.05$ & 717.90 \\
\hline 351716116363705 & $14 \mathrm{~N} / 3 \mathrm{E}-24 \mathrm{Q} 5 \mathrm{~S}$ & $06 / 06 / 2016$ & $2,147.56$ & 654.65 & 207.49 & 63.25 & $2,355.05$ & 717.90 \\
\hline 351716116363705 & 14N/3E-24Q5S & $12 / 09 / 2016$ & $2,145.64$ & 654.06 & 209.41 & 63.84 & $2,355.05$ & 717.90 \\
\hline 351716116363705 & $14 \mathrm{~N} / 3 \mathrm{E}-24 \mathrm{Q} 5 \mathrm{~S}$ & $12 / 09 / 2016$ & $2,145.73$ & 654.09 & 209.32 & 63.81 & $2,355.05$ & 717.90 \\
\hline 351716116363705 & $14 \mathrm{~N} / 3 \mathrm{E}-24 \mathrm{Q} 5 \mathrm{~S}$ & 03/07/2017 & $2,146.92$ & 654.45 & 208.13 & 63.44 & $2,355.05$ & 717.90 \\
\hline 351716116363705 & 14N/3E-24Q5S & $05 / 16 / 2017$ & $2,147.7$ & 654.69 & 207.35 & 63.21 & $2,355.05$ & 717.90 \\
\hline \multicolumn{9}{|c|}{ Local well ID: BLA5-1 } \\
\hline 351638116374301 & $14 \mathrm{~N} / 3 \mathrm{E}-26 \mathrm{~K} 1 \mathrm{~S}$ & $04 / 25 / 2011$ & 2,160 & 658.44 & 185.15 & 56.44 & 2,345 & 714.83 \\
\hline 351638116374301 & $14 \mathrm{~N} / 3 \mathrm{E}-26 \mathrm{~K} 1 \mathrm{~S}$ & $10 / 25 / 2011$ & 2,159 & 658.14 & 186.08 & 56.72 & 2,345 & 714.83 \\
\hline 351638116374301 & $14 \mathrm{~N} / 3 \mathrm{E}-26 \mathrm{~K} 1 \mathrm{~S}$ & $10 / 31 / 2011$ & 2,159 & 658.14 & 186.24 & 56.77 & 2,345 & 714.83 \\
\hline 351638116374301 & $14 \mathrm{~N} / 3 \mathrm{E}-26 \mathrm{~K} 1 \mathrm{~S}$ & $02 / 13 / 2012$ & 2,158 & 657.83 & 186.54 & 56.86 & 2,345 & 714.83 \\
\hline
\end{tabular}


Appendix 2. Water-level data for selected wells in Bicycle Basin, Fort Irwin National Training Center, California, 19552017.-Continued

[State well No.: See Well-Numbering System in text. Abbreviations: ID, identification number; mm/dd/yyyy, month/day/year; NAVD 88, North American Vertical Datum of 1988, -, well is dry]

\begin{tabular}{|c|c|c|c|c|c|c|c|c|}
\hline \multirow[t]{2}{*}{ Site ID } & \multirow{2}{*}{$\begin{array}{c}\text { State } \\
\text { well number }\end{array}$} & \multirow{2}{*}{$\begin{array}{c}\text { Date } \\
\text { (mm/dd/yyyy) }\end{array}$} & \multicolumn{2}{|c|}{$\begin{array}{c}\text { Water-surface altitude, } \\
\text { above NAVD } 88\end{array}$} & \multicolumn{2}{|c|}{$\begin{array}{c}\text { Depth to water, below } \\
\text { land surface }\end{array}$} & \multicolumn{2}{|c|}{$\begin{array}{c}\text { Land-surface altitude, } \\
\text { above NAVD } 88^{1}\end{array}$} \\
\hline & & & (feet) & (meters) & (feet) & (meters) & (feet) & (meters) \\
\hline 351638116374301 & $14 \mathrm{~N} / 3 \mathrm{E}-26 \mathrm{~K} 1 \mathrm{~S}$ & $03 / 27 / 2012$ & 2,158 & 657.83 & 187.01 & 57.01 & 2,345 & 714.83 \\
\hline 351638116374301 & $14 \mathrm{~N} / 3 \mathrm{E}-26 \mathrm{~K} 1 \mathrm{~S}$ & $11 / 05 / 2014$ & 2,154 & 656.61 & 190.94 & 58.20 & 2,345 & 714.83 \\
\hline 351638116374301 & $14 \mathrm{~N} / 3 \mathrm{E}-26 \mathrm{~K} 1 \mathrm{~S}$ & $05 / 20 / 2015$ & 2,153 & 656.31 & 191.54 & 58.39 & 2,345 & 714.83 \\
\hline 351638116374301 & $14 \mathrm{~N} / 3 \mathrm{E}-26 \mathrm{~K} 1 \mathrm{~S}$ & $07 / 02 / 2015$ & 2,153 & 656.31 & 191.9 & 58.50 & 2,345 & 714.83 \\
\hline 351638116374301 & $14 \mathrm{~N} / 3 \mathrm{E}-26 \mathrm{~K} 1 \mathrm{~S}$ & $05 / 26 / 2016$ & 2,152 & 656.00 & 193.07 & 58.85 & 2,345 & 714.83 \\
\hline 351638116374301 & $14 \mathrm{~N} / 3 \mathrm{E}-26 \mathrm{~K} 1 \mathrm{~S}$ & 06/13/2017 & 2,150 & 655.39 & 194.62 & 59.33 & 2,345 & 714.83 \\
\hline 351638116374303 & $14 \mathrm{~N} / 3 \mathrm{E}-26 \mathrm{~K} 3 \mathrm{~S}$ & $10 / 31 / 2011$ & 2,160 & 658.44 & 185.26 & 56.47 & 2,345 & 714.83 \\
\hline 351638116374303 & $14 \mathrm{~N} / 3 \mathrm{E}-26 \mathrm{~K} 3 \mathrm{~S}$ & $02 / 13 / 2012$ & 2,159 & 658.14 & 185.64 & 56.59 & 2,345 & 714.83 \\
\hline 351638116374303 & $14 \mathrm{~N} / 3 \mathrm{E}-26 \mathrm{~K} 3 \mathrm{~S}$ & $03 / 27 / 2012$ & 2,159 & 658.14 & 186.17 & 56.75 & 2,345 & 714.83 \\
\hline 351638116374303 & $14 \mathrm{~N} / 3 \mathrm{E}-26 \mathrm{~K} 3 \mathrm{~S}$ & $11 / 03 / 2014$ & 2,155 & 656.92 & 189.92 & 57.89 & 2,345 & 714.83 \\
\hline 351638116374303 & $14 \mathrm{~N} / 3 \mathrm{E}-26 \mathrm{~K} 3 \mathrm{~S}$ & $12 / 04 / 2014$ & 2,155 & 656.92 & 189.97 & 57.91 & 2,345 & 714.83 \\
\hline 351638116374303 & $14 \mathrm{~N} / 3 \mathrm{E}-26 \mathrm{~K} 3 \mathrm{~S}$ & $12 / 04 / 2014$ & - & - & 201.53 & 61.43 & 2,345 & 714.83 \\
\hline 351638116374303 & $14 \mathrm{~N} / 3 \mathrm{E}-26 \mathrm{~K} 3 \mathrm{~S}$ & $05 / 20 / 2015$ & 2,154 & 656.61 & 190.7 & 58.13 & 2,345 & 714.83 \\
\hline 351638116374303 & $14 \mathrm{~N} / 3 \mathrm{E}-26 \mathrm{~K} 3 \mathrm{~S}$ & $07 / 02 / 2015$ & 2,154 & 656.61 & 190.91 & 58.20 & 2,345 & 714.83 \\
\hline 351638116374303 & $14 \mathrm{~N} / 3 \mathrm{E}-26 \mathrm{~K} 3 \mathrm{~S}$ & $05 / 26 / 2016$ & 2,153 & 656.31 & 192.15 & 58.57 & 2,345 & 714.83 \\
\hline 351638116374304 & $14 \mathrm{~N} / 3 \mathrm{E}-26 \mathrm{~K} 4 \mathrm{~S}$ & $02 / 13 / 2012$ & 2,159 & 658.14 & 186.42 & 56.83 & 2,345 & 714.83 \\
\hline 351638116374304 & $14 \mathrm{~N} / 3 \mathrm{E}-26 \mathrm{~K} 4 \mathrm{~S}$ & 03/27/2012 & 2,158 & 657.83 & 186.84 & 56.96 & 2,345 & 714.83 \\
\hline 351638116374304 & $14 \mathrm{~N} / 3 \mathrm{E}-26 \mathrm{~K} 4 \mathrm{~S}$ & $11 / 05 / 2014$ & 2,154 & 656.61 & 190.81 & 58.17 & 2,345 & 714.83 \\
\hline 351638116374304 & $14 \mathrm{~N} / 3 \mathrm{E}-26 \mathrm{~K} 4 \mathrm{~S}$ & $12 / 04 / 2014$ & 2,154 & 656.61 & 190.84 & 58.17 & 2,345 & 714.83 \\
\hline 351638116374304 & $14 \mathrm{~N} / 3 \mathrm{E}-26 \mathrm{~K} 4 \mathrm{~S}$ & $05 / 20 / 2015$ & 2,154 & 656.61 & 191.44 & 58.36 & 2,345 & 714.83 \\
\hline 351638116374304 & $14 \mathrm{~N} / 3 \mathrm{E}-26 \mathrm{~K} 4 \mathrm{~S}$ & $07 / 02 / 2015$ & 2,153 & 656.31 & 191.76 & 58.45 & 2,345 & 714.83 \\
\hline 351638116374304 & $14 \mathrm{~N} / 3 \mathrm{E}-26 \mathrm{~K} 4 \mathrm{~S}$ & $05 / 26 / 2016$ & 2,152 & 656.00 & 192.92 & 58.81 & 2,345 & 714.83 \\
\hline 351638116374304 & $14 \mathrm{~N} / 3 \mathrm{E}-26 \mathrm{~K} 4 \mathrm{~S}$ & 06/13/2017 & 2,151 & 655.70 & 194.48 & 59.28 & 2,345 & 714.83 \\
\hline \multicolumn{9}{|c|}{ Local well ID: BP-2, MW-12 } \\
\hline 351654116393301 & $14 N / 3 E-27 E 1 S$ & $01 / 26 / 1993$ & $2,325.8$ & 708.98 & 75.6 & 23.05 & $2,401.4$ & 732.03 \\
\hline 351654116393301 & 14N/3E-27E1S & 09/24/1993 & $2,321.1$ & 707.55 & 80.29 & 24.48 & $2,401.4$ & 732.03 \\
\hline 351654116393301 & $14 N / 3 E-27 E 1 S$ & 08/15/1994 & 2,325 & 708.74 & 76.38 & 23.28 & $2,401.4$ & 732.03 \\
\hline 351654116393301 & $14 \mathrm{~N} / 3 \mathrm{E}-27 \mathrm{E} 1 \mathrm{~S}$ & 09/08/1994 & $2,324.5$ & 708.59 & 76.88 & 23.44 & $2,401.4$ & 732.03 \\
\hline 351654116393301 & $14 \mathrm{~N} / 3 \mathrm{E}-27 \mathrm{E} 1 \mathrm{~S}$ & $02 / 08 / 1995$ & $2,326.1$ & 709.07 & 75.27 & 22.94 & $2,401.4$ & 732.03 \\
\hline
\end{tabular}


Appendix 2. Water-level data for selected wells in Bicycle Basin, Fort Irwin National Training Center, California, 19552017.-Continued

[State well No.: See Well-Numbering System in text. Abbreviations: ID, identification number; mm/dd/yyyy, month/day/year; NAVD 88, North American Vertical Datum of 1988, —, well is dry]

\begin{tabular}{|c|c|c|c|c|c|c|c|c|}
\hline \multirow{2}{*}{ Site ID } & \multirow{2}{*}{$\begin{array}{c}\text { State } \\
\text { well number }\end{array}$} & \multirow{2}{*}{$\begin{array}{c}\text { Date } \\
\text { (mm/dd/yyyy) }\end{array}$} & \multicolumn{2}{|c|}{$\begin{array}{l}\text { Water-surface altitude, } \\
\text { above NAVD } 88\end{array}$} & \multicolumn{2}{|c|}{$\begin{array}{l}\text { Depth to water, below } \\
\text { land surface }\end{array}$} & \multicolumn{2}{|c|}{$\begin{array}{c}\text { Land-surface altitude, } \\
\text { above NAVD } 88^{1}\end{array}$} \\
\hline & & & (feet) & (meters) & (feet) & (meters) & (feet) & (meters) \\
\hline 351654116393301 & $14 \mathrm{~N} / 3 \mathrm{E}-27 \mathrm{E} 1 \mathrm{~S}$ & 05/04/1995 & $2,327.9$ & 709.62 & 73.5 & 22.41 & $2,401.4$ & 732.03 \\
\hline 351654116393301 & $14 \mathrm{~N} / 3 \mathrm{E}-27 \mathrm{E} 1 \mathrm{~S}$ & 08/09/1995 & $2,327.7$ & 709.56 & 73.68 & 22.46 & $2,401.4$ & 732.03 \\
\hline 351654116393301 & $14 \mathrm{~N} / 3 \mathrm{E}-27 \mathrm{E} 1 \mathrm{~S}$ & $12 / 21 / 2010$ & 一 & - & - & 一 & $2,401.4$ & 732.03 \\
\hline \multicolumn{9}{|c|}{ Local well ID: BP-3, MW-13 } \\
\hline 351656116393101 & $14 \mathrm{~N} / 3 \mathrm{E}-27 \mathrm{E} 2 \mathrm{~S}$ & 01/26/1993 & $2,325.8$ & 708.98 & 75.65 & 23.06 & $2,401.4$ & 732.03 \\
\hline 351656116393101 & $14 \mathrm{~N} / 3 \mathrm{E}-27 \mathrm{E} 2 \mathrm{~S}$ & 09/23/1993 & $2,326.8$ & 709.29 & 74.64 & 22.75 & $2,401.4$ & 732.03 \\
\hline 351656116393101 & $14 \mathrm{~N} / 3 \mathrm{E}-27 \mathrm{E} 2 \mathrm{~S}$ & 05/04/1995 & $2,327.9$ & 709.62 & 73.5 & 22.41 & $2,401.4$ & 732.03 \\
\hline 351656116393101 & $14 N / 3 E-27 E 2 S$ & 08/09/1995 & $2,327.7$ & 709.56 & 73.66 & 22.45 & $2,401.4$ & 732.03 \\
\hline 351656116393101 & $14 \mathrm{~N} / 3 \mathrm{E}-27 \mathrm{E} 2 \mathrm{~S}$ & 11/07/1995 & $2,327.5$ & 709.50 & 73.92 & 22.53 & $2,401.4$ & 732.03 \\
\hline 351656116393101 & $14 \mathrm{~N} / 3 \mathrm{E}-27 \mathrm{E} 2 \mathrm{~S}$ & $01 / 30 / 2002$ & - & - & - & - & $2,401.4$ & 732.03 \\
\hline 351656116393101 & $14 \mathrm{~N} / 3 \mathrm{E}-27 \mathrm{E} 2 \mathrm{~S}$ & $12 / 21 / 2010$ & - & - & - & - & $2,401.4$ & 732.03 \\
\hline \multicolumn{9}{|c|}{ Local well ID: BP-4, MW-14 } \\
\hline 351656116392901 & $14 \mathrm{~N} / 3 \mathrm{E}-27 \mathrm{E} 3 \mathrm{~S}$ & 01/26/1993 & $2,324.9$ & 708.71 & 76.48 & 23.31 & $2,401.4$ & 732.03 \\
\hline 351656116392901 & $14 N / 3 E-27 E 3 S$ & 09/24/1993 & $2,320.4$ & 707.34 & 81 & 24.69 & $2,401.4$ & 732.03 \\
\hline 351656116392901 & $14 \mathrm{~N} / 3 \mathrm{E}-27 \mathrm{E} 3 \mathrm{~S}$ & 08/15/1994 & $2,324.2$ & 708.49 & 77.15 & 23.52 & $2,401.4$ & 732.03 \\
\hline 351656116392901 & $14 \mathrm{~N} / 3 \mathrm{E}-27 \mathrm{E} 3 \mathrm{~S}$ & $01 / 30 / 2002$ & $2,327.8$ & 709.59 & 73.56 & 22.42 & $2,401.4$ & 732.03 \\
\hline 351656116392901 & $14 \mathrm{~N} / 3 \mathrm{E}-27 \mathrm{E} 3 \mathrm{~S}$ & 03/01/2005 & $2,329.2$ & 710.02 & 72.25 & 22.02 & $2,401.4$ & 732.03 \\
\hline 351656116392901 & $14 \mathrm{~N} / 3 \mathrm{E}-27 \mathrm{E} 3 \mathrm{~S}$ & $11 / 06 / 2007$ & $2,330.5$ & 710.41 & 70.95 & 21.63 & $2,401.4$ & 732.03 \\
\hline 351656116392901 & $14 \mathrm{~N} / 3 \mathrm{E}-27 \mathrm{E} 3 \mathrm{~S}$ & $12 / 18 / 2007$ & 2,331 & 710.57 & 70.39 & 21.46 & $2,401.4$ & 732.03 \\
\hline 351656116392901 & $14 \mathrm{~N} / 3 \mathrm{E}-27 \mathrm{E} 3 \mathrm{~S}$ & $02 / 16 / 2008$ & $2,330.2$ & 710.32 & 71.25 & 21.72 & $2,401.4$ & 732.03 \\
\hline 351656116392901 & $14 \mathrm{~N} / 3 \mathrm{E}-27 \mathrm{E} 3 \mathrm{~S}$ & $04 / 30 / 2008$ & $2,329.2$ & 710.02 & 72.25 & 22.02 & $2,401.4$ & 732.03 \\
\hline 351656116392901 & $14 N / 3 E-27 E 3 S$ & 07/29/2008 & $2,328.5$ & 709.80 & 72.9 & 22.22 & $2,401.4$ & 732.03 \\
\hline 351656116392901 & $14 \mathrm{~N} / 3 \mathrm{E}-27 \mathrm{E} 3 \mathrm{~S}$ & $01 / 22 / 2009$ & $2,328.5$ & 709.80 & 72.92 & 22.23 & $2,401.4$ & 732.03 \\
\hline 351656116392901 & $14 N / 3 E-27 E 3 S$ & 01/27/2009 & $2,328.4$ & 709.77 & 73.02 & 22.26 & $2,401.4$ & 732.03 \\
\hline 351656116392901 & $14 \mathrm{~N} / 3 \mathrm{E}-27 \mathrm{E} 3 \mathrm{~S}$ & 04/07/2009 & $2,328.8$ & 709.90 & 72.57 & 22.12 & $2,401.4$ & 732.03 \\
\hline 351656116392901 & $14 N / 3 E-27 E 3 S$ & $06 / 15 / 2010$ & $2,328.1$ & 709.68 & 73.27 & 22.34 & $2,401.4$ & 732.03 \\
\hline 351656116392901 & $14 \mathrm{~N} / 3 \mathrm{E}-27 \mathrm{E} 3 \mathrm{~S}$ & $12 / 14 / 2010$ & $2,328.4$ & 709.77 & 73.01 & 22.26 & $2,401.4$ & 732.03 \\
\hline 351656116392901 & $14 \mathrm{~N} / 3 \mathrm{E}-27 \mathrm{E} 3 \mathrm{~S}$ & $12 / 14 / 2010$ & $2,328.4$ & 709.77 & 72.98 & 22.25 & $2,401.4$ & 732.03 \\
\hline 351656116392901 & $14 \mathrm{~N} / 3 \mathrm{E}-27 \mathrm{E} 3 \mathrm{~S}$ & $10 / 25 / 2011$ & $2,328.1$ & 709.68 & 73.26 & 22.33 & $2,401.4$ & 732.03 \\
\hline
\end{tabular}


Appendix 2. Water-level data for selected wells in Bicycle Basin, Fort Irwin National Training Center, California, 19552017.-Continued

[State well No.: See Well-Numbering System in text. Abbreviations: ID, identification number; mm/dd/yyyy, month/day/year; NAVD 88, North American Vertical Datum of 1988, -, well is dry]

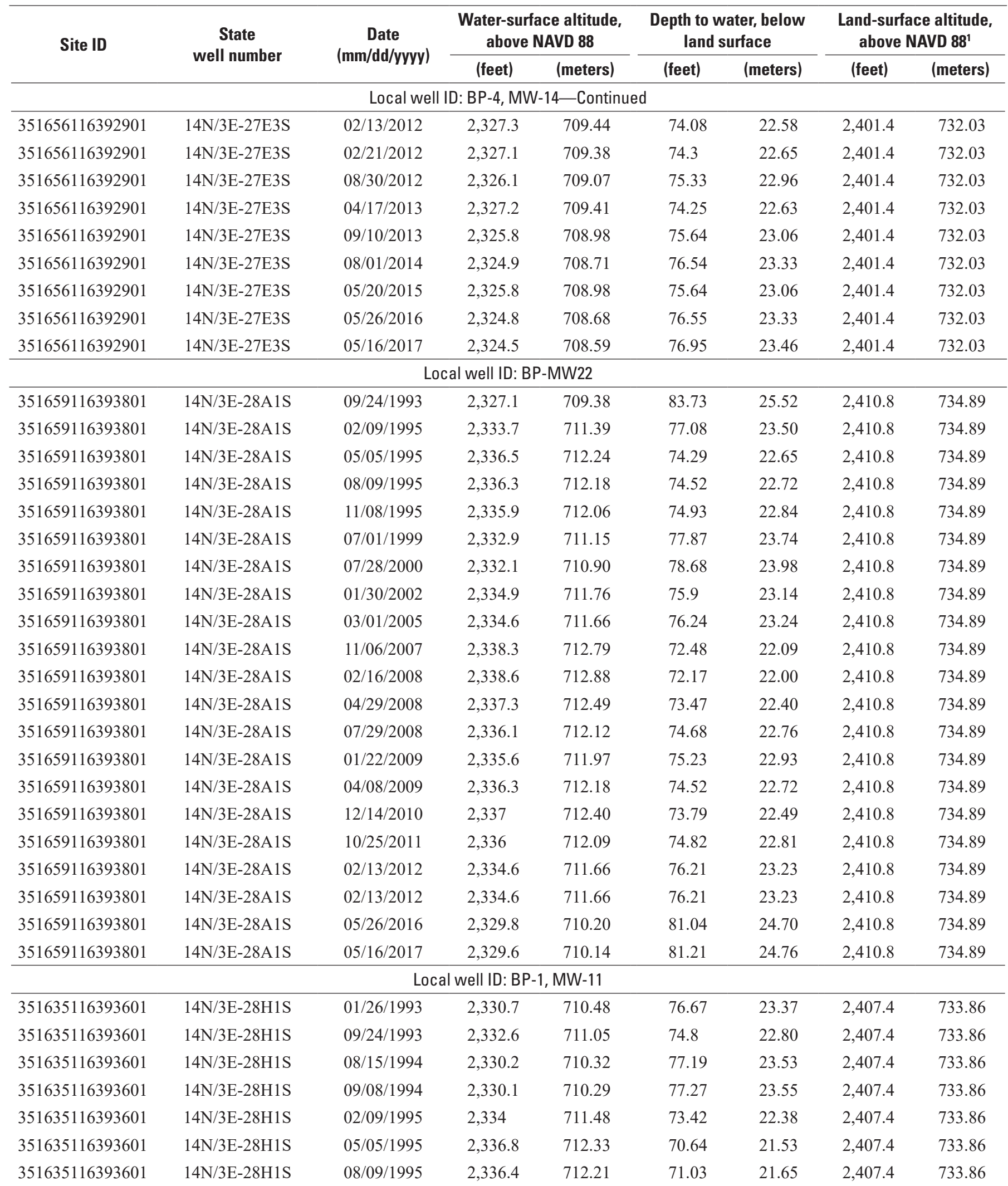


Appendix 2. Water-level data for selected wells in Bicycle Basin, Fort Irwin National Training Center, California, 19552017.-Continued

[State well No.: See Well-Numbering System in text. Abbreviations: ID, identification number; mm/dd/yyyy, month/day/year; NAVD 88, North American Vertical Datum of 1988, - , well is dry]

\begin{tabular}{|c|c|c|c|c|c|c|c|c|}
\hline \multirow[t]{2}{*}{ Site ID } & \multirow{2}{*}{$\begin{array}{c}\text { State } \\
\text { well number }\end{array}$} & \multirow{2}{*}{$\begin{array}{c}\text { Date } \\
\text { (mm/dd/yyyy) }\end{array}$} & \multicolumn{2}{|c|}{$\begin{array}{c}\text { Water-surface altitude, } \\
\text { above NAVD } 88\end{array}$} & \multicolumn{2}{|c|}{$\begin{array}{l}\text { Depth to water, below } \\
\text { land surface }\end{array}$} & \multicolumn{2}{|c|}{$\begin{array}{c}\text { Land-surface altitude, } \\
\text { above NAVD } 88^{1}\end{array}$} \\
\hline & & & (feet) & (meters) & (feet) & (meters) & (feet) & (meters) \\
\hline 351635116393601 & $14 \mathrm{~N} / 3 \mathrm{E}-28 \mathrm{H} 1 \mathrm{~S}$ & $11 / 08 / 1995$ & $2,335.8$ & 712.03 & 71.64 & 21.84 & $2,407.4$ & 733.86 \\
\hline 351635116393601 & $14 \mathrm{~N} / 3 \mathrm{E}-28 \mathrm{H} 1 \mathrm{~S}$ & $01 / 30 / 2002$ & - & & - & - & $2,407.4$ & 733.86 \\
\hline 351656116393401 & $14 \mathrm{~N} / 3 \mathrm{E}-28 \mathrm{H} 2 \mathrm{~S}$ & $09 / 24 / 1993$ & $2,329.1$ & 709.99 & 71.79 & 21.88 & $2,400.9$ & 731.87 \\
\hline 351656116393401 & $14 \mathrm{~N} / 3 \mathrm{E}-28 \mathrm{H} 2 \mathrm{~S}$ & $02 / 09 / 1995$ & $2,330.7$ & 710.48 & 70.21 & 21.40 & $2,400.9$ & 731.87 \\
\hline 351656116393401 & $14 \mathrm{~N} / 3 \mathrm{E}-28 \mathrm{H} 2 \mathrm{~S}$ & $05 / 05 / 1995$ & $2,334.7$ & 711.69 & 66.16 & 20.17 & $2,400.9$ & 731.87 \\
\hline 351656116393401 & $14 \mathrm{~N} / 3 \mathrm{E}-28 \mathrm{H} 2 \mathrm{~S}$ & 08/09/1995 & $2,332.6$ & 711.05 & 68.34 & 20.83 & $2,400.9$ & 731.87 \\
\hline \multicolumn{9}{|c|}{ Local well ID: B-9_Arpt } \\
\hline 351611116380201 & $14 \mathrm{~N} / 3 \mathrm{E}-35 \mathrm{C} 1 \mathrm{~S}$ & $04 / 10 / 1963$ & 2,225 & 678.25 & 127 & 38.71 & 2,352 & 716.97 \\
\hline 351611116380201 & $14 \mathrm{~N} / 3 \mathrm{E}-35 \mathrm{C} 1 \mathrm{~S}$ & $07 / 22 / 1994$ & 2,209 & 710.57 & 143.05 & 6.45 & 2,352 & 716.97 \\
\hline 351611116380201 & $14 \mathrm{~N} / 3 \mathrm{E}-35 \mathrm{C} 1 \mathrm{~S}$ & $07 / 22 / 1994$ & 2,209 & 673.38 & 143.05 & 43.61 & 2,352 & 716.97 \\
\hline 351611116380201 & $14 \mathrm{~N} / 3 \mathrm{E}-35 \mathrm{C} 1 \mathrm{~S}$ & 09/07/1994 & - & - & - & - & 2,352 & 716.97 \\
\hline 351611116380201 & $14 \mathrm{~N} / 3 \mathrm{E}-35 \mathrm{C} 1 \mathrm{~S}$ & $12 / 20 / 1994$ & - & - & - & - & 2,352 & 716.97 \\
\hline 351611116380201 & $14 \mathrm{~N} / 3 \mathrm{E}-35 \mathrm{C} 1 \mathrm{~S}$ & 03/08/1995 & 2,202 & 671.24 & 149.69 & 45.63 & 2,352 & 716.97 \\
\hline 351611116380201 & $14 \mathrm{~N} / 3 \mathrm{E}-35 \mathrm{C} 1 \mathrm{~S}$ & 08/11/1995 & 2,203 & 671.55 & 148.79 & 45.36 & 2,352 & 716.97 \\
\hline 351611116380201 & $14 \mathrm{~N} / 3 \mathrm{E}-35 \mathrm{C} 1 \mathrm{~S}$ & 07/07/1999 & 2,194 & 668.80 & 158.31 & 48.26 & 2,352 & 716.97 \\
\hline 351610116380201 & $14 \mathrm{~N} / 3 \mathrm{E}-35 \mathrm{C} 2 \mathrm{~S}$ & $07 / 28 / 1994$ & $2,207.73$ & 672.99 & 149.67 & 45.62 & $2,357.4$ & 718.61 \\
\hline 351610116380201 & $14 \mathrm{~N} / 3 \mathrm{E}-35 \mathrm{C} 2 \mathrm{~S}$ & 09/08/1994 & $2,207.42$ & 672.90 & 149.98 & 45.72 & $2,357.4$ & 718.61 \\
\hline 351610116380201 & $14 \mathrm{~N} / 3 \mathrm{E}-35 \mathrm{C} 2 \mathrm{~S}$ & $12 / 13 / 1994$ & $2,207.5$ & 672.92 & 149.9 & 45.69 & $2,357.4$ & 718.61 \\
\hline 351610116380201 & $14 \mathrm{~N} / 3 \mathrm{E}-35 \mathrm{C} 2 \mathrm{~S}$ & 03/04/1995 & $2,207.27$ & 672.85 & 150.13 & 45.76 & $2,357.4$ & 718.61 \\
\hline 351610116380201 & $14 \mathrm{~N} / 3 \mathrm{E}-35 \mathrm{C} 2 \mathrm{~S}$ & 08/11/1995 & 2,205 & 672.16 & 152.4 & 46.46 & $2,357.4$ & 718.61 \\
\hline 351610116380201 & $14 \mathrm{~N} / 3 \mathrm{E}-35 \mathrm{C} 2 \mathrm{~S}$ & 07/07/1999 & $2,199.34$ & 670.43 & 158.06 & 48.18 & $2,357.4$ & 718.61 \\
\hline 351610116380201 & $14 \mathrm{~N} / 3 \mathrm{E}-35 \mathrm{C} 2 \mathrm{~S}$ & $03 / 30 / 2000$ & $2,197.72$ & 669.94 & 159.68 & 48.68 & $2,357.4$ & 718.61 \\
\hline 351610116380201 & $14 \mathrm{~N} / 3 \mathrm{E}-35 \mathrm{C} 2 \mathrm{~S}$ & $07 / 28 / 2000$ & $2,195.21$ & 669.17 & 162.19 & 49.44 & $2,357.4$ & 718.61 \\
\hline 351610116380201 & $14 \mathrm{~N} / 3 \mathrm{E}-35 \mathrm{C} 2 \mathrm{~S}$ & $01 / 30 / 2002$ & $2,193.01$ & 668.50 & 164.39 & 50.11 & $2,357.4$ & 718.61 \\
\hline 351610116380201 & $14 \mathrm{~N} / 3 \mathrm{E}-35 \mathrm{C} 2 \mathrm{~S}$ & $03 / 01 / 2005$ & $2,183.69$ & 665.66 & 173.71 & 52.95 & $2,357.4$ & 718.61 \\
\hline 351610116380201 & $14 \mathrm{~N} / 3 \mathrm{E}-35 \mathrm{C} 2 \mathrm{~S}$ & $07 / 29 / 2008$ & - & - & - & - & $2,357.4$ & 718.61 \\
\hline 351610116380201 & $14 \mathrm{~N} / 3 \mathrm{E}-35 \mathrm{C} 2 \mathrm{~S}$ & $01 / 22 / 2009$ & - & - & - & - & $2,357.4$ & 718.61 \\
\hline 351610116380201 & $14 \mathrm{~N} / 3 \mathrm{E}-35 \mathrm{C} 2 \mathrm{~S}$ & $04 / 08 / 2009$ & - & - & - & - & $2,357.4$ & 718.61 \\
\hline 351610116380201 & $14 N / 3 E-35 C 2 S$ & $10 / 09 / 2009$ & - & - & - & - & $2,357.4$ & 718.61 \\
\hline
\end{tabular}


Appendix 2. Water-level data for selected wells in Bicycle Basin, Fort Irwin National Training Center, California, 19552017.-Continued

[State well No.: See Well-Numbering System in text. Abbreviations: ID, identification number; mm/dd/yyyy, month/day/year; NAVD 88, North American Vertical Datum of 1988, , well is dry]

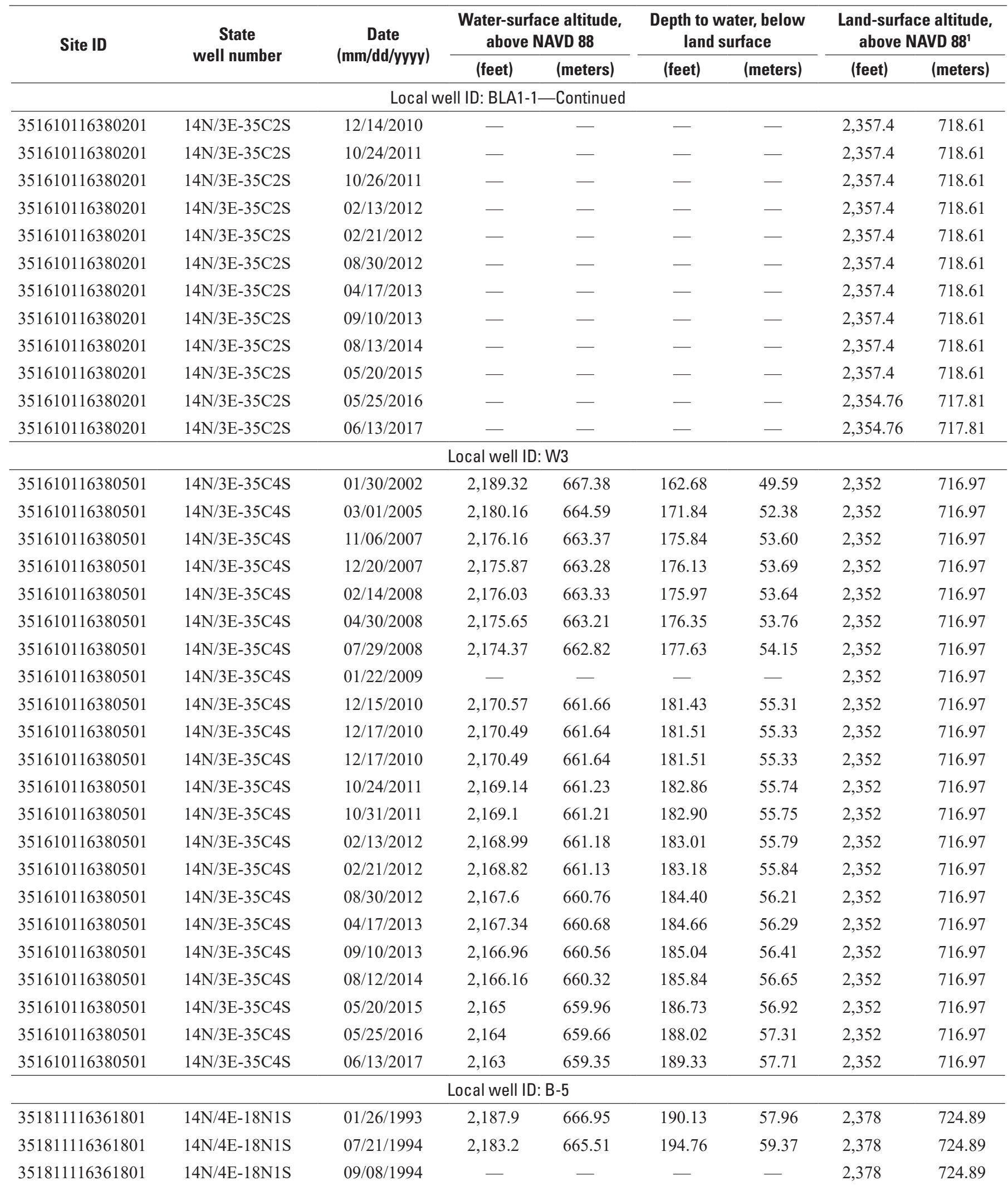


Appendix 2. Water-level data for selected wells in Bicycle Basin, Fort Irwin National Training Center, California, 19552017.-Continued

[State well No.: See Well-Numbering System in text. Abbreviations: ID, identification number; mm/dd/yyyy, month/day/year; NAVD 88, North American Vertical Datum of 1988, - , well is dry]

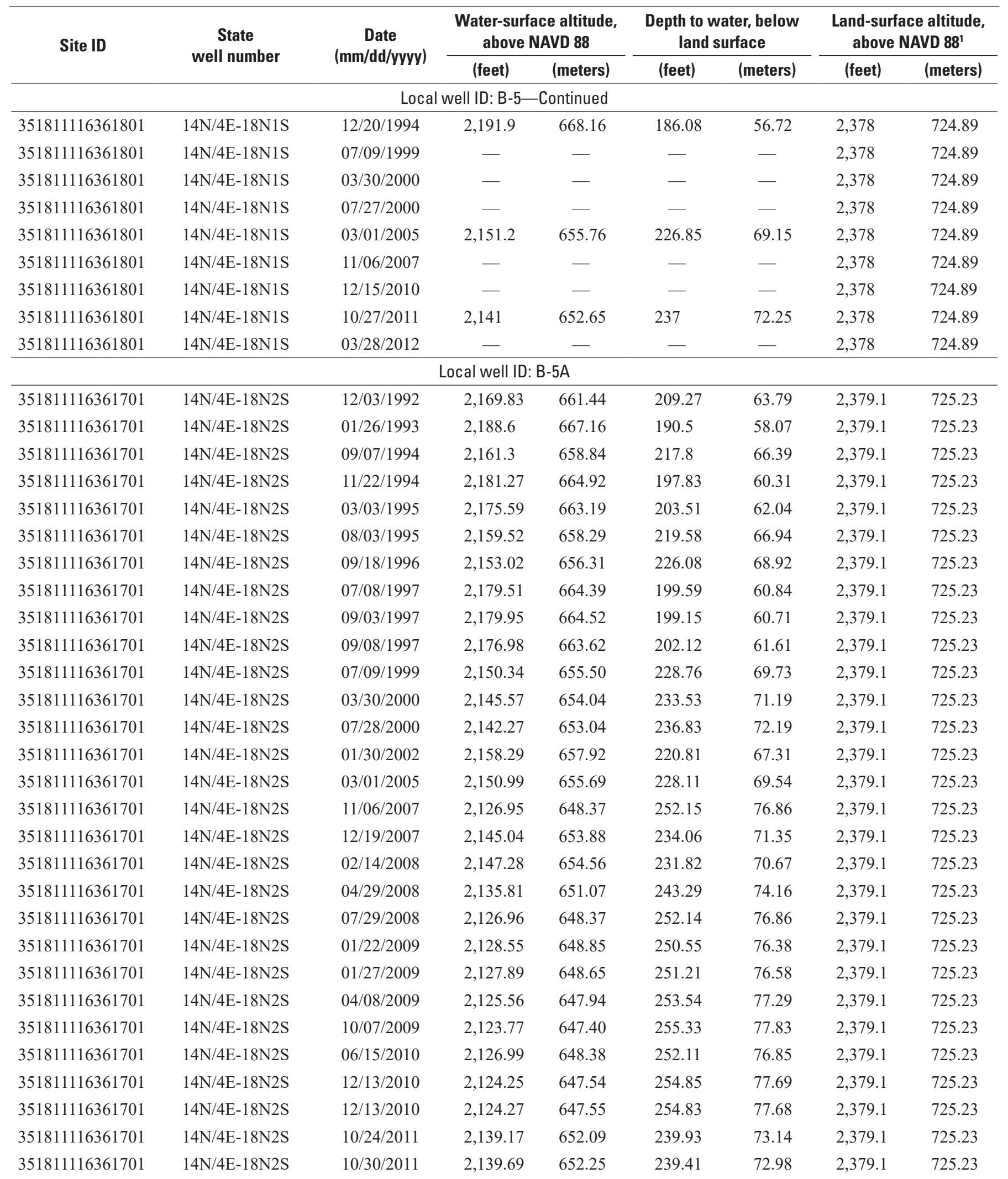


Appendix 2. Water-level data for selected wells in Bicycle Basin, Fort Irwin National Training Center, California, 19552017.-Continued

[State well No.: See Well-Numbering System in text. Abbreviations: ID, identification number; mm/dd/yyyy, month/day/year; NAVD 88, North American Vertical Datum of 1988, , well is dry]

\begin{tabular}{|c|c|c|c|c|c|c|c|c|}
\hline \multirow[t]{2}{*}{ Site ID } & \multirow{2}{*}{$\begin{array}{c}\text { State } \\
\text { well number }\end{array}$} & \multirow{2}{*}{$\begin{array}{c}\text { Date } \\
\text { (mm/dd/yyyy) }\end{array}$} & \multicolumn{2}{|c|}{$\begin{array}{c}\text { Water-surface altitude, } \\
\text { above NAVD } 88\end{array}$} & \multicolumn{2}{|c|}{$\begin{array}{c}\text { Depth to water, below } \\
\text { land surface }\end{array}$} & \multicolumn{2}{|c|}{$\begin{array}{c}\text { Land-surface altitude, } \\
\text { above NAVD } 88^{1}\end{array}$} \\
\hline & & & (feet) & (meters) & (feet) & (meters) & (feet) & (meters) \\
\hline 351811116361701 & $14 \mathrm{~N} / 4 \mathrm{E}-18 \mathrm{~N} 2 \mathrm{~S}$ & $02 / 13 / 2012$ & $2,126.13$ & 648.12 & 252.97 & 77.11 & $2,379.1$ & 725.23 \\
\hline 351811116361701 & $14 \mathrm{~N} / 4 \mathrm{E}-18 \mathrm{~N} 2 \mathrm{~S}$ & $02 / 21 / 2012$ & $2,138.07$ & 651.76 & 241.03 & 73.47 & $2,379.1$ & 725.23 \\
\hline 351811116361701 & $14 \mathrm{~N} / 4 \mathrm{E}-18 \mathrm{~N} 2 \mathrm{~S}$ & 09/10/2013 & $2,143.9$ & 653.53 & 235.2 & 71.70 & $2,379.1$ & 725.23 \\
\hline 351811116361701 & $14 \mathrm{~N} / 4 \mathrm{E}-18 \mathrm{~N} 2 \mathrm{~S}$ & 08/01/2014 & $2,119.73$ & 646.16 & 259.37 & 79.06 & $2,379.1$ & 725.23 \\
\hline 351811116361701 & $14 \mathrm{~N} / 4 \mathrm{E}-18 \mathrm{~N} 2 \mathrm{~S}$ & $02 / 16 / 2015$ & $2,122.77$ & 647.09 & 256.33 & 78.14 & $2,379.1$ & 725.23 \\
\hline 351811116361701 & $14 \mathrm{~N} / 4 \mathrm{E}-18 \mathrm{~N} 2 \mathrm{~S}$ & $05 / 21 / 2015$ & $2,138.84$ & 651.99 & 240.26 & 73.24 & $2,379.1$ & 725.23 \\
\hline 351811116361701 & $14 \mathrm{~N} / 4 \mathrm{E}-18 \mathrm{~N} 2 \mathrm{~S}$ & $04 / 05 / 2016$ & $2,123.1$ & 647.19 & 256 & 78.04 & $2,379.1$ & 725.23 \\
\hline 351811116361701 & $14 \mathrm{~N} / 4 \mathrm{E}-18 \mathrm{~N} 2 \mathrm{~S}$ & $05 / 26 / 2016$ & $2,119.97$ & 646.24 & 259.13 & 78.99 & $2,379.1$ & 725.23 \\
\hline 351811116361701 & $14 \mathrm{~N} / 4 \mathrm{E}-18 \mathrm{~N} 2 \mathrm{~S}$ & $06 / 06 / 2016$ & $2,132.26$ & 649.98 & 246.84 & 75.25 & $2,379.1$ & 725.23 \\
\hline 351811116361701 & $14 \mathrm{~N} / 4 \mathrm{E}-18 \mathrm{~N} 2 \mathrm{~S}$ & 03/08/2017 & $2,137.99$ & 651.73 & 241.11 & 73.50 & $2,379.1$ & 725.23 \\
\hline 351811116361701 & $14 \mathrm{~N} / 4 \mathrm{E}-18 \mathrm{~N} 2 \mathrm{~S}$ & 03/17/2017 & $2,138.21$ & 651.80 & 240.89 & 73.43 & $2,379.1$ & 725.23 \\
\hline 351811116361701 & $14 \mathrm{~N} / 4 \mathrm{E}-18 \mathrm{~N} 2 \mathrm{~S}$ & $03 / 17 / 2017$ & $2,138.29$ & 651.82 & 240.81 & 73.41 & $2,379.1$ & 725.23 \\
\hline 351811116361701 & $14 \mathrm{~N} / 4 \mathrm{E}-18 \mathrm{~N} 2 \mathrm{~S}$ & $05 / 16 / 2017$ & $2,138.88$ & 652.00 & 240.22 & 73.23 & $2,379.1$ & 725.23 \\
\hline
\end{tabular}

${ }^{1}$ Land-surface altitudes in our database may be different because of the results of more exact land surveys completed after publishing this report. 

For more information concerning the research in this report, contact the Director, California Water Science Center U.S. Geological Survey

6000 J Street, Placer Hall

Sacramento, California 95819

https://ca.water.usgs.gov

Publishing support provided by the U.S. Geological Survey Science Publishing Network, Sacramento Publishing Service Center 

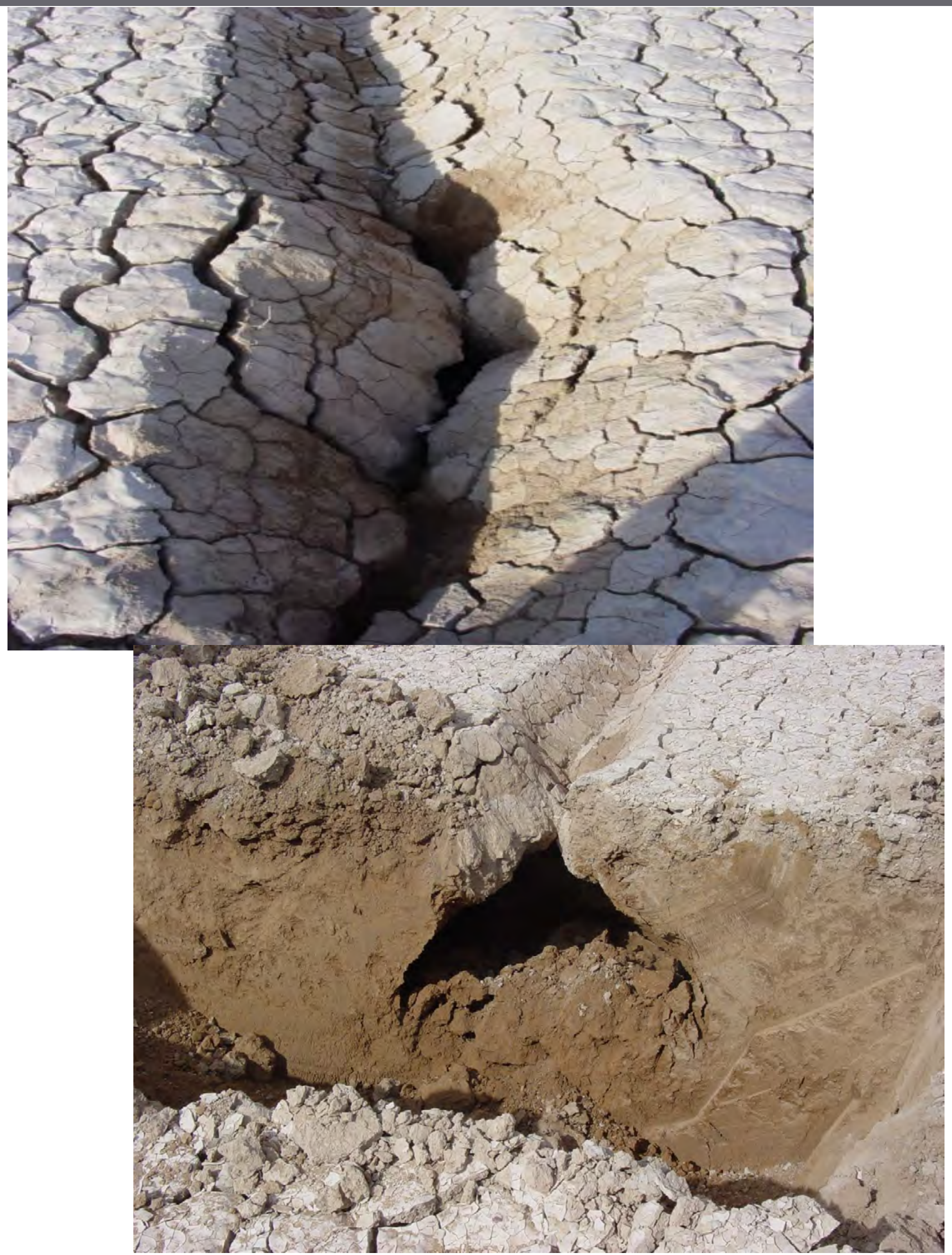